WAOENG- $-91-10$

DE92 000585

\title{
Washington State Biomass Data Book
}

Prepared by:

Joyce A. Deshaye

Energy Specialist

James D. Kerstetter

Bioenergy Program Manager

July 1991

WAOENG 91-10

\section{MASTER}

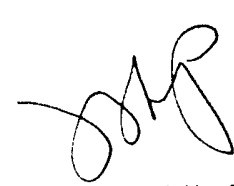

OISTRIBUTION OF THIS DOCUMENT IS UNLIMITED

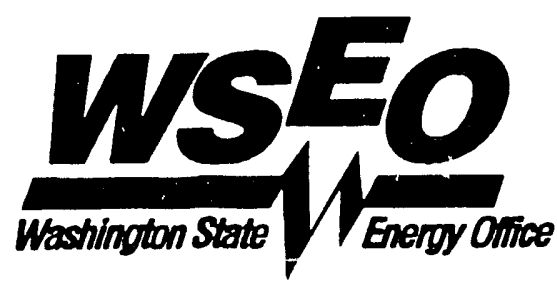




\section{Acknowledgements}

The author wishes to thank the U.S. Department of Energy, Pacific Northwest and Alaska Regional Bioenergy Program, managed by the Bonneville Power Administration, for their financial contribution to this effort.

Thanks are also due Washington State Energy Office (WSEO) staff. Laura Tranin, technical publications manager, provided editorial assistance; Kevin Kozak, cartographer, prepared the maps; and Kristi Kaech, graphic designer, performed the desktop publishing.

\section{Disclaimer}

This report was prepared by the Washington State Energy Office (WSEO) as an account of work sponsored by the United States Department of Energy. Neither the United States, the state of Washington, the Washington State Energy Office, nor any of their employees, nor their contractors, subcontractors, nor their employees, make any warranty, expressed or implied, or assume any legal responsibility for the accuracy, completeness, or usefulness of any information, apparatus, product, or process disclosed within this report. 


\section{Using This Book}

Who Will Benefit?

\section{What Does It Contain?}

This is the first edition of the Washington State Biomass Data Book. It was developed to be a resource for people who work with or are interested in biomass-to-energy projects in Washington. Energy planners and policy makers are encouraged to use the Washington State Biomass Data Book to gain a better understanding of the supply, curreni use, and possible use of biomass fuels.

This one document assesses sources and approximate costs of biomass fuels, presents a view of current users, identifies potential users in the public and private sectors, and lists prices of competing energy resources.

The Summary describes key data from the categories listed above.

Part I, Biomass Supply, presents data in increasing levels of detail on agricultural residues, biogas, municipal solid waste, and wood waste. The information includes type of resource, historical perspective (when available), variability in the quantity of biomass generated, its availability for energy consumption, and costs associated with collection and transportation. Some data are presented on costs of biomass fuels. Further information on agricultural residue pricing and logging residue pricing can be found in Biomass Energy Project Development Guidebook (U.S. Department of Energy/Bonneville Power, 1989); Agricultural Residues (Roy Taylor, 1987); and Regional Logging Residue Supply Curve Project, Vol. 1, Final Report, (U.S. Department of Energy and Bonneville Power Administration, 1986).

Part II, Current Industrial and Commercial Use, demonstrates how biomass is successfully being used in existing facilities as an alternative fuel source. Biomass annually contributes 64 trillion Btu and has a potential of contributing nearly 143 trillion (See Tables S-1 and S-2, page viii). (Washington State Energy Office, 1990). To put this into perspective, Washington's industrial sector consumes nearly 247 trillion Btu of fuels per year. Residential use is estimated to be 154 trillion Btu per year.

Part III, Potential Demand, describes potential energy-intensive public and private sector facilities. Biomass fuels offer a unique energy resource. They are produced on a continuous basis as a result of foud, fiber, and materials needs. Using biomass to fuel the energy needs of these facilities could be one solution to reducing consumption of fossil fuels.

Part IV, Prices of Competing Energy Resources, shows current suppliers of electricity and natural gas and compares utility company rates. This section also includes coal and petroleum 
consumption and pricing patterns in Washington State from 1960 to 1988.

The Bibliography lists the sources used to compile this document. References appear at the end of each section.

Appendix A contains a glossary with brief definitions of words and technical expressions used in this document.

Appendix B contains one sample of the 39 county profiles that have been prepared. Data shown for Spokane County are representative of biomass information available on a per county basis. Each profile gives a detailed look at biomass for a specific county. Information provided includes: climate, population, major cities, principal economic activities, highways, railroads, airports, power generating facilities, utilities, agricultural extension contacts, farms and cropland, landfills, colleges, state facilities, hospitals, schools, etc.. To keep printing costs down, only one county profile has been included in this document. Copies of the other county profiles can be obtained from the Washington State Energy Office.

\section{Sources}

Taylor, Roy. Agricultural Residues. July 1987.

U.S. Department of Energy and the Bonneville Power Administration. Biomass Energy Project Development Guidebook. Prepared for the Pacific Northwest and Alaska Bioenergy Program. 61195-1. 1989.

U.S. Department of Energy and the Bonneville Power Administration. Regional Logging Residue Curve Project, Volume 1, Final Report. August 1986. 


\section{Summary}

Biomass fuels offer a unique energy resource in Washington state. They are produced on a continuous basis as a result of our food, fiber, and material needs. Biomass fuels have a potential of contributing nearly 143 trillion Btu annually.

Major biomass fuels are agricultural field residues (barley and wheat), logging residues, mill residues, and municipal solid waste. Figure $S-1$ shows the contribution of these fuels to the total energy content of biomass generated. Figure S-2 shows the potential energy contribution of biomass assumed to be available for energy.

Although agriculture generates the largest percentage of residues, the majority must remain on the field for agronomic reasons. It is assumed that all logging residues generated are potentially available for energy. Mills generate a considerable amount of residue, but only a small portion goes unused. A portion of all municipal solid waste generated is recycled. Of the amount sent to landfills, only a portion is combustible and considered a fuel. As a result, the combustible portion generated is assumed to be the amount available for energy. Approximately 77 percent of municipal solid waste disposed is combustible and can be used for energy. Secondary wood processing facilities and sewage treatment plants each generate less than 1 percent of the total energy equivalent.

Currently, biomass contributes 64 trillion Btu per year to the industrial and commercial sectors (Washington State Energy Office, 1990).

\section{Availability of Biomass Fuels}

The amount of fuels available at a competitive price is a critical question. Tables S-1 and S-2, page vii, compare the difference between the total amount of biomass generated and the potential amount available for energy. The cost of collecting logging residues and agricultural field residues is currently paid by the energy user. Mill residues and municipal solid waste are often available for free. In some cases the user is paid to haul the material away. Transportation costs are often a major part of the price paid.

1. Of the 8 million tons of barley and wheat field residues generated each year, 1.5 million (equivalent to 22.6 trillion Btu) are available for energy production. Nearly 81 percent of residues generated should be left on the ground for agronomic reasons.

2. Mills generate over 5.1 million dry tons of residues each year. Of that, 182.6 thousand tons are currently available for energy.

3. Nearly 4.2 million dry tons of logging residues equivalent to 75.4 trillion Btu are generated each year from logging 


\section{Biomass Generated}

\begin{tabular}{lrr}
\hline Type & $\begin{array}{r}\text { Biomass } \\
\text { Generated } \\
\text { (Dry Tons) }\end{array}$ & $\begin{array}{r}\text { Energy } \\
\text { Equivalent } \\
\text { (MBtu) }\end{array}$ \\
\hline Agriculture & $7,977,901$ & $119,668,519$ \\
Mill Residues & $5,141,690$ & $92,550,416$ \\
Logging & $4,190,605$ & $75,430,886$ \\
MSW* & $3,123,072$ & $40,599,936$ \\
Sec. Wood Proc. Fac. & 169,899 & $3,058,183$ \\
Biogas & NA & 810,300 \\
Total & $20,603,167$ & $332,118,239$
\end{tabular}

- Figure represents only the combustible portion of disposed municipal solid waste and not the total generated.

Table S-2

Biomass Potentially Available for Energy

\begin{tabular}{lrr}
\hline Type & $\begin{array}{r}\text { Potential } \\
\text { Avallable } \\
\text { (Dry Tons) }\end{array}$ & $\begin{array}{r}\text { Energy } \\
\text { Equivalent } \\
\text { (MBtu) }\end{array}$ \\
\hline Logging & $4,190,605$ & $75,430,886$ \\
MSW* & $3,123,072$ & $40,599,936$ \\
Agriculture & $1,506,311$ & $22,594,666$ \\
Mill Residues & 182,591 & $3,286,629$ \\
Sec. Wood Proc. Fac. & 58,231 & $1,048,159$ \\
Biogas & $N A$ & 6,000 \\
Total & $9,060,810$ & $142,966,276$
\end{tabular}

" Figure represents only the combustible portion of disposed municipal solid waste and not the total generated. 
Figure S-1

Percent of Total Energy Contained in Biomass Generated

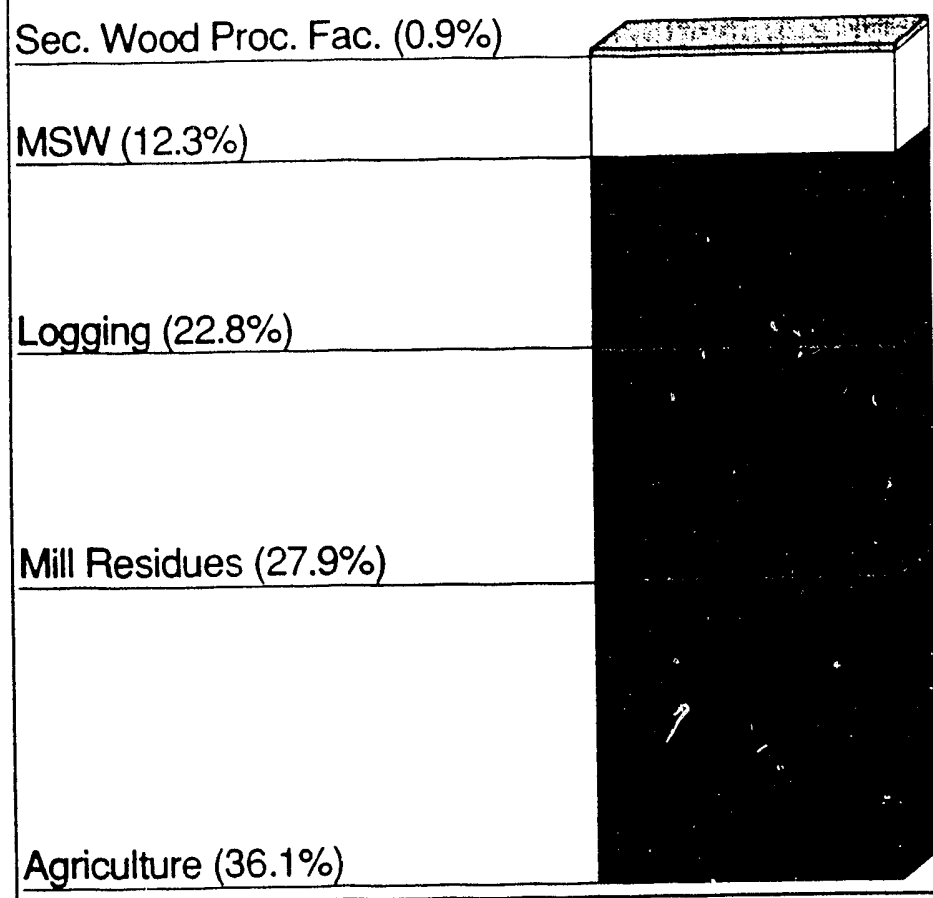

Figure S-2

\section{Percent of Total Energy Contained in Biomass Potentially Available}

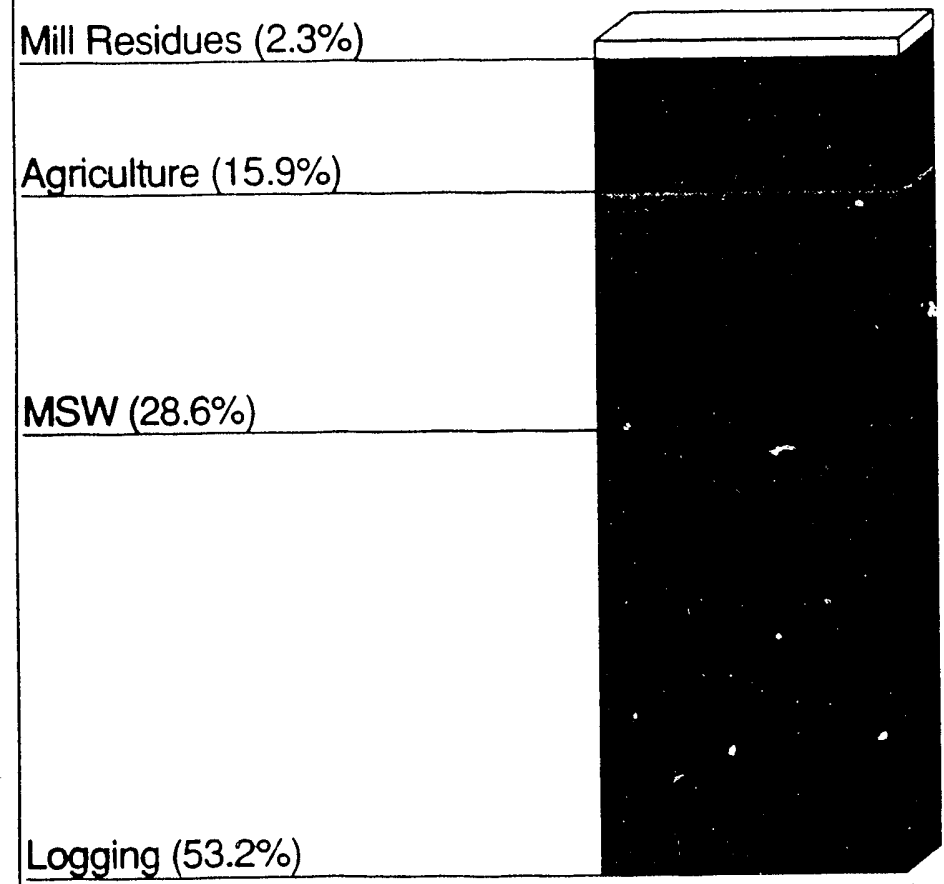




\section{Location of Biomass Fuels}

activities in Washington forests. Over half of these residues are from private lands. Little is known as to how much of that is taken and consumed for energy. It is assumed that most of what is generated is available for energy.

4. Approximately 5.2 million tons of municipal solid wastes are generated in Washington state each year. Over 4 miliion tons of that are disposed and about 1.2 million tons are recycled. Of the amount disposed, a potential of 3.1 million tons ( 77 percent), or 40.6 trillion Btu, can be consumed for energy.

5. Close to 170 thousand tons of residues are generated by secondary wood processing facilities in Washington state. Over 58 thousand tons, slightly over 1 trillion Btu equivalent, can potentially be used for energy.

6. Sewage treatment facilities generate 1.3 billion cubic feet of biogas each year. Currently, approximately 10 million cubic feet, equivalent to 6 billion Btu, are available for energy. Biogas is used for space and process heat requirements of sewage treatment plants. Some larger facilities clean and scrub biogas to pipeline quality and sell it to utilities.

Almost all biomass fueis have one common characteristic: they are produced as a residue from some other primary process. Logging and wood product residues are generated because of our need for fiber and structural building materials; agricultural residues result from our need for food or fiber; and biogas and municipal solid waste come from our consumer habits. Whatever the primary purpose, biomass fuels will be used close to their point of generation.

1. For the most part, barley, spring wheat, and winter wheat field residues can be found on farms in eastern Washington. The southeast region generates approximately 58 percent of the total for both gross and net residues. Winter wheat dominates, generating 90 percent of the total for the state.

2. Mill residues are generated from sawmills, veneer and plywood mills, and shake and shingle mills. About 79 percent of available residues are from the shake and shingle mills found primarily in western Washington.

3. Logging residues on private forest lands represent 56 percent of the state total. On average, nearly 2.4 million tons of residues are generated annually. The southwest region dominates.

4. Since municipal solid waste and biogas generation is directly related to human activities, the largest population bases in Washington generate the greatest amount of wastes. The Puget Sound region in western Washington ranks number one in both categories. 


\section{A View of Current Users}

\section{Potential Users in the Public and Private Sectors}

Historically, the wood products industries and the residential sectors in Washington state have used wood residues to meet a significant portion of their fuel needs. Agricultural residues, biogas, and municipal solid waste have only recently been valued for their energy potential.

1. At this time, little if any data are available on agricultural residues used for energy.

2. Data on logging residues taken from forests are hard to document. Currently, it is not known how much is being taken from forests and used for energy consumption. The residential sector consumes most of the residues removed for energy.

3. Mill residues are being used for pulp and board, fuel, and for other purposes. Only a small percent of these residues go unused.

4. There are 59 wood combustion facilities generating 59 trillion Btu per year. Forest products industries often use residues in-house to produce energy for space and process heat. Some is sold to utility companies.

5. Currently, there are three municipal solid waste combustion facilities in Washington state, a fourth is under construction and is expected to be operational in 1992. They generate over 54 megawatts of electricity.

6. There are 39 sewage treatment plants that generate, on avera $b c$. 810 billion Btu per year. Smaller plants use biogas in-house for space and process heat. Although larger plants use biogas for the same purposes, some scrub it and sell it to natural gas utilities or generate and sell electricity.

7. Secondary wood processors are generally smaller businesses and usually give residues away. These residues are used for soil improvement, animal bedding, and some are used as fuel. An accurate count of secondary wood processors is difficult to obtain and is not given in this report.

Energy-intensive facilities in Washington state, such as state buildings, schools, hospitals, and industrial facilities could benefit from using biomass fuels.

1. The industrial sector consumes a total of 247 trillion Btu of energy per year. It follows the transportation sector, which consumes 527 trillion Btu. Approximately 255 industrial facilities were identified from this sector as potential biomass users. 


\section{Competing Energy Resources}

\section{Sources}

2. The commercial sector consumes a total of 116 trillion Btu of energy each year. There are approximately 128 state agencies, 39 colleges and universities, $500 \mathrm{~K}-12$ private and public schools, and 111 hospitals in this state whose energy consumption patterns can be analyzed to determine the feasibility of biomass fuel use.

As Washington state's population grows, so does energy demand. Because biomass fuels are produced on a continuous basis they offer a unique energy resource. More widely used energy resources are hydropower, petroleum, coal, and natural gas. In order to compete, biomass must be available at prices competitive with these resources.

1. Washington state currently relies heavily on hydropower to fuel the electrical energy demands in the state. Washington Water Power's Kettle Falls facility is the only investor owned utility utilizing biomass to generate electricity.

2. In 1991, energy demands increased taking Washington state from an era of electrical energy surplus to projected energy shortfall.

3. In 1986 (last year data available), the residential sector cut residential heating oil consumption by nearly 50 percent from 1960 (first year data available). Residual fuel consumption by the industrial sector remained approximately the same.

4. In 1986, coal consumed by the residential, commercial, and industrial sectors was reduced by approximately 50 percent from 1960. Coal consumed by electric utilities increased by approximately 30 percent from 1972 (first year coal was used to generate electricity).

5. Nearly all natural gas consumed in Washington state is imported from other states.

Washington State Energy Ofice. 1990 Washington State Directory of Biomass Energy Facilities. 1990. 


\section{Contents}

Using This Book

Who Will Benefit

What Does It Contain?

Sources

Summary

xii

Availability of Biomass Fuels

xii

Locationof Biomass Fuels

A View of Current Users

$x$

Potential Users in the Public and Private Sectors

$x i$

Competing Energy Resources

Part L. Biomass Supply

Agricultural Field Residues

I-2

Biogas

Municipal Solid Waste

I-4

Wood Waste

I-4

Forest Residues

I-6

Mill Residues

I-6

Secondary Wood Processing

I-8

Sources

I-11

I-12

Part II. Current Industrial and Commercial Use

II-1

Agricultural Residues

II-1

Biogas Use

II-1

Municipal Solid Waste

II-2

Wood Wastes

II-2

Sources

II-3

Part III. Potential Demand

III-1

State Facilities and Schools

III-1

Hospitals

III-2

Industrial Users

III-2

Sources

III-2

Part IV. Prices of Competing Energy Resources

IV-1

Electric Companies

IV-1

Natural Gas Companies

IV -1

Coal

IV-1

Petroleum

IV-2

Sources

IV-2

Part V. Bibliography

V-1

Appendix A. Glossary

A-1

Appendix B. Spokane County Profile

B-1 


\section{Tables}

\section{Biomass}

S-1 Biomass Generated

S-2 Biomass Energy Potentially Available for Energy

viii

I-1 Future Availability and Price of Biomass Fuels

viii

I-2 Factors Determining the Cost of Residues

I-3 Transportation Costs - UTC Rates

Agricultural Residues

I-4 Gross Barley, Spring Wheat, Winter Wheat Residues by Region

I-5 Net Barley, Spring Wheat, Winter Wheat Residues by Region

I-6 Gross Barley Residues by Region

I-7 Net Barley Residues by Region

I-8 Gross Spring Wheat Residues by Region

I-9 Net Spring Wheat Residues by Region

I-10 Gross Winter Wheat Residues by Region

I-11 Net Winter Wheat Residues by Region

I-12 Gross Barley, Spring Wheat, Winter Wheat Residues by County

I-13 Net Barley, Spring Wheat, Winter Wheat Residues by County

I-14 Gross Barley Residue ty County

I-15 Net Barley Residues oy County

I-16 Gross Spring Wheat Kesidues by County

I-17 Net Spring Wheat Residues by County

I-18 Gross Winter Wheat Residues by County

I-19 Net Winter Wheat Residues by County

\section{Biogas}

I-21 1989 Biogas Generated by Region 


\section{Forest Residues}

I-25 Logging Residues on All Forest Land Ownerships by Region

I-26 Logging Residues on Private Forest Lands by Region I-52

I-27 Logging Residues on National Forest Lands by Region

I-28 Logging Residues on State Forest Lands by Region

I-29 Logging Residues on Indian Forest Lands by Region

I-30 Logging Residues on Other Federal and Non-Federal Forest Lands by Region

I-31 Logging Residues on All Forest Land Ownerships by County

I-32 Logging Residues on Private Forest Lands by County

I-33 Logging Residues on National Forest Lands by County I-57

I-34 Logging Residues on State Forest Lands by County I-58

I-35 Logging Residues on Indian Forest Lands by County

I-36 Logging Residues on Other Federal and Non-Federal Forest Lands by County

I-37 Logging Residues Generated and Amount Available at a Marginal Cost of less than \$3.30/MBTU

\section{Mill Residues}

I-38 Generated Mill Residues by Region

1-39 Unused Mill Residues by Region

I-40 Generated Sawmill Residues by Region

I-41 Unused Sawmill Residues by Region

I-42 Generated Shake \& Shingle Mill Residues by Region

I-43 Unused Shake \& Shingle Mill Residues by Region

I-44 Generated Veneer \& Plywood Mill Residues by Region

I-45 Unused Veneer \& Plywood Mill Residues by Region

I-46 Mill Residues Generated and Amount Available for Cost of Transportation 
Secondary Wood Processing Facilities Residues

I-47 Generated Secondary Wood Processing

Facilities Residues by Region

I-48 Unused Secondary Wood Processing

Facilities Residues by Region

I-49 Generated Secondary Wood Processing Facilities Residues by County

I-50 Unused Secondary Wood Processing Facilities Residues by County

Municipal Solid Waste

I-23 1987 Combustible Fraction of Municipal Solid Waste

I-24 Estimated Waste Generated In 1989 by County

Current Biomass Use

II-1 Biogas Production Facilities

II-4

II-2 Municipal Solid Waste Combustion Facilities

II-3 Wood Combustion Facilities- Forest Products Industries

II-6

II-4 Wood Combustion Facilities - State

II-5 Wood Combustion Facilities - Utility Companies

II-6 Solid Fuel Processing Facilities

\section{Potential Biomass Use}

III-1 Washington State Total Energy Consump: inn by Sector

III-3

III-2 Average Square Feet of State Buildings by County

III-4

III-3 Average Square Feet of School Buildings by County

III-5

III-4 Washington State Hospitals

III-6

III-5 Average Number of Hospital Beds Per Region

III-9

III-6 Average Number of Hospital Beds by County

III-10

III-7 Fuel Consumed by Potential Industrial Biomass Users by County

\section{Electric Utilities}

IV-1 Washington State Electric Utilities Rates per Kilowatt Hour 
IV-2 Washington State Electric Utility Rates per Kilowatt Hour by Region

IV-3 Net Generation, Fuel Consumption, and Fuel Stock by Company and Plant

Gas Utilities

IV-4 Natural and Other Gases Produced and Purchased by Natural Gas Pipeline Companies, October 1990

IV-5 Washington State Natural Gas Utility Rates

Coal

IV-6 Washington State Coal Consumption and Prices by Sector

Oil

IV-7 Washington State Residential Heating Oil and Residual Fuel Consumption and Price 


\section{Figures}

\section{Biomass}

S-1 Percent of Total Energy Contained in Biomass Generated

S-2 Percent of Total Energy Contained in Biomass Energy Potential

Agricultural Residues

I-1 Totả Annual Gross \& Net Barley, Spring Wheat \& Winter Wheat Residues 1982 - 1989

I-2 Total Annual Gross \& Net Barley Residues, 1982-1988

I-3 Total Annual Gross \& Net Spring Wheat Residues, $1982-1988$

I-4 Total Annual Gross \& Net W inter Wheat Residues $1982-1988$

Logging Residues

1-5 Percent of Logging Residues Generated by Land Ownership

I-6 Total Annual Logging Residues on All Forest Land Ownerships, 1981 - 1988

I-7 Total Annual Logging Residues on Private Forest Lands, 1981 - 1988

I-8 Total Annual Loggingg Residues on National Forest Lands, 1981 - 1988

I-9 Total Annual Logging Residues on State Forest Lands, 1981 - 1988

I-10 Total Annual Logging Residues on Indian Forest Lands, 1981 - 1988

1-11 Total Annual Logging Residues on Other Federal \& Non-Federal Forest Lands, $1981-1988$

\section{Mill Residues}

I-12 Total Annual Mill Residues Generated and Unused, 1982 - 1988

I-13 Total Annual Sawmill Residues Generated and Unused 1982 - 1988 
I-14 Total Annual Shake and Shingle Mills Residues

Generated and Unused, 1982 - 1988

I-15 Total Annual Veneer \& Plywood Residues Generated and Unused, 1982 - 1988 


\section{Maps}

Agricultural Residues

I-1 Barley, Spring Wheat and Winter Wheat Combined Gross Residues

I-2 Barley, Spring Wheat and Winter Wheat Combined Net Residues

I-3 Gross Barley Residues

I-4 Net Barley Residues

I-5 Gross Spring Wheat Residues

I-6 Net Spring Wieat Residues

I-7 Gross Winter Wheat Residues

I-8 Net Winter Wheat Residues

\section{Biogas}

I-9 Biogas Output

Forest Residues

I-11 Total Logging Residues

I-12 Logging Residues on Private Lands

I-13 Logging Residues on National Lands

I-14 Logging Residues on State Lands

I-15 Logging Residues on Indian Lands

I-16 Logging Residues on Other Federal \& Non-federal Lands

Mill Residues

I-17 Generated Mill Residues

I-18 Unused Mill Residues

I-19 Generated Sawmill Residues

I-20 Unused Sawmill Residues

I-21 Generated Shake and Shingle Mill Residues

I-22 Unused Shake and Shingle Mill Residues

I-21 Generated Veneer \& Plywood Mill Residues

I-22 Unused Veneer \& Plywood Mill Residues

\section{Municipal Solid Waste}

I-10 1987 Municipal Solid Waste 
Secondary Wood Processing Facilities Residues

I-25 1989 Estimated Generated Wood Residues from Secondary Wood Processing Facilities

I-26 1989 Estimated Unused Wood Residues from Secondary Wood Processing Facilities 


\section{Part I}

\section{Biomass Supply}




\section{Part I}

\section{Biomass Supply}

The economy of Washington has traditionally been based on natural resources. The forest products and agricultural industries are dominant and they both generate large quantities of wastes that could be used for energy. The type and source of biomass fuel varies widely within the state both on a geographical and a time basis. In general, because these fuels have a low density they cannot be economically transported any great distance.

Agricultural field residues, biogas, municipal solid waste, logging residues, mill residues, and secondary wood processing residues are discussed in this section. Averages and ranges are presented by region and by county for agricultural, logging, and mill residues that were generated and available over an 8- to 10-year period for the state. Since data have not been available until recently, biogas, municipal solid waste, and secondary wood processing facility residue averages and ranges are not presented.

The data are presented in increasing levels of detail for each type of biomass. The first level presents an historical perspective and views the state as a whole. The next level defines geographic areas to represent potential supply zones. The final level gives details for each county. Figures, maps, and tables are presented for analysis under each type of biomass.

Also, other significant details, such as mill type and forest ownership, are presented in separate tables. Tables I-1 to I-3 show future availability and estimated prices of biomass fuels, factors determining costs, and transportation costs.

To represent the geographic areas in this book supply zones were borrowed from preestablished "Waste Generation Areas (WGA)" defined by the Washington State Department of Ecology (The Matrix Management Group et. al, 1988). These areas are:

Area

West

Northwest

Puget Sound

Southwest

\section{Counties}

Clallam, Grays Harbor, Jefferson, Mason, Pacific

Island, San Juan, Skagit, Whatcom

King, Kitsap, Pierce, Snohomish, Thurston

Clark, Cowlitz, Lewis, Skamania, Wahkiakum 
North Central

South Central

Northeast

Southeast
Chelan, Douglas, Grant, Okanogan

Kittitas, Klickitat, Yakima

Ferry, Lincoln, Pend Oreille, Spokane, Stevens

Adams, Asotin, Benton, Columbia, Franklin, Garfield, Walla Walla, Whitman

The Department of Ecology used the following geographical factors as part of its criteria for selecting Waste Generation Areas: percapita income, population, total employment in lumber and wood-products industries, total employment in nonlumber manufacturing, percent of area in harvested cropland, number of recycling centers, transportation costs to market for scrap paper, and average annual precipitation (The Matrix Management Group et.al, 1988).

\section{Agricultural Field Residues}

\section{Methodology of Data Collected}

Agricultural field residues are generated each year in Washington state as a result of the demand for food to feed the nation and the world. Demand for food is determined by population and the relative competitive position of the state in supplying needed crops.

Crops are the primary products; residues are what remain on the land after the harvest. Vines and culls from vegetable yields, stubble and straw from small grain and grass crops, stalks and cobs from corn production, and prunings from orchards and vineyards are all residues that can potentially be used for energy. Barley and wheat production predominate in Washington state; therefore, stubble and straw generated from the production of these grains are the only residues considered in this document.

The amount of residues generated each year and the quantity available for energy vary greatly. The quantity generated depends upon the yield of grain produced and the residue factors for that particular type of grain. The quantity available for energy depends upon the agronomic requirements for how much residues should be left on the ground to provide for erosion control and soil fertility.

Barley and wheat residues have a heating value of approximately 15 million British thermal units per dry ton.

Barley and wheat production figures were taken from the annual editions of Washington Agricultural Statistics, published by the Washington State Department of Agriculture, for the years 1982 through 1989. Residue factors of 1.5, 1.3, and 1.7 pounds of straw per pound of grain produced for barley, spring wheat, and winter wheat, respectively, were used.

The minimum amount of residue required to be left on a field for agronomic reasons depends on the individual site and the type of 
farming. Dry land farming requires nore surface mulch to maintain and protect the natural moisture from evaporation; irrigated farming requires less. For the purpose of this document, we made the following assumptions concerning soil improvement requirements: dry land farming requires 2.5 tons of residue per acre be left and irrigated farming requires 2.0 tons per acre be left.

All residues generated as a result of grain harvest were labeled gross residues. Residues available for energy after the percentage left for soil improvement were labeled net residues.

The gross quantity of residue generated was found by multiplying the grain yield data by the residue factor. To calculate net residues, the amount left for soil improvement was subtracted from the gross quantity generated.

Dry and irrigated land were calculated separately for both gross and net residues. (Results for Spokane county are given in Appendix B. Results for other counties are available upon request from the Washington State Energy Office). The values reported in the this section of the document are combined for dry and irrigated lands.

\section{Current Supply}

\section{Future Availability and Cost}

Pages I-17 through I-36 show the current supply of barley, spring wheat, and winter wheat in increasing levels of detail. Figure I-1 shows the total amount of barley and wheat generated (gross) and available for energy (net) over an 8-year period from 1982 through 1989. Figures I-2 through I-4 give the amount per year by type of field residue (see Appendix B for actual figures by county). Tables I-4 through I- 19 represent the average and range available for the state, regionally and by county. The net average for the state was over 1.5 million dry tons, which was 19 percent of the gross. The southeast region dominated, producing 58 percent of the net (see Table I-5). Counties having the greatest amount of residues available for energy were Whitman, Grant, Adams, Lincoln, and Walla Walla - all are located in Eastern Washington. Maps I-1 through I-8 show the gross and net annual average of agricultural field residues in dry tons by region and by type.

Approximately 90 percent (Figure I-5) of the 1.5 million dry tons of the total net residues were winter wheat. Winter wheat averaged 1.35 million dry tons, spring wheat averaged nearly 112 thousand dry tons, and barley averaged 45 thousand dry tons annually. The southeast region dominated for both types of wheat; the north central region dominated for barley. Adams and Grant counties ranked in the top five net generators for barley and wheat.

Barley and wheat residues generated and available for energy over the next two decade are assumed to be similar to the period between 1982 through 1989. The total net average being 1.5 million tons with an estimated range of 1 to 2.6 million tons. Eastern Washington is expected to produce the greatest percentage of agricultural field residues available for energy, with the southeas, region predominating. 


\section{Biogas}

\section{Methodology of Data Collected}

\author{
Current Supply
}

\section{Municipal Solid Waste}

Residue collection costs depend on the amount of residue per acre that can be removed with agronomic limits. The transportation costs can be high because of the low bulk density of the material. The trucks generally do not carry a full weight load and thus the cost per ton is higher than, for instance, logging residues. Table I20 shows the estimated costs for collecting and transporting agricultural residues.

Biogas is the mixture of carbon dioxide and methane produced through the bacterial action of decomposing organic materials found in municipal solid wastes, sewage, and livestock manures. Gas is captured from these wastes through a process called anaerobic digestion. In this process, sludge is placed in a digester for a selected length of time and allowed to decompose. The gas released from this process is called biogas and has a heating value of approximately 600 British thermal units per standard cubic foot. Commercial natural gas, a combination of methane, propane, and butane, has a net heating value of approximately 1,050 Btu.

Although biogas can be generated from municipal solid wastes on landfills and manure on farms, in Washington state it is primarily captured from sewage treatment plants. For this reason, only biogas generated from these facilities is included in this document.

Figures were taken directly from the 1990 Washington State Directory of Biomass Energy Facilities, published by the Washington State Energy Office (WSEO) in 1990.

Currently, Washington state generates approximately 1.4 trillion cubic feet of biogas annually. For the most part, it is produced at sewage plants from human waste. Pages I-37 through I-40 shows the amount of biogas generated in increasing levels of detail. The Puget Sound region generates the greatest percentage- 71 percent of the total at 956 million cubic feet per year $(573,600 \mathrm{M} \mathrm{Btu})$, see Table I-21 and Map 1-9. The most densely populated counties are the highest generators: King, Spokane, Pierce, Kitsap, and Thurston counties, see Table I-22.

Municipal solid waste (MSW) is generated by activities from the residential, industrial, manufacturing, construction, and governmental sectors of our economy. The quantity generated can be calculated based on the population and residue factor. It consists of newspaper, mixed waste paper, corrugated cardboard, glass, aluminum, tin cans, other metals, chemicals, textiles, leather, appliances, plastic, polystyrene, tires, other rubber products, wood waste, disposable diapers, oil, batteries, hazardous waste, constiur tion and demolition debris, and other organic and inorganic wa tes.

A certain percentage of the total waste generated is diverted from the landfills through recycling programs. The rest is either landfilled or incinerated. 


\section{Methodology of Data Collection}

\section{Current Supply}

Future Availability and Cost
The figures in this document were based on the combustible fraction of disposed wastes and not from the total generated. Combustible wastes were defined as newsprint, corrugated containers, computer paper, office paper, mixed waste paper, nonrecyclable paper, PET bottles, HDPE bottles, other plastic packaging, expanded polystyrene, rubber products, tires, food, lawn and garden waste, wood waste, disposable diapers, textiles, and leather not currently recycled.

The figures given are based on the Best Management Practices Analysis for Solid Waste, 1987 Recycling and Waste Stream Survey, Volume 1, prepared by The Matrix Management Group, R.W. Beck and Associates and the Gilmore Research Group, for the Washington State Department of Ecology in 1988. These figures were for 1987 only. An annual average was not calculated.

Construction and demolition wood wastes are still being landfilled because the variation in their physical and chemical characteristics makes it challenging to find end-use markets. Therefore, construction and demolition wood wastes are included in this section of the document as opposed to the wood waste section.

Since municipal solid wastes are in essence "by-products" resulting from the activities of our society, those areas having the greatest population generate the most waste. Currently, the state of Washington generates over 5 million tons of municipal solid waste annually; approximately 22 percent of that is recycled. (This percentage is on the rise as municipalities are required by law to implement recycling programs in their communities.) Nearly 77 percent of what is currently sent to the landfills could potentially be recovered and burned to provide 39 trillion Btu of refuse derived fuel annually.

Pages I-41 through I-43 show the potential amount of combustible municipal solid waste generated in Washington state. The Puget Sound region has the densest population base and generates close to 60 percent of the total municipal solid waste disposed in the state, see Table I-23 and Map I-10. The top five counties generating the greatest percentages are: King, 35 percent; Pierce, 14 percent; Snohomish, 11 percent; Spokane, 9 percent; and Clark, 5 percent, see Table I-24.

Changing landfill regulations, public interest in recycling, consumer habits, and population growth all have an impact on the availability of municipal solid waste for energy.

As landfill regulations become more stringent the cost of land disposal increases. Thus for the many sectors of our economy, the cost of disposing their solid wastes becomes increasingly more expensive. Some industries cannot afford the disposal costs and are finding alternatives to landfilling, such as paying a lesser fee to users to dispose their wastes, using waste in-house to generate electricity, or recycling. In most cases, it is projected that recycling 


\section{Wood Wastes}

\section{Forest Residues}

programs will keep the level of solid waste requiring disposal from growing significantly over the next 20 years.

Municipal solid waste is a result of consumerism and thus is a function of population and economic activity within a supply zone. The quantity of solid waste that is generated can be calculated based on the population of an area and a residue factor. The Washington State Department of Ecology estimates 6.26 pounds of garbage per person per day.

When all other uses for municipal solid waste have been exhausted, it ends up being disposed in our landfills. As a result, it has zero value and often is available free. Often the user is paid to haul the material away.

The supply of wood wastes is determined by the market for wood and wood products. Forest residues are produced because timber is needed to produce lumber, pulp, and plywood. Mill residues are produced as a result of producing lumber, plywood, shakes, and shingles. Residues produced from secondary wood processing facilities result from manufacturing such items as kitchen cabinets, flooring, and pallets.

Washington has a large wood products industry that uses wood fuels extensively to produce energy for space and process heat. It is one of the leading timber industry states, producing 11 percent of the nation's softwood lumber, 7 percent of the nation's plywood, and 62 percent of the nation's softwood log exports (DNR, 1988).

Forest residue is a general term that encompasses several sources of potential biomass fuels, all of which have a common aspect of being generated in a forest environment. The most common forest residues are called logging residues. Logging residues are a result of commercial logging operations in which trees are cut and merchantable material is removed from the forest. The residues, in general terms, consist of all down and dead woody material existing on an area after timber harvest is completed. Other categories of forest biomass that could be used for energy include stagnant and dying timber, hardwood stand conversions, and precommercial thinnings. The economics of recovery are currently such that only logging residues are considered as available for energy uses. The other categories of material often are in unroaded areas and/or the concentration of residues makes their recovery for fuel uneconomic. Furthermore, the data on resource availability and the cost of recovering these other categories of forest residues are not readily available. This section will therefore only assess the availability of logging residues.

Typically, forest residues are burned on-site or left to decompose in the forest, unless the landowner requires removal of the waste. 


\section{Current Supply}

Residues per acre harvested were calculated using residue factors developed by James O. Howard of U.S. Forest Service (USFS) and harvest data taken from annual Timber Harvest Reports from 1980 to 1988 published by the Washington State Department of Natural Resources (DNR). (Howard, 1981; DNR, 1980-1988) Using Howard's residue factors and DNR's Timber Harvest Reports, quantity of residue generated for each ownership, in each county, were calculated for the years 1981 through 1988 . The average and range of values are reported in this section of the document.

Ratios used were the result of extensive field measurements of the volume of wood residues generated as a result of logging activities. The major elements found to influence residue volume were forest ownership, location (eastern and western Washington), and harvest type (clear cut or partial cut). Appendix B contains the values for each year by owuership for Spokane county; information on other cousties can be obtained from the Washington State Energy Office.

The quantity available for energy depends on environmental and economic factors that are site specific and not quantified in this document. There is also competition for logging residues to be used as raw materials for pulp production or other fiber uses.

Washington state generates nearly 4.2 million dry tons of logging residues annually. Pages I-45 through I-61 show the amount of logging residues generated and potentially available for energy in increasing levels of detail.

All residue generated is assumed available for energy. Over 56 percent comes from private lands, 24 percent from national forests, 13 percent from state lands, 6 percent from Indian lands, and 1 percent from other federal and non-federal lands (see Figure I-5).

Figures I- 6 through I-11 show the amount of logging residues generated in the state from 1981 to 1988 . The total logging residues generated on all land ownerships is summarized in Figure I-6. Figures I-7 through I-11 show logging residues by type of land ownership.

Tables I-25 through I-30 show residues generated by region and land ownership. Maps I-11 through I-16 show the annual average of logging residues by region and by land ownership. The heavily forested areas of the West and Southwest regions generate more than half of the forest residues in this state. The majority of these forests are privately owned.

The counties generating the most residues are Lewis, Grays Harbor, Cowlitz, Jefferson, Skamania. Lewis and Grays Harbor generate 20 percent, which is the greatest percentage for any two counties in the state. Tables I-31 through I-36 show logging residues generated by county and by land ownership. 
Future Availability and Costs

\section{Mill Residues}

The quantity of logging iscidue generated in the next 20 years will depend upon the harvest levels and quantity of residue generated per volume of harvested materials. A decrease in the quantity of logging residues is expected as less and less old growth material is available to be harvested.

Calculating the amount of logging residue that can be delivered to a specific user at a specific cost is a difficult task. A report prepared for the Pacific Northwest and Alaska Bioenergy Program gives logging residue supply curves for Washington state. This study serves as the basis for the cost data derived in this section (U.S. Department of Energy, 1989).

When retrieving residues from the forests, the lowest cost materials are assumed to be removed first, and each additional volume costs more to recover. Firewood is considered to be a less expensive material to retrieve and is usually removed first.

The quantity of logging residue was calculated that could be recovered up to marginal cost of $\$ 3.30 / \mathrm{MBtu}$, chipped and delivered 50 miles to a potential energy site. Table I-37 shows the predicted quantity of material that may be available in Washington state for three different time periods in the future at a marginal cost of less than $\$ 3.30 / \mathrm{MBtu}$. The minimum cost of logging residues is $\$ 2.60 / \mathrm{MBtu}$ and the average would depend upon the specific supply zone. Additional materials would be available at increased costs. The costs given are based on the residues removed paying their own way.

If slash burning is regulated more severely in this state, logging residues may have to be removed to meet environmental regulations. In that case, residue recovery costs may be partially paid for by the commercial timber product. If firewood use is restricted as a secondary source of heat in the residential sector, the quantity removed may decline. This would make more of the less expensive material available and thus reduce the average cost for forest residues.

The method of recovering timber from the forest may change in the future with more whole tree harvesting. This will also result in reduced costs because the cost of bringing the material to the loading point will be paid for by the commercial timber. The effect of future changes in logging practices and the environmental changes on costs of recovery is assumed to balance out resulting in no real change in recovery costs in future years.

Sawmills, shake and shingle mills, and veneer and plywood mills are considered to be the primary wood processing industries. Residues are a combination of scraps, sawdust, and bark from processing whole logs into lumber, plywood, shakes, and shingles. 
The quantity of residues generated depends upon products produced by these mills and the residue factor for each product. The demand for lumber products from Washington state is driven by the world economy and our ability to competitively market our products.

The characteristics of the residues produced depend on the industry. For the most part, sawmills generate sawdust from harvested wood; furniture manufacturers and cabinetmakers primarily generate scraps of veneers, plywood, particle board, and laminates; and shake and shingle mills tend to generate a combination of bark, sawdust, and scraps.

Traditionally, mills have been effective at finding end uses for their waste-over 96 percent are used. Higher grade residues are sold to pulp and paper mills. Lower grade residues are burned on-site to meet the facility's heat and process steam needs or sold to other companies for fuel.

The annual average was based on data spanning the last 10 years and was taken directly from annual mill surveys published by DNR. Washington Mill Survey reports total residues by industry, current use, and how much goes unused. They also report information on industry characteristics and wood consumption by ownership, timber age, and species.

DNR's economic areas differed from the waste generation areas defined by the Department of Ecology. DNR based the economic areas on wood consumption and production of wood products. Since this report is interested in other sources of biomass as well as wood residues, other criteria were equally important. Per capita income, population, employment in lumber and non-lumber industries, agriculture, precipitation, and transportation costs are important aspects to consider in determining how much biomass is available for energy in any particular region. These criteria were already studied by the Department of Ecolosgy when it selected its waste generation areas and are used throughout this report for the biomass regions. For comparison purposes, DNR's regions are listed below (DNR, 1988) and the Department of Ecology's areas are listed on page I-1.

\section{DNR Economic Area}

Olympic Peninsula

Puget Sound

Lower Columbia

\section{Counties}

Clallam, Grays Harbor, Jefferson, Lewis, Mason, Pacific, and Thurston

Island, King, Kitsap, Pierce, San Juan, Skagit, Snohomish, and Whatcom

Cowlitz, Clark, Klickitat, Skammañia, Wahkiakimin 
Central Washington

Inland Empire
Adams, Benton, Chelan, Douglas, Franklin, Grant, Kittitas, Klickitat, Okanogan, and Yakima

Asotin, Columbia, Ferry, Garfield, Pend Oreille, Spokane, Stevens, Whitman, Walla Walla

In order to avoid disclosure, DNR grouped counties having fewer than three operations with other counties. Counties were combined with those used in previous surveys to allow for some comparison. Residues were only reported by county for those counties having greater than three operations.

In order to determine veneer and plywood mill residues by region, a percentage of residues was calculated based on operating capacity of the facilities in the region. Facility operating capacity figures were taken from the 1988 Directory of the Forest Products Industry (Miller Freeman Publications, 1988). As disclosure may be an issue, the data in this report were calculated and presented by region. The data provided are WSEO's best estimate of mill residues for the region.

\section{Current Supply}

Future Availability and Cost
Pages I-63 through I-73 show the supply of mill residues. Figures I-12 through I-15 show mill residues by type of mill during the last 10-year period for which data are available, 1978 through 1988. Historically, mills sold and used a relatively high percentage of residues generated, only a small percentage have gone unused. In 1988, for example, 47 percent was used for pulp and board, 41 percent was used as fuel, 9 percent was used for other purposes, and only 2 percent went unused.

From 1978 through 1988 the sawmill, veneer and plywood, and shake and shingle industries averaged over 5.1 million dry tons of wood and bark residues annually. Sawmills accounted for close to 82 percent, veneer and plywood mills generated 15 percent, and shake and shingle mills generated only 3 percent. (See Tables I-38 through I-45.)

Maps I-17 through I-24 clearly illustrate that Western Washington dominates the state in the amount of residues generated. Table I. 38 shows the Southwest region generated 1.34 million dry tons and the Puget Sound region generated approximately 1.26 million dry tons. The West ranked third generating over 926 thousand dry to:us annually. Table I-39 shows the unused portion of the amrunt generated.

The calculated quantity of available mill residues is highly dependent upon the accuracy of the data used. Since availability is calculated as the difference between two numbers (supply and demand) that are approximateiy equai, an error in estimating either suppiy or demand will result in a relatively large change in the calculated 
amount available. The quantity of residue generated can be determined more precisely than demand because of accepted residue factors and records kept on primary production. The large use of residues by pulp and board industries for furnish and fuel is the major demand sector. Predicting their future demands for residues is difficult and has not been attempted for this report.

The cost of mill resides depends upon the balance between supply and demand. When supply is greater than demand, the material is generally available for the cost of transportation. The average cost of material at a particular energy site depends upon how far the material must be transported. Table 1-3, page I-15 shows the iransportation costs per ton at different haul distances.

Table I-4ú shows the predicted quantity generated and available for the cost of transportation for each time period for the two demand assumptions. The quantities generated in the future were computed by using the Resource Information Systems, Inc. (RISI) projections for the quantity of lumber and plywood that would be produced between 1991 and 2010 (RSI, 1988). The demand for fuels was assumed to be the same as determined by the surveys conducted by the Washington State Energy Office. The quantity of material going to pulp and board was assumed to be either 1) the historical percentage of residues generated that go to pulp and board or 2) the use of all the course residues generated (Larson, 1986; Howard, 1988).

The quantities listed above as available for fuel may still have to compete with other residue uses, such as animal bedding or landscape. For example, landscaping bark in the Puget Sound was selling for $\$ 44 /$ ton in June 1989 . The effect of future changes in the residue factor due to higher recovery rates and changing size and quality of timber was not considered. The quantity of material available with a reasonable supply radius will determine the quantity of material that can be used for electric generation.

\section{Secondary Wood Processing}

Secondary wood processing industries use lumber produced from mills to manufacture their products. Residues are primarily made up of sawdust, shavings, fines, and chips. Manufacturers of structural wood members (trusses, arches, timbers), pallets, skids, boxes, shook, barrels, baskets, premanufactured housing, and furniture are considered secondary wood processing industries.

Information in this section came directly from WSEO's 1990 Wood Residue Survey and 1990 Directory of Secondary Wood Processing Facilities in Washington State, 1990. For this assessment, WSEO surveyed eight secondary lumber and wood products manufacturing industries: hardwood dimension and flooring, millwork, wood kitchen cabinets, structural wood members (not classified elsewhere), nailed wood and shook boxes, wood pallets and skids, wood mobile homes, and prefabricated wood building facilities. 


\section{Current Supply}

\section{Future Availability and Costs}

\section{Sources}

Many businesses did not keep accurate records of how much residue they generated in 1989 or how it was used. As a result, rough estimates were given and may not be accurate.

Altempts were made to estimate the total resıdue generated based on the figures given by the firms. Ratios computed for residue per board foot consumed and residues per employee varied considerably by both industry and employment size classifications. Rather than take the chance of significantly overestimating or underestimating the total, only the actual amount reported is given (WSEO, 1990b).

In 1989, 169,899 dry tons of residues were reported statewide by secondary wood processing facilities. Of that, 34 percent can potentially be used for energy. The Puget Sound region dominated for both residues generated and residues unused. Pierce, Snohomish, Cowlitz, King, and Spokane counties were among the top generators. (See Maps I-25 through I-26 and Tables I-47 through I-50, on pages 1-75 through I-78).

Future residues available for energy will depend on demand for products and competition for residues.

Howard, J.O. Ratios for Estimating Logging Residue in the Pacific Northwest. 1981.

Howard, J.O. and F.R. Ward. Oregon's Forest Products Industry 1985.1988.

Larson, D.N. and D.A. Bearden. 1984 Mill Survey. 1986.

The Matrix Management Group, R.W. Beck and Associates, Gilmore Research Group. Best Management Practices Analysis for Solid Waste, 1987 Recycling and Waste Stream Survey, Volume 1. Prepared for the Office of Waste Reduction and Recycling, Washington State Department of Ecology. December 1988.

Miller Freeman Publications. 1988 Directory of the Forest Products Industry. 1988.

Resource Information Systems, Inc. Supply, Demand, and Prices of Pacific Northwest Timber, Volume I: An Assessment of Market Opportunities in an Environment of Increasing Competition. 1988.

Washington Agricultural Statistics Service. Washington Agricultural Statistics. 1982 through 1989.

Washington State Department of Natural Resources. Timber Harvest Reports. 1980 through 1988.

Washington State Department of Natural Resources. 1988 Washington Mill Survey. Pages D-14 \& 15. 1991. 
Washington State Energy Office. 1990 Washington State Directory of Biomass Energy Facilities. Page 13. 1990a.

Washington State Energy Office. 1990 Wood Residue Survey and Directory of Secondary Wood Processing Facilities in Washington State. $1990 \mathrm{~b}$.

U.S. Department of Energy. Biomass Project Development Guidebook. Prepared for the Pacific Northwest and Alaska Bioenergy Program. 1989. 
I

,

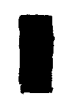

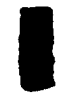

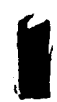

\section{Biomass}

\section{Tables}


Table 1-1

Future Availability and Price of Biomass Fuels

Residue Resource

Trillion Btulyear

Price, \$MBtu

Logging Residue

$36-48$

$2-7$

$2.60-3.30$

Mill Residues

$15-38$

$0.40-1.00$

Agricultural Field Residue

$35-40$

2.20

Municipal Solid Waste

-5.00 to -8.00

Table 1-2

\section{Factors Determining Cost of Residues}

Resource

Price Determinants

Logging Residue

Collection cost; transportation

Collection cost; transportation

Wood Products

Altemative disposal cost; transportation

Municipal Solid Waste

Altemative disposal cost; transportation

Table 1-3

Transportation Costs - UTC Rates

\begin{tabular}{llr}
\hline Miles & \$/Ton & \$MBtu \\
\hline & & \\
10 & & 0.31 \\
20 & 2.62 & 0.43 \\
30 & 3.66 & 0.55 \\
40 & 4.72 & 0.66 \\
50 & 5.64 & 0.77 \\
75 & 6.60 & 1.07
\end{tabular}

Sourc: WA State Utilities and Transportation Tariff No. 4-A, 1989 


\section{Agricultural Residues}

Figures, Maps, and Tables 
Figure 1-1

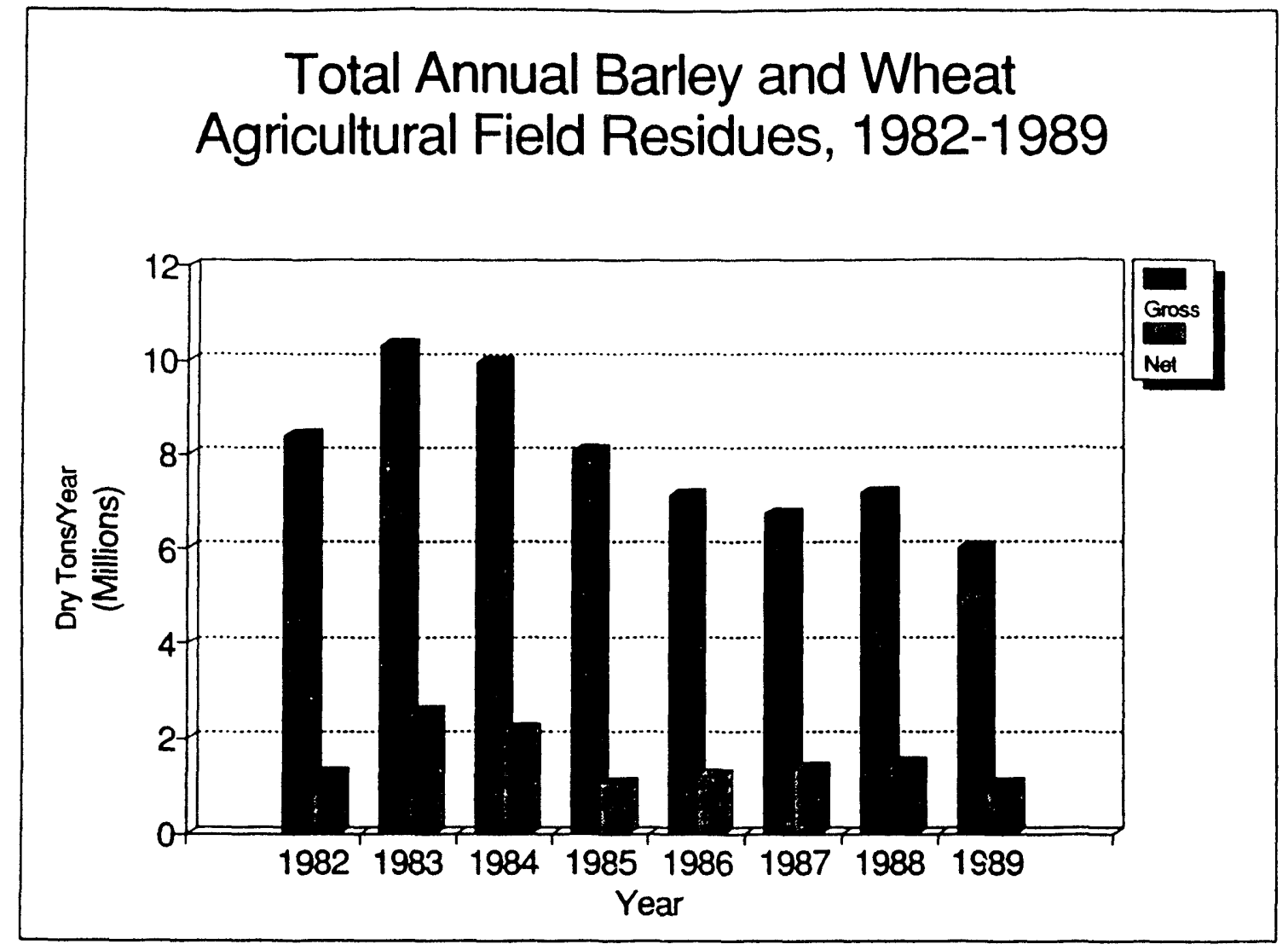

Figure 1-2

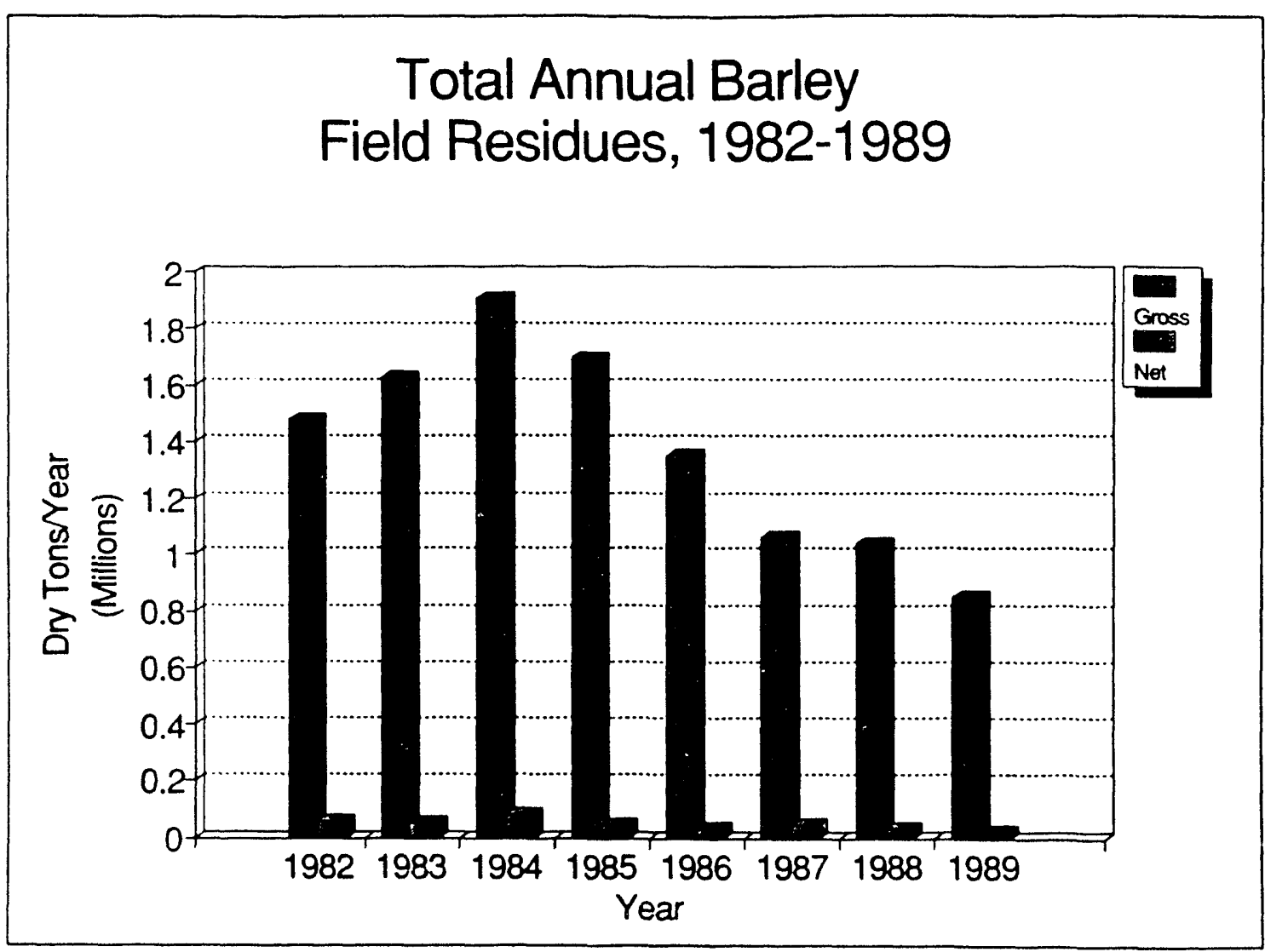


Figure I-3

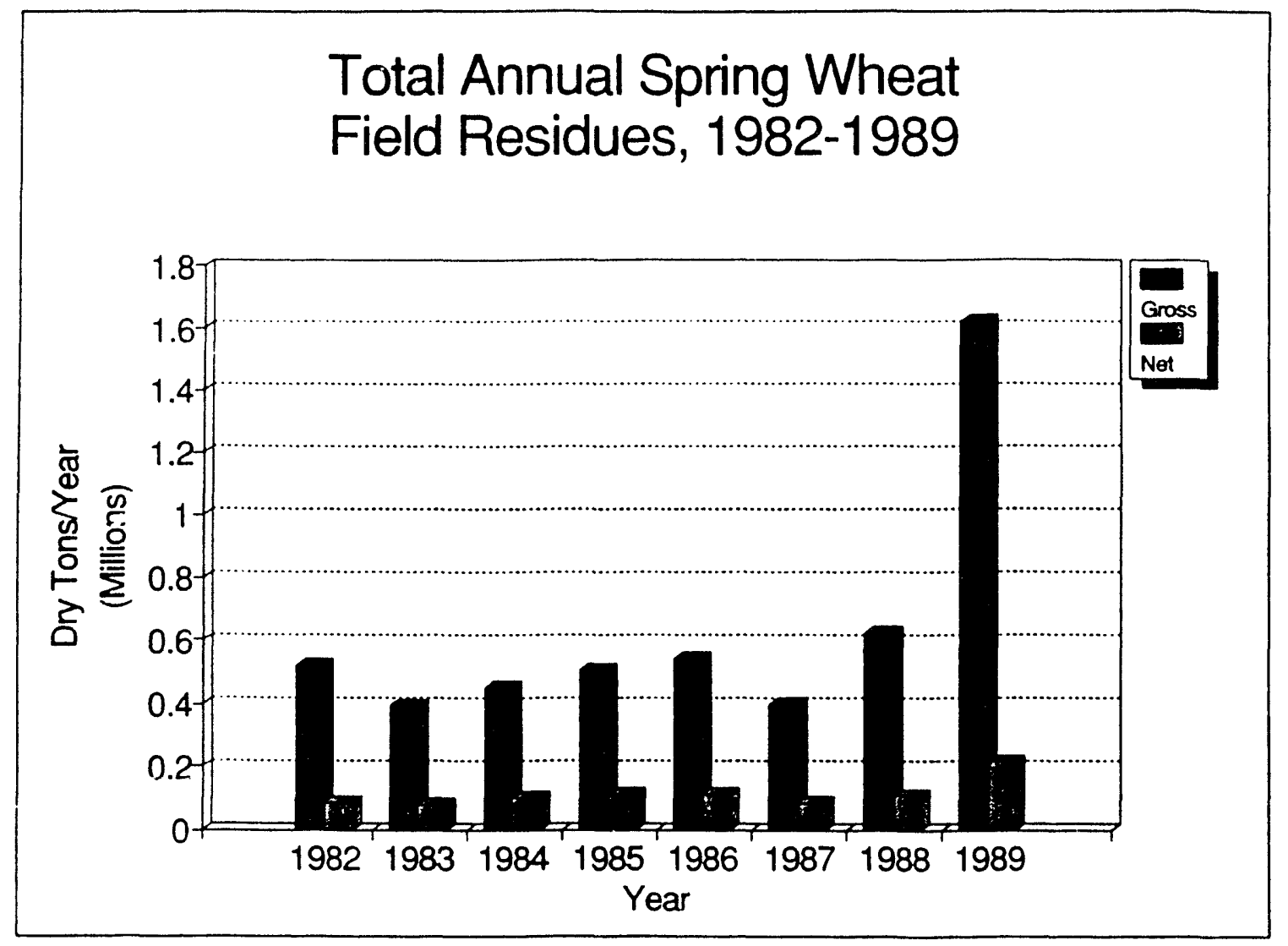

Figure l-4

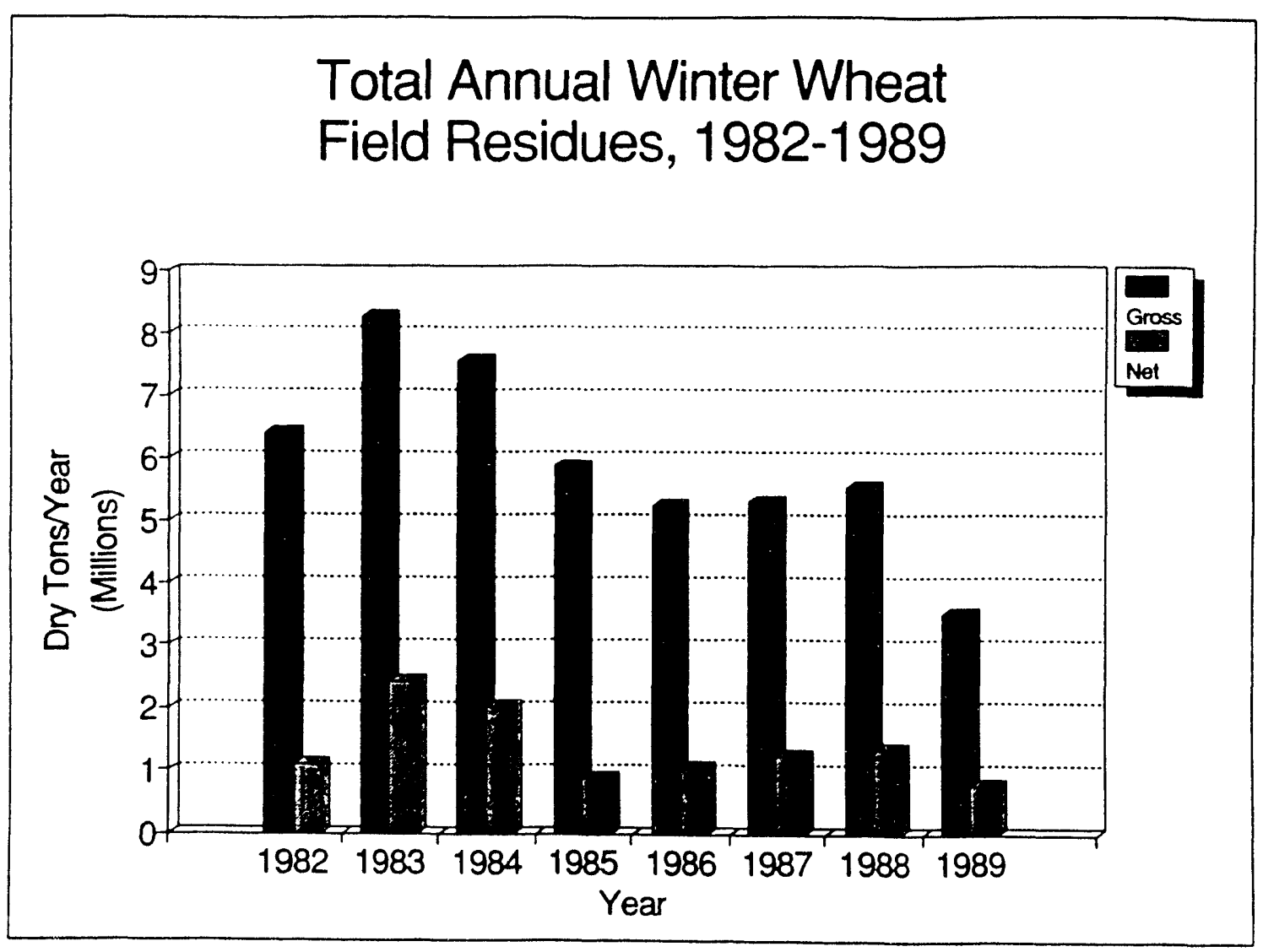


Map I-1

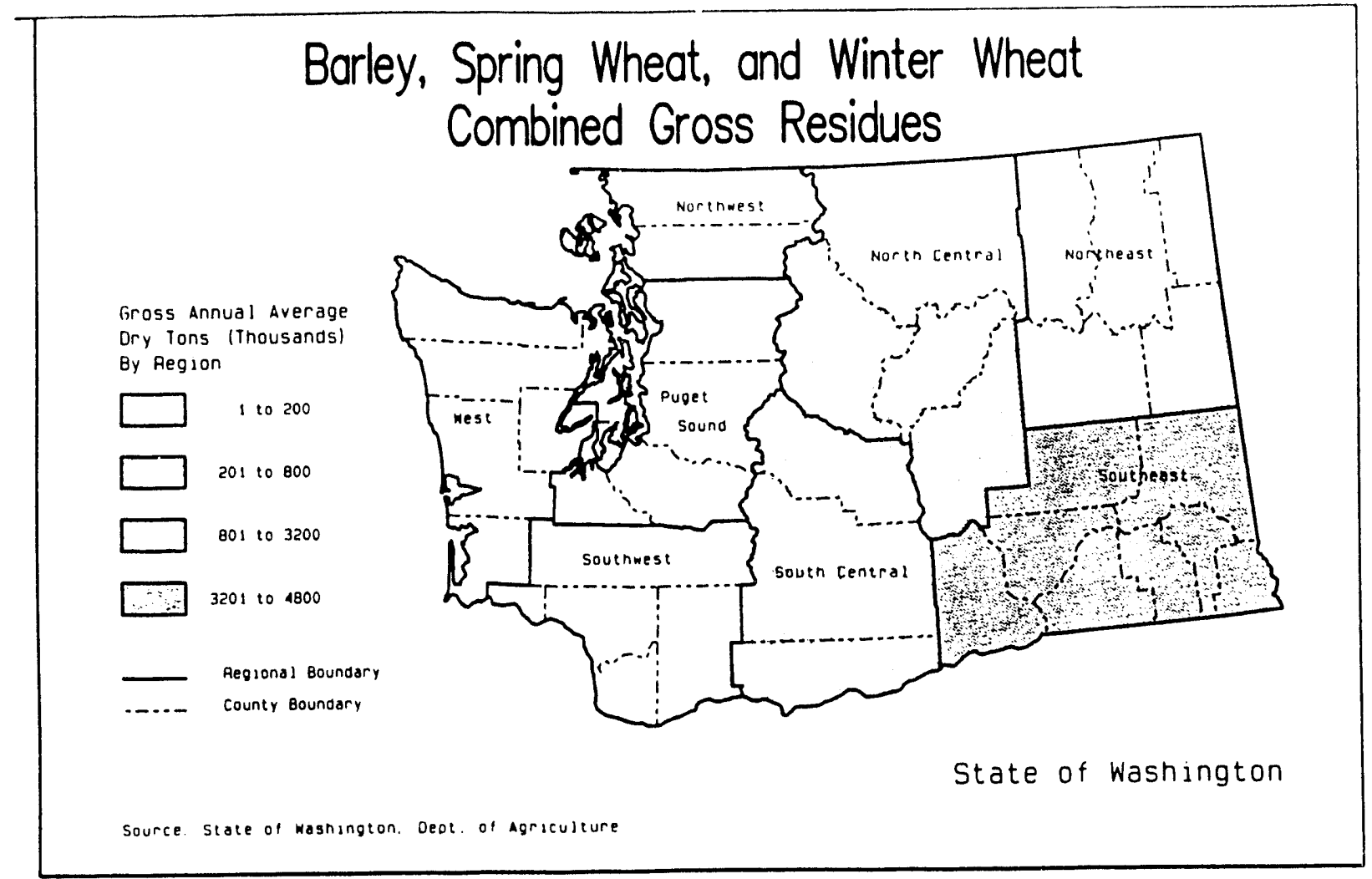

Map 1-2

\section{Barley, Spring Wheat, and Winter Wheat} Combined Net Residues

Net Annual Average

Ory Tons (Thousands) By Region

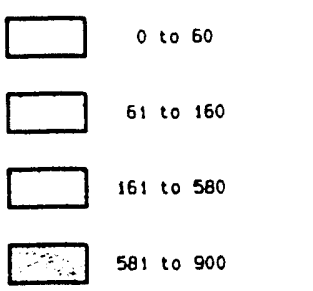

- Regional Bounoary -...- County Boundary

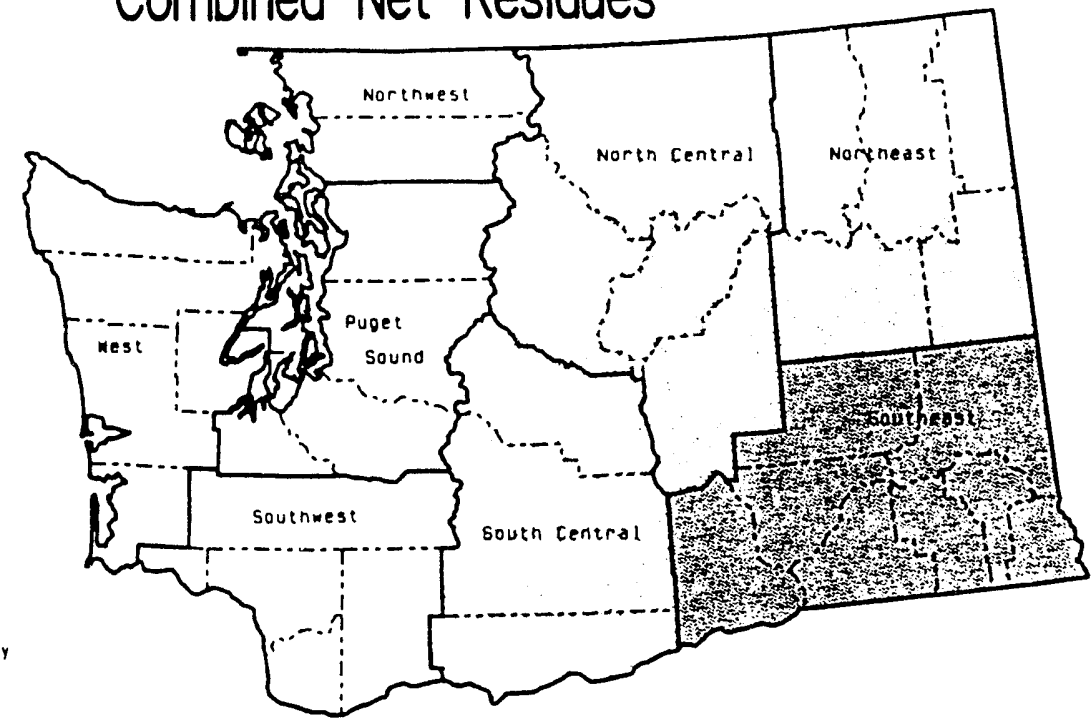

State of Washington 
Map 1-3

\section{Gross Barley Residues}

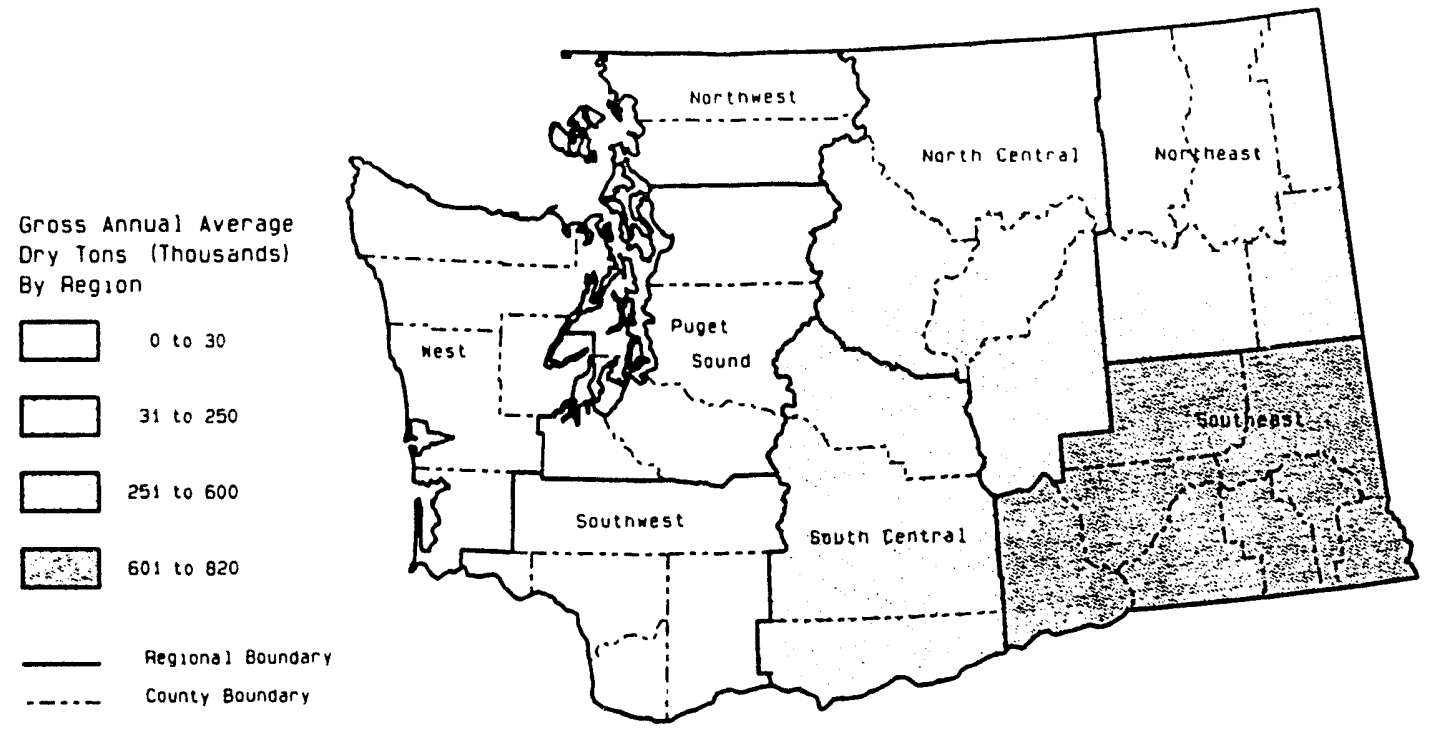

State of Washington

Source. State of Washington. Dedt. of Agriculture

Map 1-4

\section{Net Barley Residues}

Net Annual Average

Dry Tons (Hundreds)

By Region

$\square 0103$

$\square .1040$

$\square 1110110$

\begin{tabular}{llll}
\hline & 111 & 10 & 160
\end{tabular}

- Regional Boundary

-...- County Bounoary

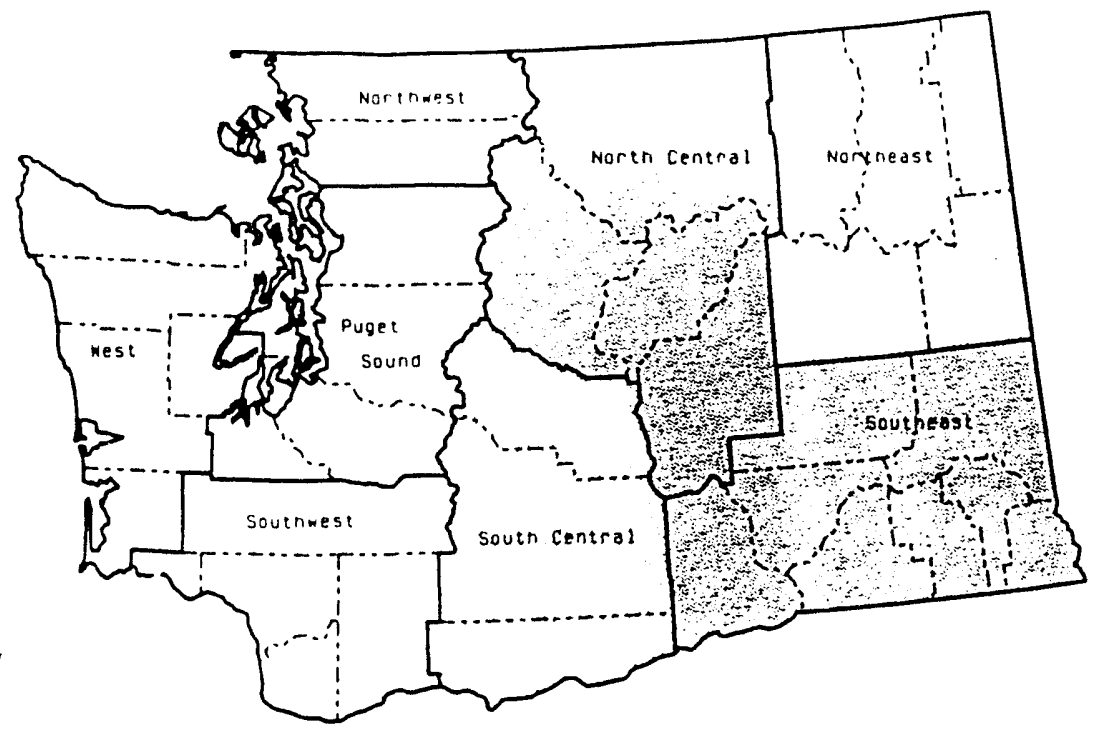

State of Washington 


\section{Map 1-5}

\section{Gross Spring Wheat Residues}

Gross Annual Average Ory Tons (Thousands) By Region

$\square 01035$

$\square 36$ 10 95

$\square 96220$

22110300

-...- Regional Boundar

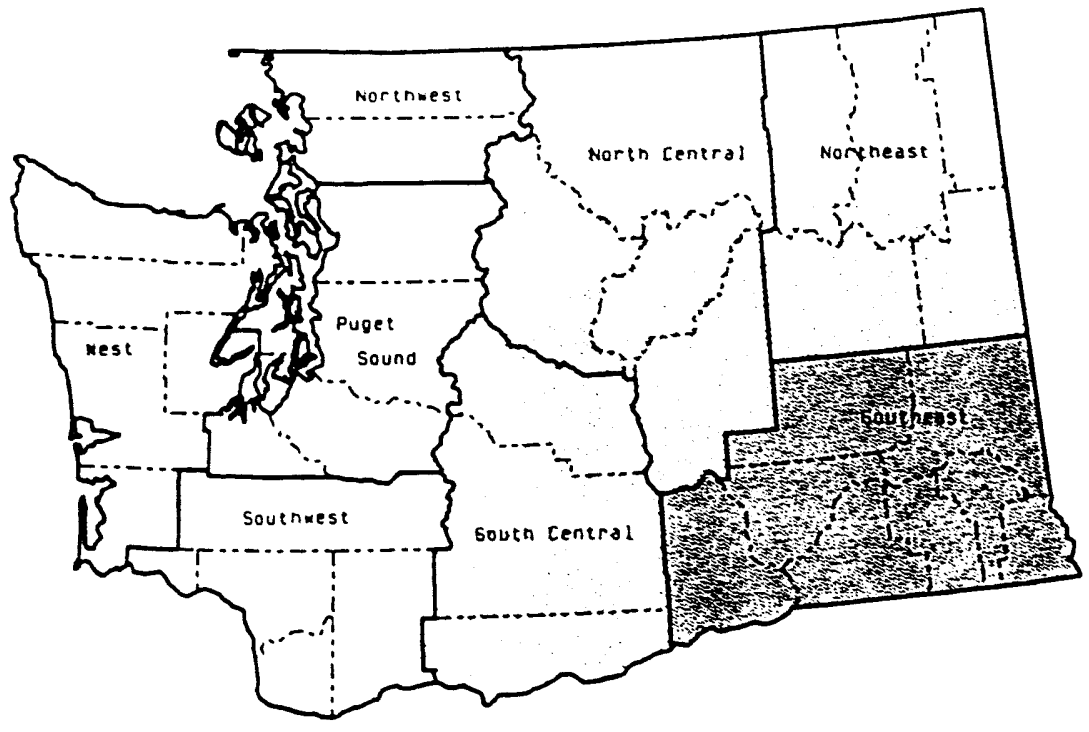

State of Washington

Source state of masnington. Dept. Of agriculture

\section{Map 1-6}

\section{Net Spring Wheat Residues}

Ne: Annual Averaģe

Ory Tons iHunarecs!

E) Fegion

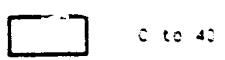

$\square 4: 10150$

$\square 15:$ to 310

$\square 3: 10 \cos$

Lezional soungar

....- Couni, Eungar.

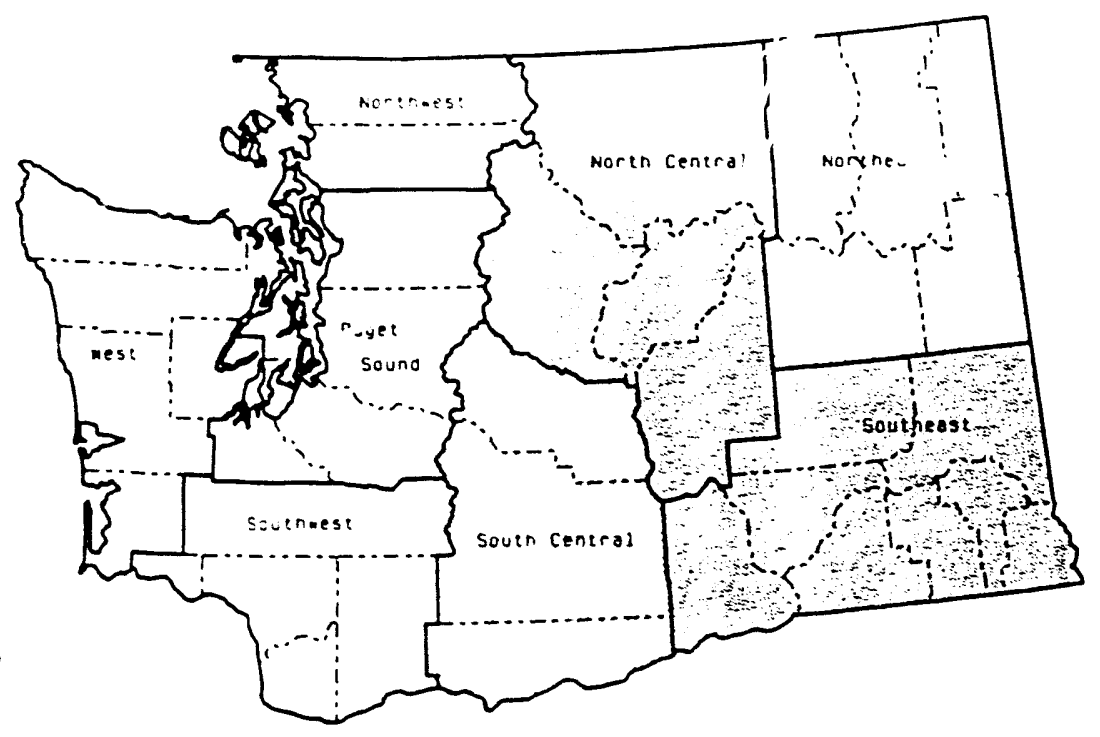

State of Washington 
Map 1-7

\section{Gross Winter Wheat Residues}

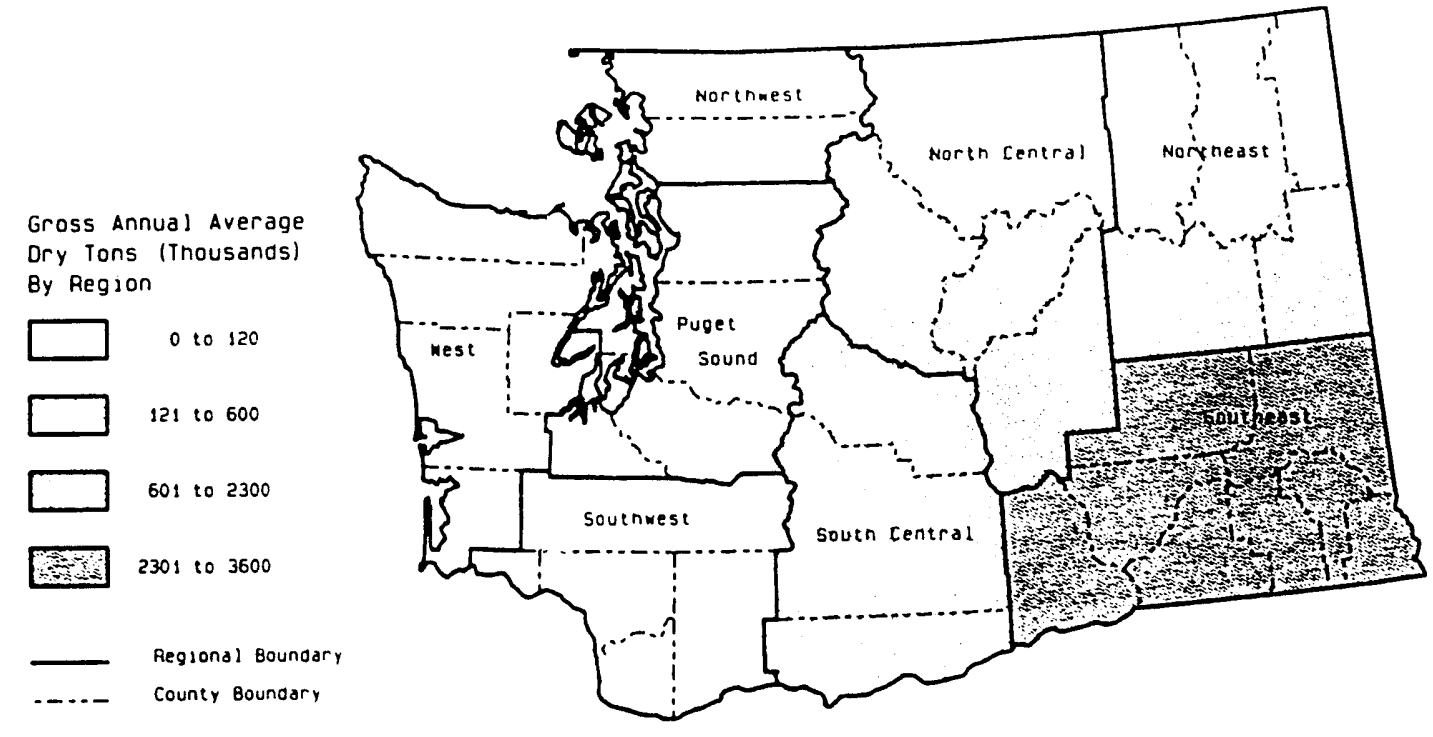

State of Washington

Source. State of masnington. Dedt. of Agriculture

Map I-8

\section{Net Winter Wheat Residues}

Net Annual Average Ory Tons (Tnousandas) By Region

$\square 0$ to 10

$\square$ प1 10140

$\square 14110530$

53110830

- Regional Boundary

.... County Bounoary

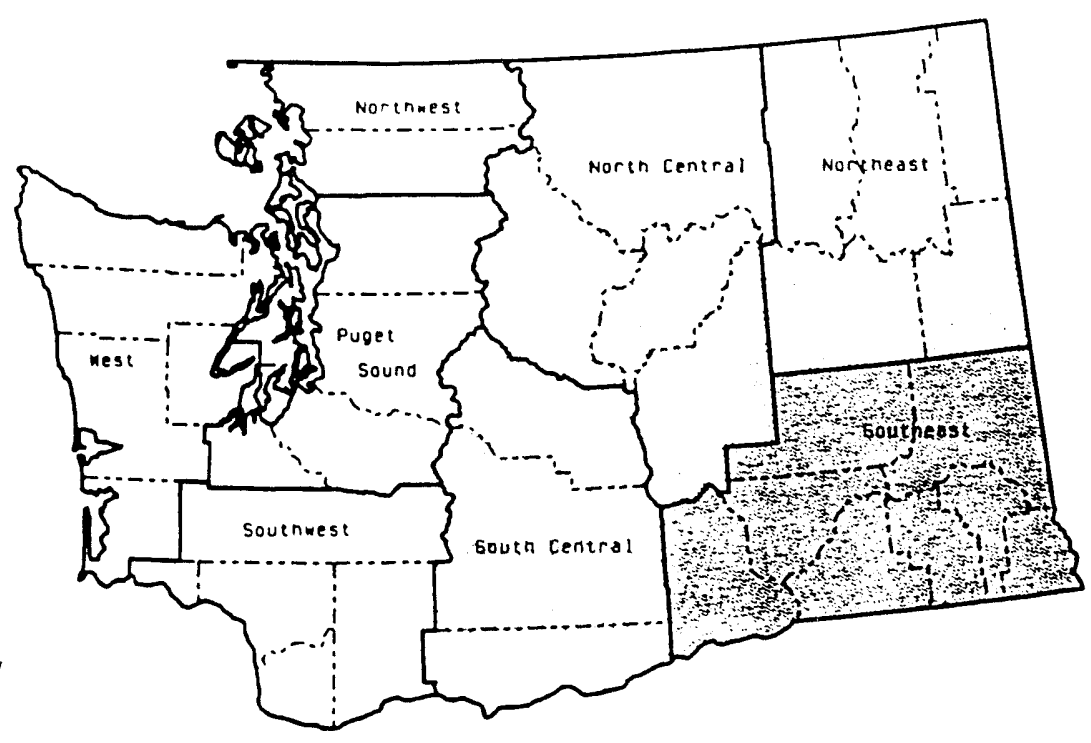

State of Washington

Source state of washington. Depl of Agraculture 
Table 1-4

Gross Barley, Spring Wheat, Winter Wheat Residues by Region

Ranked by Gross Annual Average (dry tons)

\begin{tabular}{|c|c|c|c|c|c|}
\hline \multirow[b]{2}{*}{ Rank } & \multirow[b]{2}{*}{ Region } & \multicolumn{2}{|c|}{---Gross Annual Average ${ }^{1}$} & \multicolumn{2}{|c|}{ 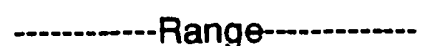 } \\
\hline & & (Dry Tons) $^{2}$ & (M Btu/Year) ${ }^{3}$ & Minimum & Maximum \\
\hline 1 & Southeast & $4,661,360$ & $69,920,395$ & $3,564,105$ & $6,103,917$ \\
\hline 2 & Northeast & $1,660,977$ & $24,914,649$ & $1,130,190$ & $2,206,545$ \\
\hline 3 & North Central & $1,235,681$ & $18,535,218$ & 794,850 & $1,693,389$ \\
\hline 4 & South Central & 356,604 & $5,349,054$ & 211,872 & 523,680 \\
\hline 5 & Northwest & 35,798 & 536,963 & 12,789 & 65,955 \\
\hline 6 & Southwest & 18,305 & 274,579 & 6,171 & 36,393 \\
\hline 7 & Puget Sound & 7,758 & 116,376 & 1,383 & 17,790 \\
\hline \multirow[t]{2}{*}{8} & West & 1,419 & 21,285 & 0 & 5,961 \\
\hline & State & $7,977,901$ & $119,668,519$ & $5,991,306$ & $10,306,260$ \\
\hline
\end{tabular}

1 Average based on reports from 19:82 through 1989.

2 Includes residues from both dry and irrigated lands.

3Energy equivalent, 15 million Btu/ton.

Source: Washington State Dept. of Agriculture, Washington Agricultural Statistics, 1982-1989.

Table 1-5

\section{Net Barley, Spring Wheat, Winter Wheat Residues by Region}

Ranked by Net Annual Average (dry tons)

\begin{tabular}{|c|c|c|c|c|c|}
\hline \multirow[b]{2}{*}{ Rank } & \multirow[b]{2}{*}{ Region } & \multicolumn{2}{|c|}{-...-Gross Annual Average ${ }^{1} \ldots$} & \multicolumn{2}{|c|}{ - } \\
\hline & & (Dry Tons) $^{2}$ & (M Btu/Year) $^{3}$ & Minimum & Maximum \\
\hline 1 & Southeast & 874,877 & $13,123,149$ & 398,229 & $1,611,552$ \\
\hline 2 & Northeast & 227,582 & $3,413,724$ & 36,080 & 518,080 \\
\hline 3 & North Central & $28^{-n 72}$ & $4,317,035$ & 210,830 & 445,538 \\
\hline 4 & South Central & 11. 29 & $1,507,436$ & 39,951 & 181,290 \\
\hline 5 & Northwest & $\therefore . .33$ & 174,499 & 1,819 & 25,475 \\
\hline 6 & Southwest & 2,828 & 42,413 & 569 & 9,312 \\
\hline 7 & Puget Sound & 1,032 & 15,476 & 0 & 5,138 \\
\hline \multirow[t]{2}{*}{8} & West & 62 & 934 & 0 & 461 \\
\hline & State & $1,506,311$ & $22,594.666$ & 1.003 .240 & 2.554 .574 \\
\hline
\end{tabular}

1 Average based on reports from 1982 through 1989.

2 Includes residues from both dry and irrigated lands.

3 Energy equivalent, 15 million Btu/ton.

Source: Washington State Dept. of Agriculture, Washington Agricultural Statistics, 1982-1989. 
Table 1-6

\section{Gross Barley Residues by Region}

Ranked by Gross Annual Average (dry tons)

\begin{tabular}{|c|c|c|c|c|c|}
\hline \multirow[b]{2}{*}{ Rank } & \multirow[b]{2}{*}{ Region } & \multicolumn{2}{|c|}{...-Gross Annual Average 1 -... } & \multicolumn{2}{|c|}{ 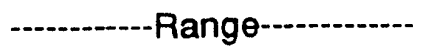 } \\
\hline & & (Dry Tons) $^{2}$ & (M Btu/Year) $^{3}$ & Minimum & Maximum \\
\hline 1 & Southeast & 811,115 & $12,166,725$ & 504,147 & $1,116,360$ \\
\hline 2 & Northeast & 402,643 & $6,039,645$ & 268,731 & 558,774 \\
\hline 3 & North Central & 93,074 & $1,396,110$ & 38,928 & 160,536 \\
\hline 4 & South Central & 51,947 & 779,205 & 28,794 & 73,830 \\
\hline 5 & Northwest & 9,102 & 136,530 & 5,010 & 14,988 \\
\hline 6 & Southwest & 8,034 & 120,510 & 2,475 & 12,780 \\
\hline 7 & Puget Sound & 1,049 & 15,735 & 630 & 1,488 \\
\hline 8 & West & 727 & 10,905 & 0 & 1,710 \\
\hline & State & $1,377,689$ & $20,665,365$ & 851,580 & $1,909,998$ \\
\hline
\end{tabular}

1 Average based on reports from 1982 through 1989.

2 Includes residues from both dry and irrigated lands.

3 Energy equivalent, 15 million Btu/ton.

Source: Washington State Dept. of Agriculture, Washington Agricultural Statistics, 1982-1989.

Table 1-7

\section{Net Barley Residues by Region}

Ranked by Net Annual Average (dry tons)

\begin{tabular}{|c|c|c|c|c|c|}
\hline \multirow[b]{2}{*}{ Rank } & \multirow[b]{2}{*}{ Region } & \multicolumn{2}{|c|}{-.--Net Annual Average1..... } & \multicolumn{2}{|c|}{ 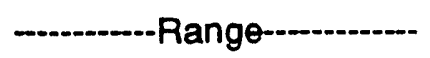 } \\
\hline & & (Dry Tons) $^{2}$ & $(\mathrm{M} \mathrm{Btu} / \text { Year })^{3}$ & Minimum & Maximum \\
\hline 1 & North Central & 15,213 & 228,195 & 5,286 & 25,335 \\
\hline 2 & Southeast & 12,929 & 193,935 & 6,088 & 28,630 \\
\hline 3 & Northeast & 8,809 & 132,135 & 3,059 & 19,584 \\
\hline 4 & South Central & 7,663 & 114,945 & 4,350 & 13,790 \\
\hline 5 & Northwest & 573 & 8,595 & 260 & 805 \\
\hline 6 & West & 26 & 390 & 0 & 210 \\
\hline 7 & Puget Sound & 16 & 240 & 0 & 130 \\
\hline 8 & Southwest & 8 & 120 & 0 & 50 \\
\hline & State & 45,237 & 678,555 & 19,700 & 78,255 \\
\hline
\end{tabular}

1 Average based on reports from 1982 through 1989.

2 includes residues from both dry and irrigated lands.

3 Energy equivalent, 15 million Btu/ton.

Source: Washington State Dept. of Agriculture, Washington Agricultural Statistics, 1982-1989. 
Table 1-8

\section{Gross Spring Wheat Residues by Region}

Ranked by Gross Annual Average (dry tons)

\begin{tabular}{|c|c|c|c|c|c|}
\hline \multirow{2}{*}{\multicolumn{2}{|c|}{ Rank Region }} & \multicolumn{2}{|c|}{---Gross Annual Average ${ }^{1}$} & \multicolumn{2}{|c|}{ 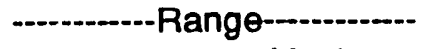 } \\
\hline & & (Dry Tons) $^{2}$ & $\left(M \mathrm{Btu} / \mathrm{Year}^{3}\right)^{3}$ & Minimum & Maximum \\
\hline 1 & Southeast & 293,286 & $4,399,283$ & 114,816 & 898,443 \\
\hline 2 & North Central & 146,523 & $2,197,845$ & 120,244 & 206,271 \\
\hline 3 & Northeast & 120,632 & $1,809,480$ & 34,593 & 438,750 \\
\hline 4 & South Central & 66,831 & $1,002,465$ & 30,348 & 137,046 \\
\hline 5 & Puget Sound & 4,236 & 63,540 & 0 & 15,600 \\
\hline 6 & Northwest & 1,087 & 16,305 & 0 & 3,081 \\
\hline 7 & West & 692 & 10,380 & 0 & 4,251 \\
\hline 8 & Southwest & 327 & 4,905 & 0 & 1,560 \\
\hline & State & 633,614 & $9,504,203$ & 392,496 & $1,625,520$ \\
\hline
\end{tabular}

${ }^{1}$ Average based on reports from 1982 through 1989.

2 Includes residues from both dry and irrigated lands.

3 Energy equivalent, 15 million Btu/ton.

Source: Washington State Dept. of Agriculture, Washington Agricultural Statistics, 1982-1989.

Table 1-9

\section{Net Spring Wheat Residues by Region}

Ranked by Net Annual Average (dry tons)

\begin{tabular}{|c|c|c|c|c|c|}
\hline \multirow[b]{2}{*}{ Rank } & \multirow[b]{2}{*}{ Region } & \multicolumn{2}{|c|}{-Not Annual Average ${ }^{1} \ldots$} & \multicolumn{2}{|c|}{ 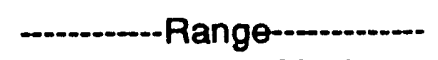 } \\
\hline & & (Dry Tons) $^{2}$ & $(M \text { Btu/Year })^{3}$ & Minimum & Maximum \\
\hline 1 & Southeast & 41,907 & 628,605 & 22,760 & 117,026 \\
\hline 2 & North Central & 39,526 & 592,890 & 33,007 & 52,600 \\
\hline 3 & South Central & 22,264 & 333,960 & 9,965 & 43,635 \\
\hline 4 & Northeasi & 8,064 & 120,960 & 321 & 19,872 \\
\hline 5 & Northwest & 77 & 1,155 & 0 & 331 \\
\hline 6 & Puget Sound & 73 & 1,095 & 0 & 393 \\
\hline 7 & West & 36 & 540 & 0 & 251 \\
\hline \multirow[t]{2}{*}{8} & Southwest & 0 & 0 & 0 & 0 \\
\hline & State & 111,947 & 1.679 .205 & 80,786 & 217,395 \\
\hline
\end{tabular}

1 Average based on reports from 1982 through 1989.

2 Includes residues from both dry and irrigated lands.

${ }^{3}$ Energy equivalent, 15 million Btu/ton.

Source: Washington State Dept. of Agriculture, Washington Agricultural Statistics, 1982-1989. 
Table $1-10$

\section{Gross Winter Wheat Residues by Region}

Ranked by Gross Annual Average (dry tons)

\begin{tabular}{|c|c|c|c|c|c|}
\hline \multirow{2}{*}{\multicolumn{2}{|c|}{ Rank Region }} & \multirow{2}{*}{\multicolumn{2}{|c|}{$\begin{array}{l}\text {-..-Gross Annual Average }{ }^{1} \ldots- \\
\text { (Dry Tons) }^{2} \quad \text { (M Btu/Year) }^{3}\end{array}$}} & \multicolumn{2}{|c|}{ 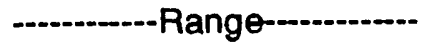 } \\
\hline & & & & Minimum & Maximum \\
\hline 1 & Southeast & $3,556,960$ & $53,354,400$ & $2,271,234$ & $5,018,247$ \\
\hline 2 & Northeast & $1,137,702$ & $17,065,530$ & 514,845 & $1,547,850$ \\
\hline 3 & North Central & 996,084 & $14,941,260$ & 549,729 & $1,409,538$ \\
\hline 4 & South Central & 237,826 & $3,567,390$ & 0 & 420,495 \\
\hline 5 & Northwest & 25,608 & 384,120 & 3,825 & 49,623 \\
\hline 6 & Southwest & 9,945 & 149,175 & 3,060 & 23,613 \\
\hline 7 & Puget Sound & 2,091 & 31,365 & 0 & 12,138 \\
\hline \multirow[t]{2}{*}{8} & West & 0 & 0 & 0 & 23,613 \\
\hline & State & $5,966,216$ & $89,493,240$ & $3,511,146$ & $8,284,032$ \\
\hline
\end{tabular}

1 Average based on reports from 1982 through 1989.

2 Includes residues from both dry and irrigated lands.

3 Energy equivalent, 15 million Btutton.

Source: Washington State Dept. of Agriculture, Washington Agricultural Statistics, 1982-1989.

Table I-11

\section{Net Winter Wheat Residues by Region}

Ranked by Net Annual Average (dry tons)

\begin{tabular}{|c|c|c|c|c|c|}
\hline \multirow{2}{*}{\multicolumn{2}{|c|}{ Rank Region }} & \multicolumn{2}{|c|}{...-Net Annual Average 1 -..... } & \multicolumn{2}{|c|}{ - } \\
\hline & & (Dry Tons) $^{2}$ & (M Btu/Year) $^{3}$ & Minimum & Maximum \\
\hline 1 & Southeast & 820,040 & $12,300,600$ & 431,054 & $1,542,633$ \\
\hline 2 & North Central & 233,064 & $3,495,960$ & 150,280 & 370,816 \\
\hline 3 & Northeast & 210,709 & $3,160,635$ & 49,001 & 480,600 \\
\hline 4 & South Central & 70,568 & $1,058,520$ & 0 & 158,077 \\
\hline 5 & Northwest & 10,983 & 164,745 & 1,075 & 24,373 \\
\hline 6 & Southwest & 2,820 & 42,300 & 810 & 9,113 \\
\hline 7 & Fuget Sound & 872 & 13,080 & 0 & 5,138 \\
\hline \multirow[t]{2}{*}{8} & West & 0 & 0 & 0 & 0 \\
\hline & State & $1.349,056$ & 20.233 .840 & 765.585 & 2.420 .285 \\
\hline
\end{tabular}

${ }^{1}$ Average based on reports from 1982 through 1989.

2 includes residues trom both dry and irrigated lands.

3Energy equivalent, 15 million Btu/ton.

Source: Washington State Dept. of Agriculture, Washington Agricultural Statistics, 1982-1989. 
Table 1-12

\section{Gross Barley, Spring Wheat, Winter Wheat Residues by County}

Ranked by Quantity

\begin{tabular}{|c|c|c|c|c|c|}
\hline \multirow[b]{2}{*}{ Rank } & \multirow[b]{2}{*}{ County (Region) } & \multicolumn{2}{|c|}{---Gross Annual Average 1 -... } & \multicolumn{2}{|c|}{ 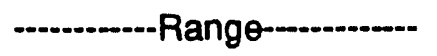 } \\
\hline & & $(\text { Dry Tons })^{2}$ & (M Btu/Year) ${ }^{3}$ & Minimum & Maximum \\
\hline 1 & Whitman (SE) & $1,715,315$ & $25,729,723$ & $1,421,235$ & $2,174,043$ \\
\hline 2 & Lincoln (NE) & $1,127,815$ & $16,917,227$ & 757,416 & $1,453,512$ \\
\hline 3 & Adams (SE) & 923,055 & $13,845,832$ & 651,849 & $1,256,592$ \\
\hline 4 & Grant (NC) & 751,011 & $11,265,164$ & 546,576 & 995,202 \\
\hline 5 & Walla Walla (SE) & 742,896 & $11,143,434$ & 561,495 & 945.975 \\
\hline 6 & Spokane (NE) & 474,198 & $7,112,976$ & 339,522 & 662,301 \\
\hline 7 & Douglas (NC) & 456,998 & $6,854,973$ & 230,895 & 656,133 \\
\hline 8 & Franklin (NE) & 371,342 & $5,570,124$ & 247,317 & 512,418 \\
\hline 9 & Columbia (SE) & 305,647 & $4,584,701$ & 233,418 & 419,109 \\
\hline 10 & Garfield (SE) & 258,862 & $3,882,932$ & 198,054 & 327,618 \\
\hline 11 & Benton (SE) & 256,360 & $3,845,402$ & 189,843 & 344,202 \\
\hline 12 & Yakima (SC) & 208,242 & $3,1,23,636$ & 105,594 & 325,698 \\
\hline 13 & Klickitat (SC) & 113,494 & $1,702,406$ & 92,139 & 143,589 \\
\hline 14 & Asotin (SE) & 87,883 & $1,318,247$ & 60,894 & 123,960 \\
\hline 15 & Stevens (NE) & 49,863 & 747,951 & 31,056 & 70,782 \\
\hline 16 & Kittitas (SC) & 34,868 & 523,013 & 14,139 & 54,393 \\
\hline 17 & Skagit (NW) & 26,748 & 401,226 & 8,871 & 50,712 \\
\hline 18 & Okanogan (NC) & 21,860 & 327,898 & 14,184 & 33,360 \\
\hline 19 & Clark (SW) & 11,712 & 175,686 & 3,582 & 25,233 \\
\hline 20 & Snohomish (PS) & 7,671 & 115,059 & 1,383 & 17,088 \\
\hline 21 & Ferry (NE) & 7,560 & 113,406 & 1,356 & 17,688 \\
\hline 22 & Lewis (SW) & 6,593 & 98,893 & 2,589 & 11,160 \\
\hline 23 & Chelan (NC) & 5,812 & 87,182 & 3,195 & 8,694 \\
\hline 24 & Whatcom (NW) & 5,018 & 75,274 & 1,413 & 8,751 \\
\hline 25 & Island (NW) & 3,487 & 52,307 & 2,505 & 5,148 \\
\hline 26 & Pend Oreille (NE) & 1,539 & 23,091 & 840 & 2,262 \\
\hline 27 & Clallam (W) & 1,419 & 21,285 & 0 & 5,961 \\
\hline 28 & San Juan (NW) & 544 & 8,156 & 0 & 1,344 \\
\hline 29 & Pierce (PS) & 88 & 1,316 & 0 & 702 \\
\hline NA & Cowlitz (SW) & 0 & 0 & 0 & 0 \\
\hline NA & Grays Harbor (W) & 0 & 0 & 0 & 0 \\
\hline NA & Jefferson $(W)$ & 0 & 0 & 0 & 0 \\
\hline NA & King (PS) & 0 & 0 & 0 & 0 \\
\hline NA & Kitsap (PS) & 0 & 0 & 0 & 0 \\
\hline NA & Mason (W) & 0 & 0 & 0 & 0 \\
\hline NA & Pacific (W) & 0 & 0 & 0 & 0 \\
\hline NA & Skamania (SW) & 0 & 0 & 0 & 0 \\
\hline NA & Thurston (PS) & 0 & 0 & 0 & 0 \\
\hline \multirow[t]{2}{*}{ NA } & Wahkiakum (SW) & 0 & 0 & 0 & 0 \\
\hline & State Total & $7,977,901$ & $119,668,519$ & $5,721,360$ & $10,653,630$ \\
\hline
\end{tabular}

1Average based on reports from 1982 through 1989.

2 Incluces residues from both dry and irrigated lands.

3Energy equivalent, 15 million Btu/ton.

Scurce: Washington State Dept. of Agriculture, Washington Agricultural Statistics, 1982-1989. 
Net Barley, Spring Wheat, Winter Wheat Residues by County

Ranked by Net Annual Average (dry tons)

\begin{tabular}{|c|c|c|c|c|c|}
\hline \multirow[b]{2}{*}{ Rank } & \multirow[b]{2}{*}{ County (Region) } & \multicolumn{2}{|c|}{....-Net Annual Average 1} & \multicolumn{2}{|c|}{ 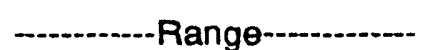 } \\
\hline & & (Dry Tons) $)^{2}$ & (M Btu/Year) $^{3}$ & Minimum & Maximum \\
\hline 1 & Whitman (SE) & 348,616 & $5,229,240$ & 171,686 & 704,802 \\
\hline 2 & Grant (NC) & 281,431 & $4,221,461$ & 210,034 & 419,324 \\
\hline 3 & Adams (SE) & 151,309 & $2,269,629$ & 107,197 & 262,636 \\
\hline 4 & Lincoln (NE) & 149,085 & $2,236,279$ & 33,029 & 330,451 \\
\hline 5 & Walla Walla (SE) & 148,803 & $2,232,043$ & 33,135 & 258,168 \\
\hline 6 & Franklin (NE) & 107,371 & $1,610,558$ & 58,354 & 137,731 \\
\hline 7 & Yakima (SC) & 80,008 & $1,200,126$ & 32,357 & 148,435 \\
\hline 8 & Spokane (NE) & 72,517 & $1,087,753$ & 2,160 & 169,579 \\
\hline 9 & Columbia (SE) & 60,834 & 912,514 & 16,848 & 138,295 \\
\hline 10 & Benton (SE) & 29,737 & 446,053 & 11,009 & 46,262 \\
\hline 11 & Garfield (SE) & 26,581 & 398,711 & 0 & 56,393 \\
\hline 12 & Kittitas (SC) & 14,115 & 211,727 & 4,920 & 23,535 \\
\hline 13 & Skagit (NW) & 9,770 & 146,546 & 1,121 & 21,549 \\
\hline 14 & Klickitat (SC) & 6,372 & 95,584 & 2,674 & 9,320 \\
\hline 15 & Douglas (NC) & 5,408 & 81,118 & 768 & 22,930 \\
\hline 16 & Stevens (NE) & 4,291 & 64,363 & 891 & 12,363 \\
\hline 17 & Ferry (NE) & 1,630 & 24,454 & 0 & 5,427 \\
\hline 18 & Asotin (SE) & 1,627 & 24,401 & 0 & 7,265 \\
\hline 19 & Clark (SW) & 1,432 & 21,478 & 20 & 6,973 \\
\hline 20 & Lewis (SW) & 1,396 & 20,934 & 549 & 2,339 \\
\hline 21 & Snohomish (PS) & 1,032 & 15,476 & 0 & 5,138 \\
\hline 22 & Whatcom (NW) & 979 & 14,683 & 163 & 2,528 \\
\hline 23 & Okanogan (NC) & 931 & 13,971 & 28 & 3,025 \\
\hline 24 & island (NW) & 885 & 13,269 & 535 & 1,398 \\
\hline 25 & Clallam (W) & 62 & 934 & 0 & 461 \\
\hline 26 & Pend Oreille (NE) & 58 & 876 & 0 & 260 \\
\hline 27 & Chelan (NC) & 32 & 486 & 0 & 259 \\
\hline NA & Cowlitz (SW) & 0 & 0 & 0 & 0 \\
\hline NA & Grays Harbor (W) & 0 & 0 & 0 & 0 \\
\hline NA & Jefferson (W) & 0 & 0 & 0 & 0 \\
\hline NA & King (PS) & 0 & 0 & 0 & 0 \\
\hline NA & Kitsap (PS) & 0 & 0 & 0 & 0 \\
\hline NA & Mason (W) & 0 & 0 & 0 & 0 \\
\hline NA & Pacific (W) & 0 & 0 & 0 & 0 \\
\hline NA & Pierce (PS) & 0 & 0 & 0 & 0 \\
\hline NA & San Juan (NW) & 0 & 0 & 0 & 0 \\
\hline NA & Skamania (SW) & 0 & 0 & 0 & 0 \\
\hline NA & Thurston (PS) & 0 & 0 & 0 & 0 \\
\hline \multirow[t]{2}{*}{ NA } & Wahkiakum (SW) & 0 & 0 & 0 & 0 \\
\hline & State Total & $1,506,311$ & $22,594,666$ & 687.478 & $2,796,846$ \\
\hline
\end{tabular}

1 Average based on reports from 1982 through 1989.

2 Includes residues from both dry and irrigated lands.

3Energy equivalent, 15 million Btu/ton.

Source: Washington State Dept. of Agriculture, Washington Agricultural Statistics, 1982-1989. 
Table 1-14

\section{Gross Barley Residues by County}

Ranked by Gross Annual Average (dry tons)

\begin{tabular}{|c|c|c|c|c|c|}
\hline \multirow[b]{2}{*}{ Rank } & \multirow[b]{2}{*}{ County (Region) } & \multicolumn{2}{|c|}{---Gross Annual Average 1} & \multicolumn{2}{|c|}{ 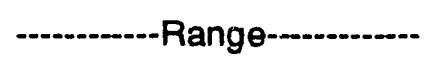 } \\
\hline & & (Dry Tons) $^{2}$ & 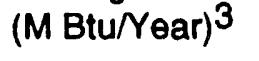 & Minimum & Maximum \\
\hline 1 & Whitman (SE) & 440,617 & $6,609,251$ & 314,940 & 684,600 \\
\hline 2 & Lincoln (NE) & 258,666 & $3,879,984$ & 153,531 & 373,260 \\
\hline 3 & Spokane (NE) & 122,217 & $1,833,261$ & 93,000 & 168,324 \\
\hline 4 & Adams (SE) & 96,772 & $1,451,576$ & 38,919 & 180,540 \\
\hline 5 & Walla Walla (SE) & 85,032 & $1,275,480$ & 45,780 & 131,100 \\
\hline 6 & Garfield (SE) & 72,392 & $1,085,878$ & 54,930 & 106,650 \\
\hline 7 & Grant (NC) & 62,182 & 932,726 & 29,106 & 93,210 \\
\hline 8 & Franklin (SE) & 36,152 & 542,278 & 11,484 & 73,110 \\
\hline 9 & Columbia (SE) & 34,439 & 516,583 & 16,815 & 52,500 \\
\hline 10 & Douglas (NC) & 28,586 & 428,794 & 8,580 & 63,480 \\
\hline 11 & Asotin (SE) & 26,610 & 399,156 & 12,000 & 44,550 \\
\hline 12 & Yakima (SC) & 25,081 & 376,217 & 16,437 & 43.950 \\
\hline 13 & Klickitat (SC) & 20,846 & 312,688 & 9,846 & 34,485 \\
\hline 14 & Benton (SE) & 19,101 & 286,515 & 4,524 & 34,590 \\
\hline 15 & Stevens (NE) & 18,667 & 280,007 & 10,800 & 31,887 \\
\hline 16 & ittitas (SC) & 6,020 & 90,293 & 2,511 & 12,744 \\
\hline 17 & Clark (SW) & 6,007 & 90,101 & 1,440 & 9,384 \\
\hline 18 & Skagit (NW) & 5,599 & 83,987 & 3,510 & 9,555 \\
\hline 19 & Ferry (NE) & 2,179 & 32,681 & 270 & 5,625 \\
\hline 20 & Lewis (SW) & 2,027 & 30,403 & 600 & 4,020 \\
\hline 21 & Whatcom (NW) & 1,633 & 24,497 & 750 & 2,832 \\
\hline 22 & Okanogan (NC) & 1,538 & 23,063 & 690 & 2,769 \\
\hline 23 & Island (NW) & 1,326 & 19,890 & 720 & 2,280 \\
\hline 24 & Snohomish (PS) & 1,049 & 15,728 & 630 & 1,488 \\
\hline 25 & Pend Oreille (NE) & 914 & 13,714 & 600 & 1,950 \\
\hline 26 & Chelan (NC) & 769 & 11,531 & 180 & 2,025 \\
\hline 27 & Clallam (W) & 727 & 10,901 & 0 & 1.710 \\
\hline 28 & San Juan (N) & 544 & 8,156 & 0 & 1,344 \\
\hline NA & Cowlitz (SW) & 0 & 0 & 0 & 0 \\
\hline NA & Grays Harbor (W) & 0 & 0 & 0 & 0 \\
\hline NA & Jefferson $(W)$ & 0 & 0 & 0 & 0 \\
\hline NA & King (PS) & 0 & 0 & 0 & 0 \\
\hline NA & Kitsap (PS) & 0 & 0 & 0 & 0 \\
\hline NA & Mason (W) & 0 & 0 & 0 & 0 \\
\hline NA & Pacific (W) & 0 & 0 & 0 & 0 \\
\hline NA & Pierce (PS) & 0 & 0 & 0 & 0 \\
\hline NA & Skamania (SW) & 0 & 0 & 0 & 0 \\
\hline NA & Thurston (PS) & 0 & 0 & 0 & 0 \\
\hline \multirow[t]{2}{*}{ NA } & Wahkiakum (SW) & 0 & 0 & 0 & 0 \\
\hline & State Total & $1,377,689$ & $20,665,339$ & 832,593 & $2,173,962$ \\
\hline
\end{tabular}

1 Average based on reports from 1982 through 1989.

2 Includes residues from both dry and irrigated lands.

3 Energy equivalent, 15 million Btu/ton.

Source: Washington State Dept. of Agriculture, Washington Agricultural Statistics, 1982-1989. 
Table 1-15

Net Barley Residues by County

Ranked by Net Annual Average (dry tons)

\begin{tabular}{|c|c|c|c|c|c|}
\hline \multirow[b]{2}{*}{ Rank } & \multirow[b]{2}{*}{ County (Region) } & \multicolumn{2}{|c|}{.....Net Anni al Average1..... } & \multicolumn{2}{|c|}{ 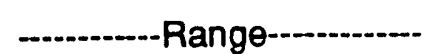 } \\
\hline & & (Dry Tons) ${ }^{2}$ & $(\mathrm{M} \mathrm{Btu} / \text { Year })^{3}$ & Minimum & Maximum \\
\hline 1 & Grant (NC) & 15,115 & 226,723 & 5,006 & 25,100 \\
\hline 2 & Lincoln (NE) & 6,570 & 98,556 & 1,649 & 13,944 \\
\hline 3 & Yakima (SC) & 5,470 & 82,054 & 3,373 & 9,152 \\
\hline 4 & Franklin (SE) & 3,966 & 59,494 & 2,000 & 6,460 \\
\hline 5 & Adams (SE) & 3,765 & 56,468 & 0 & 8,090 \\
\hline 6 & Benton (SE) & 2,442 & 36,623 & 420 & 5,930 \\
\hline 7 & Walla Walla (SE) & 2,421 & 36,311 & 0 & 7,590 \\
\hline 8 & Kittitas (SC) & 1,798 & 26,966 & 580 & 3,420 \\
\hline 9 & Spokane (NE) & 1,642 & 24,632 & 140 & 3,584 \\
\hline 10 & Skagit (NW) & 537 & 8,049 & 260 & 805 \\
\hline 11 & Klickitat (SC) & 395 & 5,927 & 0 & 1,218 \\
\hline 12 & Stevens (NE) & 360 & 5,393 & 0 & 1,251 \\
\hline 13 & Whitman (SE) & 287 & 4,307 & 0 & 1,110 \\
\hline 14 & Ferry (NE) & 182 & 2,728 & 0 & 805 \\
\hline 15 & Douglas (NC) & 91 & 1,369 & 0 & 280 \\
\hline 16 & Pend Oreille (NE) & 55 & 829 & 0 & 260 \\
\hline 17 & Garfield (SE) & 36 & 544 & 0 & 110 \\
\hline 18 & Island (NW) & 36 & 540 & 0 & 200 \\
\hline 19 & Clallam (W) & 26 & 394 & 0 & 210 \\
\hline 20 & Snohomish (PS) & 16 & 244 & 0 & 130 \\
\hline 21 & Asotin (SE) & 9 & 131 & 0 & 70 \\
\hline 22 & Clark (SW) & 8 & 113 & 0 & 50 \\
\hline 23 & Okanogan (NC) & 7 & 103 & 0 & 55 \\
\hline 24 & Columbia (SE) & 4 & 56 & 0 & 30 \\
\hline NA & Chelan (NC) & 0 & 0 & 0 & 0 \\
\hline NA & Cowlitz (SW) & 0 & 0 & 0 & 0 \\
\hline NA & Grays Harbor (W) & 0 & 0 & 0 & 0 \\
\hline NA & Jefterson $(W)$ & 0 & 0 & 0 & 0 \\
\hline NA & King (PS) & 0 & 0 & 0 & 0 \\
\hline NA & Kitsap (PS) & 0 & 0 & 0 & 0 \\
\hline NA & Lewis (SW) & 0 & 0 & 0 & 0 \\
\hline NA & Mason (W) & 0 & 0 & 0 & 0 \\
\hline NA & Pacific (W) & 0 & 0 & 0 & 0 \\
\hline NA & Pierce (PS) & 0 & 0 & 0 & 0 \\
\hline NA & San Juan (NW) & 0 & 0 & 0 & 0 \\
\hline NA & Skamania (SW) & 0 & 0 & 0 & 0 \\
\hline NA & Thurston (PS) & 0 & 0 & 0 & 0 \\
\hline NA & Wahkiakum (SW) & 0 & 0 & 0 & 0 \\
\hline \multirow[t]{2}{*}{ NA } & Whatcom (NW) & 0 & 0 & 0 & 0 \\
\hline & State Total & 45,237 & 678,551 & 13,428 & 89,854 \\
\hline
\end{tabular}

1 Average based on reports from 1982 through 1989.

2 Includes residues from both dry and irrigated lands.

3Energy equivalent, 15 million Btu/ton.

Source: Washington State Dept. of Agriculture, Washington Agricultural Statistics, 1982-1989. 
Table 1-16

\section{Gross Spring Wheat Residues by County}

Ranked by Gross Annual Average (dry tons)

\begin{tabular}{|c|c|c|c|c|c|}
\hline \multirow[b]{2}{*}{ Rank } & \multirow[b]{2}{*}{ County (Region) } & \multicolumn{2}{|c|}{-.--Gross Annual Average ${ }^{1} \ldots$} & \multicolumn{2}{|c|}{ 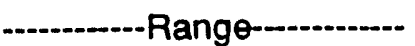 } \\
\hline & & (Dry Tons) $)^{2}$ & (M Btu/Year) $^{3}$ & Minimum & Maximum \\
\hline 1 & Grant (NC) & 113,602 & $1,704,032$ & 88,491 & 159,705 \\
\hline 2 & Adams (SE) & 104,003 & $1,560,049$ & 51,636 & 326,820 \\
\hline 3 & I.incoln (NE) & 86,912 & $1,303,673$ & 17,004 & 347,100 \\
\hline 4 & Whitman (SE) & 72,482 & $1,087,223$ & 6,981 & 333,645 \\
\hline 5 & Yakima (SC) & 46,717 & 700,757 & 20,085 & 113,529 \\
\hline 6 & Benton (SE) & 45,123 & 676,845 & 10,335 & 81,471 \\
\hline 7 & Franklin (SE) & 32,609 & 489,133 & 11,622 & 85,995 \\
\hline 8 & Douglas (NC) & 26,808 & 402,114 & 10,920 & 42,978 \\
\hline 9 & Spokane (NE) & 24,185 & 362,773 & 11,661 & 83,304 \\
\hline 10 & Walla Walla (SE) & 22,308 & 334,620 & 8,697 & 58,500 \\
\hline 11 & Klickitat (SC) & 10,525 & 157,877 & 2,691 & 20,475 \\
\hline 12 & Kittitas (SC) & 9,589 & 143,837 & 7,956 & 11,739 \\
\hline 13 & Stevens (NE) & 7,800 & 117,000 & 4,329 & 14,313 \\
\hline 14 & Gartield (SE) & 7,122 & 106,836 & 3,861 & 13,143 \\
\hline 15 & Columbia (SE) & 6,684 & 100,254 & 3,744 & 13,689 \\
\hline 16 & Okanogan (NC) & 4,748 & 71,224 & 1,131 & 6,942 \\
\hline 17 & Snohomish (PS) & 4,149 & 62,229 & 0 & 15,600 \\
\hline 18 & Asotin (SE) & 2,954 & 44,314 & 1,092 & 5,304 \\
\hline 19 & Ferry (NE) & 1,550 & 23,254 & 507 & 3,081 \\
\hline 20 & Chelan (NC) & 1,365 & 20,475 & 702 & 1,950 \\
\hline 21 & Skagit (NW) & 1,087 & 16,307 & 0 & 3,081 \\
\hline 22 & Clallam (W) & 692 & 10,384 & 0 & 4,251 \\
\hline 23 & Lewis (SW) & 327 & 4,899 & 0 & 1,560 \\
\hline 24 & Pend Oreille (NE) & 185 & 2,779 & 0 & 312 \\
\hline 25 & Pierce (PS) & 88 & 1,316 & 0 & 702 \\
\hline NA & Clark (SW) & 0 & 0 & 0 & 0 \\
\hline NA & Cowlitz (SW) & 0 & 0 & 0 & 0 \\
\hline NA & Grays Harbor (W) & 0 & 0 & 0 & 0 \\
\hline NA & Island (NW) & 0 & 0 & 0 & 0 \\
\hline NA & Jefferson $(W)$ & 0 & 0 & 0 & 0 \\
\hline NA & King (PS) & 0 & 0 & 0 & 0 \\
\hline NA & Kitsap (PS) & 0 & 0 & 0 & 0 \\
\hline NA & Mason (W) & 0 & 0 & 0 & 0 \\
\hline NA & Pacific (W) & 0 & 0 & 0 & 0 \\
\hline NA & San Juan (NW) & 0 & 0 & 0 & 0 \\
\hline NA & Skamania (SW) & 0 & 0 & 0 & 0 \\
\hline NA & Thurston (PS) & 0 & 0 & 0 & 0 \\
\hline NA & Wahkiakum (SW) & 0 & 0 & 0 & 0 \\
\hline \multirow[t]{2}{*}{ NA } & Whatcom (NW) & 0 & 0 & 0 & 0 \\
\hline & State Total & 633,614 & $9,504,203$ & 263,445 & $1,749,189$ \\
\hline
\end{tabular}

1 Average based on reports from 1982 through 1989.

2 includes residues from both dry and irrigated lands.

3Energy equivalent, 15 million Btu/ton.

Source: Washington State Dept. of Agriculture, Washington Agricultural Statistics, 1982-1989. 
Table 1-17

Net Spring Wheat Residues by County

Ranked by Net Annual Average (dry tons)

\begin{tabular}{|c|c|c|c|c|c|}
\hline \multirow[b]{2}{*}{ Rank } & \multirow[b]{2}{*}{ County (Region) } & \multicolumn{2}{|c|}{...--Net Annual Average 1 -..... } & \multicolumn{2}{|c|}{ 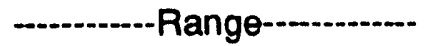 } \\
\hline & & (Dry Tons) $^{2}$ & (M Btu/Year) ${ }^{3}$ & Minimum & Maximum \\
\hline 1 & Grant (NC) & 38,976 & 584,634 & $32,22.3$ & 52,600 \\
\hline 2 & Adams (SE) & 22,474 & 337,114 & 9,553 & 74,046 \\
\hline 3 & Yakima (SC) & 16,980 & 254,693 & 7,658 & 37,599 \\
\hline 4 & Franklin (SE) & 11,464 & 171,964 & 3,876 & 28,584 \\
\hline 5 & Benton (SE) & 6,752 & 101,286 & 393 & 14,515 \\
\hline 6 & Lincoln (NE) & 6,539 & 98,078 & 0 & 17,720 \\
\hline 7 & Kittitas (SC) & 3,278 & 49,163 & 2,020 & 5,027 \\
\hline 8 & Klickitat (SC) & 2,007 & 30,107 & 287 & 5,728 \\
\hline 9 & Spokane (NE) & 1,246 & 18,692 & 4 & 2,590 \\
\hline 10 & Walla Walla (SE) & 1,023 & 15,345 & 184 & 2,856 \\
\hline 11 & Douglas (NC) & 413 & 6,195 & 0 & 1,605 \\
\hline 12 & Whitman (SE) & 194 & 2,903 & 0 & 872 \\
\hline 13 & Stevens (NE) & 171 & 2,571 & 0 & 540 \\
\hline 14 & Okanogan (NC) & 137 & 2,053 & 0 & 560 \\
\hline 15 & Ferry (NE) & 108 & 1,613 & 0 & 389 \\
\hline 16 & Skagit (NW) & 77 & 1,159 & 0 & 331 \\
\hline 17 & Snohomish (PS) & 73 & 1,099 & 0 & 393 \\
\hline 18 & Clallam $(W)$ & 36 & 540 & 0 & 251 \\
\hline NA & Asotin (SE) & 0 & 0 & 0 & 0 \\
\hline NA & Chelan (NC) & 0 & 0 & 0 & 0 \\
\hline NA & Clark (SW) & 0 & 0 & 0 & 0 \\
\hline NA & Columbia (SE) & 0 & 0 & 0 & 0 \\
\hline NA & Cowlitz (SW) & 0 & 0 & 0 & 0 \\
\hline NA & Garfield (SE) & 0 & 0 & 0 & 0 \\
\hline NA & Grays Harbor (W) & 0 & 0 & 0 & 0 \\
\hline NA & Island (NW) & 0 & 0 & 0 & 0 \\
\hline NA & Jefferson $(W)$ & 0 & 0 & 0 & 0 \\
\hline NA & King (PS) & 0 & 0 & 0 & 0 \\
\hline NA & Kitsap (PS) & 0 & 0 & 0 & 0 \\
\hline NA & Lewis (SW) & 0 & 0 & 0 & 0 \\
\hline NA & Mason (W) & 0 & 0 & 0 & 0 \\
\hline NA & Pacific (W) & 0 & 0 & 0 & 0 \\
\hline NA & Pend Oreille (NE) & 0 & 0 & 0 & 0 \\
\hline NA & Pierce (PS) & 0 & 0 & 0 & 0 \\
\hline NA & San Juan (NW) & 0 & 0 & 0 & 0 \\
\hline NA & Skamania (SW) & 0 & 0 & 0 & 0 \\
\hline NA & Thurston (PS) & 0 & 0 & 0 & 0 \\
\hline NA & Wahkiakum (SW) & 0 & 0 & 0 & 0 \\
\hline \multirow[t]{2}{*}{ NA } & Whatcom (NW) & 0 & 0 & 0 & 0 \\
\hline & State Total & 111,947 & $1,679,205$ & 56,198 & 246,206 \\
\hline
\end{tabular}

Source: Washington State Dept. of Agriculture, Washington Agricultural Statistics, 1982-1989. 
Table 1-18

Gross Winter Wheat Residues by County

Ranked by Gross Annual Average (dry tons)

\begin{tabular}{|c|c|c|c|c|c|}
\hline \multirow[b]{2}{*}{ Rank } & \multirow{2}{*}{ County (Region) } & \multicolumn{2}{|c|}{...-Gross Annual Average ${ }^{1}$} & \multicolumn{2}{|c|}{-Range-- } \\
\hline & & (Dry Tons) $^{2}$ & (M Btu/Year) ${ }^{3}$ & Minimum & Maximum \\
\hline 1 & Whitman (SE) & $1,202,217$ & $18,033,249$ & 772,650 & $1,712,988$ \\
\hline 2 & Lincoln (NE) & 782,238 & $11,733,570$ & 256,785 & $1,034,382$ \\
\hline 3 & Adams (SE) & 722,280 & $10,834,207$ & 286,110 & $1,094,664$ \\
\hline 4 & Walla Walla (SE) & 635,556 & $9,533,334$ & 457,215 & 807,942 \\
\hline 5 & Grant (NC) & 575,227 & $8,628,406$ & 357,765 & 813,501 \\
\hline 6 & Douglas (NC) & 401,604 & $6,024,065$ & 181,560 & 569,058 \\
\hline 7 & Spokane (NE) & 327,796 & $4,916,942$ & 234,549 & 474,555 \\
\hline 8 & Franklin (SE) & 302,581 & $4,538,712$ & 149,838 & 450,789 \\
\hline 9 & Columbia (SE) & 264,524 & $3,967,864$ & 199,920 & 362,865 \\
\hline 10 & Benton (SE) & 192,136 & $2,882,042$ & 109,191 & 310,794 \\
\hline 11 & Gartield (SE) & 179,348 & $2,690,218$ & 125,307 & 217,107 \\
\hline 12 & Yakima (SC) & 136,444 & $2,046,662$ & 58,497 & 274,074 \\
\hline 13 & Klickitat (SC) & 82,123 & $1,231,841$ & 64,923 & 112,863 \\
\hline 14 & Asotin (SE) & 58,319 & 874,778 & 34,272 & 77,265 \\
\hline 15 & Stevens (NE) & 23,396 & 350,944 & 15,147 & 32,181 \\
\hline 16 & Skagit (NW) & 20,062 & 300,932 & 1,530 & 40,494 \\
\hline 17 & Kittitas (SC) & 19,259 & 288,883 & 3,213 & 34,272 \\
\hline 18 & Okanogan (NC) & 15,574 & 233,612 & 8,415 & 25,092 \\
\hline 19 & Clark (SW) & 5,706 & 85,584 & 1,020 & 16,473 \\
\hline 20 & Lewis (SW) & 4,239 & 63,591 & 1,989 & 7,140 \\
\hline 21 & Ferry (NE) & 3,831 & 57,471 & 459 & 9,333 \\
\hline 22 & Chelan (NC) & 3,678 & 55,176 & 1,989 & 6,579 \\
\hline 23 & Whatcom (NW) & 3,385 & 50,777 & 663 & 6,528 \\
\hline 24 & Island (NW) & 2,161 & 32,417 & 1,632 & 3,060 \\
\hline 25 & Snohomish (PS) & 2,091 & 31,365 & 0 & 12,138 \\
\hline 26 & Pend Oreille (NE) & 440 & 6,598 & 0 & 1,275 \\
\hline NA & Clallam (W) & 0 & 0 & 0 & 0 \\
\hline NA & Cowlitz (SW) & 0 & 0 & 0 & 0 \\
\hline NA & Grays Harbor (W) & 0 & 0 & 0 & 0 \\
\hline NA & Jefferson (W) & 0 & 0 & 0 & 0 \\
\hline NA & King (PS) & 0 & 0 & 0 & 0 \\
\hline NA & Kitsap (PS) & 0 & 0 & 0 & 0 \\
\hline NA & Mason (W) & 0 & 0 & 0 & 0 \\
\hline NA & Pacific (W) & 0 & 0 & 0 & 0 \\
\hline NA & Pierce (PS) & 0 & 0 & 0 & 0 \\
\hline NA & San Juan (NW) & 0 & 0 & 0 & 0 \\
\hline NA & St amania (SW) & 0 & 0 & 0 & 0 \\
\hline NA & Thurston (PS) & 0 & 0 & 0 & 0 \\
\hline NA & Wahkiakum (SW) & 0 & 0 & 0 & 0 \\
\hline & State Total & $5,966,216$ & $89,493,240$ & $3,324,639$ & $8,507,412$ \\
\hline
\end{tabular}

1 Average based on reports from 1982 through 1989.

2 Includes residues from both dry and irrigated lands.

${ }^{3}$ Energy equivalent, 15 million Btu/ton.

Source: Washington State Dept. of Agriculture, Washington Agricultural Statistics, 1982-1989. 


\begin{tabular}{|c|c|c|c|c|c|}
\hline \multirow[b]{2}{*}{ Rank } & \multirow[b]{2}{*}{ County (Region) } & \multicolumn{2}{|c|}{....-Net Annual Average 1 ....... } & \multicolumn{2}{|c|}{ 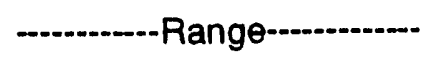 } \\
\hline & & (Dry Tons) $^{2}$ & (M Btu/Year) $^{3}$ & Minimum & Maximum \\
\hline 1 & Whitman (SE) & 348,135 & $5,222,031$ & 171,686 & 704,388 \\
\hline 2 & Grant (NC) & 227,340 & $3,410,103$ & 149,181 & 362,001 \\
\hline 3 & Walla Walla (SE) & 145,359 & $2,180,387$ & 28,720 & 249,936 \\
\hline 4 & Lincoln (NE) & 135,976 & $2,039,646$ & 13,660 & 316,360 \\
\hline 5 & Adams (SE) & 125,070 & $1,876,048$ & 68.666 & 244,814 \\
\hline 6 & Franklin (SE) & 91,940 & $1,379,100$ & 45,184 & 122,889 \\
\hline 7 & Spokane (NE) & 69,629 & $1,044,429$ & 1,409 & 163,405 \\
\hline 8 & Columbia (SE) & 60,831 & 912,458 & 16,848 & 138,265 \\
\hline 9 & Yakima (SC) & 57,559 & 863,379 & 15,347 & 134,988 \\
\hline 10 & Garfield (SE) & 26,545 & 398,168 & 0 & 56,393 \\
\hline 11 & Benton (SE) & 20,543 & 308,145 & 3,814 & 43,540 \\
\hline 12 & Skagit (NW) & 9,156 & 137,336 & 280 & 20,744 \\
\hline 13 & Kittitas (SC) & 9,040 & 135,598 & 828 & 17,045 \\
\hline 14 & Douglas (NC) & 4,904 & 73,554 & 208 & 21,255 \\
\hline 15 & Klickitat (SC) & 3,970 & 59,550 & 730 & 7,300 \\
\hline 16 & Stevens (NE) & 3,760 & 56,400 & 891 & 11,931 \\
\hline 17 & Asotin (SE) & 1,618 & 24,270 & 0 & 7,265 \\
\hline 18 & Clark (SW) & 1,424 & 21,366 & 20 & 6,973 \\
\hline 19 & Lewis (SW) & 1,396 & 20,934 & 549 & 2,339 \\
\hline 20 & Ferry (NE) & 1,341 & 20.113 & 0 & 4,233 \\
\hline 21 & Whatcom (NW) & 979 & 14,683 & 163 & 2,528 \\
\hline 22 & Snohomish (PS) & 872 & 13,084 & 0 & 5,138 \\
\hline 23 & Island (NW) & 849 & 12,729 & 535 & 1,310 \\
\hline 24 & Okanogan (NC) & 788 & 11,815 & 4 & 2,845 \\
\hline 25 & Chelan (NC) & 32 & 486 & 0 & 259 \\
\hline 26 & Pend Oreille (NE) & 3 & 47 & 0 & 25 \\
\hline NA & Clallam (W) & 0 & 0 & 0 & 0 \\
\hline NA & Cowlitz (SW) & 0 & 0 & 0 & 0 \\
\hline NA & Grays Harbor (W) & 0 & 0 & 0 & 0 \\
\hline NA & Jefferson $(W)$ & 0 & 0 & 0 & 0 \\
\hline NA & King (PS) & 0 & 0 & 0 & 0 \\
\hline NA & Kitsap (PS) & 0 & 0 & 0 & 0 \\
\hline NA & Mason (W) & 0 & 0 & 0 & 0 \\
\hline NA & Pacific (W) & 0 & 0 & 0 & 0 \\
\hline NA & Pierce (PS) & 0 & 0 & 0 & 0 \\
\hline NA & San Juan (NW) & 0 & 0 & 0 & 0 \\
\hline NA & Skamania (SW) & 0 & 0 & 0 & 0 \\
\hline NA & Thurston (PS) & 0 & 0 & 0 & 0 \\
\hline NA & Wahkiakum (SW) & 0 & 0 & 0 & 0 \\
\hline & State Total & $1,349,057$ & $20,235,860$ & 518,723 & $2,648,169$ \\
\hline
\end{tabular}

1 Average based on reports from 1982 through 1989.

2 Includes residues from both dry and irrigated lands.

3 Energy equivalent, 15 inillion Btu/ton.

Source: Washington State Dept. of Agriculture, Washington Agricultural Statistics, 1982-1989. 
Table $1-20$

Costs of Procuring Agricultural Field Residues

Cost Component

$\$$ Tron

\$MBtu

Collection (fuel, labor, capital)

Fertilizer Value

Profit to Collector

Local Storage

Transportation up to $\mathbf{4 0}$ Miles

0.33

0.27

0.66

Total

33

2.20

Source: U.S. Department of Energy, "Biomass Energy Project Development Guidebook," 1989. 


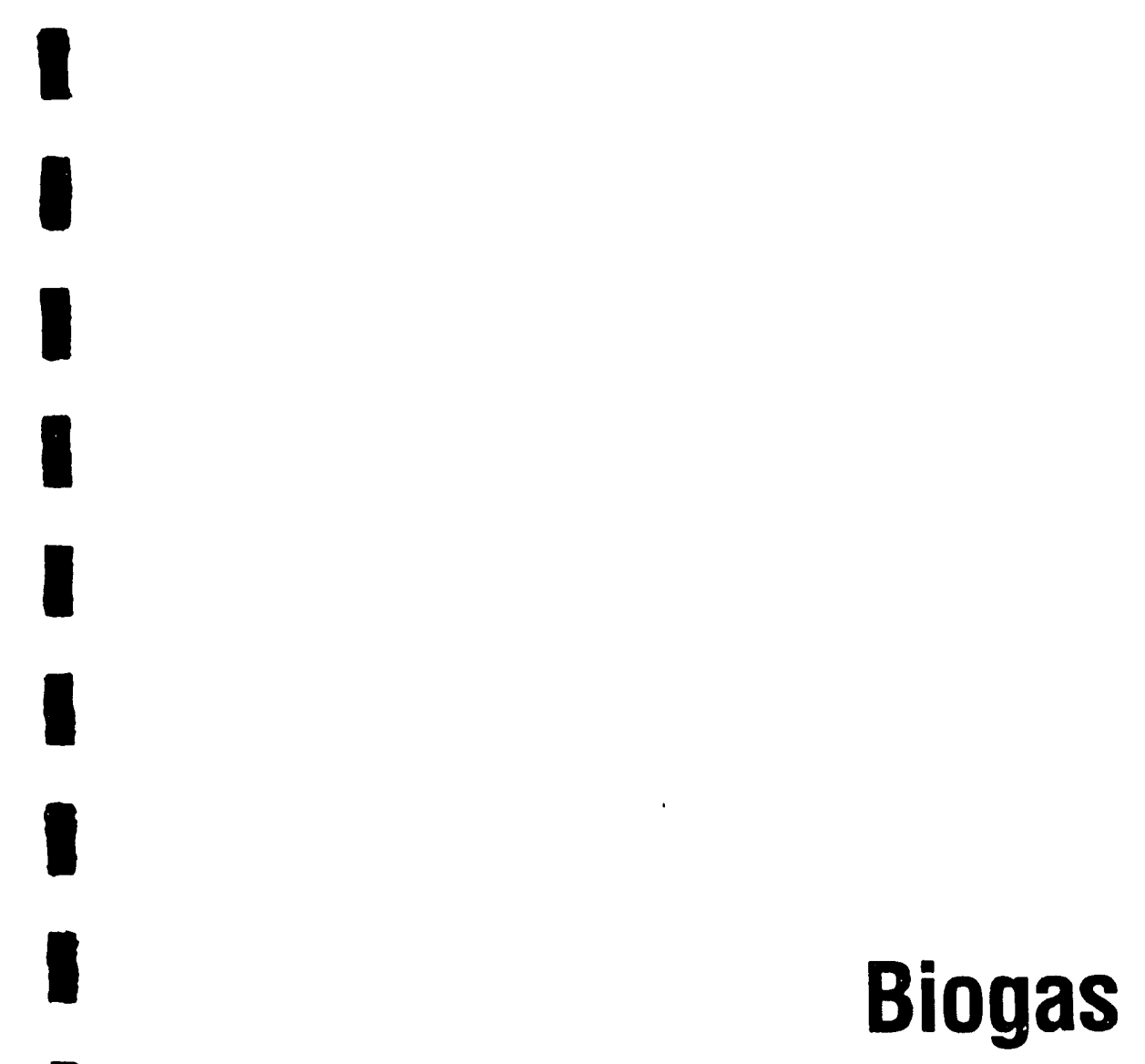

Map and Tables

1

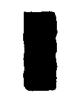

0

I

I

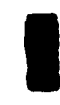


Map 1-9

\section{Biogas Output}

Bugas Output

Million Cubic feet

By Region
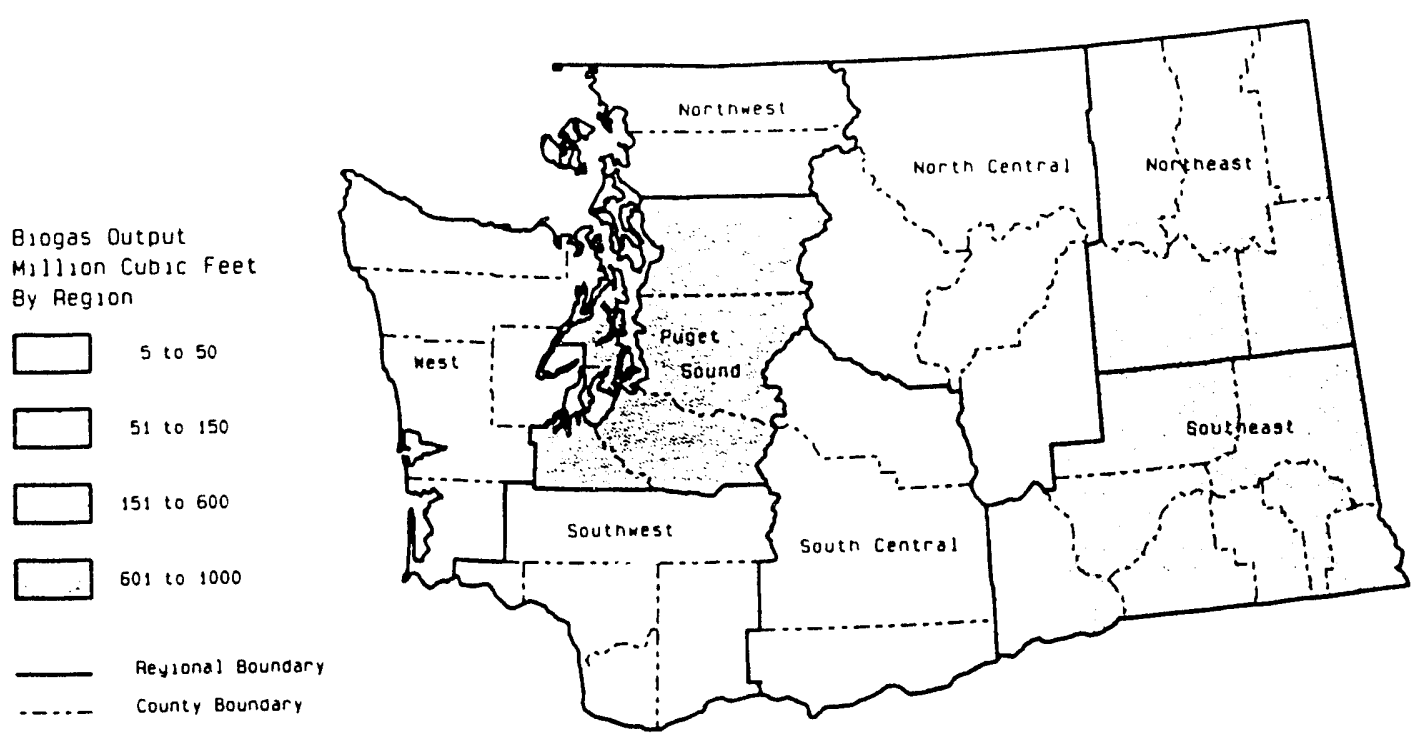

State of Wushington 
Table 1-21

\section{Biogas Generated by Region}

Sorted by Biogas Output $\left(\mathrm{MF}^{3} \mathrm{Y}\right)$

\begin{tabular}{rlrr}
\hline Rank & Region & $\begin{array}{r}\text { Biogas } \\
\text { Output } \\
\left(\mathrm{MF}^{3} / \mathrm{Y}\right)\end{array}$ & $\begin{array}{r}\text { Energy } \\
\text { Equivalent } \\
\text { (MBtu) }\end{array}$ \\
\hline 1 & Puget Sound & 956 & 573,600 \\
2 & Northeast & 230 & 138,000 \\
3 & Southeast & 70 & 42,000 \\
4 & North Central & 24 & 14,400 \\
5 & South Central & 24 & 14,400 \\
6 & Southwest & 21 & 12,600 \\
7 & Northwest & 20 & 12,000 \\
8 & West & 5.5 & 3,300 \\
& State Total & 1,351 & 810,300
\end{tabular}

Source: Washington State Energy Office. 1990 Directory of Biomass Energy Facilities. 1990 
Table 1-22

\section{Biogas Generated by County}

Sorted by Biogas Output $\left(M^{3} / N\right.$ )

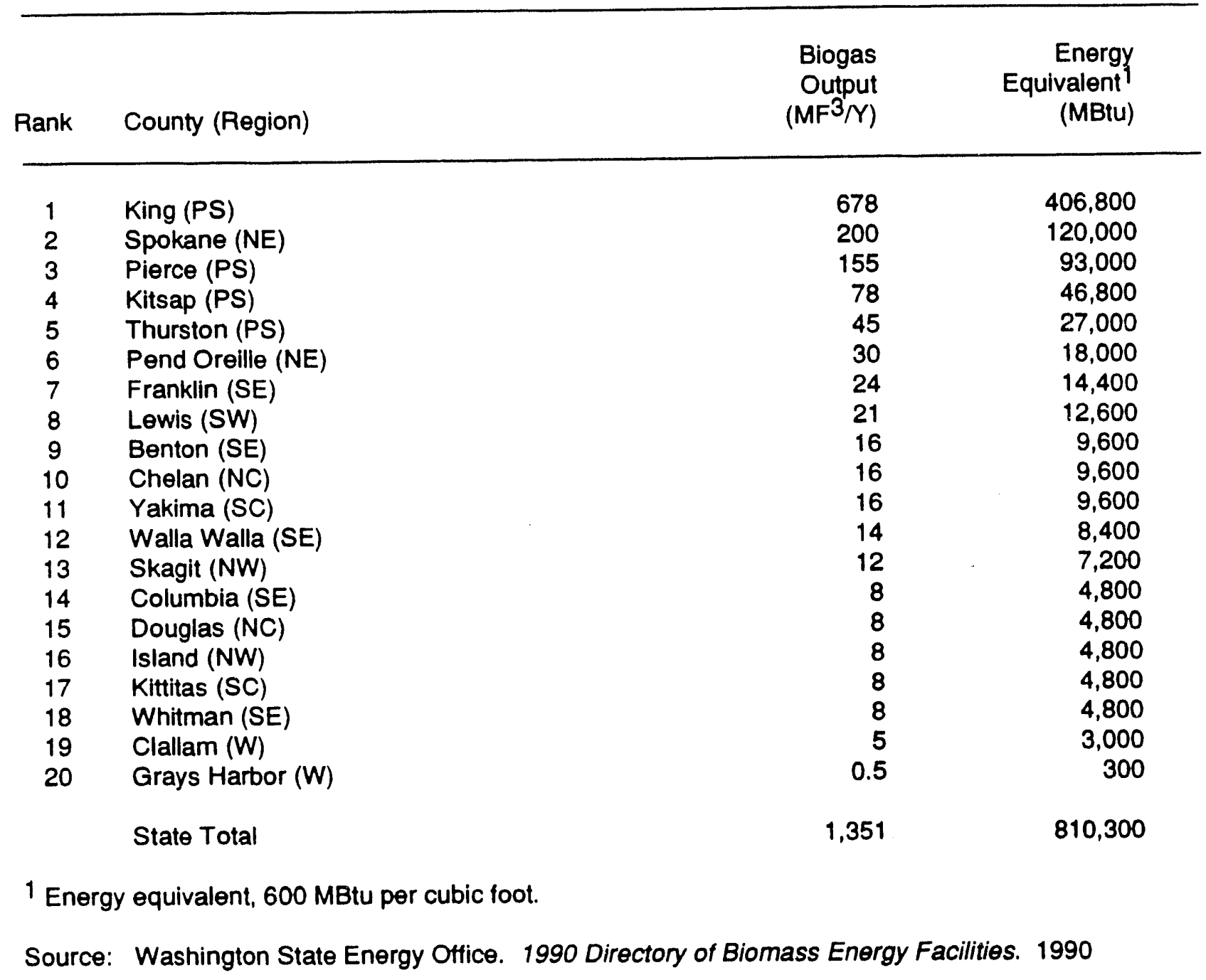




\section{Municipal Solid Waste}

Map and Tables 
Map 1-10

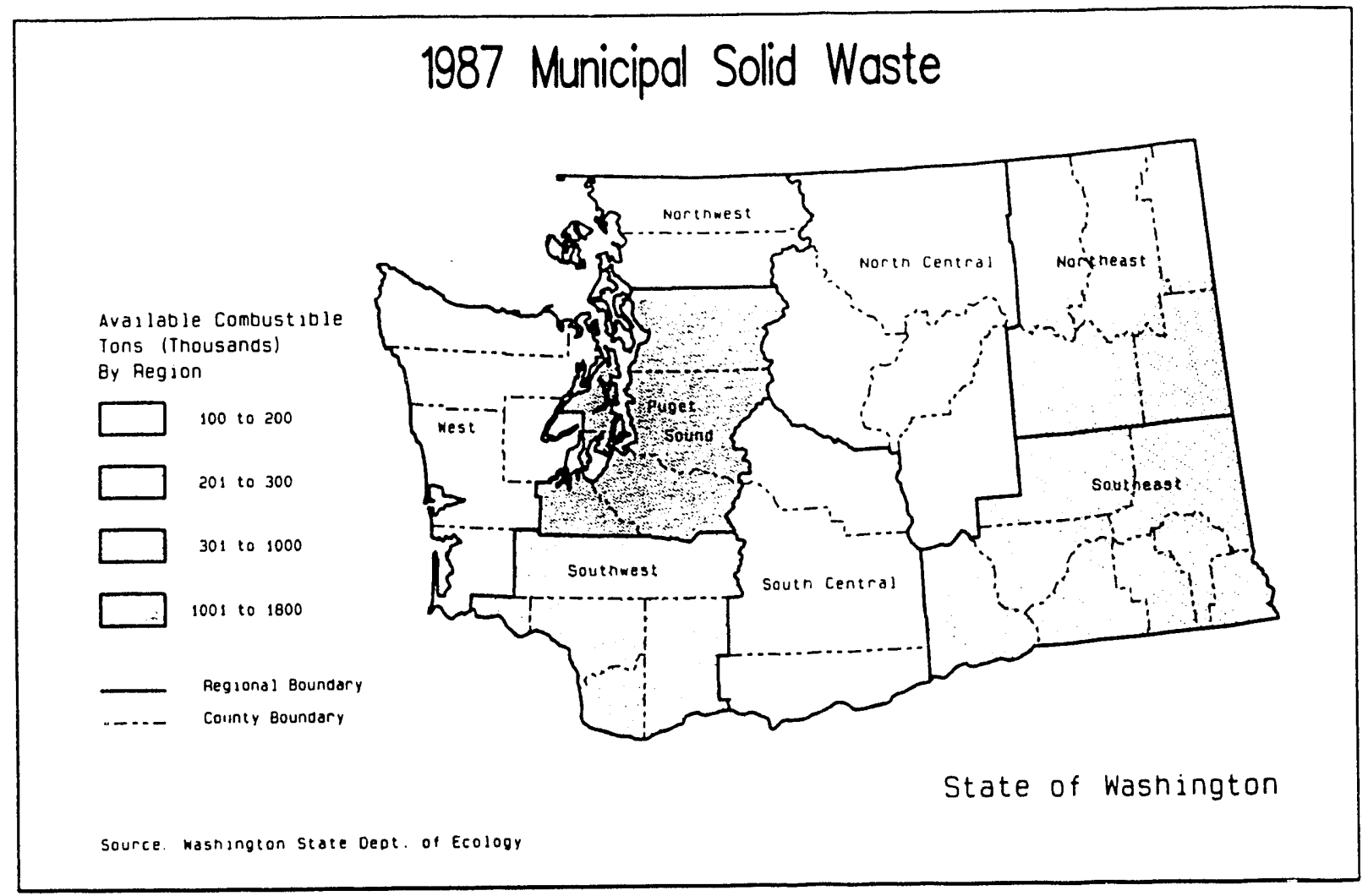


Table 1-23

\section{Combustible Fraction of Municipal Solid Waste}

Disposed in Washington State By Region

\begin{tabular}{rlrr}
\hline Rank & Region & $\begin{array}{r}\text { Combustible } \\
\text { Waste } \\
\text { (Tons) }\end{array}$ & $\begin{array}{r}\text { Energy } \\
\text { Equivalent } \\
\text { (M Btu) }\end{array}$ \\
\hline 1 & Puget Sound & $1,766,246$ & $22,961,198$ \\
2 & Northeast & 306,426 & $3,983,538$ \\
3 & Southeast & 222,574 & $2,893,462$ \\
4 & Southwest & 203,434 & $2,644,642$ \\
5 & West & 166,345 & $2,162,485$ \\
6 & Northwest & 158,679 & $2,062,827$ \\
7 & North Central & 151,087 & $1,964,131$ \\
8 & South Central & 148,281 & $1,927,653$ \\
& Total & $3,123,072$ & $40,599,936$ \\
1 & & &
\end{tabular}

Source: Washington State Department of Ecology, Best Management Practices Analysis for Soild Waste 1987 Recycling and Waste Stream Survey, 1988 
Table 1-24

\section{Estimated Waste Generated in 1989 by County}

Ranked by Estimated Annual Tons

\begin{tabular}{|c|c|c|c|}
\hline Rank & County & $\begin{array}{r}1989 \\
\text { Population }\end{array}$ & $\begin{array}{r}\text { Estimated } \\
\text { Annual } 1 \\
\text { (Tons) }\end{array}$ \\
\hline 1 & King & $1,446,000$ & $1,651,983$ \\
\hline 2 & Pierce & 560,900 & 640,800 \\
\hline 3 & Snohomish & 430,400 & 491,710 \\
\hline 4 & Spokane & 358,000 & 408,997 \\
\hline 5 & Clark & 220,400 & 251,796 \\
\hline 6 & Yakima & 187,800 & 214,552 \\
\hline 7 & Kitsap & 181,500 & 207,355 \\
\hline 8 & Thurston & 155,100 & 177,194 \\
\hline 9 & Whatcom & 122,200 & 139,607 \\
\hline 10 & Benton & 104,100 & 118,929 \\
\hline 11 & Cowlitz & 82,100 & 93,795 \\
\hline 12 & Skagit & 72,400 & 82,713 \\
\hline 13 & Grays Harbor & 63,600 & 72,660 \\
\hline 14 & Lewis & 58,000 & 66,262 \\
\hline 15 & Island & 55,300 & 63,177 \\
\hline 16 & Clallam & 55,200 & 63,063 \\
\hline 17 & Grant & 51,900 & 59,293 \\
\hline 18 & Walla Walla & 48,800 & 55,752 \\
\hline 19 & Chelan & 48,600 & 55,523 \\
\hline 20 & Whitman & 37,600 & 42,956 \\
\hline 21 & Mason & 37,500 & 42,842 \\
\hline 22 & Franklin & 34,200 & 39,072 \\
\hline 23 & Okanogan & 31,700 & 36,216 \\
\hline 24 & Stevens & 30,500 & 34,845 \\
\hline 25 & Douglas & 25,400 & 29,018 \\
\hline 26 & Kittitas & 25,400 & 29,018 \\
\hline 27 & Jefferson & 19,200 & 21,935 \\
\hline 28 & Pacific & 17,700 & 20,221 \\
\hline 29 & Asotin & 17,600 & 20,107 \\
\hline 30 & Klickitat & 16,700 & 19,079 \\
\hline 31 & Adams & 13,400 & 15,309 \\
\hline 32 & San Juan & 9,700 & 11,082 \\
\hline 33 & Pend Oreille & 8,900 & 10,168 \\
\hline 34 & Lincoln & 8,800 & 10,054 \\
\hline 35 & Skamania & 8,100 & 9,254 \\
\hline 36 & Ferry & 6,100 & 6,969 \\
\hline 37 & Columbia & 4,100 & 4,684 \\
\hline 38 & Wahkiakum & 3,500 & 3,999 \\
\hline \multirow[t]{2}{*}{39} & Gartield & 2,300 & 2,628 \\
\hline & State Total & $4,660,700$ & $5,324,617$ \\
\hline
\end{tabular}

${ }^{1}$ Based on Washington State Dept. of Ecology estimates of 6.26 pounds per person per day; $6.26 \mathrm{lbs} /$ day $\times 365$ days/yr $\times$ county population.

Source: Washington State Dept. of Ecology 


\section{Forest Residues}

Figures, Maps, and Tables 
Figure l-5

\section{Percent of Logging Residues Generated by Land Ownership}

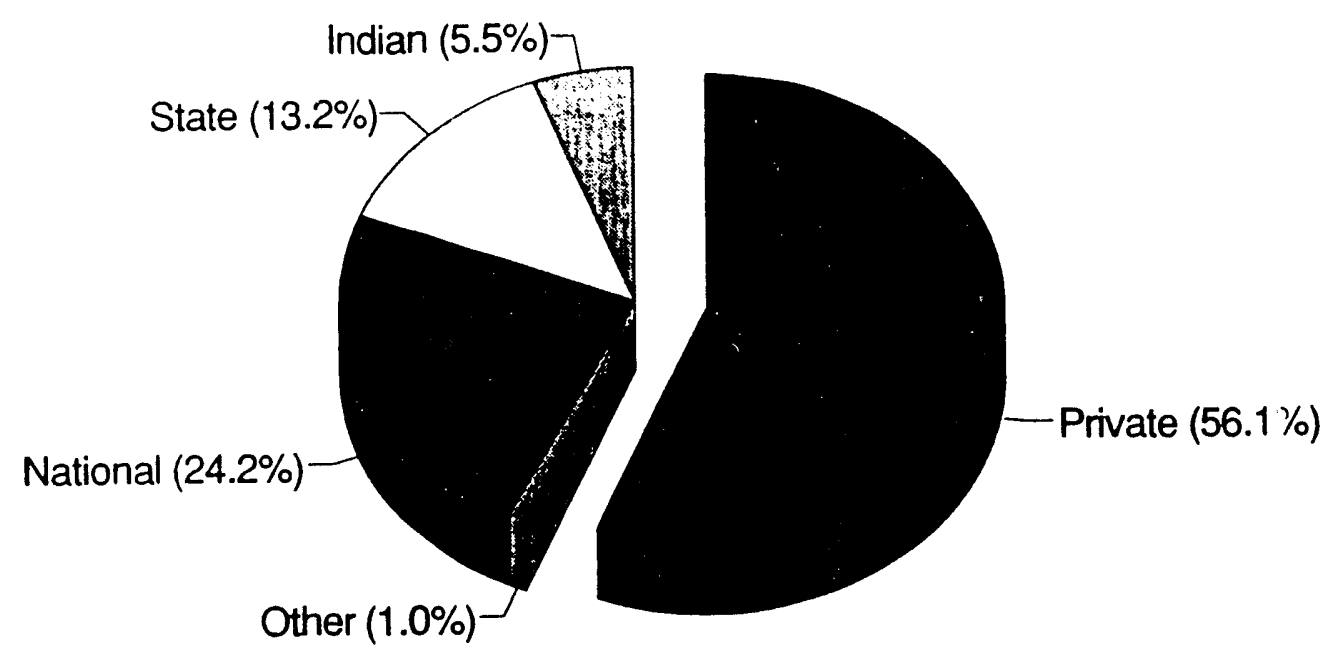

Figure l-6

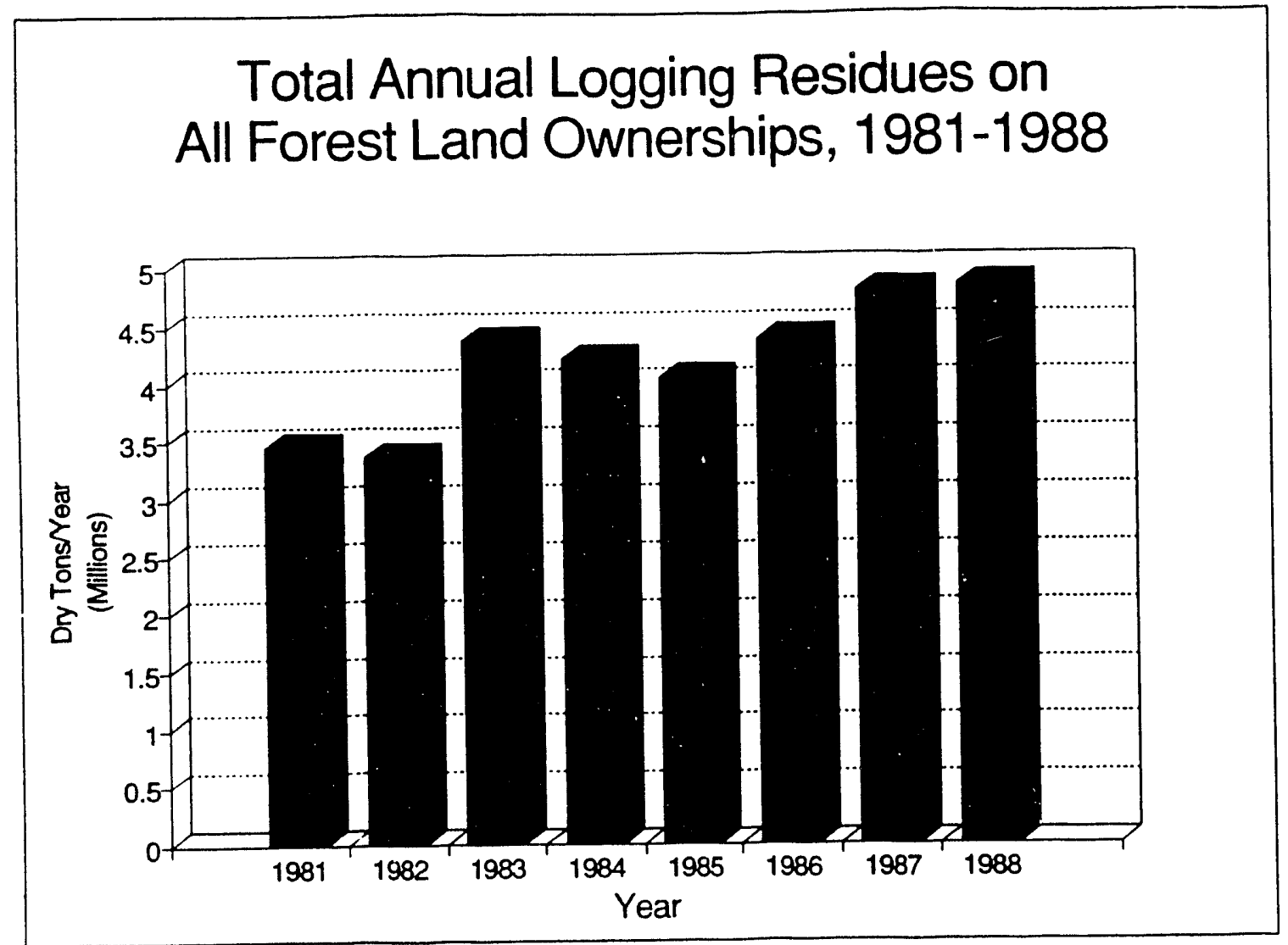


Figure 1-7

Total Annual Logging Residues on

Private Forest Lands, 1981-1988

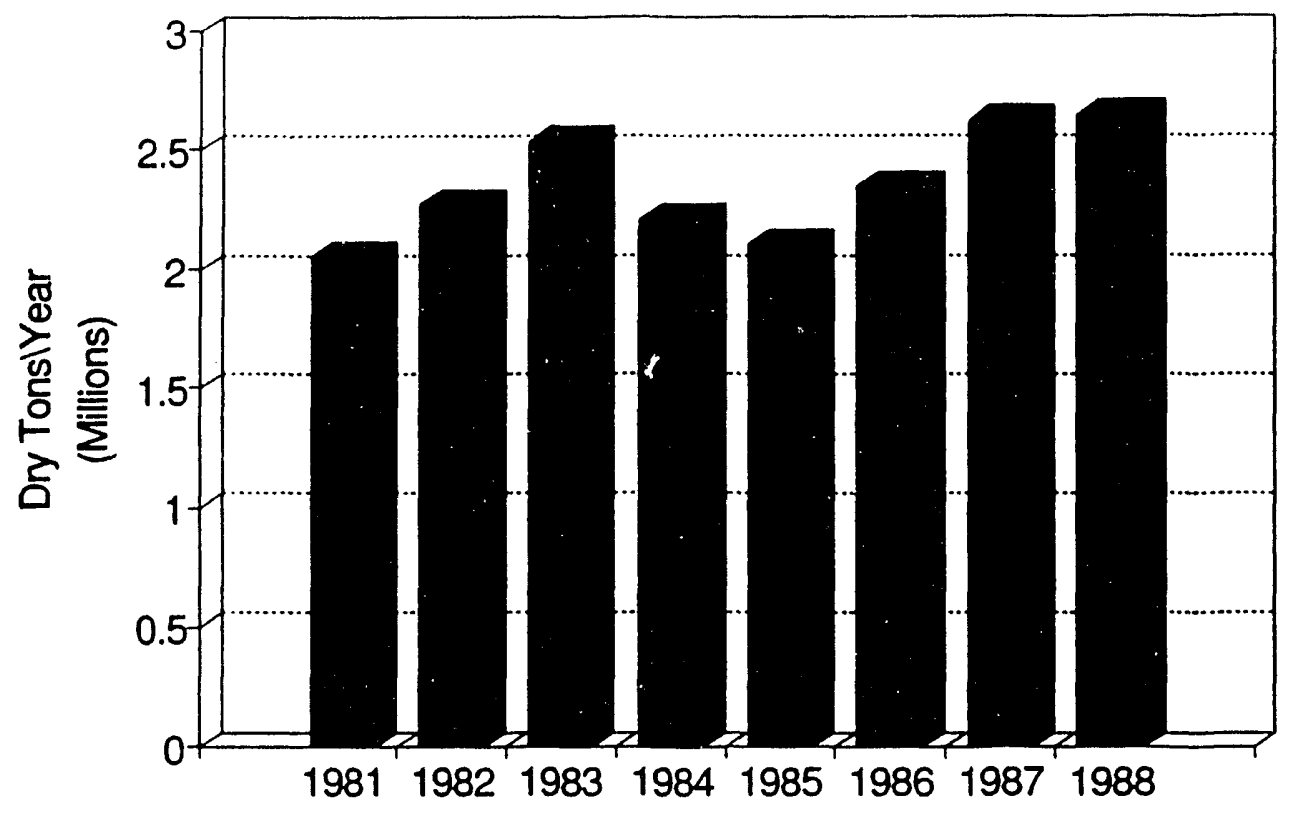

Figure 1-8

Total Annual Logging Residues on

National Forest Lands, 1981-1988

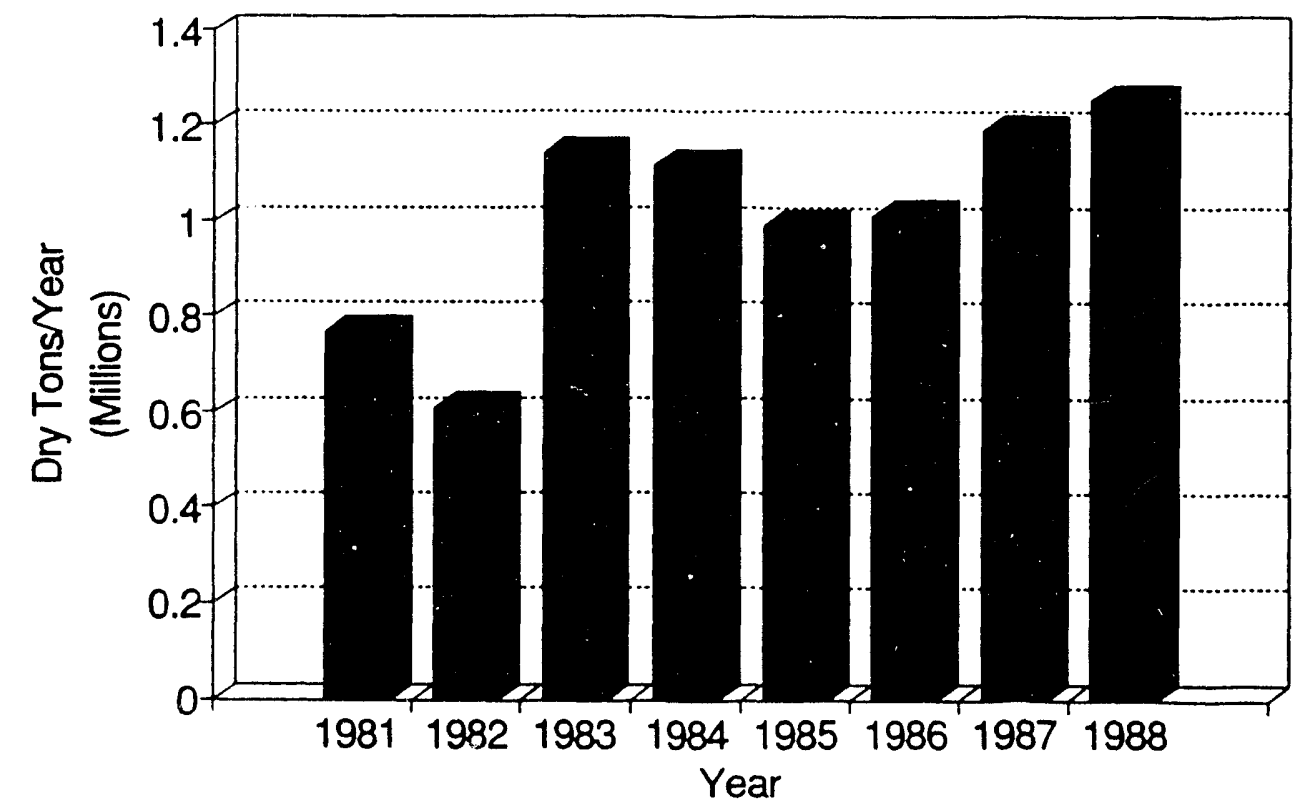


Figure 1-9

Total Annual Logging Residues on State Forest Lands, 1981-1988

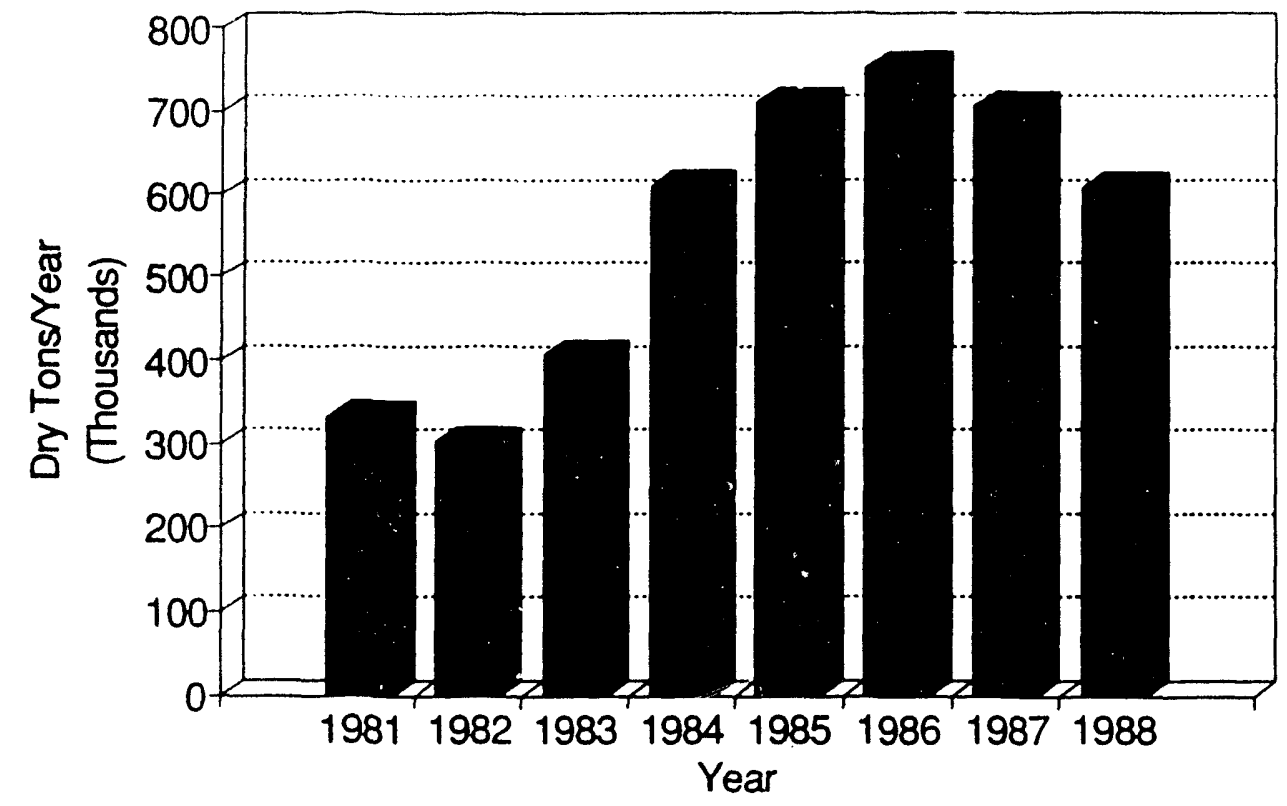

Figure I-10

\section{Total Annual Logging Residues on} Indian Forest Lands, 1981-1988

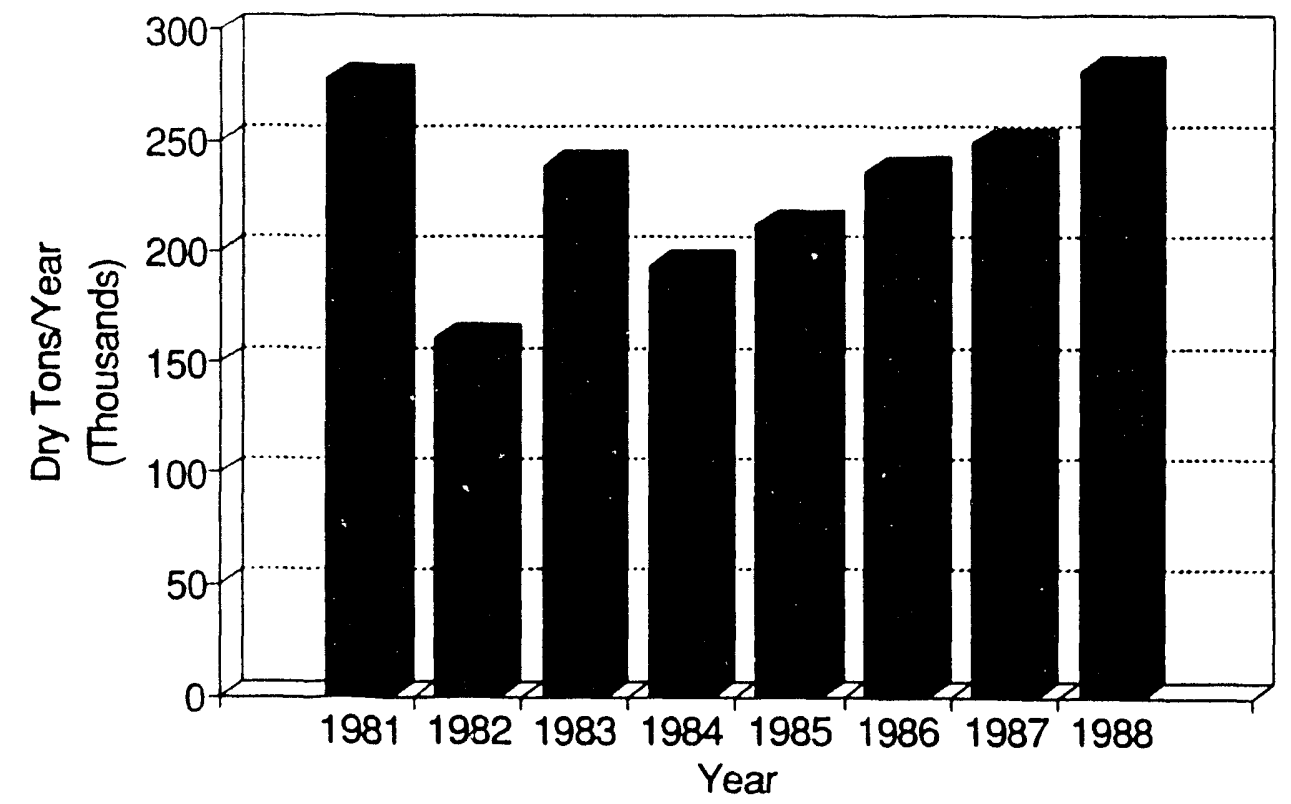


Figure l-11

Total Annual Logging Residues on Other Federal \& Non-Federal Forests, 1981-88

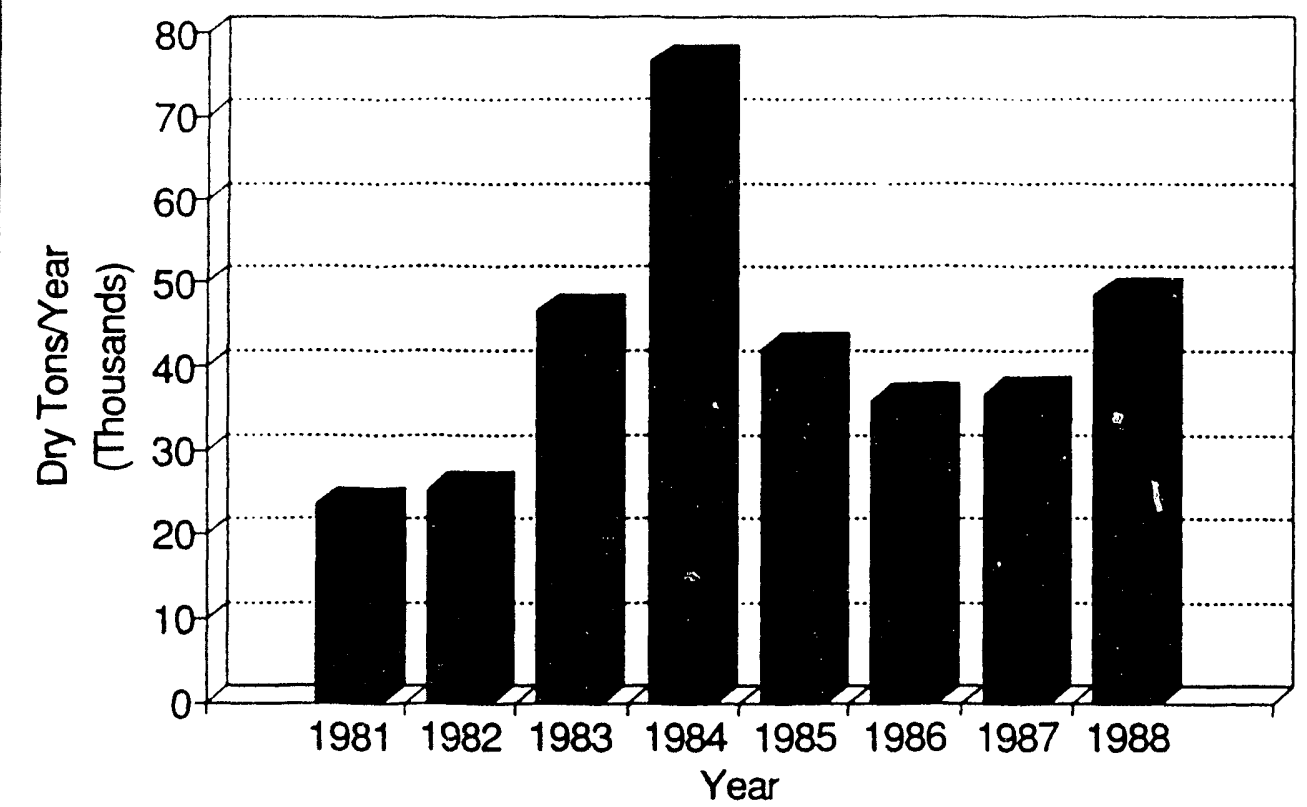




\section{Map |-11}

\section{Total Logging Residues}

Annual Average

Dry Tons (Tnousanas)

8y Reg:on

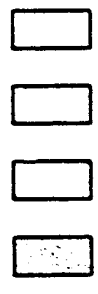

$22 \quad 10 \quad 123$

12410427

$426 \quad 10623$

$824: 0 \quad 1193$

- Regional Boundar

..... County Boundary

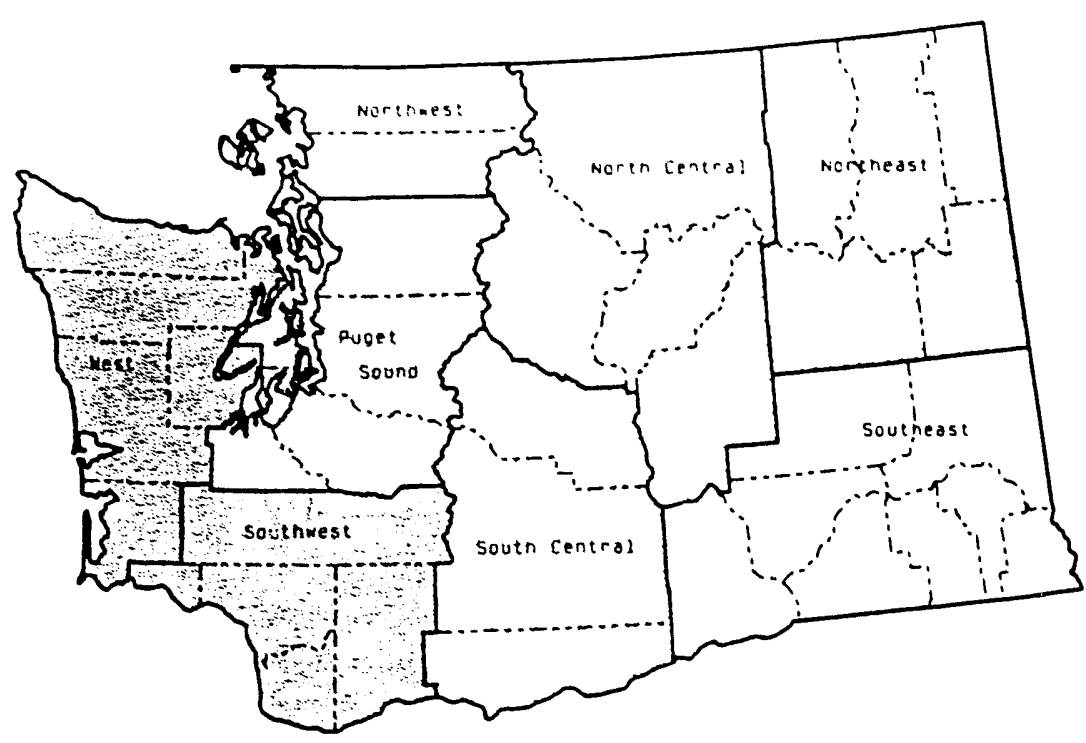

State of Washington

\section{Logging Residues on Private Lands}

Annual Average

Ory Tons (Thousanas)

By Region

$\square \quad 8$ 10 8 !

$\square 82 \quad 10295$

$\square 29610533$

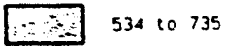

- Regiond! Boulidary County Boundary

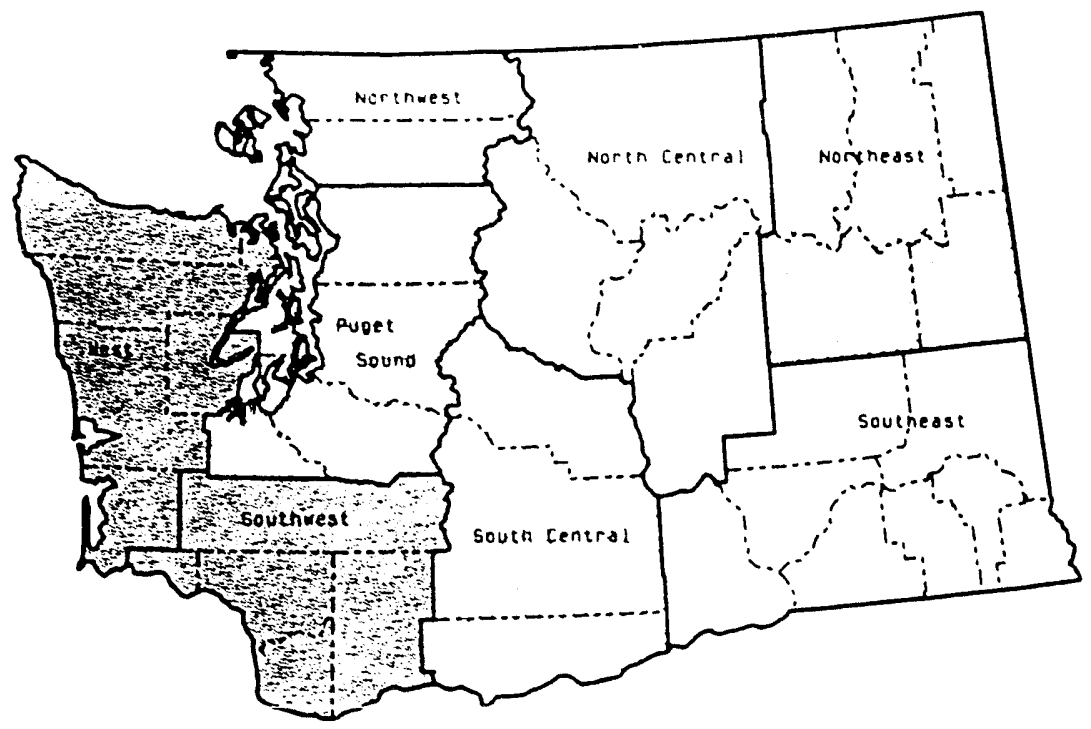

State of Washington 
Map I-13

\section{Logging Residues on National Lands}

Annual Average

Dry Tons (Thousands)

By Region

$\square \quad 131068$

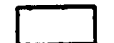

$6910 \quad 122$

$\square 123$ to 230

23110290

Regional Boundary

.... Couney Boundary

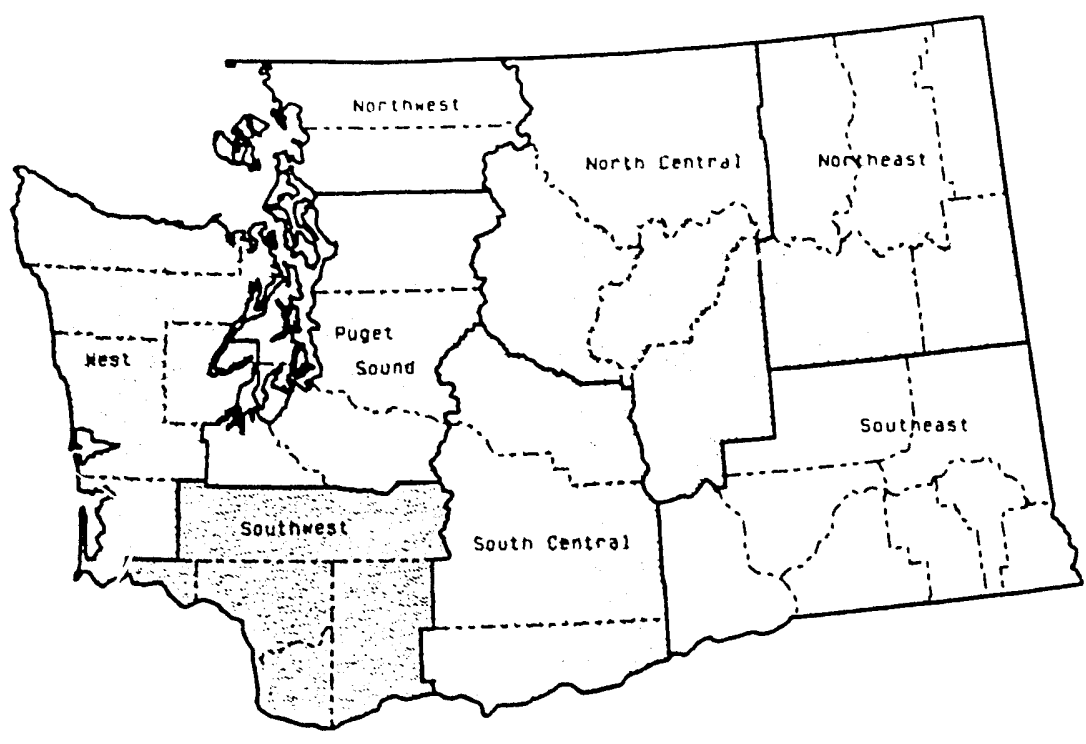

State of Washington

Source masnington State Ded of Natural Resources

Map 1-14

Logging Residues on State Lands

Annual Average

Dry Tons (Tnousands)

By Region

$\square: 1012$

$[131062$

$\square 6310164$

16510238

- Regional Boundary

..... Countr Bounoary

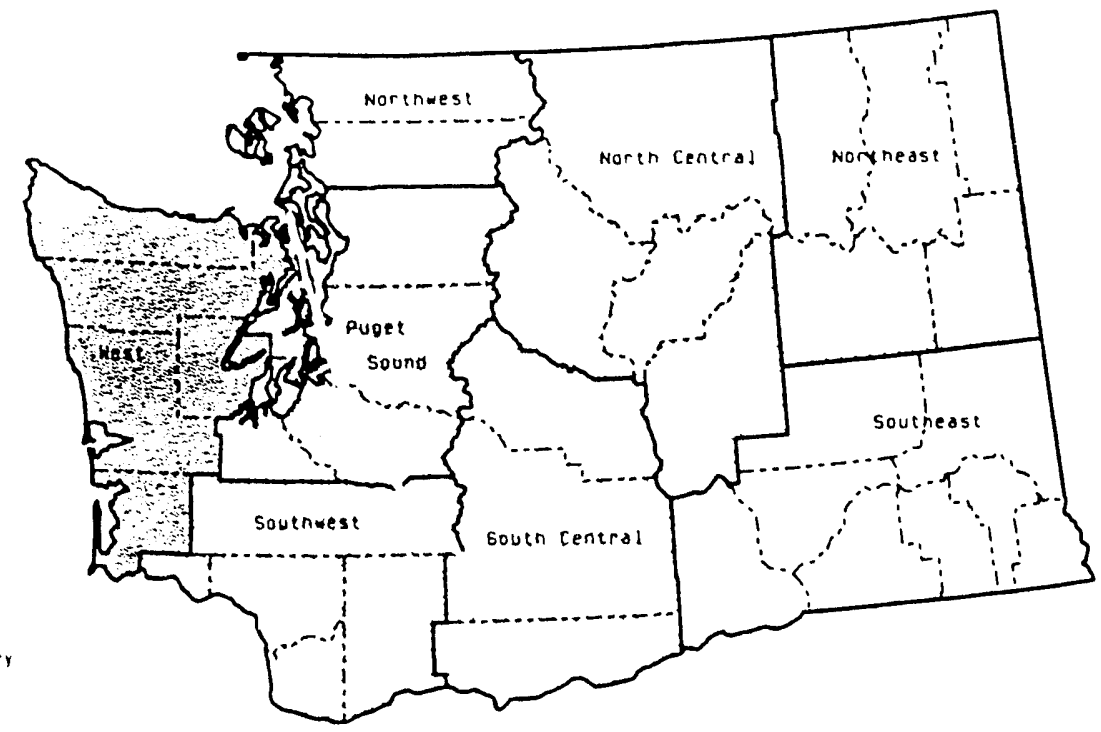

State of Washington

Source wasningion State Ded of Natural Resources 
Map 1-15

\section{Logging Residues on Indian Lands}

Annual Average

Dry Tons (Thousands)

By Region

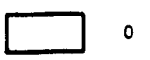

$\square \quad 1$ to 16

$\square 171080$

8110114

- Regional Boundar

....- County Boundary

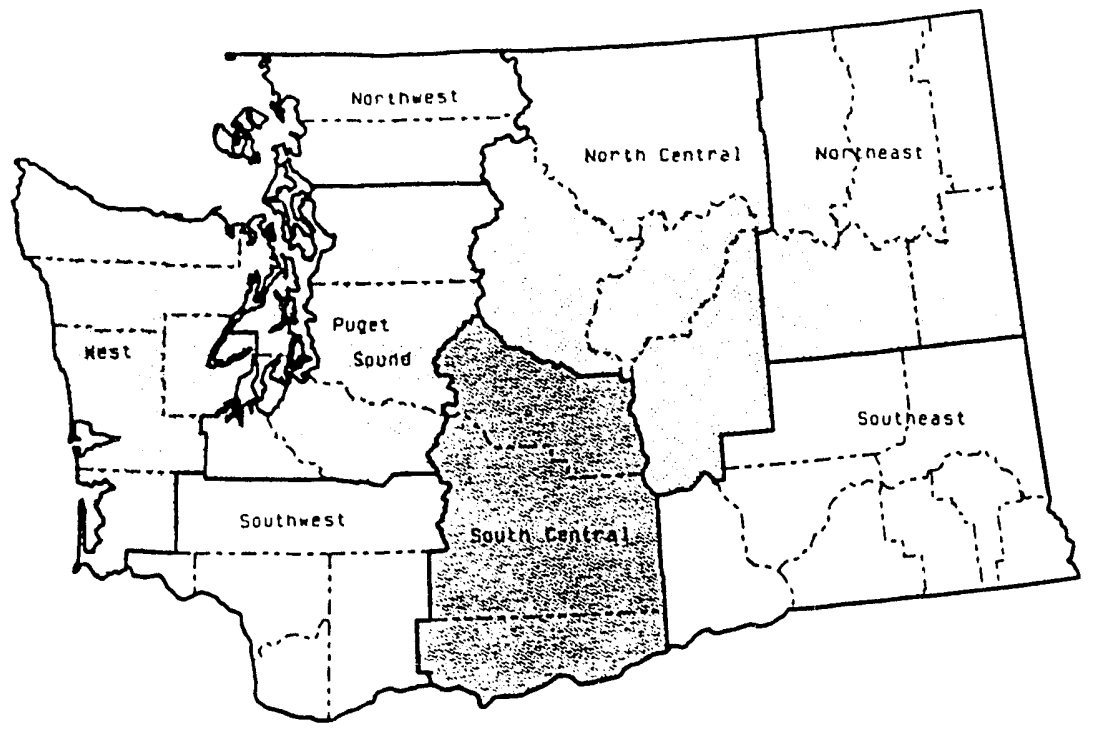

State of Washington

Map 1-16

Logging Residues on Other Federal \& Non-Federal Lands

Annual Average

Dry Tons (Hundreds)

By Region
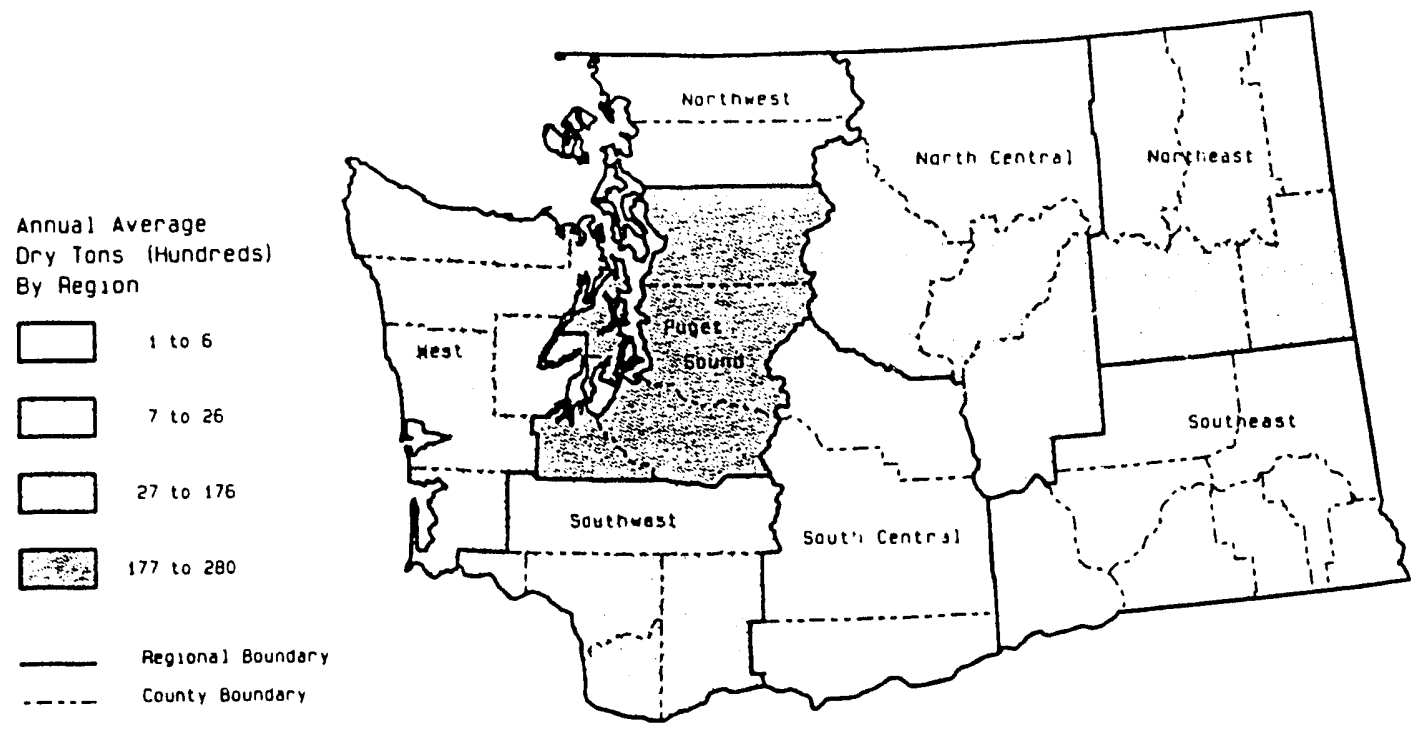

State of Washington

Source washington State Dede. Of Natural Resources 
Table 1-25

\section{Logging Residues on All Forest Land Ownerships by Region}

Ranked by Annual Average (Dry Tons)

\begin{tabular}{|c|c|c|c|c|c|}
\hline \multirow[b]{2}{*}{ Rank } & \multirow[b]{2}{*}{ Region } & \multicolumn{2}{|c|}{-.---Annual Average 1...-. } & \multicolumn{2}{|c|}{-.-.--Range------ } \\
\hline & & (Dry Tons) & $\left(\mathrm{MBtu} /\right.$ Year) ${ }^{2}$ & Minimum & Maximum \\
\hline 1 & West & $1,192,089$ & $21,457,598$ & 907,879 & $1,403,925$ \\
\hline 2 & Southwest & $1,040,758$ & $18,733,643$ & 893,099 & $1,160,065$ \\
\hline 3 & Puget Sound & 606,596 & $10,918,721$ & 443,487 & 767,619 \\
\hline 4 & South Central & 494,378 & $8,898,811$ & 328,164 & 578,855 \\
\hline 5 & Northeast & 362,769 & $6,529,839$ & 241,061 & 473,522 \\
\hline 6 & North Central & 247,242 & $4,450,358$ & 140,302 & 330,243 \\
\hline 7 & Northwest & 223,924 & $4,030,631$ & 164,202 & 263,525 \\
\hline \multirow[t]{2}{*}{8} & Southeast & 22,849 & 411,284 & 12,280 & 32,555 \\
\hline & State & $4,190,605$ & $75,430,886$ & $3,370,809$ & $4,847,3689$ \\
\hline
\end{tabular}

1 Average based on report from 1981-1988

2 Energy Equivalent, $18 \mathrm{MBtu} / \mathrm{ton}$

Source: Washington State Dept. of Natural Resources, and U.S. Dept. of Agriculture

Table I-26

\section{Logging Residues on Private Forest Lands by Region}

Ranked by Annual Average (Dry Tons)

\begin{tabular}{|c|c|c|c|c|c|}
\hline \multirow[b]{2}{*}{ Rank } & \multirow[b]{2}{*}{ Region } & \multicolumn{2}{|c|}{..-.-Annual Average ${ }^{1}$-... } & \multicolumn{2}{|c|}{--.----Range---.-- } \\
\hline & & (Dry Tons) & (MBtu/Year) $^{2}$ & Minimum & Maximum \\
\hline 1 & West & 735.020 & $13,230,355$ & 565,813 & 941,904 \\
\hline 2 & Southwest & 665,134 & $11,972,419$ & 534,578 & 734,003 \\
\hline 3 & Puget Sound & 402,704 & $7,248,678$ & 325,851 & 497,212 \\
\hline 4 & Northeast & 190,054 & $3,420,966$ & 146,473 & 228,743 \\
\hline 5 & South Central & 185,434 & $3,337,815$ & 132,290 & 218,734 \\
\hline 6 & Northwest & 128,828 & $2,318,907$ & 102,361 & 179,935 \\
\hline 7 & North Central & 35,4446 & 37,988 & 21,458 & 49,519 \\
\hline \multirow[t]{2}{*}{8} & Southeast & 8,203 & 147,649 & 3,546 & 12,976 \\
\hline & State & $2,350,821$ & $42,314,776$ & $2,051,948$ & $2,652,232$ \\
\hline
\end{tabular}

1 Average based on report from 1981-1988

2 Energy Equivalent, $18 \mathrm{MBtu} / \mathrm{ton}$

Source: Washington State Dept. of Natural Resources, and U.S. Dept. of Agriculture 
Table 1-27

\section{Logging Residues on National Forest Lands by Region}

Ranked by Annual Average (Dry Tons)

\begin{tabular}{|c|c|c|c|c|c|}
\hline \multirow{2}{*}{\multicolumn{2}{|c|}{ Rank Region }} & \multicolumn{2}{|c|}{-----Annual Average 1 } & \multicolumn{2}{|c|}{--------Range------- } \\
\hline & & (Dry Tons) & (MBtu/Year) $^{2}$ & Minimum & Maximum \\
\hline 1 & Southwest & 282,803 & $5,090,456$ & 194,843 & 404,741 \\
\hline 2 & West & 178,182 & $3,207,270$ & 114,419 & 251,421 \\
\hline 3 & North Central & 157,849 & $2,841,280$ & 73,162 & 247,444 \\
\hline 4 & South Central & 140,749 & $2,533,475$ & 81,611 & 217,411 \\
\hline 5 & Puget Sound & 104,123 & $1,874,212$ & 46,920 & 154,163 \\
\hline 6 & Northeast & 93,062 & $1,675,116$ & 46,596 & 140,691 \\
\hline 7 & Northwest & 42,010 & 756,176 & 25,180 & 62,251 \\
\hline 8 & Southeast & 13,287 & 239,159 & 4,071 & 21,935 \\
\hline & State & $1,012,064$ & $18,217,145$ & 607,262 & $1,012,064$ \\
\hline
\end{tabular}

1 Average based on report from 1981-1988

2 Energy Equivalent, $18 \mathrm{MBtu} / \mathrm{ton}$

Source: Washington State Dept. of Natural Resources, and U.S. Dept. of Agriculture

Table 1-28

\section{Logging Residues on State Forest Lands by Region}

Ranked by Annual Average (Dry Tons)

\begin{tabular}{|c|c|c|c|c|c|}
\hline \multirow[b]{2}{*}{ Rank } & \multirow[b]{2}{*}{ Region } & \multicolumn{2}{|c|}{-.-Annual Average 1 -...- } & \multicolumn{2}{|c|}{-...-Range-.......- } \\
\hline & & (Dry Tons) & (MBtu/Year) $^{2}$ & Minimum & Maximum \\
\hline 1 & West & 237,952 & $4,283,137$ & 150,0223 & 47,646 \\
\hline 2 & Southwest & 91,394 & $1,645,088$ & 59,184 & 129,107 \\
\hline 3 & Puget Sound & 68,368 & $1,230,623$ & 30,737 & 113,506 \\
\hline 4 & South Central & 55,315 & 995,670 & 4,162 & 95,206 \\
\hline 5 & Northwest & 51,721 & 930,982 & 22,815 & 76,759 \\
\hline 6 & Northeast & 26,535 & 477,625 & 9,615 & 36,167 \\
\hline 7 & North Central & 21,927 & 394,686 & 6,087 & 34,811 \\
\hline \multirow[t]{2}{*}{0} & Southeast & 1,063 & 19,132 & 31 & 2,617 \\
\hline & State & 554,275 & $9,976,944$ & 302,071 & 752,587 \\
\hline
\end{tabular}

1 Average based on report from 1981-1988

2 Energy Equivalent, $18 \mathrm{MBtu} / \mathrm{ton}$

Source: Washington State Dept. of Natural Resources, and U.S. Dept. of Agriculture 
Table 1-29

\section{Logging Residues on Indian Forest Lands by Region}

Ranked by Annual Average (Dry Tons)

\begin{tabular}{|c|c|c|c|c|c|}
\hline \multirow[b]{2}{*}{ Rank } & \multirow[b]{2}{*}{ Region } & \multicolumn{2}{|c|}{....-Annual Average 1 -.... } & \multicolumn{2}{|c|}{ 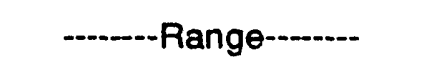 } \\
\hline & & (Dry Tons) & (MBtu/Year) $)^{2}$ & Minimum & Maximum \\
\hline 1 & South Central & 112,690 & $2,028,421$ & 67,929 & 161,645 \\
\hline 2 & Northeast & 49,439 & 889,909 & 27,209 & 73,878 \\
\hline 3 & West & 33,761 & 607,705 & 13,134 & 52,240 \\
\hline 4 & North Central & 31,067 & 559,208 & 24,132 & 40,556 \\
\hline 5 & Puget Sound & 3,421 & 61,577 & 799 & 8,080 \\
\hline 6 & Northwest & 1,061 & 19,106 & 527 & 1,856 \\
\hline 7 & Southeast & 0 & 0 & 0 & 0 \\
\hline \multirow[t]{2}{*}{8} & Southwest & 0 & 0 & 0 & 0 \\
\hline & State & 231,440 & $4,165,927$ & 160,766 & 281,417 \\
\hline
\end{tabular}

1 Average based on report from 1981-1988

2 Energy Equivalent, $18 \mathrm{MBtu} / \mathrm{ton}$

Source: Washington State Dept. of Natural Resources, and U.S. Dept. of Agriculture

Table $1-30$

\section{Logging Residues on Other Federal and Non-Federal Forest Lands by Region}

Ranked by Annual Average (Dry Tons)

\begin{tabular}{|c|c|c|c|c|c|}
\hline \multirow[b]{2}{*}{ Rank } & \multirow[b]{2}{*}{ Region } & \multicolumn{2}{|c|}{...-Annual Average ${ }^{1}$} & \multicolumn{2}{|c|}{-...-..-Range--..... } \\
\hline & & (Dry Tons) & (MBtu/Year) $^{2}$ & Minimum & Maximum \\
\hline 1 & Puget Sound & 27,980 & 503,631 & 7,081 & 93,885 \\
\hline 2 & West & 7,174 & 129,130 & 0 & 22,117 \\
\hline 3 & Northeast & 3,679 & 66,223 & 0 & 16,406 \\
\hline 4 & Southwest & 1,427 & 25,680 & 0 & 4,669 \\
\hline 5 & North Central & 955 & 17,195 & 0 & 5,722 \\
\hline 6 & Northwest & 303 & 5,460 & 0 & 1,052 \\
\hline 7 & Southeast & 297 & 5,345 & 0 & 1,289 \\
\hline \multirow[t]{2}{*}{8} & South Central & 191 & 3,429 & 0 & 699 \\
\hline & State & 42,005 & 756,093 & 23,588 & 76,686 \\
\hline
\end{tabular}

1 Average based on report from 1981-1988

2 Energy Equivalent, $18 \mathrm{MBtu} / \mathrm{ton}$

Source: Washington State Dept. of Natural Resources, and U.S. Dept. of Agriculture 
Table 1-31

\section{Logging Residues - All Forest Land Ownerships by County}

Ranked by Annual Average (Dry Tons)

\begin{tabular}{|c|c|c|c|c|c|}
\hline \multirow[b]{2}{*}{ Rank } & \multirow[b]{2}{*}{ County (Region) } & \multicolumn{2}{|c|}{-.---Annual Average 1..-- } & \multicolumn{2}{|c|}{ 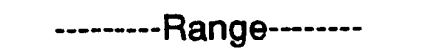 } \\
\hline & & (Dry Tons) $^{2}$ & (MBtu/Year) & Minimum & Maximum \\
\hline 1 & Lewis (SW) & 407,760 & $7,339,686$ & 285,380 & 532,532 \\
\hline 2 & Grays Harbor (W) & 402,872 & $7,251,695$ & 212,317 & 522,958 \\
\hline 3 & Cowliz (SW) & 287,960 & $5,183,283$ & 208.621 & 376,995 \\
\hline 4 & Jefferson $(W)$ & 280,852 & $5,055,341$ & 207,000 & 347,464 \\
\hline 5 & Skamania (SW) & 253,720 & $4,566,955$ & 208,024 & 336,950 \\
\hline 6 & Clallam $(W)$ & 231,455 & $4,166,183$ & 165,040 & 303,419 \\
\hline 7 & Yakima (SC) & 213,075 & $3,835,358$ & 139,796 & 253,263 \\
\hline 8 & Kittitas (SC) & 190,044 & $3,420,797$ & 118,228 & 244,799 \\
\hline 9 & Pierce (PS) & 181,289 & $3,263,209$ & 147,709 & 237,028 \\
\hline 10 & King (PS) & 171,010 & $3,078,176$ & 133,201 & 211,444 \\
\hline 11 & Okanogan (NC) & 157,695 & $2,838,512$ & 77,268 & 229,960 \\
\hline 12 & Mason (W) & 157,284 & $2,831,120$ & 79,643 & 243,503 \\
\hline 13 & Skagit (NW) & 144,625 & $2,603,252$ & 116,882 & 175,200 \\
\hline 14 & Snohomish (PS) & 135,968 & $2,447,419$ & 70,754 & 190,365 \\
\hline 15 & Stevens (NE) & 131,620 & $2,369,153$ & 94,668 & 162,461 \\
\hline 16 & Pacific (W) & 119,625 & $2,153,259$ & 64,939 & 163,213 \\
\hline 17 & Ferry (NE) & 115,745 & $2,083,417$ & 80,059 & 154,634 \\
\hline 18 & Klickitat (SC) & 91,259 & $1,642,656$ & 64,322 & 126,469 \\
\hline 19 & Chelan (NC) & 89,393 & $1,609,073$ & 62,735 & 117,305 \\
\hline 20 & Pend Oreille (NE) & 86,711 & $1,560,791$ & 49,233 & 129,691 \\
\hline 21 & Thurston (PS) & 75,124 & $1,352,229$ & 51,302 & 122,651 \\
\hline 22 & Whatcom (NW) & 59,704 & $1,074,673$ & 31,430 & 77,995 \\
\hline 23 & Wahkiakum (SW) & 46,921 & 844,577 & 34,811 & 57,480 \\
\hline 24 & Clark (SW) & 44,397 & 799,143 & 9,880 & 93,724 \\
\hline 25 & Kitsap (PS) & 43,205 & 777,687 & 31,330 & 57,135 \\
\hline 26 & Spokane (NE) & 25,398 & 457,162 & 13,367 & 30,732 \\
\hline 27 & Island (NW) & 15,298 & 275,369 & 7,510 & 30,296 \\
\hline 28 & Columbia (SE) & 11,342 & 204,149 & 3,475 & 21,400 \\
\hline 29 & Asotin (SE) & 7,451 & 134,109 & 1,622 & 17,645 \\
\hline 30 & San Juan (NW) & 4,297 & 77,337 & 1,955 & 8,728 \\
\hline 31 & Lincoln (NE) & 3,295 & 59,316 & 1,594 & 4,217 \\
\hline 32 & Garfield (SE) & 1,974 & 35,524 & 86 & 5,013 \\
\hline 33 & Walla Walla (SE) & 1,423 & 25,608 & 67 & 3,756 \\
\hline 34 & Whitman (SE) & 602 & 10,844 & 125 & 1,147 \\
\hline 35 & Douglas (NC) & 154 & 2,774 & 0 & 935 \\
\hline 36 & Benton (SE) & 58 & 1,050 & 0 & 467 \\
\hline NA & Adams (SE) & 0 & 0 & 0 & 0 \\
\hline NA & Franklin (SE) & 0 & 0 & 0 & 0 \\
\hline \multirow[t]{2}{*}{ NA } & Grant (NC) & 0 & 0 & 0 & 0 \\
\hline & State & $4,190,605$ & $75,430,886$ & $3,370,809$ & $4,847,689$ \\
\hline
\end{tabular}

1 Average based on reports from 1981-1988

2 Energy Equivalent, $18 \mathrm{MBtu} / \mathrm{ton}$

Source: Washington State Dept. of Natural Resources, and U.S. Dept. of Agriculture 
Table $1-32$

\section{Logging Residues on Private Forest Lands by County}

Ranked by Annual Average (Dry Tons)

\begin{tabular}{|c|c|c|c|c|c|}
\hline \multirow[b]{2}{*}{ Rank } & \multirow[b]{2}{*}{ County (Region) } & \multicolumn{2}{|c|}{--.-Annual Average 1 -.... } & \multicolumn{2}{|c|}{ 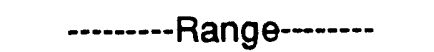 } \\
\hline & & (Dry Tons) & $(\text { MBtu/Year })^{2}$ & Minimum & Maximum \\
\hline 1 & Grays Harbor (W) & 313,335 & $5,640,038$ & 158,759 & 442,677 \\
\hline 2 & Lewis (SW) & 278,970 & $5,021,458$ & 205,424 & 304,508 \\
\hline 3 & Cowlitz (SW) & 246,549 & $4,437,874$ & 182,436 & 321,739 \\
\hline 4 & Pierce (PS) & 123,654 & $2,225,765$ & 96,442 & 186,771 \\
\hline 5 & King (PS) & 118,510 & $2,133,171$ & 94,618 & 160,871 \\
\hline 6 & Clallam $(W)$ & 114,497 & $2,060,947$ & 98,151 & 128,031 \\
\hline 7 & Kittitas (SC) & 107,363 & $1,932,532$ & 68,668 & 142,817 \\
\hline 8 & Mason (W) & 107,090 & $1,927,623$ & 29,456 & 189,242 \\
\hline 9 & Pacific (W) & 105,190 & $1,893,419$ & 54,796 & 153,073 \\
\hline 10 & Jefferson $(W)$ & 94,907 & $1,708,327$ & 53,610 & 131,566 \\
\hline 11 & Stevens (NE) & 84,017 & $1,512,308$ & 71,054 & 99,596 \\
\hline 12 & Skagit (NW) & 81,948 & $1,475,071$ & 67,569 & 106,186 \\
\hline 13 & Snohomish (PS) & 69,765 & $1,255,773$ & 45,840 & 109,961 \\
\hline 14 & Skamania (SW) & 58,510 & $1,053,179$ & 24,930 & 91,541 \\
\hline 15 & Klickitat (SC) & 55,168 & 993,028 & 43,379 & 66,990 \\
\hline 16 & Thurston (PS) & 53,538 & 963,688 & 33,380 & 95,393 \\
\hline 17 & Pend Oreille (NE) & 47,284 & 851,114 & 32,963 & 71,343 \\
\hline 18 & Wahkiakum (SW) & 42,199 & 759,576 & 31,201 & 57,013 \\
\hline 19 & Clark (SW) & 38,907 & 700,332 & 8,814 & 81,587 \\
\hline 20 & Kitsap (PS) & 37,238 & 670,281 & 28,004 & 50,261 \\
\hline 21 & Ferry (NE) & 32,650 & 587,706 & 22,687 & 42,851 \\
\hline 22 & Whatcom (NW) & 28,909 & 520,360 & 17,380 & 40,222 \\
\hline 23 & Spokane (NE) & 23,175 & 417,149 & 12,302 & 28,821 \\
\hline 24 & Yakima (SC) & 22,903 & 412,254 & 15,460 & 38,699 \\
\hline 25 & Okanogan (NC) & 20,616 & 371,096 & 10,433 & 31,159 \\
\hline 26 & Chelan (NC) & 14,673 & 264,118 & 3,548 & 23,920 \\
\hline 27 & Island (NW) & 13,740 & 247,316 & 6,711 & 27,651 \\
\hline 28 & San Juan (NW) & 4,231 & 76.161 & 1,955 & 8,537 \\
\hline 29 & Columbia (SE) & 4,131 & 74,354 & 2,218 & 8,381 \\
\hline 30 & Lincoln (NE) & 2,927 & 52,688 & 1,594 & 4,217 \\
\hline 31 & Asotin (SE) & 1,202 & 21,643 & 651 & 3,239 \\
\hline 32 & Garfield (SE) & 1,161 & 20,900 & 0 & 2,702 \\
\hline 33 & Walla Walla (SE) & 1,048 & 18,859 & 19 & 3,756 \\
\hline 34 & Whitman (SE) & 602 & 10,844 & 125 & 1,147 \\
\hline 35 & Douglas (NC) & 154 & 2,774 & 0 & 935 \\
\hline 36 & Benton (SE) & 58 & 1,050 & 0 & 467 \\
\hline NA & Adams (SE) & 0 & 0 & 0 & 0 \\
\hline NA & Franklin (SE) & 0 & 0 & 0 & 0 \\
\hline \multirow[t]{2}{*}{ NA } & Grant (NC) & 0 & 0 & 0 & 0 \\
\hline & State & $2,350,821$ & $42,314,776$ & $2,051,948$ & $2,652,232$ \\
\hline
\end{tabular}

1 Average based on reports from 1981-1988

2 Energy Equivalent, $18 \mathrm{MBtu} / \mathrm{ton}$

Source: Washington State Dept. of Natural Resources, and U.S. Dept. of Agriculture 
Table 1-33

Logging Residues on National Forest Lands By County

Ranked by Annual Average (Dry Tons)

\begin{tabular}{|c|c|c|c|c|c|}
\hline \multirow[b]{2}{*}{ Rank } & \multirow[b]{2}{*}{ County (Region) } & \multicolumn{2}{|c|}{----Annual Average 1...- } & \multicolumn{2}{|c|}{ 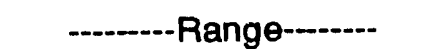 } \\
\hline & & (Dry Tons) & $($ MBtu/Year) 2 & Minimum & Maximum \\
\hline 1 & Skamania (SW) & 182,570 & $3,286,254$ & 133,553 & 274,396 \\
\hline 2 & Lewis (SW) & 97,078 & $1,747,405$ & 22,423 & 204,244 \\
\hline 3 & Okanogan (NC) & 85,562 & $1,540,109$ & 36,729 & 151,609 \\
\hline 4 & Kittitas (SC) & 74,213 & $1,335,826$ & 28,944 & 117,761 \\
\hline 5 & Chelan (NC) & 72,287 & $1,301,171$ & 36,433 & 104,827 \\
\hline 6 & Yakima (SC) & 62,512 & $1,125,211$ & 39,041 & 98,792 \\
\hline 7 & Clallam (W) & 61,211 & $1,101,800$ & 35,993 & 119,804 \\
\hline 8 & Jefferson $(W)$ & 42,127 & 758,283 & 23,264 & 75,420 \\
\hline 9 & King (PS) & 40,456 & 728,201 & 17,971 & 68,296 \\
\hline 10 & Mason (W) & 38,838 & 699,093 & 15,910 & 61,662 \\
\hline 11 & Grays Harbor (W) & 36,005 & 648,095 & 13,468 & 58,552 \\
\hline 12 & Ferry (NE) & 35,110 & 631,984 & 13,231 & 50,720 \\
\hline 13 & Pend Oreille (NE) & 34,353 & 618,358 & 15,297 & 57,995 \\
\hline 14 & Snohomish (PS) & 32,572 & 586,305 & 0 & 51,847 \\
\hline 15 & Plerce (PS) & 31,095 & 559,706 & 9,705 & 62,099 \\
\hline 16 & Skagit (NW) & 25,987 & 467,762 & 16,231 & 31,603 \\
\hline 17 & Stevens (NE) & 23,556 & 424,005 & 9,942 & 42,111 \\
\hline 18 & Whatcom (NW) & 16,023 & 288,414 & 3,322 & 30,794 \\
\hline 19 & Columbia (SE) & 6,484 & 116,713 & 1,257 & 15.617 \\
\hline 20 & Asotin (SE) & 5,990 & 107,822 & 485 & 16,394 \\
\hline 21 & Klickitat (SC) & 4,024 & 72,439 & 0 & 12,483 \\
\hline 22 & Cowlitz (SW) & 3,155 & 56,796 & 578 & 6,741 \\
\hline 23 & Garfield (SE) & 812 & 14,624 & 0 & 3,601 \\
\hline 24 & Spokane (NE) & 43 & 770 & 0 & 342 \\
\hline NA & Adams (SE) & 0 & 0 & 0 & 0 \\
\hline NA & Benton (SE) & 0 & 0 & 0 & 0 \\
\hline NA & Clark (SW) & 0 & 0 & 0 & 0 \\
\hline NA & Douglas (NC) & 0 & 0 & 0 & 0 \\
\hline NA & Franklin (SE) & 0 & 0 & 0 & 0 \\
\hline NA & Grant (NC) & 0 & 0 & 0 & 0 \\
\hline NA & Island (NW) & 0 & 0 & 0 & 0 \\
\hline NA & Kitsap (PS) & 0 & 0 & 0 & 0 \\
\hline NA & Lincoln (NE) & 0 & 0 & 0 & 0 \\
\hline NA & Pacific (W) & 0 & 0 & 0 & 0 \\
\hline NA & San Juan (NW) & 0 & 0 & 0 & 0 \\
\hline NA & Thurston (PS) & 0 & 0 & 0 & 0 \\
\hline NA & Wahkiakum (SW) & 0 & 0 & 0 & 0 \\
\hline NA & Walla Walla (SE) & 0 & 0 & 0 & 0 \\
\hline \multirow[t]{2}{*}{ NA } & Whitman (SE) & 0 & 0 & 0 & 0 \\
\hline & State & $1,012,064$ & $18,217,145$ & 607,262 & $1,012,064$ \\
\hline
\end{tabular}

1 Average based on reports from 1981-1988

2 Energy Equivalent, $18 \mathrm{MBtu} / \mathrm{ton}$

Source: Washington State Dept. of Natural Resources, and U.S. Dept. of $f$.ariculture 
Table 1-34

\section{Logging Residues on State Forest Lands by County}

Ranked by Annual Average (Dry Tons)

\begin{tabular}{|c|c|c|c|c|c|}
\hline \multirow[b]{2}{*}{ Rank } & \multirow[b]{2}{*}{ County (Region) } & \multicolumn{2}{|c|}{-..--Annual Average 1 -.... } & \multicolumn{2}{|c|}{ 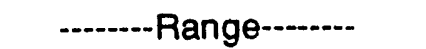 } \\
\hline & & (Dry Tons) & $\left(\right.$ MBtu/Year) ${ }^{2}$ & Minimum & Maximum \\
\hline 1 & Jefferson $(W)$ & 143,035 & $2,574,627$ & 105,896 & 203,938 \\
\hline 2 & Clallam $(W)$ & 49,526 & 891,468 & 15,577 & 94,146 \\
\hline 3 & Cowlitz (SW) & 37,857 & 681,428 & 18,122 & 52,434 \\
\hline 4 & Skagit (NW) & 36,293 & 653,280 & 12,548 & 54,366 \\
\hline 5 & Lewis (SW) & 31,594 & 568,684 & 9,178 & 52,851 \\
\hline 6 & Snohomish (PS) & 31,348 & 564,267 & 8,735 & 56,790 \\
\hline 7 & Klickitat (SC) & 24,291 & 437,244 & 1,121 & 39,715 \\
\hline 8 & Yakima (SC) & 22,555 & 405.993 & 1,275 & 39,349 \\
\hline 9 & Grays Harbor (W) & 20,444 & 367,995 & 2,262 & 49,622 \\
\hline 10 & Okanogan (NC) & 19,842 & 357,162 & 5,396 & 34,811 \\
\hline 11 & Stevens (NE) & 19,259 & 346,662 & 7,163 & 26,376 \\
\hline 12 & Thurston (PS) & 15,420 & 277,552 & 2,890 & 31,302 \\
\hline 13 & Whatcom (NW) & 14,082 & 253,471 & 6,652 & 22,250 \\
\hline 14 & Pacific (W) & 14,012 & 252,216 & 5,099 & 29,900 \\
\hline 15 & Skamania (SW) & 12,251 & 220,517 & 762 & 25,442 \\
\hline 16 & Mason (W) & 10,935 & 196,832 & 2,706 & 20,407 \\
\hline 17 & King (PS) & 9,837 & 177,057 & 1,053 & 19,374 \\
\hline 18 & Pierce (PS) & 8,982 & 161,676 & 2,566 & 21,137 \\
\hline 19 & Kittitas (SC) & 8,468 & 152,433 & 1,766 & 17,952 \\
\hline 20 & Clark (SW) & 5,066 & 91,185 & 1,057 & 12,090 \\
\hline 21 & Wahkiakum (SW) & 4,626 & 83,274 & 467 & 12,646 \\
\hline 22 & Pend Oreille (NE) & 4,292 & 77,252 & 878 & 8,976 \\
\hline 23 & Kitsap (PS) & 2,782 & 50,070 & 1,642 & 4,659 \\
\hline 24 & Chelan (NC) & 2,085 & 37,524 & 0 & 5,661 \\
\hline 25 & Spokane (NE) & 1,434 & 25,809 & 131 & 2,870 \\
\hline 26 & Island (NW) & 1,315 & 23,678 & 0 & 4,476 \\
\hline 27 & Ferry (NE) & 1,182 & 21,275 & 0 & 2,569 \\
\hline 28 & Columbia (SE) & 430 & 7,737 & 0 & 1,566 \\
\hline 29 & Walla Walla (SE) & 375 & 6,750 & 0 & 2,617 \\
\hline 30 & Lincoln (NE) & 368 & 6,628 & 0 & 1,074 \\
\hline 31 & Asotin (SE) & 258 & 4,645 & 0 & 1,465 \\
\hline 32 & San Juan (NW) & 31 & 552 & 0 & 245 \\
\hline NA & Adams (SE) & 0 & 0 & 0 & 0 \\
\hline NA & Benton (SE) & 0 & 0 & 0 & 0 \\
\hline NA & Douglas (NC) & 0 & 0 & 0 & 0 \\
\hline NA & Franklin (SE) & 0 & 0 & 0 & 0 \\
\hline NA & Garfield (SE) & 0 & 0 & 0 & 0 \\
\hline NA & Grant (NC) & 0 & 0 & 0 & 0 \\
\hline \multirow[t]{2}{*}{ NA } & Whitman (SE) & 0 & 0 & 0 & 0 \\
\hline & State & 554,275 & $9,976,944$ & 302.071 & 752,587 \\
\hline
\end{tabular}

1 Average based on reports from 1981-1988

2 Energy Equivalent, $18 \mathrm{MBtu} / \mathrm{ton}$

Source: Washington State Dept. of Natural Resources, and U.S. Dept. of Agriculture 
Table 1-35

\section{Logging Residues on Indian Forest Lands by County}

Ranked by Annual Average (Dry Tons)

\begin{tabular}{|c|c|c|c|c|c|}
\hline \multirow[b]{2}{*}{ Rank } & \multirow[b]{2}{*}{ County (Region) } & \multicolumn{2}{|c|}{---Annual Average 1 -.... } & \multicolumn{2}{|c|}{........-Range-.......- } \\
\hline & & (Dry Tons) & $\left(\right.$ MBtu/Year) ${ }^{2}$ & Minimum & Maximum \\
\hline 1 & Yakima (SC) & 105,106 & $1,891,901$ & 66,829 & 138,321 \\
\hline 2 & Ferry (NE) & 46,009 & 828,167 & 27,209 & 65,041 \\
\hline 3 & Okanogan (NC) & 31,067 & 559,208 & 24,132 & 40,556 \\
\hline 4 & Grays Harbor (W) & 27,286 & 491,143 & 12,180 & 42,046 \\
\hline 5 & Klickitat (SC) & 7,584 & 136,521 & 1,100 & 23,325 \\
\hline 6 & Clallam (W) & 6,087 & 109,567 & 954 & 9,210 \\
\hline 7 & Stevens (NE) & 3,341 & 60,137 & 0 & 8,124 \\
\hline 8 & Snohomish (PS) & 2,183 & 39,295 & 636 & 3,175 \\
\hline 9 & Kitsap (PS) & 815 & 14,664 & 164 & 2,421 \\
\hline 10 & Whatcom (NW) & 676 & 12,177 & 333 & 969 \\
\hline 11 & Mason (W) & 389 & 6,995 & 0 & 984 \\
\hline 12 & Skagit (NW) & 385 & 6,930 & 194 & 887 \\
\hline 13 & Thurston (PS) & 367 & 6,612 & 0 & 2,050 \\
\hline 14 & Pend Oreille (NE) & 89 & 1,604 & 0 & 713 \\
\hline 15 & King (PS) & 56 & 1,005 & 0 & 434 \\
\hline NA & Adams (SE) & 0 & 0 & 0 & 0 \\
\hline NA & Asotin (SE) & 0 & 0 & 0 & 0 \\
\hline NA & Benton (SE) & 0 & 0 & 0 & 0 \\
\hline NA & Chelan (NC) & 0 & 0 & 0 & 0 \\
\hline NA & Clark (SW) & 0 & 0 & 0 & 0 \\
\hline NA & Columbia (SE) & 0 & 0 & 0 & 0 \\
\hline NA & Cowiltz (SW) & 0 & 0 & 0 & 0 \\
\hline NA & Douglas (NC) & 0 & 0 & 0 & 0 \\
\hline NA & Franklin (SE) & 0 & 0 & 0 & 0 \\
\hline NA & Garfield (SE) & 0 & 0 & 0 & 0 \\
\hline NA & Grant (NC) & 0 & 0 & 0 & 0 \\
\hline NA & Island (NW) & 0 & 0 & 0 & 0 \\
\hline NA & Jefferson $(W)$ & 0 & 0 & 0 & 0 \\
\hline NA & Kittitas (SC) & 0 & 0 & 0 & 0 \\
\hline NA & Lewis (SW) & 0 & 0 & 0 & 0 \\
\hline NA & Lincoln (NE) & 0 & 0 & 0 & 0 \\
\hline NA & Pacific (W) & 0 & 0 & 0 & 0 \\
\hline NA & Pierce (PS) & 0 & 0 & 0 & 0 \\
\hline NA & San Juan (NW) & 0 & 0 & 0 & 0 \\
\hline NA & Skamania (SW) & 0 & 0 & 0 & 0 \\
\hline NA & Spokane (NE) & 0 & 0 & 0 & 0 \\
\hline NA & Wahkiakum (SW) & 0 & 0 & 0 & 0 \\
\hline NA & Walla Walla (SE) & 0 & 0 & 0 & 0 \\
\hline NA & Whitman (SE) & 0 & 0 & 0 & 0 \\
\hline & State & 231,440 & $4,165,927$ & 160,760 & 281,417 \\
\hline
\end{tabular}

1 Average based on reports from 1981-1988

2 Energy Equivalent, $18 \mathrm{MBtu} / \mathrm{ton}$

Source: Washington State Dept. of Natural Resources, and U.S. Dept. of Agriculture 
Table 1-37

Logging Residues Generated and Amount Available at a Marginal Cost of Less Than $\$ 3.30 / \mathrm{MBtu}$

\begin{tabular}{|c|c|c|c|c|}
\hline Type & Current & 1991-1995 & $\begin{array}{l}\text { Period } \\
1996-2000\end{array}$ & $2001-2010$ \\
\hline Generated & 75.4 & 61.7 & 55.1 & 50.7 \\
\hline Available & 19.2 & 15.3 & 13.6 & 12.75 \\
\hline
\end{tabular}

Reference: Washington State Energy Office 


\section{Mill Residues}

Figures, Maps, and Tables 
Figure I-12

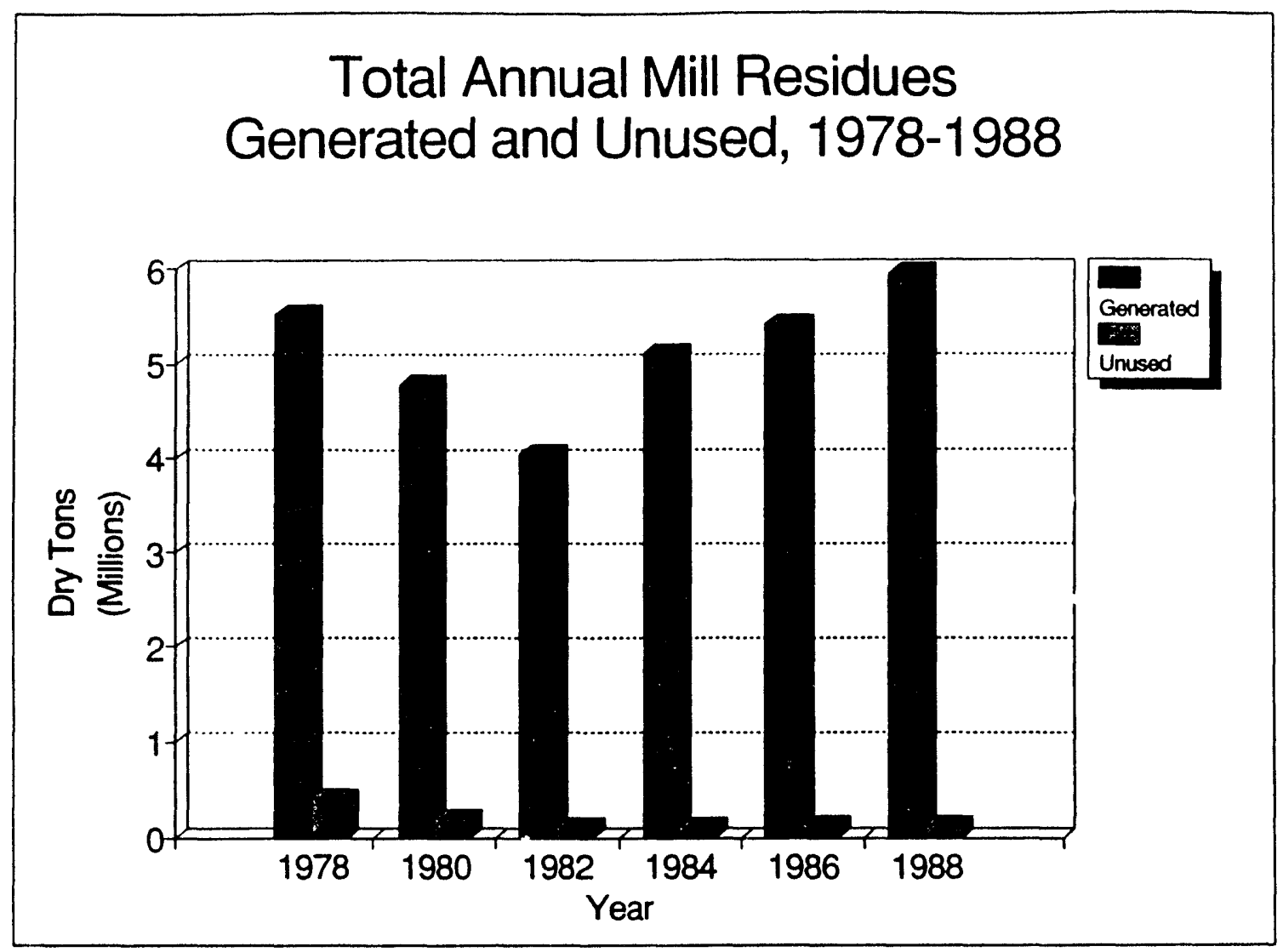

Figure I-13

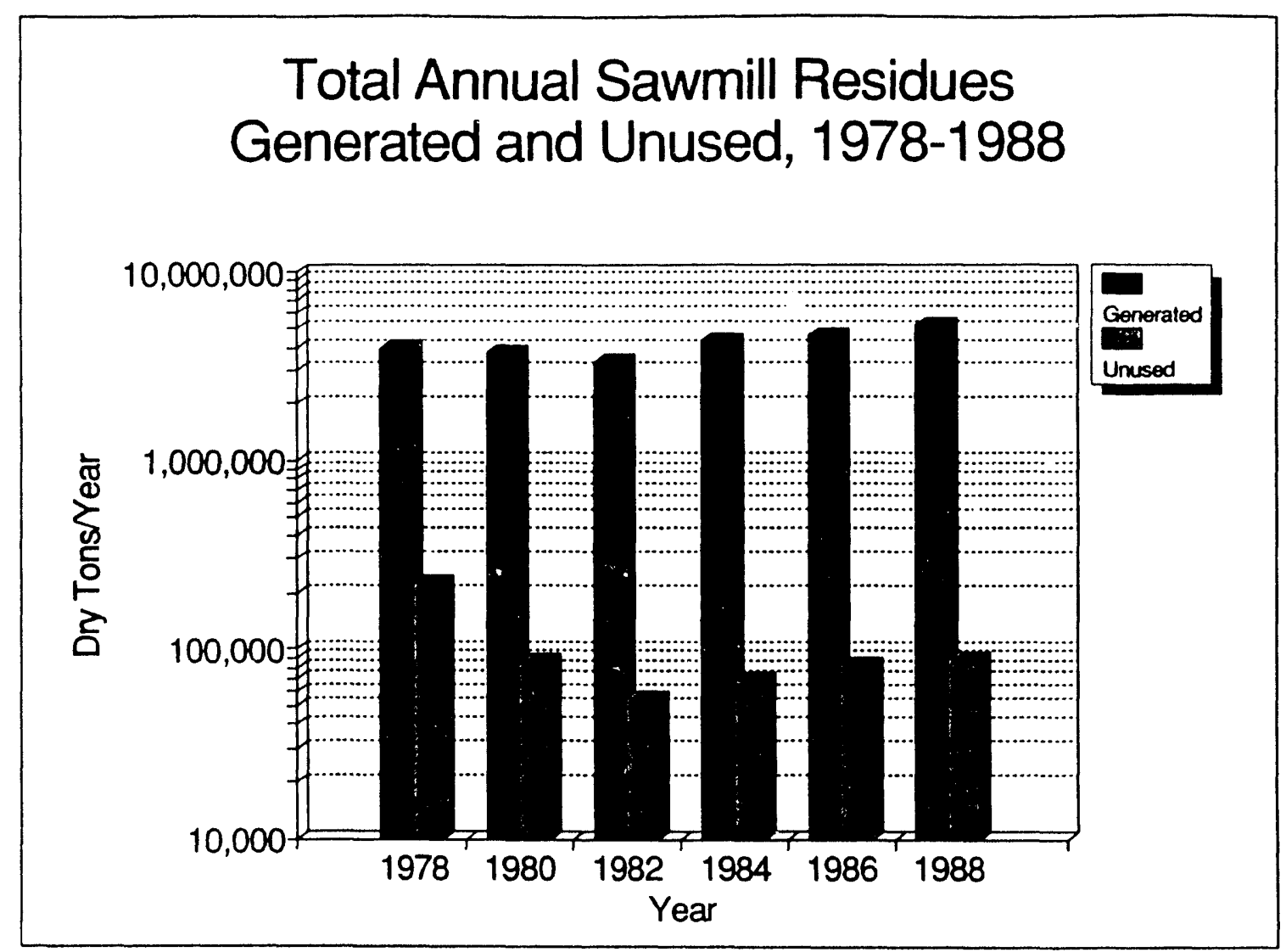


Figure I-14

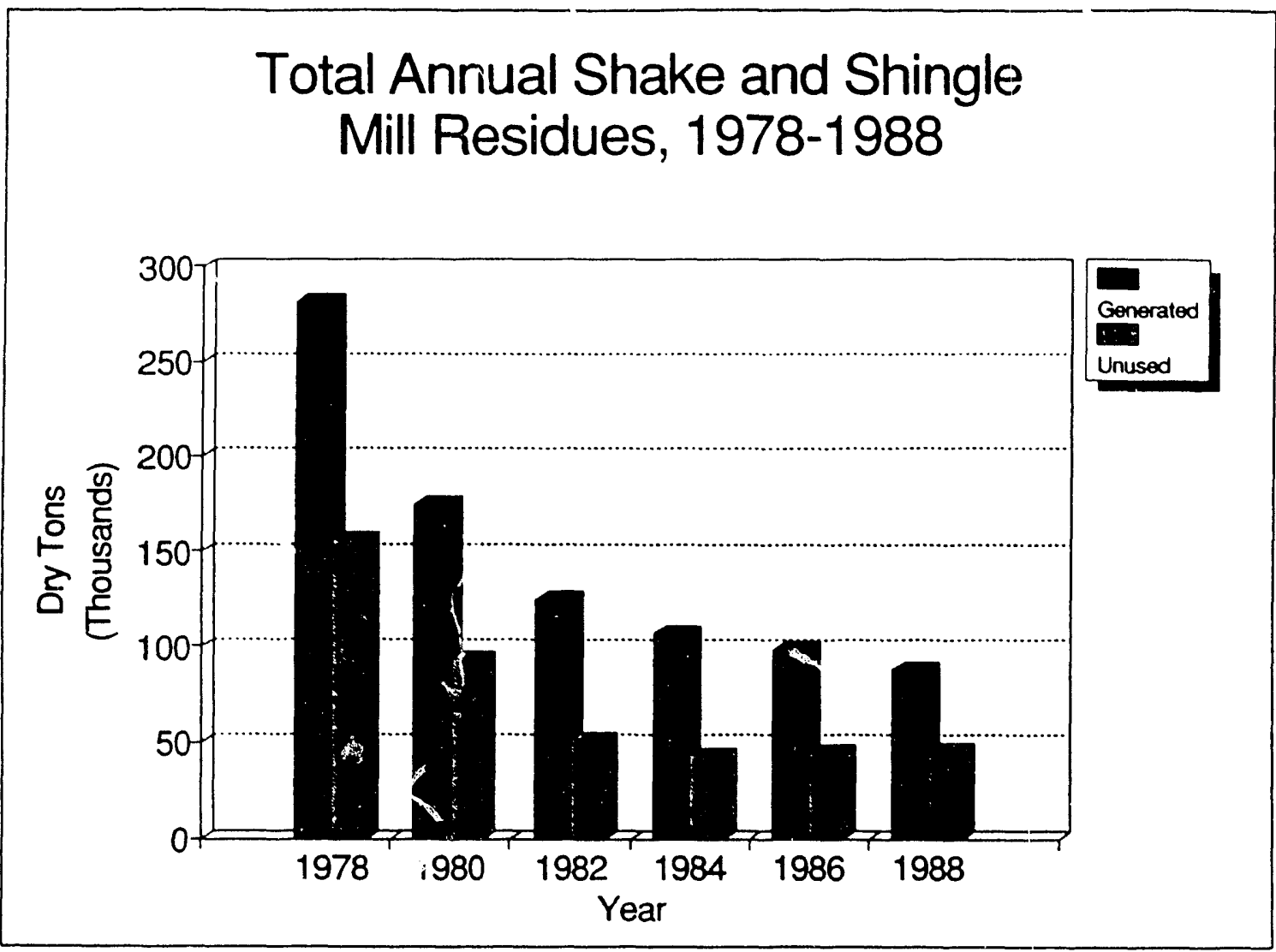

Figure l-15

Total Annual Generated \& Unused Veneer \& Plywood Mill Residues, 1978-1988

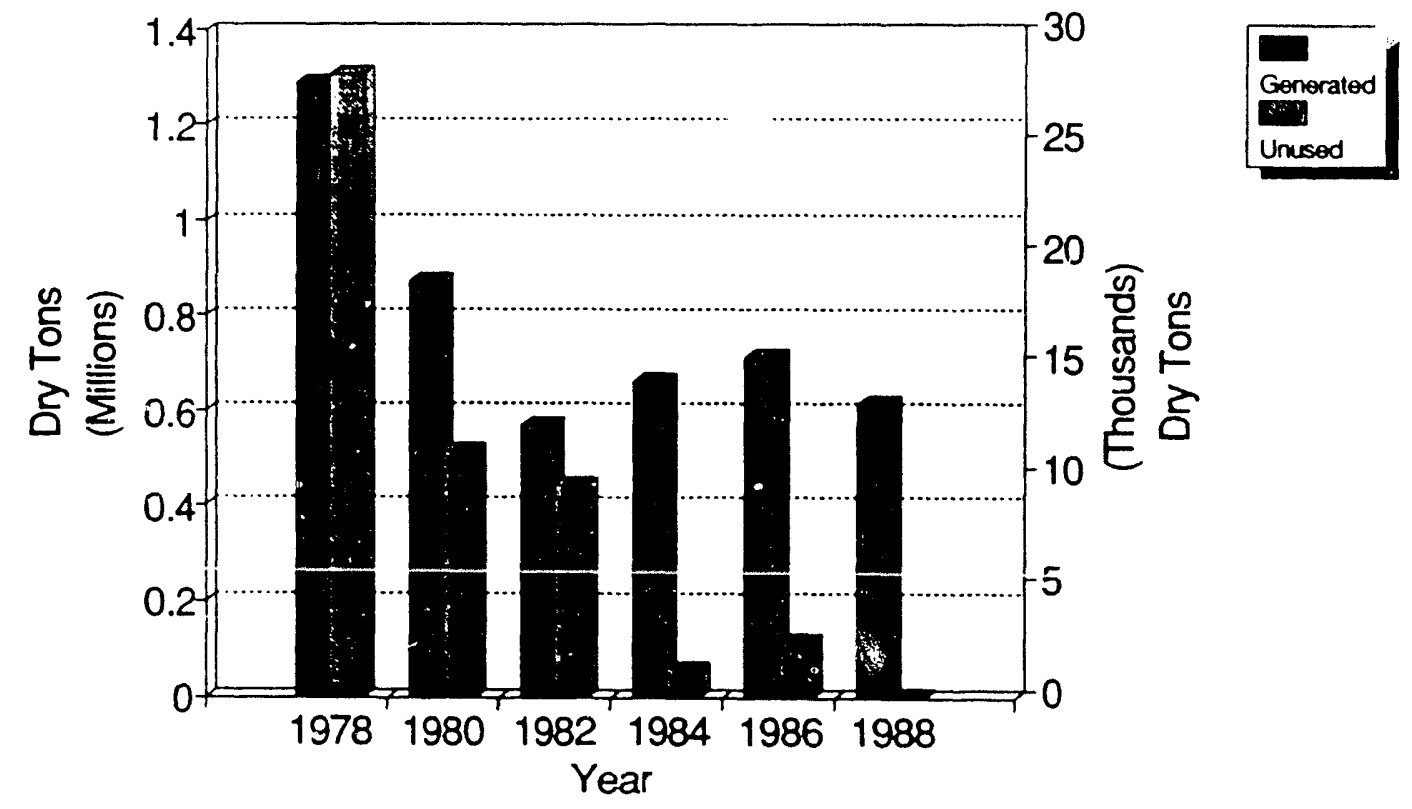




\section{Generated Mill Residues}

Annual Average

Ory ions (Thousands) By Region
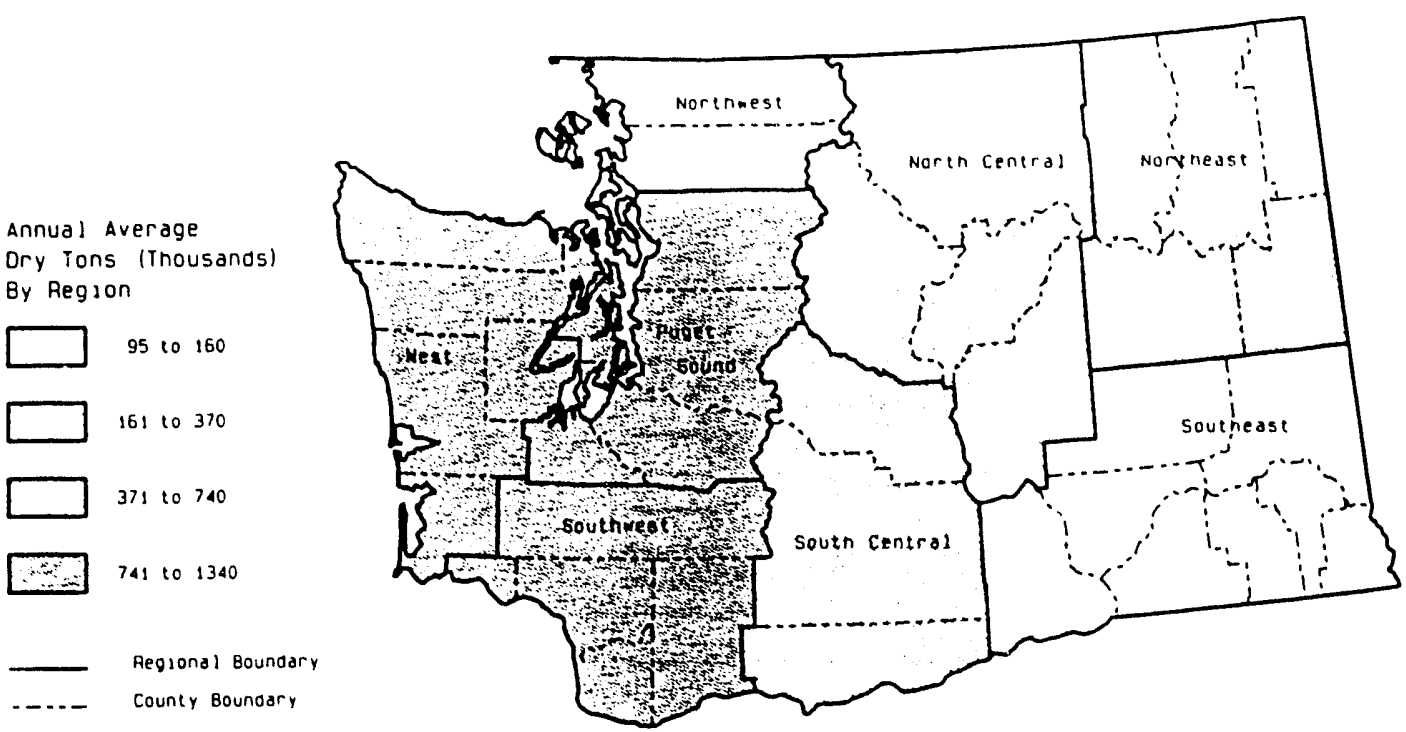

State of Washangton

Source. Wastington State Department of Natural Resources

Map 1-18

\section{Unused Mill Residues}

Annual Average

Dry Tons (Tnousand)

By Region

$\square$ (108

$\square 91020$

$\square 211052$

531080

- Hegional Boundary

-... Esuri, secosery

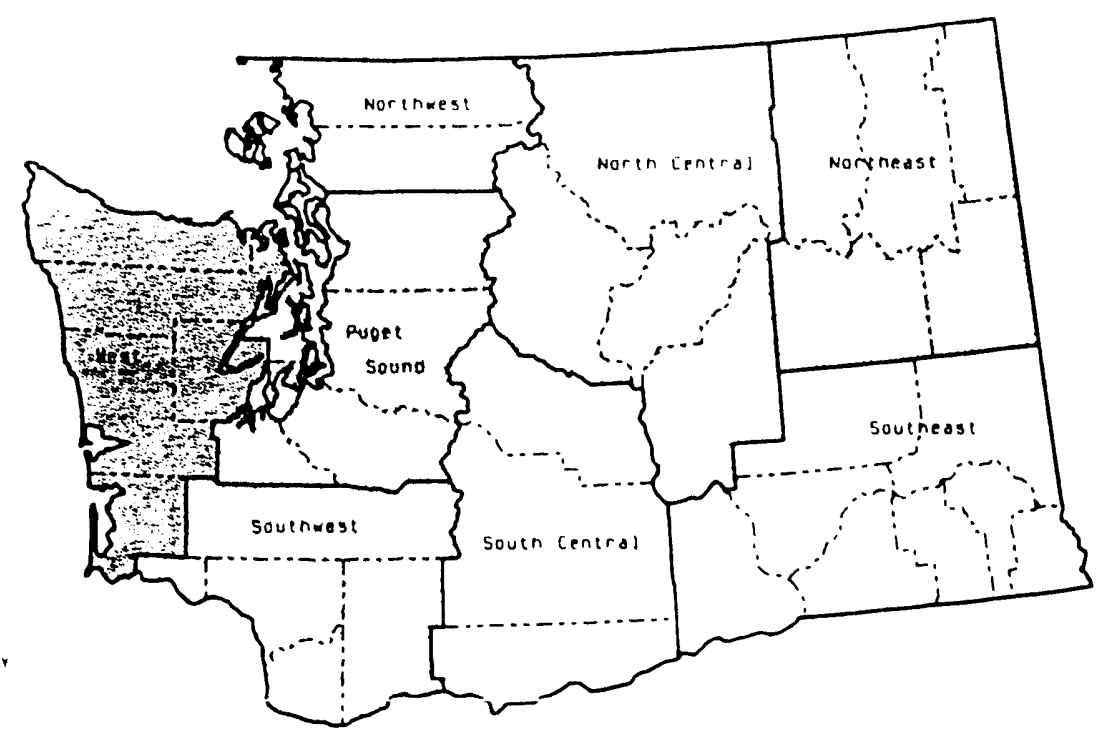

State of washington

Source wasnington state Degartaent of Natural Aesources 
Map 1-19

\section{Generated Sawmill Residues}

Annual Average

Ory Tons (Thousands)

By Aegion

$\square 0$ to 350

$\square$ 351 to 650

$\square 65110950$

951 to 1250

Regional Boundar
$\ldots \ldots$ County Bounoary

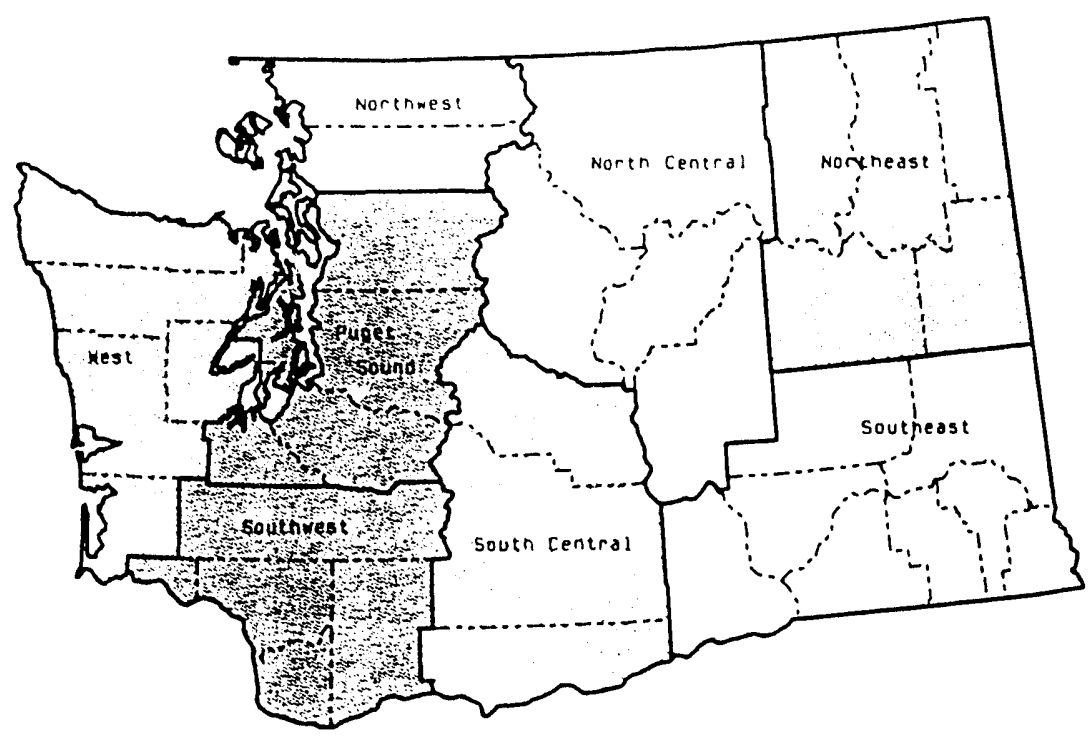

State of Washington

Source. Hashington Stale Depl. Of Natural Resources

Map 1-20

\section{Unused Sawmill Residues}

Annual Average

Jry Tons (Tnousanas)

By Region

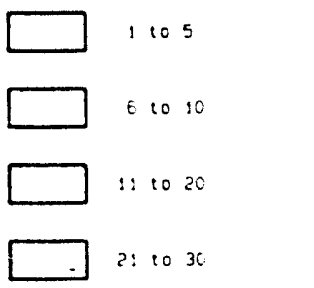

- Regional Boundary

-...- Councy Boundary

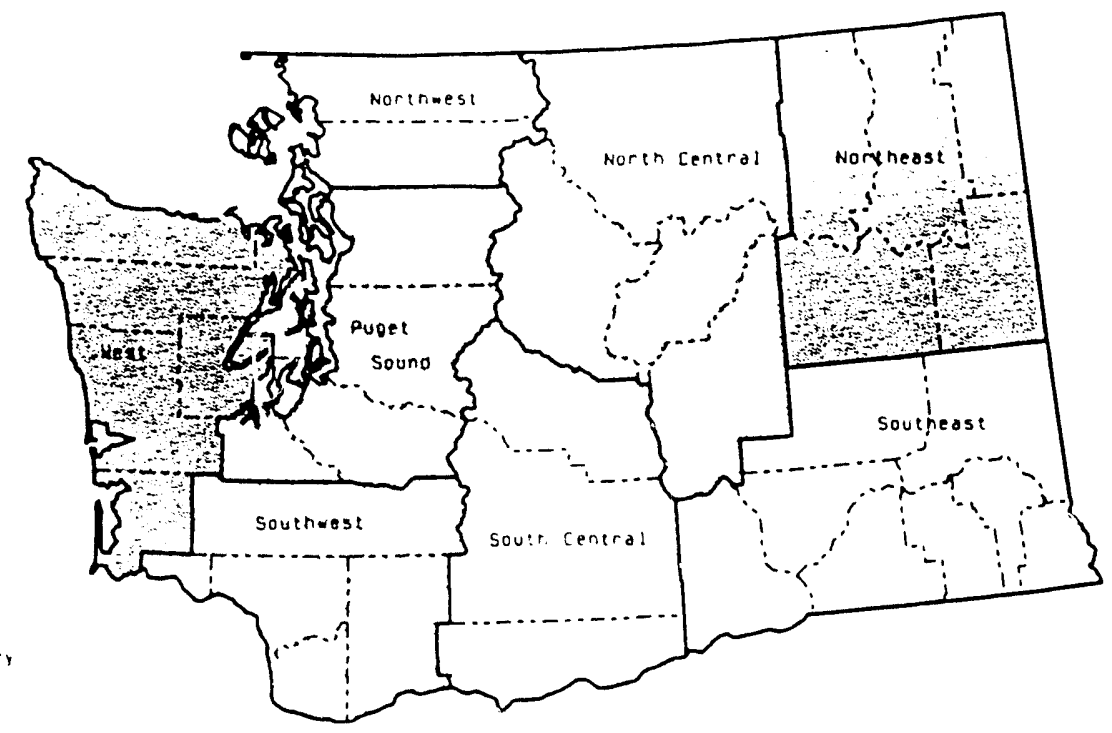

State of Washington

Source masnington State Dept of Natural Resources 


\section{Map I-21}

\section{Generated Shake and Shingle Mill Residues}

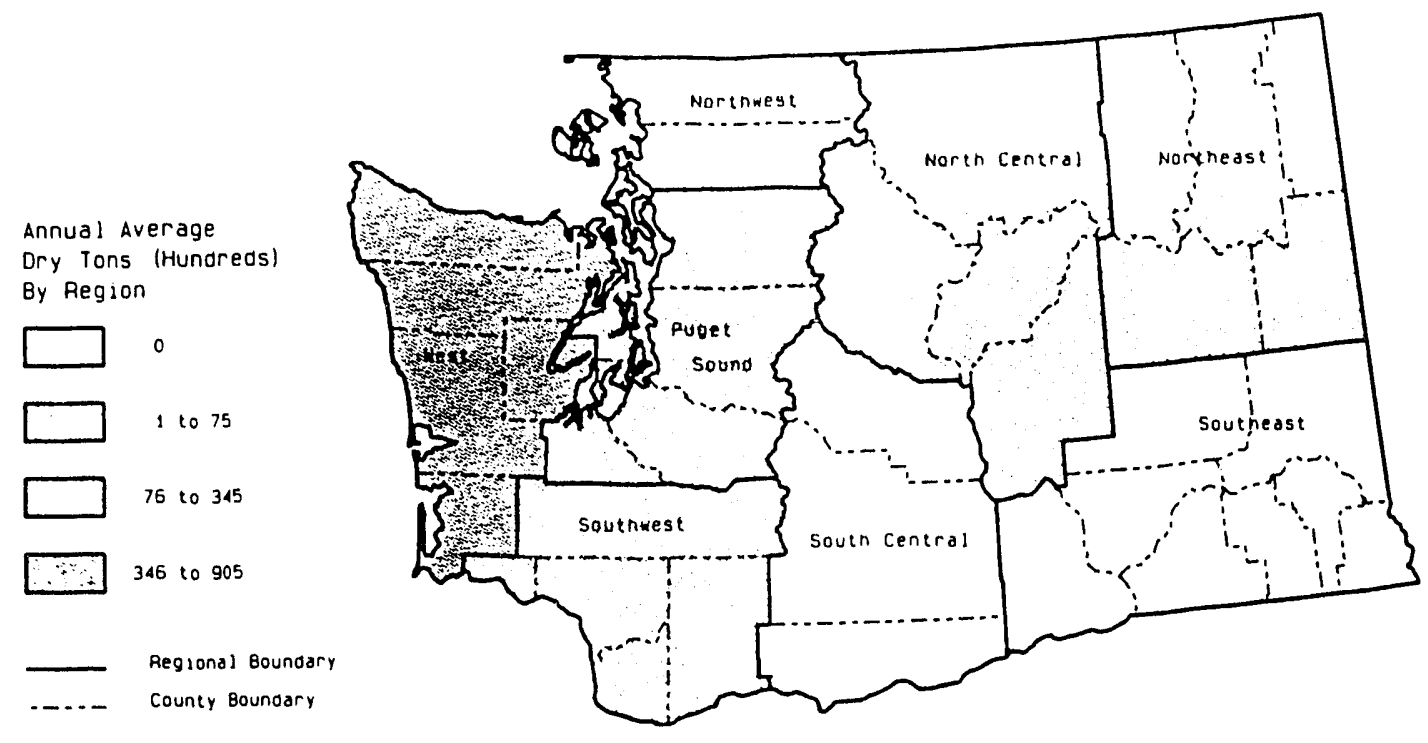

State of Washington

Source washangton State Dept. Of Natural Resources

\section{Map 1-22}

\section{Unused Shake and Shingle Mill Residues}

Annua! Average

Ory Tons (Hundreds) By Region
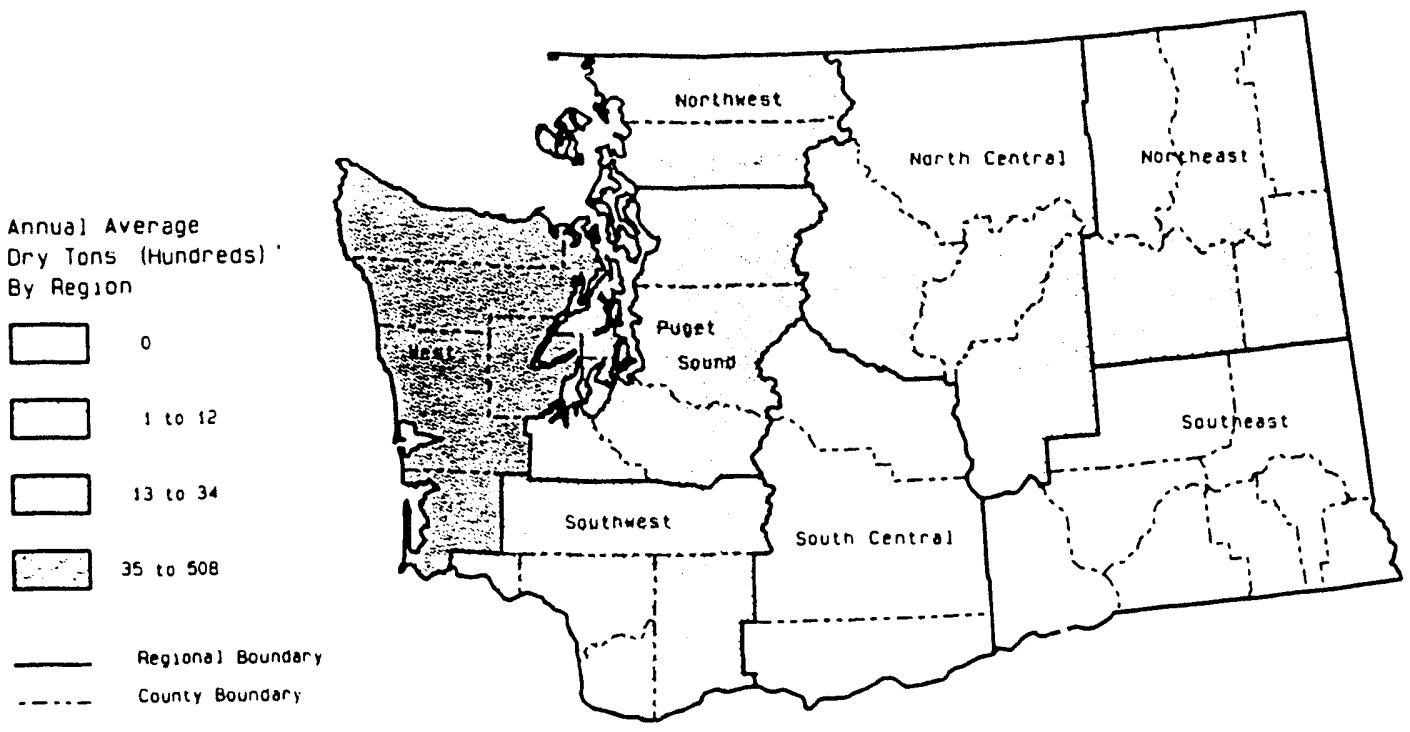

State of Washington

Source wasnington Szate Dept. Of Natural Resources 
Map 1-23

\section{Generated Veneer \& Plywood Residues}

Annual Average

Dry Tons (Inousanas)

By Region
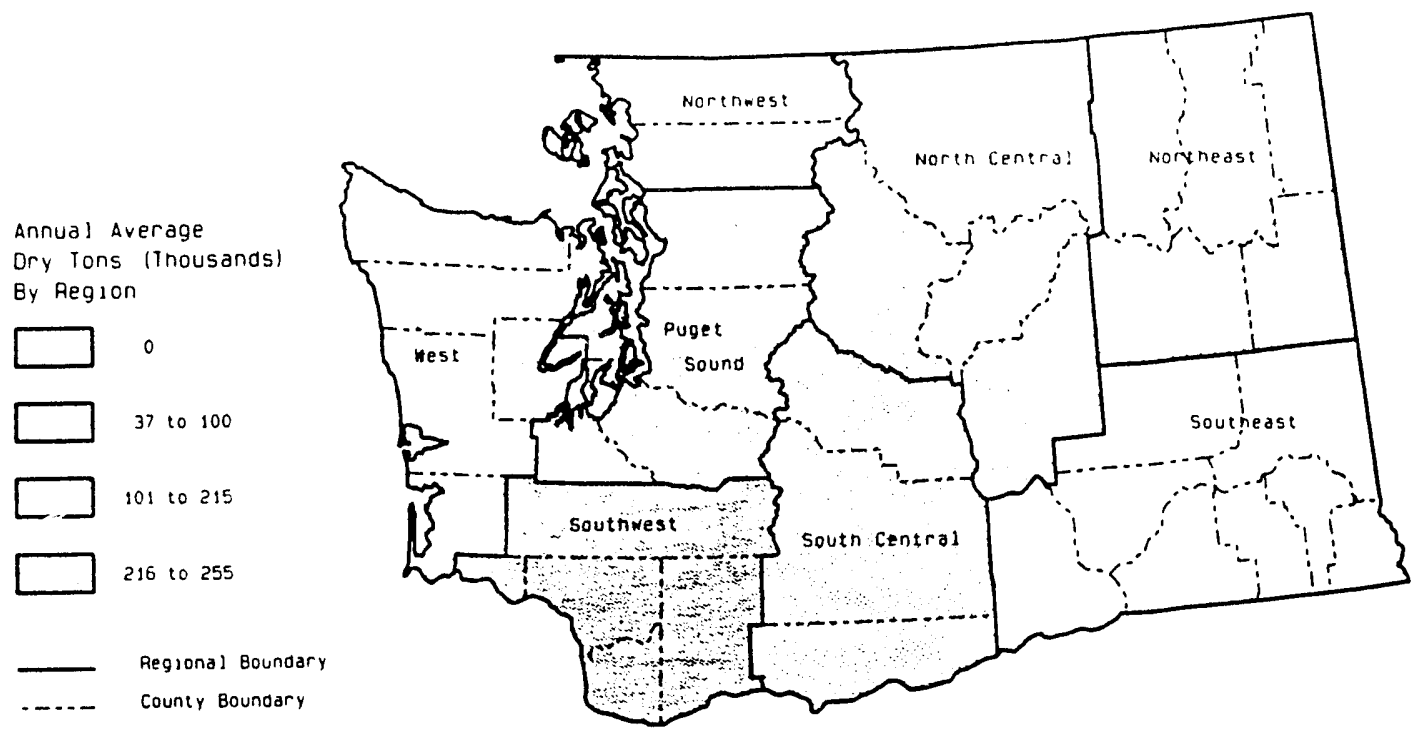

State of Washington

Source Masningtion State Deg: of Natural Resources

Map 1-24

\section{Unused Veneer \& Plywood Mill Residues}

Annual Average

Dry Tons (Hundreds)

By Region

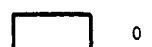

$\square$ प 510

11 to 20

21 1025

Regional Boundar

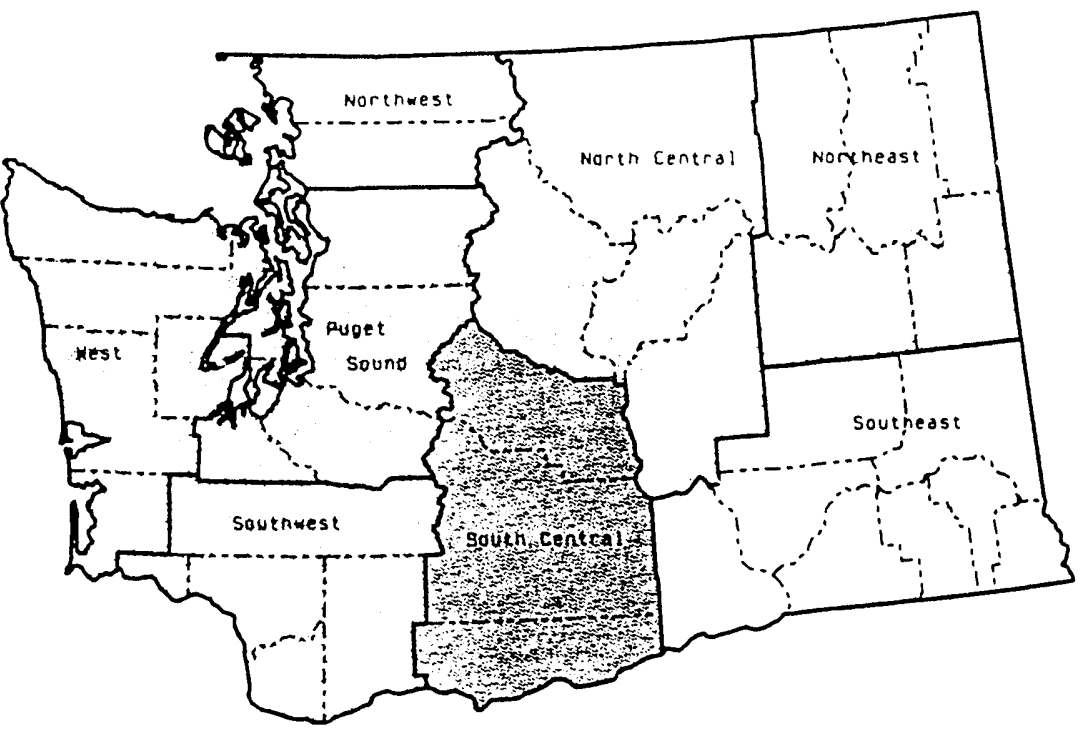

State of Washington

Source. Mashington State Dept of Natural Resources 
Table 1-38

Generated Mili Residues by Region

Sorted by Generated Annual Average (Dry Tons)

\begin{tabular}{|c|c|c|c|c|c|}
\hline \multirow[b]{2}{*}{ Rank } & \multirow[b]{2}{*}{ Region } & \multicolumn{2}{|c|}{--Generated Annual Average- } & \multicolumn{2}{|c|}{ Range-__L } \\
\hline & & (Dry Tons) & (MBtu) & Minimum & Maximum \\
\hline 1 & Southwest & $1,336,060$ & $24,049,074$ & $1,116,313$ & $1,582,973$ \\
\hline $\begin{array}{l}2 \\
3\end{array}$ & $\begin{array}{l}\text { Puget Sound } \\
\text { West }\end{array}$ & $\begin{array}{r}1,259,242 \\
926,388\end{array}$ & $\begin{array}{l}22,666,347 \\
16,674,990\end{array}$ & $\begin{array}{r}1,015,229 \\
828,141\end{array}$ & $\begin{array}{l}1,499,588 \\
1,085,014\end{array}$ \\
\hline $\begin{array}{l}4 \\
5\end{array}$ & $\begin{array}{l}\text { South Central } \\
\text { Northeast }\end{array}$ & $\begin{array}{l}556,316 \\
445,084\end{array}$ & $\begin{array}{r}10,013,696 \\
8,011,512\end{array}$ & $\begin{array}{l}403,111 \\
210,343\end{array}$ & $\begin{array}{l}690,173 \\
672,798\end{array}$ \\
\hline G & Northwest & 294,881 & $5,307,861$ & 127,522 & 429,696 \\
\hline 7 & North Central & 229,102 & $4,123,830$ & 172,377 & 269,686 \\
\hline 8 & Southeast & 94,617 & $1,703,106$ & 60,963 & 137,478 \\
\hline & State & $5,141,690$ & $92,550,416$ & $4,053,010$ & $5,953,643$ \\
\hline
\end{tabular}

Source: Wasi ..ngton State Department of Natural Resources, "Washington Mill Surveys," 1978-1988.

Table 1-39

Unused Mill Residues by Region

Sorted by Unused Annual Average (Dry Tons)

\begin{tabular}{|c|c|c|c|c|c|}
\hline \multirow[b]{2}{*}{ Rank } & \multirow[b]{2}{*}{ Region } & \multicolumn{2}{|c|}{ _-Unused Annual Average } & \multicolumn{2}{|c|}{-Range } \\
\hline & & (Dry Tons) & (MBtu) & Minimum & Maximum \\
\hline 1 & West & 77,546 & $1,395,828$ & 42,014 & 135,086 \\
\hline 2 & Northeast & 27,337 & 492,066 & 590 & 102,658 \\
\hline 3 & Puget Sound & 26,051 & 468,912 & 5,035 & 47,389 \\
\hline 4 & North Central & 16,278 & 293,010 & 5,747 & 26,220 \\
\hline 5 & Northwest & 15,212 & 273,810 & 3,607 & 29,243 \\
\hline 6 & Southwest & 13,635 & 245,427 & 1,145 & 49,829 \\
\hline 7 & Southeast & 3,701 & 66,618 & 0 & 22,162 \\
\hline 8 & South Central & 2,831 & 50,958 & 0 & 7,759 \\
\hline & State & 182,591 & $3,286,629$ & 114,173 & 409,848 \\
\hline
\end{tabular}

Source: Washington State Department of Natural Resources, "Washington Mill Surveys," 1978-1988. 
Table 1-40

Generated Sawmill Residues by Region

Sorted by Generated Annual Average (Dry Tons)

\begin{tabular}{|c|c|c|c|c|c|}
\hline \multirow[b]{2}{*}{ Rank } & \multirow[b]{2}{*}{ Region } & \multicolumn{2}{|c|}{-Generated Annual Average- } & \multirow{2}{*}{ Minimum } & \multirow{2}{*}{$\begin{array}{l}\text { Maximum } \\
\text { Maximate }\end{array}$} \\
\hline & & (Dry Tons) & (MBtu) & & \\
\hline & Puget Sound & $1,105,370$ & $19,896,651$ & 895,532 & $1,410,932$ \\
\hline & $\begin{array}{l}\text { Southwest } \\
\text { West }\end{array}$ & $\begin{array}{r}1,069,104 \\
766,600\end{array}$ & $\begin{array}{l}19,243,866 \\
13,798,800\end{array}$ & $\begin{array}{l}848,528 \\
696,762\end{array}$ & $\begin{array}{r}1,383,397 \\
851,833\end{array}$ \\
\hline & $\begin{array}{l}\text { Northeast } \\
\text { South Central }\end{array}$ & $\begin{array}{l}395,643 \\
377,997\end{array}$ & $\begin{array}{l}7,121,571 \\
6,803,946\end{array}$ & $\begin{array}{l}184,614 \\
297,291\end{array}$ & $\begin{array}{l}620,488 \\
486,271\end{array}$ \\
\hline & $\begin{array}{l}\text { Norttwest } \\
\text { North Central }\end{array}$ & $\begin{array}{l}209,219 \\
191,387\end{array}$ & $\begin{array}{l}3,765,936 \\
3,444,960\end{array}$ & $\begin{array}{r}56,838 \\
152,993\end{array}$ & $\begin{array}{l}353,951 \\
224,322\end{array}$ \\
\hline & 3 Southeast & 94,617 & $1,703,106$ & 90,932 & 38,801 \\
\hline & State & $4,209,935$ & $75,778,836$ & $3,358,266$ & $5,247,504$ \\
\hline
\end{tabular}

Source: Washington State Department of Natural Resources, "Washington Mill Survey," 1978-1988.

Table $1-41$

\section{Unused Sawmill Residues by Region}

Sorted by Unused Annual Average (Dry Tons)

\begin{tabular}{|c|c|c|c|c|c|}
\hline \multirow[b]{2}{*}{ Rank } & \multirow[b]{2}{*}{ Region } & \multicolumn{2}{|c|}{--Unused Annual Average } & \multicolumn{2}{|c|}{ Range-_-_ } \\
\hline & & (Dry Tons) & (MBtu) & Minimum & Maximum \\
\hline 1 & Northeast & 26,468 & 476,424 & 590 & 100,212 \\
\hline & 2 West & 25,332 & 455,970 & 8,184 & 59,833 \\
\hline & 3 Puget Sound & 18,868 & $339,1 j 18$ & 648 & 45,834 \\
\hline 4 & 4 North Central & 15,428 & 277,704 & 5,732 & $26,2 ? 0$ \\
\hline 5 & 5 Southwest & 9,083 & 163,500 & 660 & 34,825 \\
\hline 6 & 6 Southeast & 3,701 & 66,618 & 0 & 22,162 \\
\hline 7 & 7 Norttwwest & 1,951 & 35,121 & 129 & 3,451 \\
\hline 8 & 8 South Central & 533 & 9,597 & 0 & 1,367 \\
\hline & State & 101,364 & $1,824,552$ & 54,619 & 226,059 \\
\hline
\end{tabular}

Source: Washington State Department of Natural Resources, "Washington Mill Survey," 1978-1988. 
Table 1-42

Generated Shake and Shingle Mill Residues By Region

Sorted by Generated Annual Average (Dry Tons)

$\begin{array}{ll}\text { Rank Region } & \text {-Generated Annual Average- } \\ \text { (Dry Tons) } & \text { (MBtu) }\end{array}$

Minimum Range- Maximum

\begin{tabular}{llrrr}
\hline West & 90,104 & $1,621,878$ & 55,148 & 180,614 \\
2 Northwest & 20,596 & 370,719 & 6,665 & 33,171 \\
3 Southwest & 17,576 & 316,365 & 1,703 & 37,472 \\
4 Puget Sound & 15,249 & 274,482 & 6,858 & 26,858 \\
5 Northeast & 363 & 6,537 & 0 & 1,562 \\
6 North Central & 322 & 0 & 1,710 & 1,710 \\
7 Southeast & 0 & 0 & 0 & 0 \\
8 South Central & 0 & 0 & 0 & 0 \\
& & & & 281,387
\end{tabular}

Source: Washington State Department of Natural Resources, "Washington Mill Survey," 1978-1988.

Table 1-43

Unused Shake and Shingle Mill Residues By Region

Sorted by Unused Annual Average (Dry Tons)

Rank Region

-Unused Annual Average-

(

(Dry Tons)

(MBtu)

Minimum Range-

$\begin{array}{ll}1 & \text { West } \\ 2 & \text { Northwest } \\ 3 & \text { Puget Sound } \\ 4 & \text { Southwest } \\ 5 & \text { North Central } \\ 6 & \text { Northeast } \\ 7 & \text { Southeast } \\ 8 & \text { South Central }\end{array}$

50,826

12,789

914,874

230,193

5,813

104,634

2,708

48,735

4,884

271

1,956

0

0

0

0

28,84
3,478
547
485

101,648

25,073

19,908

$\begin{array}{rr}7,219 \\ 0 & 1,563\end{array}$

$0 \quad 336$

State

72.515

$1.305,276$

43.566

155,747

Source: Washington State Department of Natural Resources, "Washington Mill Survey," 1978-1988. 
Table 1-44

Generated Veneer \& Plywood Mill Residues By Region

Sorted by Generated Annual Average (Dry Tons)

\begin{tabular}{|c|c|c|c|c|c|}
\hline \multirow[b]{2}{*}{ Re, ik } & \multirow[b]{2}{*}{ Region } & \multicolumn{2}{|r|}{ verage- } & \multicolumn{2}{|c|}{-Range } \\
\hline & & (Dry Tons) & (MBtu) & Minimum & Maximum \\
\hline 1 & Soutthwest & 251,707 & $4,530,728$ & 180,606 & 489,416 \\
\hline $\begin{array}{l}2 \\
3\end{array}$ & $\begin{array}{l}\text { South Central } \\
\text { Puget Sound }\end{array}$ & $\begin{array}{l}178,319 \\
136,296\end{array}$ & $\begin{array}{r}105,820 \\
2,453,336\end{array}$ & $\begin{array}{r}235,084 \\
68,866\end{array}$ & $\begin{array}{l}277,453 \\
238,681\end{array}$ \\
\hline 4 & West & 69,684 & $1,254,318$ & 45,519 & 111,461 \\
\hline 5 & Northwest & 65,067 & $1,171,214$ & 26,335 & 116,747 \\
\hline 6 & Northeast & 49,078 & 883,412 & 25,442 & 61,032 \\
\hline 7 & North Central & 37,393 & 673,076 & 19,384 & 46,500 \\
\hline 8 & Southeast & 0 & 0 & 0 & 0 \\
\hline & State & 787,546 & $11,071,905$ & 569,503 & $1,290,544$ \\
\hline
\end{tabular}

Sources: Washington State Department of Natural Resources, "Washington Mill Survey," 1978-1988; 1982 Directory of the Forest Products Industry

Table $1-45$

Unused Veneer \& Plywood Mill Residues By Region

Sorted by Unused Annual Average (Dry Tons)

\begin{tabular}{|c|c|c|c|c|c|}
\hline \multirow[b]{2}{*}{ Rank } & \multirow[b]{2}{*}{ Region } & \multicolumn{2}{|c|}{-Unused Annual Average_ } & \multicolumn{2}{|c|}{-Rango- } \\
\hline & & (Dry Tons) & (MBtu) & Minimum & Maximum \\
\hline 1 & South Central & 2,297 & 41,341 & 0 & $\overline{6,422}$ \\
\hline 2 & Southwest & 1,844 & 33,197 & 0 & 7,785 \\
\hline 3 & West & 1,388 & 24,975 & 0 & 5,743 \\
\hline 4 & Puget Sound & 1,370 & 24,653 & 0 & 3,656 \\
\hline 5 & Northeast & 760 & 13,687 & 0 & 2,110 \\
\hline 6 & North Central & 579 & 10,428 & 0 & 1,607 \\
\hline 7 & Northwest & 472 & 8,491 & 0 & 1,073 \\
\hline 8 & Southeast & 0 & 0 & 0 & 0 \\
\hline & State & 8,710 & 156,771 & 0 & 28,042 \\
\hline
\end{tabular}

1 WSEO Estimate

Sources: Washington State Department of Natural Resources, "Washington Mill Survey," 1978-1988; 1982 Directory of the Forest Products Industry 
Table 1-46

Mill Residues Generated and Amount Available for Cost of Transportation

Generated

Available

$1984-1985$

1991-1995

$1996-2000$

$2001-2010$

Generated

100.10

Available High

8.80

104.4

11.6

107.1

128.5

Available Low

$-0.51$

1.5

13.3

26.2

13.9

Source: Washington State Energy Office 


\section{Secondary Wood Processing Residues}

Maps and Tables 


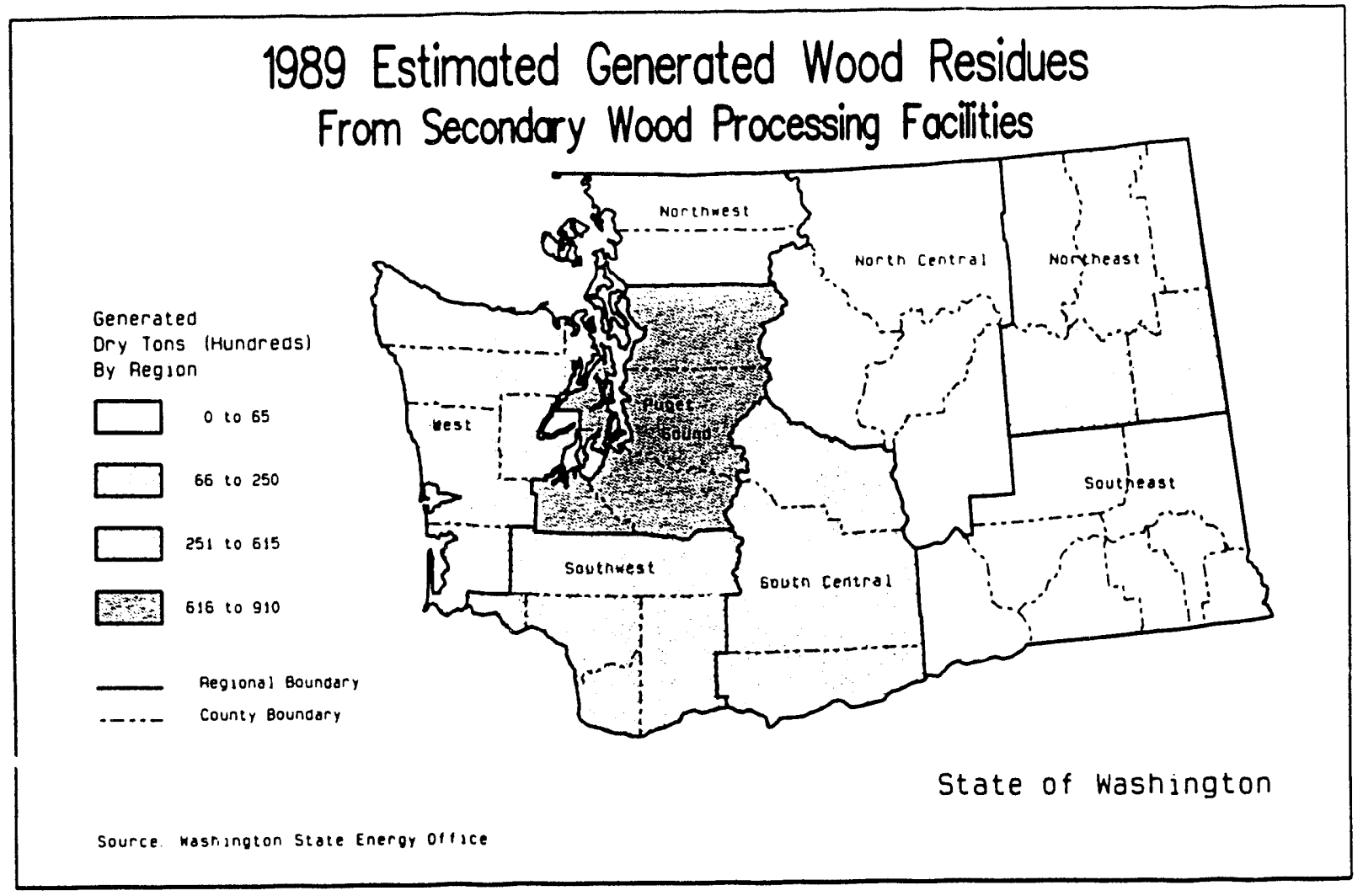

Map 1-26

\section{Estimated Unused Wood Residues From Secondary Wood Processing Faciities}

Unused

Dry Tons (Hunoreas) By Region

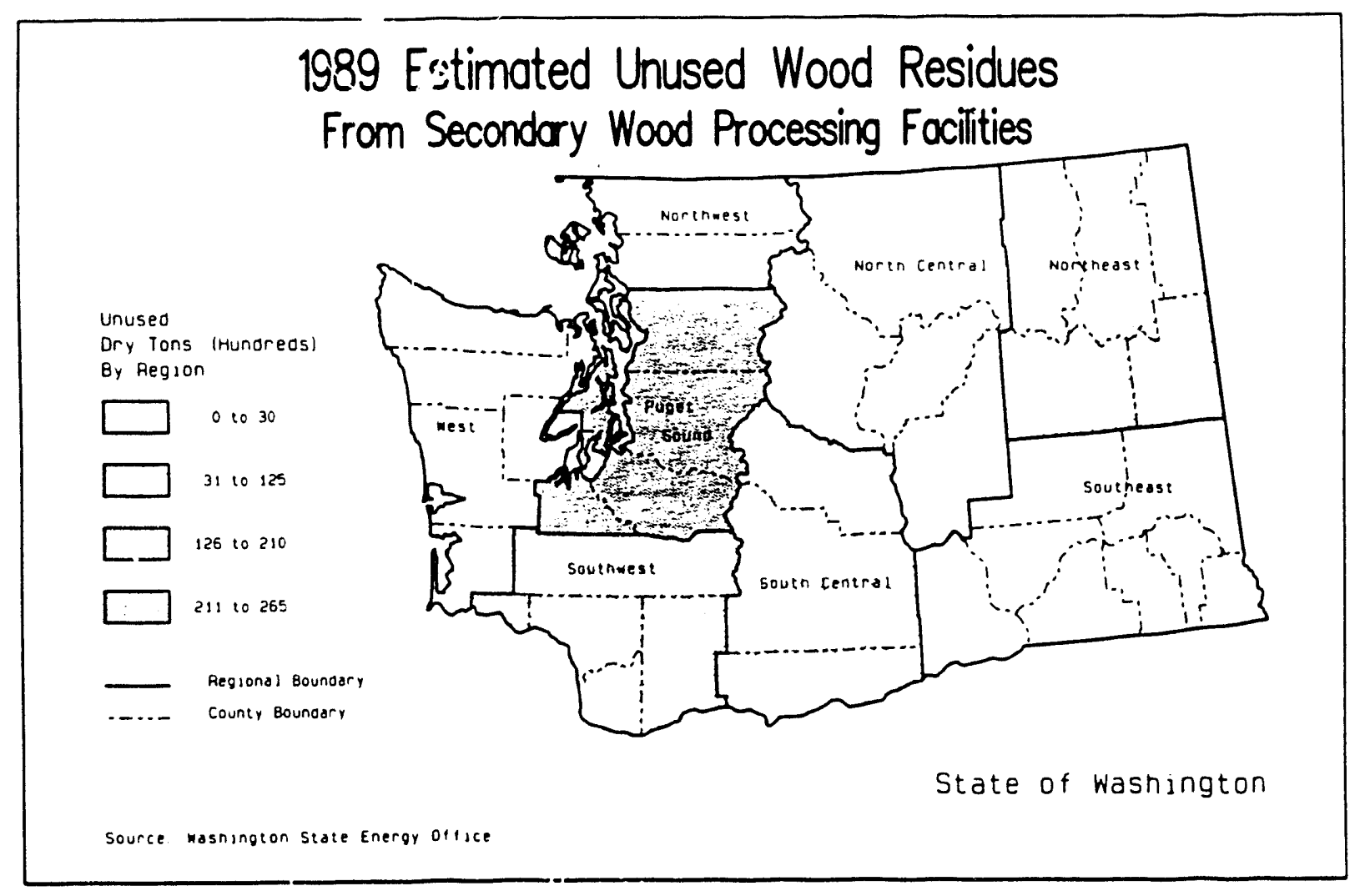


Table $1-47$

\section{Generated Secondary Wood Processing Residues by Region}

Ranked by Generated Dry Tons

\begin{tabular}{llrr}
\hline Rank & Kregion & $\begin{array}{c}\text { Generated1 } \\
\text { (Dry Tons) }\end{array}$ & $\begin{array}{c}\text { Energy Equivalent }{ }^{2} \\
\text { (MBtu) }\end{array}$ \\
\hline 1 & Puget Sound & 90,767 & $1,633,815$ \\
2 & Southwest & 32,198 & 579,573 \\
3 & Northeast & 17,517 & 315,297 \\
4 & West & 16,354 & 294,348 \\
5 & South Central & 9,736 & 175,249 \\
6 & Southeast & 3,291 & 59,218 \\
7 & Northwest & 31 & 562 \\
8 & North Central & 5 & 63 \\
& State Total & 169,899 & $3,058,126$ \\
1 & &
\end{tabular}

Table 1-48

\section{Unused Secondary Wood Processing Residues by Region}

Ranked by Unused Residues (Dry Tons)

\begin{tabular}{llcc}
\hline Region & $\begin{array}{c}\text { Unused Residue } \\
\text { (Dry Tons) }\end{array}$ & $\begin{array}{c}\text { Energy Equivalent }{ }^{2} \\
\text { (M Btu) }\end{array}$ \\
\hline 1 & Puget Sound & 26,291 & $473,24,7$ \\
2 & Southwest & 15,435 & 277,828 \\
3 & South Central & 9,589 & 172,595 \\
4 & Northeast & 6,880 & 123,835 \\
5 & Northwest & 22 & 403 \\
6 & Southeast & 7 & 117 \\
7 & West & 6 & 114 \\
8 & North Central & 1 & 20 \\
& State Total & 58,231 & $1.048,159$ \\
& &
\end{tabular}


Table $1-49$

Generated Secondary Wood Processing Residues by County

Ranked by Generated Dry Tons

Rank County (Region)

Generated*

Energy Equivalent**

(Dry Tons)

(M Btu)

1 Pierce (PS)

36,363

654,535

2 Snohomish (PS)

36,108

3 Cowlitz (SW)

25,003

4 King (PS)

5 Spokane (NE)

18,115

17,514

6 Grays Harbor (W)

16,332

7 Yakimia (SC)

9,731

8 Lewis (SW)

4,001

3,283

3,124

10 Clark (SW)

11 Thurston (PS)

169

649,942

12 Wahkiakum (SW)

70

450,059

13 Clallam (W)

20

326,069

14 San Juan (NW)

$15 \mathrm{Kitsap}$ (PS)

16 Whatcom (NW)

17 Benton (SE)

18 Kittitas (SC)

19 Island (NW)

20 Chelan (NC)

21 Stevens (NE)

22 Skagit (NW)

23 Jefferson (W)

24 Whitman (SE)

25 Grant (NC)

26 Adams (SE)

27 Mason (W)

28 Franklin (SE)

29 Okanogan (NC)

30 Douglas (NC)

31 Pend Oreille (NE)

32 Lincoln (NE)

33 Garfield (SE)

34 Skamania (SW)

35 Pacific (W)

36 Asotin (SE)

37 Ferry (NE)

38 Kickitat (SC)

39 Walla Walla (SE)

315,257

293,977

175,159

72,016

59,094

56,238

3,035

1,260

359

353

234

130

112

90

66

58

41

14

10

9

0

$>1$

$>1$

$>1$

$>1$

NR

NR

NR

NR

NR

NR

NR

NR

NR

NR

NR

0

0

0

NR

NR

NR

NR

NR

NR

NR

NR

NR

NR

NR

State Total

169,899

$3,058,116$

"Reported unused residues. $\quad$ "* Energy equivalent, $18 \mathrm{M}$ Btuton. $\quad$ NR - Not Reported.

Source: Washington State Energy Office. "1900 Wood Residue Survey and Directory of Secondary Wood Processing Fadities In Washington State." 
Table $1-50$

Unused Secondary Wood Processing Residues by County

Ranked by Unused Dry Tons

Rank County (Region)

Unused*

Energy Equivalent**

(Dry 'Tons)

(M Btu)

1 King (PS)

2 Cowitz (SW)

3 Yakima (SC)

4 Spokane (NE)

5 Pierce (PS)

6 Snohomish (PS)

7 Lewis (SW)

8 Clark (SW)

9 Thurston (PS)

10 Wahkiakum (SW)

11 San Juan (NW)

12 Kitsap (PS)

13 Benton (SE)

14 Clallam (W)

15 Kittitas (SC)

16 Island (NW)

17 Whatcom (NW)

18 Chelan (NC)

19 Jefferson (W)

20 Grant (NC)

21 Stevens (NE)

22 Adams (SE)

23 Whitman (SE)

24 Mason (W)

25 Franklin (SE)

26 Columbia (SE)

27 Grays Harbor (W)

28 Skagh (NW)

NA Asotin (SE)

NA Douglas (NC)

NA Ferry (NE)

NA Garfield (SE)

NA Klickitat (SC)

NA Lincoln (NE)

NA Okanogan (NC)

NA Pacific (W)

NA Pend Oreille (NE)

NA Skamania (SW)

NA Walla Walla (SE)

State Total
17,367

12,503

9,584

6,879

5,192

3,621

2,001

861

100

70

19

13

6

6

5

2

1

1

0.48

0.28

0.25

0.13

0.13

0.10

0.04

0.00

0.00

0.00

NR

NR

NR

NR

NR

NR

NR

NR

NR

NR

NR

58,231

- Reported unused residues. $\quad$ "Energy equivalent, $18 \mathrm{M}$ Btution. NR - Not Reported.

312,599

225,053

172,506

123,830

93,451

65,170

36,012

15,503

1,794

1,260

350

233

112

104

90

36

17

15

9

5

5

2

2

2

1

0

0

0

NR

NR

NR

NR

NR

NR

NR

NR

NR

NR

NR

$1,048,159$

Source: Washington State Energy Office. "1990 Wood Residue Survey and Directory of Secondary Wood Processing Facilities In Washington State." 


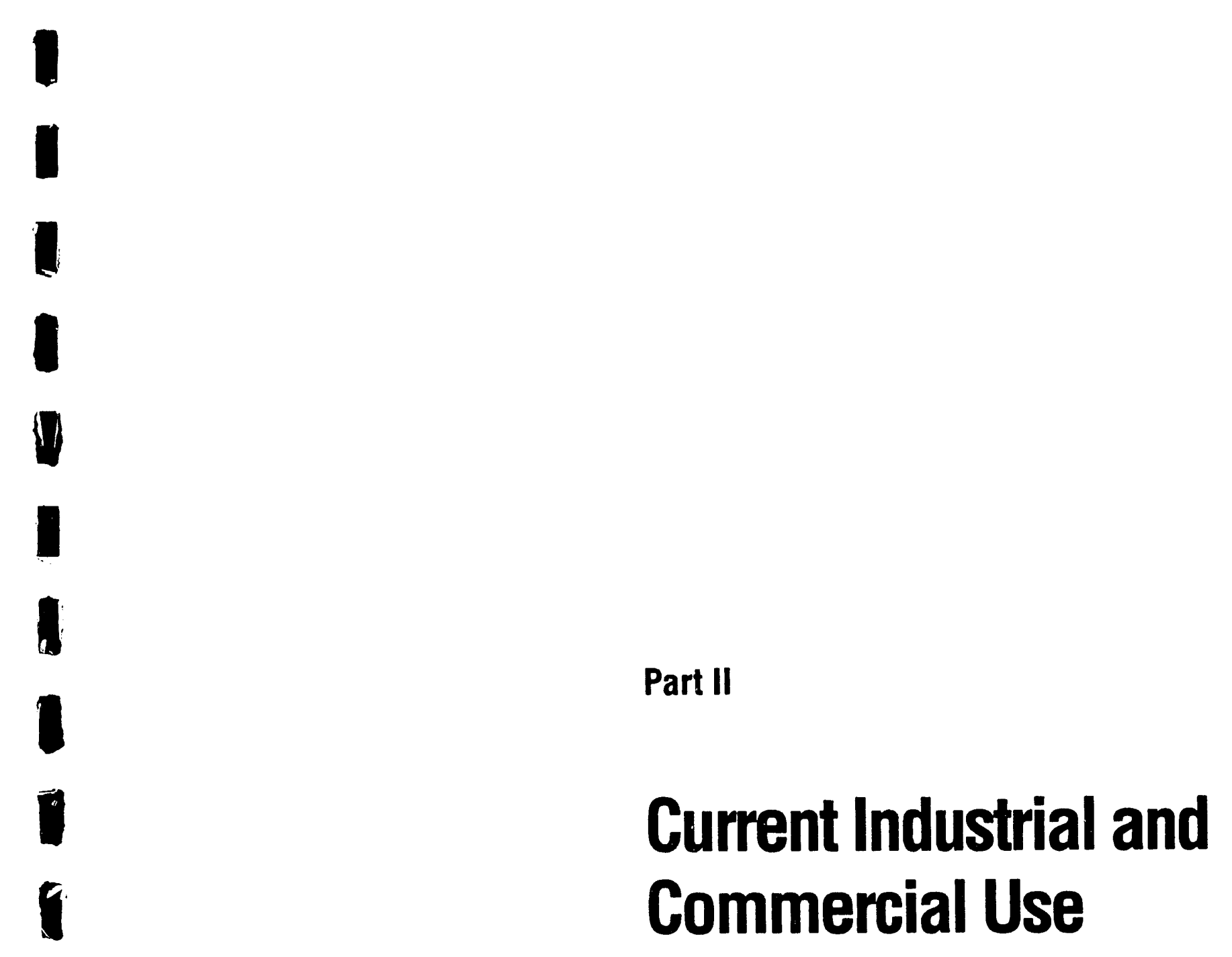




\section{Part II}

\section{Current Industrial and Commercial Use}

\section{Agricultural Residues}

\section{Biogas Use}

Currently in Washington state agricultural field crop residues are left on the ground for soil improvement and erosion control or converted to animal feed. The remaining residues are burned on-site. It is not known how much, if any, is being consumed for energy.

Residues can be combusted in bale burners and pellet/cube/log burners. Under more controlled environments, agricultural wastes can be converted to energy in combustors equipped with state-ofthe-art pollution control devices. Some problems associated with handling agricultural field residues are that residues tend to lose energy during storage, are only seasonally available, resist densification, and have a high ash content.

Residues can be processed into pellets, cubes, or straw logs through pulverizing and compressing them. Compressed or densified residues makes storage and handling easier. Agricultural field residues have an approximate heating value of 15 million Btu per ton.

In Washington state, biogas is primarily produced by sewage treatment facilities through a process known as anaerobic digestion. Sludge is placed in a digester for a selected length of time and allowed to convert to methane and carbon dioxide. Biogas has a heating value of approximately 600 British thermal units per standard cubic foot of gas. Commercial natural gas-a combination of methane, propane, and butane - has a net heating value of approximately $1,050 \mathrm{Btu}$.

The biogas is used in a variety of ways. For the most part, it is primarily burned in boilers to meet sewage treatment facilities' space and process heating needs. Larger biogas producers sell biogas as a boiler fuel; scrub and treat it to pipeline quality then sell it to the gas utility; or use it as vehicle fuel. Excess biogas is usually disposed of in a waste gas flare.

Table II-1, page II-4 shows sewage capacity of sewage treatment plants, average biogas output, and gross energy equivalent. 


\section{Municipal Solid Waste}

\section{Wood Wastes}

Rapid reduction in existing landfill space, restrictive landfill regulations, and the political and technical difficulties of siting new landfills provide good incentives to develop waste-to-energy projects in Washington state. Currently, there are three municipal solid waste combustion facilities in Washington state and all are operating at capacity (see Table II-2, page II-5). A fourth facility will open in Spokane in the fall of 1991. The combustible fraction of municipal solid waste has an approximate heating value of 14 million Btu per ton.

The Clean Air Act tightened municipal solid waste incinerator emission laws. However, the 1991 national energy strategy includes provisions to encourage the development of waste-to-energy projects. Waste-to-energy will be more widely used once environmental concerns are addressed.

Although there is an interest in the energy recovery potential from our landfills, developing new solid waste combustion facilities will be determined by the political, environmental, and economic costs of the various disposal alternatives and will be decided on a caseby-case basis.

Wood wastes annually contribute a significant amount towards energy consumption in Washington state, see pages II- 6 through II-8. Wood wastes are used for both steam generation and electric power cogeneration within the commercial and industrial sectors. Table II-3 shows energy consumption and gross energy equivalent of wood combustion facilities in the forest products industries. Wood consumption in the industrial sector accounts for approximately 30 percent of the total industrial energy use. Table II-4 shows state facilities that consume wood wastes and the gross energy equivalent of the amount consumed.

Washington Water Power operates the only private utility-owned, wood-fired electric power plant in Washington state that uses forest and mill residues to generate electricity. The $48 \mathrm{MW}$ plant is located in Kettle Falls in Stevens County (see Table II-5).

Table II- 6 shows the solid fuel processing facilities that use mill residues to produce fuel in the form of pellets and logs.

A certain percentage of forest residues left after commercial logging operations are normally available to woodcutters. These residues are primarily used by the residential sector as a primary or secondary source of heating fuel. The actual amount of wood taken from these forests is hard to document.

Mills and secondary wood industries have been effective at finding end uses for their waste. Mills use or sell over 96 percent of residues generated. Secondary wood processing facilities either sell or give away the majority of their sawdust and scraps. 
Environmental laws have limited the amount of old growth timber available to be harvested. Because shake and shingle mills depend primarily on the use of old growth western red cedar, this industry is expected to decline in the future (DNR, 1990).

Washington State Department of Natural Resources. Washington Mill Survey—1986. Pages D-14 \& 15. 1990. 
Table II-1

\section{Biogas Production Facilities}

Sorted by Average Biogas Output

\begin{tabular}{|c|c|c|c|c|}
\hline County (Region) & Facility & $\begin{array}{r}\text { Sewage } \\
\text { Capacity } \\
\text { (M Gal/Day) }\end{array}$ & $\begin{array}{l}\text { Avg. Biogas } \\
\text { Output } \\
\text { (M Cub.Ft. } / \text { r) }\end{array}$ & $\begin{array}{r}\text { Gross Energy } \\
\text { Equivalent } \\
(\mathrm{MBtu} / \mathrm{r})\end{array}$ \\
\hline King (PS) & Renton Treatment Plant* & 72 & 339 & 203,400 \\
\hline King (PS) & West Point Treatment Plant** & 125 & 256 & 153,600 \\
\hline Spokane (NE) & Spokane Wastewater Treatment Plant & 44 & 200 & 120,000 \\
\hline Pierce (PS) & Tacoma STP-1* & 28 & 80 & 48,000 \\
\hline Kitsap (PS) & Port Orchard/Kitsap County Sewer Dist. \#5 & 2.8 & 55 & 33,000 \\
\hline Thurston (PS) & LOTT** & 22 & 45 & 27,000 \\
\hline Pierce (PS) & Pierce County Chambers Creek Treatment & 12 & 32 & 19,200 \\
\hline Pierce (PS) & Puyallup Wastewater Treatment Plant & 10.7 & 30 & 18,000 \\
\hline Pend Oreille (NE) & Newport. City of & 0.5 & 30 & 18,000 \\
\hline Franklin (SE) & Pasco, City of & 4.25 & 24 & 14,400 \\
\hline King (PS) & Des Moines Creek Treatment Plant & 6 & 21 & 12,600 \\
\hline King (PS) & SW Suburban Sewer District - Salmon Cre & 5.8 & 15 & 9,000 \\
\hline King (PS) & SW Suburban Sewer District - Miller Creek & 4.7 & 15 & 9,000 \\
\hline King (PS) & METRO/Alki Treatment Plant & 15 & 14 & 9,000 \\
\hline Walla Walla (SE) & Walla Walla Wastewater Treatment Plant & 15 & 14 & 8,400 \\
\hline Kitsap (PS) & Bremerton, City of** & 15 & 14 & 8,400 \\
\hline Lewis (SW) & Chehalis, City of & 2 & 13 & 7,800 \\
\hline Kitsap (PS) & Central Kitsap Treatment Plant & 4.8 & 9 & 5,400 \\
\hline Kittitas (SC) & Ellensburg, City of & 15 & 8 & 4,800 \\
\hline King (PS) & Federal Way Water \& Sewer & 13.8 & 8 & 4,800 \\
\hline Chelan (NC) & Wenatchee Water Pollution Control Plant & 5 & 8 & 4,800 \\
\hline Lewis (SW) & Centralia, City of** & 4.3 & 8 & 4,800 \\
\hline Skagit (NW) & Anacortes, City of & 2.2 & 8 & 4,800 \\
\hline Yakima (SC) & Sunnyside Wastewater Treatment Plant & 2.18 & 8 & 4,800 \\
\hline Douglas (NC) & Douglas County Sewer District & 1.6 & 8 & 4,800 \\
\hline Benton (SE) & Prosser, City of & 1.5 & 8 & 4,800 \\
\hline Yakima (SC) & Toppenish Wastewater Treatment Plant & 1.35 & 8 & 4,800 \\
\hline Chelan (NC) & Chelan Treatment Plant & 1.2 & 8 & 4,800 \\
\hline Island (NW) & Oak Harbor, Clty of & 0.85 & 8 & 4,800 \\
\hline Columbia (SE) & Dayton, City of & 0.6 & 8 & 4,800 \\
\hline Pierce (PS) & Rainier State School & NA & 8 & 4,800 \\
\hline Whitman (SE) & Pullman Wastewater Treatment Plant & 4.3 & 8 & 4,800 \\
\hline Benton (SE) & Richland Wastewater Treatment Plant"* & 8.9 & 8 & 4,800 \\
\hline Pierce (PS) & Tacoma STP-3, City of & 10 & 5 & 3,000 \\
\hline Clallam $(W)$ & Port Angeles, City of & 3.2 & 5 & 3,000 \\
\hline King (PS) & Richmond Beach Treatment Plant & 3.2 & 5 & 3,000 \\
\hline King (PS) & Carkeek Park Treatment Plant & 3.5 & 4 & 2,400 \\
\hline Skagit (NW) & Mount Vemon, City of & 4.5 & 4 & 2,400 \\
\hline \multirow[t]{2}{*}{ Grays Harbor (W) } & McCleary Sewage Treatment Plant & 0.75 & $<1$ & 276 \\
\hline & Total & 477 & 1.349 & 810,276 \\
\hline
\end{tabular}

• Supplies Washington Natural Gas Pipeline "* Electrical Generation Capacity 
Table $11-2$

\section{Municipal Solid Waste Combustion Facilities}

Sorted by Annual Consumption

County (Region) Facility Name

Annual MSW Consumption

(Tons/Year)

Gross Energy Equivalent"

(MBturyr)

Spokane (NE) **

Pierce (PS)

Skagit (NW)

Tacoma Steam Plant \#2

Skagit Co. Resource Recycling Facil

Thermal Reduction Company

Total

292,000

98,000

50,000

36,500

476,500
$3,796,000$

$1,274,000$

$(50,000$

$4 \% 4,500$

$2,398,500$

* Energy Equivalent, 13 MBtu/Ton Municipal Solid Waste

* Under construction, operational 1992

Source: Washington State Energy Office 
Table II-3

Wood Combustion Facilities - Forest Products Industries

Sorted by Annual Wood Consumption

\begin{tabular}{|c|c|c|c|c|c|}
\hline County (Region) & Facility Name & $\begin{array}{l}\text { Biomass } \\
\text { Type }\end{array}$ & Source & $\begin{array}{r}\text { Annual Wood } \\
\text { Consumption } \\
\text { (BDT/Yr) }\end{array}$ & $\begin{array}{l}\text { Gross Energy } \\
\text { Equivalent } \\
\text { (MBtu/r) }\end{array}$ \\
\hline Cowlitz (SW) & Weyerhaeuser Company & Hog Fuel & In-house \& Purch. & 500,000 & $8,500,000$ \\
\hline $\begin{array}{l}\text { Cowlitz (SW) } \\
\text { Grays Harbor (W) }\end{array}$ & $\begin{array}{l}\text { Longview Fibre Company } \\
\text { ITT Rayonier, Inc. - Grays Harbor Di }\end{array}$ & $\begin{array}{l}\text { Hog Fuel } \\
\text { Hog Fuel }\end{array}$ & $\begin{array}{l}\text { Purchased } \\
\text { Purchased }\end{array}$ & $\begin{array}{l}232,812 \\
220,460\end{array}$ & $\begin{array}{l}3,957,804 \\
3,747,820\end{array}$ \\
\hline $\begin{array}{l}\text { Okanogan (NC) } \\
\text { Pierce (PS) }\end{array}$ & $\begin{array}{l}\text { Omak Wood Products, Inc. } \\
\text { Simpson Tacoma Kraft Company }\end{array}$ & $\begin{array}{l}\text { Hog Fuel } \\
\text { Hog Fuel }\end{array}$ & $\begin{array}{l}\text { In-house } \\
\text { Purchased }\end{array}$ & $\begin{array}{l}192,720 \\
174,000\end{array}$ & $\begin{array}{l}3,276,240 \\
2,958,000\end{array}$ \\
\hline $\begin{array}{l}\text { Whatcom (NW) } \\
\text { Snohomish (PS) }\end{array}$ & $\begin{array}{l}\text { Georgia-Pacific Corporation } \\
\text { Scott Paper }\end{array}$ & $\begin{array}{l}\text { Hog Fuel } \\
\text { Hog Fuel }\end{array}$ & $\begin{array}{l}\text { In-house \& Purch. } \\
\text { In-house \& Purch. }\end{array}$ & $\begin{array}{l}157,000 \\
154,000\end{array}$ & $\begin{array}{l}2,669,000 \\
2,618,000\end{array}$ \\
\hline $\begin{array}{l}\text { Pierce (PS) } \\
\text { Ferry (NE) }\end{array}$ & $\begin{array}{l}\text { Boise Cascade Corporation } \\
\text { Boise Cascade Corporation }\end{array}$ & $\begin{array}{l}\text { Hog Fuel } \\
\text { Hog Fuel }\end{array}$ & $\begin{array}{l}\text { Purchased } \\
\text { In-house }\end{array}$ & $\begin{array}{l}153,973 \\
138,975\end{array}$ & $\begin{array}{l}2,617,541 \\
2,362,575\end{array}$ \\
\hline $\begin{array}{l}\text { Yakima (SC) } \\
\text { Clallam (W) }\end{array}$ & $\begin{array}{l}\text { Boise Cascade Corporation } \\
\text { IT Rayonier, Inc. }\end{array}$ & $\begin{array}{l}\text { Hog Fuel } \\
\text { Hog Fuel }\end{array}$ & $\begin{array}{l}\text { In-house } \\
\text { In-house \& Purch. }\end{array}$ & $\begin{array}{r}118,000 \\
92,593\end{array}$ & $\begin{array}{l}2,006,000 \\
1,574,081\end{array}$ \\
\hline $\begin{array}{l}\text { Walla Walla (SE) } \\
\text { Mason }(W)\end{array}$ & $\begin{array}{l}\text { Boise Cascade Corporation } \\
\text { Simpson Timber Company }\end{array}$ & $\begin{array}{l}\text { Hog Fuel } \\
\text { Hog Fuel }\end{array}$ & $\begin{array}{l}\text { Purchased } \\
\text { In-house }\end{array}$ & $\begin{array}{l}88,176 \\
83,772\end{array}$ & $\begin{array}{l}1,498,992 \\
1,424,124\end{array}$ \\
\hline $\begin{array}{l}\text { Grays Harbor }(W) \\
\text { King (PS) }\end{array}$ & $\begin{array}{l}\text { Weyerhaeuser Company } \\
\text { Weyerhaeuser Company }\end{array}$ & $\begin{array}{l}\text { Hog Fuel } \\
\text { Hog Fuel }\end{array}$ & $\begin{array}{l}\text { In-house \& Purch. } \\
\text { In-house }\end{array}$ & $\begin{array}{l}80,000 \\
78,939\end{array}$ & $\begin{array}{l}1,360,000 \\
1,341,963\end{array}$ \\
\hline $\begin{array}{l}\text { Jefferson }(W) \\
\text { Clallam }(W)\end{array}$ & $\begin{array}{l}\text { Port Townsend Paper } \\
\text { Daishowa America }\end{array}$ & $\begin{array}{l}\text { Hog Fuel } \\
\text { Hog Fuel }\end{array}$ & $\begin{array}{l}\text { Purchased } \\
\text { Purchased }\end{array}$ & $\begin{array}{l}77,713 \\
63,600\end{array}$ & $\begin{array}{l}1,321,121 \\
1,081,200\end{array}$ \\
\hline $\begin{array}{l}\text { Clark (SW) } \\
\text { Clark (SW) }\end{array}$ & $\begin{array}{l}\text { James River } \\
\text { Fort Vancouver Plywood }\end{array}$ & $\begin{array}{l}\text { Hog Fuel } \\
\text { Hog Fuel }\end{array}$ & $\begin{array}{l}\text { Purchased } \\
\text { In-house }\end{array}$ & $\begin{array}{l}51,552 \\
38,500\end{array}$ & $\begin{array}{l}876,384 \\
654,500\end{array}$ \\
\hline $\begin{array}{l}\text { Snohomish (PS) } \\
\text { Clallam (W) }\end{array}$ & $\begin{array}{l}\text { Summit Timber Power House } \\
\text { K-Pty, Inc. }\end{array}$ & $\begin{array}{l}\text { Hog Fuel } \\
\text { Mil Residue }\end{array}$ & $\begin{array}{l}\text { In-house } \\
\text { Inthouse }\end{array}$ & $\begin{array}{l}33,653 \\
24,000\end{array}$ & $\begin{array}{l}572,101 \\
408,000\end{array}$ \\
\hline $\begin{array}{l}\text { Pacific }(W) \\
\text { Grays Harbor }(W)\end{array}$ & $\begin{array}{l}\text { Weyerhaeuser Company } \\
\text { Simpson Door Company }\end{array}$ & $\begin{array}{l}\text { Hog Fuel } \\
\text { Hog Fuel }\end{array}$ & $\begin{array}{l}\text { In-house } \\
\text { In-house }\end{array}$ & $\begin{array}{l}23,091 \\
22,440\end{array}$ & $\begin{array}{l}392,547 \\
381,480\end{array}$ \\
\hline $\begin{array}{l}\text { Okanogan (NC) } \\
\text { Whatcom (NW) } \\
\text { Spokane (NE) }\end{array}$ & $\begin{array}{l}\text { Colville Indian Precision Pine Co. } \\
\text { Mt. Baker Plywood } \\
\text { W.I. Forest Products }\end{array}$ & $\begin{array}{l}\text { Hog Fuel } \\
\text { Hog Fuel } \\
\text { Hog Fuel }\end{array}$ & $\begin{array}{l}\text { In-house } \\
\text { In-house } \\
\text { In-house }\end{array}$ & $\begin{array}{l}21,420 \\
19,332 \\
19,332\end{array}$ & $\begin{array}{l}364,140 \\
328,644 \\
328,644\end{array}$ \\
\hline $\begin{array}{l}\text { Ferry (NE) } \\
\text { Stevens (NE) }\end{array}$ & $\begin{array}{l}\text { San Poil Lumber Company } \\
\text { Vaagen Brothers Lumber, Inc. }\end{array}$ & $\begin{array}{l}\text { Hog Fuel } \\
\text { Hog Fuel }\end{array}$ & $\begin{array}{l}\text { In-house } \\
\text { In-house }\end{array}$ & $\begin{array}{l}18,795 \\
17,885\end{array}$ & $\begin{array}{l}319,515 \\
304,045\end{array}$ \\
\hline $\begin{array}{l}\text { Lewis (SW) } \\
\text { Pierce (PS) }\end{array}$ & $\begin{array}{l}\text { Comlitz Stud Company } \\
\text { Buffelen Woodworking }\end{array}$ & $\begin{array}{l}\text { Hog Fuel } \\
\text { Mill Residue }\end{array}$ & $\begin{array}{l}\text { In-house } \\
\text { In-house }\end{array}$ & $\begin{array}{l}16,647 \\
15,573\end{array}$ & $\begin{array}{l}282,999 \\
264,741\end{array}$ \\
\hline $\begin{array}{l}\text { Thurston (PS) } \\
\text { Clark (SW) }\end{array}$ & $\begin{array}{l}\text { Hardel Mutual Plywood } \\
\text { Pacific Wood Treating }\end{array}$ & $\begin{array}{l}\text { Mill Residue } \\
\text { Hog Fuel }\end{array}$ & $\begin{array}{l}\text { In-house } \\
\text { In-house \& Purch. }\end{array}$ & $\begin{array}{l}15,573 \\
13,750\end{array}$ & $\begin{array}{l}264,741 \\
233,750\end{array}$ \\
\hline $\begin{array}{l}\text { Lewis (SW) } \\
\text { Skamania (SW) }\end{array}$ & $\begin{array}{l}\text { Champion Intemational } \\
\text { Stevenson Co-Ply Inc. }\end{array}$ & $\begin{array}{l}\text { Hog Fuel } \\
\text { Hog Fuel }\end{array}$ & $\begin{array}{l}\text { In-house } \\
\text { in-house }\end{array}$ & $\begin{array}{l}12,579 \\
12,485\end{array}$ & $\begin{array}{l}213,843 \\
212,245\end{array}$ \\
\hline $\begin{array}{l}\text { Skagit (NW) } \\
\text { Pierce (PS) }\end{array}$ & $\begin{array}{l}\text { Goodyear Nelson Hardwood Co. } \\
\text { Puget Sound Plywood }\end{array}$ & $\begin{array}{l}\text { Hog Fuel } \\
\text { Mill Residue }\end{array}$ & $\begin{array}{l}\text { In-house } \\
\text { In-house \& Purch. }\end{array}$ & $\begin{array}{l}12,351 \\
11.900\end{array}$ & $\begin{array}{l}209,967 \\
202,300\end{array}$ \\
\hline Snohomish (PS) & Northwest Hardwoods & Mill Residue & In-house & $11,2 \pi 7$ & 191,709 \\
\hline Clallam (W) & Allen Logging Company & Mill Residue & In-house & 10,752 & 182,784 \\
\hline $\begin{array}{l}\text { Lewis (SW) } \\
\text { Yakima (SC) }\end{array}$ & $\begin{array}{l}\text { Cowlitz Stud Company } \\
\text { Jeld-Wen Fiber of WA }\end{array}$ & $\begin{array}{l}\text { Hog Fuel } \\
\text { Hog Fuel }\end{array}$ & $\begin{array}{l}\text { In-house } \\
\text { In-house }\end{array}$ & $\begin{array}{r}10,740 \\
8,750\end{array}$ & $\begin{array}{l}182,580 \\
148,750\end{array}$ \\
\hline Walla Walla (SE) & Louisiana Pactic Company & Hog Fuel & In-house & 8,400 & 142,800 \\
\hline Asotin (SE) & Bennett Lumber Products & Hog Fuel & In-house & 8,030 & 136,510 \\
\hline Snohomish (PS) & E.A. Nord & Hog Fuel & In-house & 7,000 & 119,000 \\
\hline
\end{tabular}




\begin{tabular}{|c|c|c|c|c|c|}
\hline County (Region) & Facility Name & $\begin{array}{l}\text { Biomass } \\
\text { Type }\end{array}$ & Source & $\begin{array}{r}\text { Annual Wood } \\
\text { Consumption } \\
\text { (BDT/Yr) }\end{array}$ & $\begin{array}{r}\text { Gross Energy } \\
\text { Equivalent } \\
(\mathrm{MBtu} / \mathrm{Yr})\end{array}$ \\
\hline $\begin{array}{l}\text { Pend Oreille (NE) } \\
\text { Snohomish (PS) }\end{array}$ & $\begin{array}{l}\text { Vaagen Brothers Lumber, Inc. } \\
\text { West Coast Forest Products, Inc. }\end{array}$ & $\begin{array}{l}\text { Hog Fuel } \\
\text { Mill Residue }\end{array}$ & $\begin{array}{l}\text { In-house } \\
\text { In-house }\end{array}$ & $\begin{array}{l}6,643 \\
4,720\end{array}$ & $\begin{array}{r}112,931 \\
80,240\end{array}$ \\
\hline $\begin{array}{l}\text { Walla Walla (SE) } \\
\text { Yakima (SC) }\end{array}$ & $\begin{array}{l}\text { Strauser Manufacturing } \\
\text { Layman's Lumber Company }\end{array}$ & $\begin{array}{l}\text { Hog Fuel } \\
\text { Hog Fuel }\end{array}$ & $\begin{array}{l}\text { In-house } \\
\text { In-house }\end{array}$ & $\begin{array}{l}3,000 \\
2,100\end{array}$ & $\begin{array}{l}51,000 \\
35,700\end{array}$ \\
\hline $\begin{array}{l}\text { Lewis (SW) } \\
\text { Lewis (SW) }\end{array}$ & $\begin{array}{l}\text { DeGoed Bulb Fams } \\
\text { Kinnear of Washington }\end{array}$ & $\begin{array}{l}\text { Mill Residue } \\
\text { Hog Fuel }\end{array}$ & $\begin{array}{l}\text { Purchased } \\
\text { in-house }\end{array}$ & $\begin{array}{r}1,625 \\
770\end{array}$ & $\begin{array}{l}27,625 \\
13,090\end{array}$ \\
\hline $\begin{array}{l}\text { Whatcom (NW) } \\
\text { Pierce (PS) }\end{array}$ & $\begin{array}{l}\text { Oeser Company, The } \\
\text { Fred Tebb \& Sons, Inc. }\end{array}$ & $\begin{array}{l}\text { Hog Fuel } \\
\text { Hog Fuel }\end{array}$ & $\begin{array}{l}\text { In-house } \\
\text { in-house }\end{array}$ & $\begin{array}{l}280 \\
153\end{array}$ & $\begin{array}{l}4,760 \\
2,601\end{array}$ \\
\hline Pierce (PS) & Coastcraft, Inc. & Mill Residue & In-house & NA & NA \\
\hline Stevens (NE) & PlumCreek Manufacturing, Inc. & Hog Fuel & Inthouse & NA & NA \\
\hline \multirow[t]{2}{*}{ Snohomish (PS) } & Smith Street Mill, Inc. & Hog Fuel & In-house & NA & NA \\
\hline & Total & & & $3,091,831$ & $52,561,127$ \\
\hline
\end{tabular}

Table II-4

\section{Wood Combustion Facilities - State}

Sorted by Annual Wood Consumption

Annual Wood

Biomass

Type Used Source
Consumption

(BDTMR)
Gross Energy

Equivalent

(MBtu)

County (Region) Facility Name

$\begin{array}{ll}\text { Pierce (PS) } & \text { Westem State Hospital } \\ \text { Walla Walla (SE) } & \text { Washington State Penitentiary }\end{array}$

Lewis (SW)

Pierce (PS)

Maple Lane School
Washington Soldiers Home \& Colon Wood pellet Purchased

Total
Wood pellet Purchased

Wood pellet Purchased

Wood pellet Purchased

Wood pellet Purchased
12,000

3,000

2,043

1,930

18,973
204,000

51,000

34,731

32,810

322,541

Table II-5

\section{Wood Combustion Facilities - Utility Companies}

\begin{tabular}{llllrl} 
County (Region) & Facility Name & $\begin{array}{l}\text { Biomass } \\
\text { Type Used Source }\end{array}$ & $\begin{array}{r}\text { Biomass Wood } \\
\text { Consumption } \\
\text { (BDTMR) }\end{array}$ & $\begin{array}{r}\text { Gross Energy } \\
\text { Equivalent } \\
\text { (MBtu/Yr) }\end{array}$ \\
\hline Ferry (NE) $\quad$ Washington Water Power Co. & Hog Fuel $\quad$ Purchased & 350,000 & $5,950,000$
\end{tabular}


Table II-6

Solid Fuel Processing Facilities

Sorted by Plant Capacty

Average Gross

Plant Capacity Plant Output Energy Equivalent

County (Region) Facility Name

(Tons $/ \mathrm{Yr}$ )

(Tons/Yr)

(M Btu)

\begin{tabular}{llrrr}
\hline & & & & \\
Pierce (PS) & Manke Lumber Company & 74,880 & 74,880 & $1,272,960$ \\
Spokane (NE) & Spokane Pres-To-Logs & 69,000 & 52,000 & 884,000 \\
Grays Harbor (W) & Wood Pellet Company & 36,500 & 3,650 & 62,050 \\
Okanogan (NC) & CD Pellet Company & 6,000 & 6,000 & 102,000 \\
& & & & \\
& Total & 186,380 & 136,530 & $2,321,010$
\end{tabular}


Part III

Potential Demand 


\section{Part III}

\section{Potential Demand}

Major savings can be realized by a number of energy-intensive facilities in both the public and private sectors of Washington state. There are approximately 128 state agencies, 39 colleges and universities, $500 \mathrm{~K}-12$ public and private schools, 111 hospitals, and hundreds of industrial facilities statewide. Although all these facilities may not find biomass fuels an economically feasible alternative to electricity, natural gas, coal, or oil, larger facilities may find it advantageous to consider their use. A rough estimate of potential markets for biomass fuels is derived from the information provided in this section.

The commercial sector (which includes state buildings, hospitals, schools) and industrial sector require vast amounts of hot water, heat, and air-conditioning, as well as electricity. Some facilities require round-the-clock services. If their energy needs can be met more efficiently, appreciable reductions in operating costs could result in saving taxpayers and businesses thousands of dollars each year. Large facilities $m$ y benefit from installing cogeneration capability using biomass to supplement their energy requirements. These facilities would have the added advantage of selling electricity to utilities. Medium-sized facilities may find switching to natural gas to be the answer. Small facilities may want to increase the energy efficiency of the building structure. Facility managers must select and develop the best energy options to meet their needs.

Table III-1, page III-3 shows the total er ergy consumption in Washington state by sector.

A list of facilities and other pertinent data on a county-by-county basis can be obtained from the Washington State Energy Office (WSEO).

Table III-2, page III-4 shows the average square footage of state buildings by county. Data were obtained from WSEO's Energy Partnerships program. The average square feet of state buildings by county may not include all state facilities in the county; rather, it is an average of the major ones.

Table III-3, page III-5 shows the average square footage of school buildings by county. Data presented were obtained from Superintendent of Public Instruction. Square footage by county was for all schools-large, medium, and small-for that particular county. 


\section{Hospitals}

\section{Industrial Users}


Table III-1

Washinton State Total Energy Consumption by Sector* Billion Btu

\begin{tabular}{|c|c|c|c|c|c|c|}
\hline Year & Residential & Commercial & Industrial & Transportation & Other** & Total"*** \\
\hline 1960 & 83,290 & 36,696 & 195,477 & 170,076 & 4,913 & 490,451 \\
\hline $\begin{array}{l}1961 \\
1962\end{array}$ & $\begin{array}{l}84,793 \\
86,319\end{array}$ & $\begin{array}{l}38,188 \\
38,834\end{array}$ & $\begin{array}{l}189,537 \\
203,666\end{array}$ & $\begin{array}{l}174,326 \\
181,503\end{array}$ & $\begin{array}{l}5,415 \\
5,589\end{array}$ & $\begin{array}{l}492,258 \\
515,911\end{array}$ \\
\hline $\begin{array}{l}1963 \\
1964\end{array}$ & $\begin{array}{r}91,112 \\
101,202\end{array}$ & $\begin{array}{l}39,397 \\
45,989\end{array}$ & $\begin{array}{l}211,443 \\
234,303\end{array}$ & $\begin{array}{l}183,881 \\
189,346\end{array}$ & $\begin{array}{l}6,224 \\
6,735\end{array}$ & $\begin{array}{l}532,056 \\
577,576\end{array}$ \\
\hline $\begin{array}{l}1965 \\
1966\end{array}$ & $\begin{array}{r}99,374 \\
106,326\end{array}$ & $\begin{array}{l}45,149 \\
48,947\end{array}$ & $\begin{array}{l}242,963 \\
250,981\end{array}$ & $\begin{array}{l}196,940 \\
214,031\end{array}$ & $\begin{array}{l}7,097 \\
8,100\end{array}$ & $\begin{array}{l}591,523 \\
628,384\end{array}$ \\
\hline $\begin{array}{l}1967 \\
1968\end{array}$ & $\begin{array}{l}107,615 \\
120,053\end{array}$ & $\begin{array}{l}50,332 \\
54,439\end{array}$ & $\begin{array}{l}258,039 \\
271,388\end{array}$ & $\begin{array}{l}235,981 \\
268,404\end{array}$ & $\begin{array}{l}8,424 \\
8,765\end{array}$ & $\begin{array}{l}660,390 \\
723,049\end{array}$ \\
\hline $\begin{array}{l}1969 \\
1970\end{array}$ & $\begin{array}{l}131,261 \\
136,441\end{array}$ & $\begin{array}{l}58,051 \\
61,331\end{array}$ & $\begin{array}{l}282,854 \\
281,154\end{array}$ & $\begin{array}{l}275,837 \\
276,092\end{array}$ & $\begin{array}{l}9,032 \\
9,543\end{array}$ & $\begin{array}{l}757,034 \\
764,562\end{array}$ \\
\hline $\begin{array}{l}1971 \\
1972\end{array}$ & $\begin{array}{l}143,407 \\
148,724\end{array}$ & $\begin{array}{l}65,711 \\
70,066\end{array}$ & $\begin{array}{l}276,372 \\
306,563\end{array}$ & $\begin{array}{l}283,242 \\
286,951\end{array}$ & $\begin{array}{l}10,284 \\
11,075\end{array}$ & $\begin{array}{l}779,017 \\
823,381\end{array}$ \\
\hline $\begin{array}{l}1973 \\
1974\end{array}$ & $\begin{array}{l}144,210 \\
136,866\end{array}$ & $\begin{array}{l}79,613 \\
77,628\end{array}$ & $\begin{array}{l}320,587 \\
305,064\end{array}$ & $\begin{array}{l}313,712 \\
315,595\end{array}$ & $\begin{array}{l}13,815 \\
13,201\end{array}$ & $\begin{array}{l}871,944 \\
848,355\end{array}$ \\
\hline 1975 & 139,898 & 77,423 & 269,524 & 338,250 & 13,993 & 839,099 \\
\hline 1976 & 143,385 & 79,571 & 262,705 & 359,640 & 15,112 & 860,412 \\
\hline $\begin{array}{l}1977 \\
1978\end{array}$ & $\begin{array}{l}148,593 \\
150,566\end{array}$ & $\begin{array}{l}81,891 \\
80,682\end{array}$ & $\begin{array}{l}268,486 \\
270,573\end{array}$ & $\begin{array}{l}369,752 \\
397,293\end{array}$ & $\begin{array}{l}16,115 \\
15,016\end{array}$ & $\begin{array}{l}884,837 \\
914,130\end{array}$ \\
\hline 1979 & 159,223 & 89,246 & 272,166 & 426,916 & 17,927 & 965,477 \\
\hline 1980 & 148,001 & 87,755 & 262,909 & 409,940 & 15,040 & 923,656 \\
\hline $\begin{array}{l}1981 \\
1982\end{array}$ & $\begin{array}{l}141,918 \\
146,844\end{array}$ & $\begin{array}{l}86,215 \\
99,284\end{array}$ & $\begin{array}{l}277,369 \\
244,151\end{array}$ & $\begin{array}{l}400,504 \\
375,950\end{array}$ & $\begin{array}{l}17,531 \\
16,555\end{array}$ & $\begin{array}{l}923,536 \\
882,784\end{array}$ \\
\hline $\begin{array}{l}1983 \\
1984\end{array}$ & $\begin{array}{l}134,416 \\
143,195\end{array}$ & $\begin{array}{r}97,336 \\
104,293\end{array}$ & $\begin{array}{l}219,042 \\
244,239\end{array}$ & $\begin{array}{l}362,326 \\
393,292\end{array}$ & $\begin{array}{l}15,791 \\
16,330\end{array}$ & $\begin{array}{l}828,912 \\
901,349\end{array}$ \\
\hline 1985 & 151,417 & 121,527 & 224,354 & 415,601 & 18,220 & 931,119 \\
\hline 1986 & 142,290 & 103,011 & 236,002 & 494,934 & 13,946 & 990,184 \\
\hline 1987 & 139,809 & 105,432 & 238,029 & 497,756 & 14,338 & 995,364 \\
\hline 1988 & 153,642 & 115,854 & 246,794 & 526,933 & 10,162 & $1,053,385$ \\
\hline
\end{tabular}

Source: U.S. Department of Energy 
Table III-2

Average Square Feet of State Buildings By County

Sorted by Number of Square Feet

\begin{tabular}{lrrrr}
\hline & $\begin{array}{r}\text { Number of } \\
\text { Facilities }\end{array}$ & $\begin{array}{r}\text { Average } \\
\text { Sq. Feet }\end{array}$ & Mninimum & Maximum \\
County (Region) & & & & \\
& & & & \\
& & 186,573 & 1,864 & $1,885,930$ \\
Thurston (PS) & 19 & 758,451 & 4,500 & $10,344,000$ \\
King (PS) & 11 & 413,333 & 14,654 & $2,017,730$ \\
Spokane (NE) & 10 & 395,187 & 7,360 & $1,208,629$ \\
Pierce (PS) & 7 & 147,113 & 13,452 & 422,800 \\
Clark (SW) & 6 & 101,320 & 6,348 & 343,508 \\
Yakima (SC) & 6 & 103,229 & 5,600 & 206,092 \\
Lewis (SW) & 6 & 270,704 & 11,652 & 801,012 \\
Snohomish (PS) & 5 & 83,082 & 6,252 & 221,550 \\
Chelan (NC) & 4 & 126,158 & 42,677 & 266,552 \\
Clallam (W) & 4 & 328,342 & 12,000 & 713,440 \\
Kitsap (PS) & 3 & 861,543 & 62,525 & $2,319,314$ \\
Whatcom (NW) & 3 & 104,015 & 34,014 & 232,241 \\
Skagit (NW) & 3 & 260,114 & 49,829 & 673,589 \\
Mason (W) & 2 & $1,203,212$ & 9,505 & $2,396,918$ \\
Kittitas (SC) & 2 & 689,534 & 315,011 & $1,064,056$ \\
Walla Walla (SE) & 2 & 196,061 & 5,832 & 386,289 \\
Grant (NC) & 2 & $4,179,397$ & 17,194 & $8,341,600$ \\
Whitman (SE) & 1 & 123,236 & 240,787 \\
Benton (SE) & 1 & 272,711 & 272,711 & 272,711 \\
Cowlitz (SW) & 1 & 302,650 & 302,650 & 302,650 \\
Franklin (SE) & 1 & 143,065 & 143,065 & 143,065 \\
Pacific (W) & 1 & $18,35 ?$ & 18,352 & 18,352 \\
Okanogan (NC) & 1 & 188,218 & 188,218 & 188,218 \\
Grays Harbor (W) & & 79,934 & 79,934 & 79,934 \\
San Juan (NW) & & & &
\end{tabular}


Table III-3

\section{Average Square Feet of School Buildings By County}

Ranked by Average Building Size (Sq.Ft.)

\begin{tabular}{|c|c|c|c|c|}
\hline Rank & County (Region) & $\begin{array}{l}\text { Average } \\
\text { Building } \\
\text { Size } \\
\text { (Sq.Ft.) }\end{array}$ & $\begin{array}{l}\text { Minimum } \\
\text { Building } \\
\text { Size } \\
\text { (Sq.Ft.) }\end{array}$ & $\begin{array}{l}\text { Maximum } \\
\text { Building } \\
\text { Size } \\
\text { (Sq.Ft.) }\end{array}$ \\
\hline 1 & Kitsap (PS) & 71,111 & 19,616 & 337,482 \\
\hline 2 & King (PS) & 66,548 & 3,600 & 323,937 \\
\hline 3 & Benton (SE) & 65,234 & 800 & 271,553 \\
\hline 4 & Snohomish (PS) & 64,707 & 3,010 & 285,674 \\
\hline 5 & Pierce (PS) & 63,398 & 1,720 & 325,706 \\
\hline 6 & Clark (SW) & 63,143 & 3,496 & 228,550 \\
\hline 7 & Spokane (NE) & 61,587 & 1,717 & 260,025 \\
\hline 8 & Island (NW) & 60,222 & 35,502 & 137,191 \\
\hline 9 & Thurston (PS) & 59,148 & 17,214 & 171,670 \\
\hline 10 & Yakima (SC) & 58,876 & 15,395 & 248,428 \\
\hline 11 & Cowlitz (SW) & 58,638 & 4,960 & 181,653 \\
\hline 12 & Whatcom (NW) & 57,583 & 8,170 & 188,466 \\
\hline 13 & Franklin (SE) & 56,882 & 8,860 & 242,092 \\
\hline 14 & Walla Walla (SE) & 55,885 & 11,576 & 171,146 \\
\hline 15 & Chelan (NC) & 51,400 & 770 & 259,409 \\
\hline 16 & Clallam (W) & 50,746 & 8,066 & 200,904 \\
\hline 17 & Mason (W) & 50,399 & 12,700 & 177,624 \\
\hline 18 & Gartield (SE) & 49,206 & 36,848 & 61,563 \\
\hline 19 & Grays Harbor (W) & 48,943 & 3,635 & 265,857 \\
\hline 20 & Skagit (NW) & 47,437 & 1.716 & 186,145 \\
\hline 21 & Skamania (SW) & 45,804 & 8,815 & 122,127 \\
\hline 22 & Lewis (SW) & 45,148 & 5,425 & 142,794 \\
\hline 23 & Douglas (NC) & 43,902 & 3,440 & 146,847 \\
\hline 24 & Kittitas (SC) & 42,596 & 4,725 & 147,466 \\
\hline 25 & Okanogan (NC) & 42,237 & 4,955 & 139,063 \\
\hline 26 & Jetferson $(W)$ & 41,936 & 9,664 & 99,913 \\
\hline 27 & Whitman (SE) & 41,293 & 1,000 & 154,085 \\
\hline 28 & Grant (NC) & 41,290 & 7,185 & 124,638 \\
\hline 29 & Asotin (SE) & 41,087 & 1,800 & 133,984 \\
\hline 30 & Adams (SE) & 40,553 & 5,347 & 109,767 \\
\hline 31 & Lincoln (NE) & 37,334 & 9,695 & 87.641 \\
\hline 32 & Pend Orelle (NE) & 37,086 & 10,894 & 77,581 \\
\hline 33 & Stevens (NE) & 36,961 & 2,324 & 62,625 \\
\hline 34 & Klickitat (SC) & 36,528 & 6,523 & 73,920 \\
\hline 35 & Wahkiakum (SW) & 34,779 & 30.239 & 39,319 \\
\hline 36 & Pacific (W) & 33,595 & 14,260 & 89,249 \\
\hline 37 & Ferry (NE) & 28,054 & 897 & 58,116 \\
\hline 38 & Columbia (SE) & 27,181 & 12.018 & 49,247 \\
\hline 39 & San Juan (NW) & 25.453 & 900 & 71,272 \\
\hline
\end{tabular}

Source: Office of State Superintendent of Public Instruction 
Table III-4

\section{Washington State Hospitals}

Sorted by Total Number of Beds

County (Region)
Hospital Name

Total Beds
King (PS)

Spokane (NE)

King (PS)

Pierce (PS)

King (PS)

Spokane (NE)

King (PS)

Cowlitz (SW)

King (PS)

Thurston (PS)

Pierce (PS)

King (PS)

King (PS)

Kitsap (PS)

King (PS)

Spokane (NE)

Grays Harbor (W)

Snohomish (PS)

Clark (SW)

Pierce (PS)

Yakima (SC)

King (PS)

Snohomish (PS)

Snohomish (PS)

Yakima (SC)

King (PS)

King (PS)

Clark (SW)

Lewis (SW)

King (PS)

King (PS)

Chelan (NC)

King (PS)

Pierce (PS)

Pierce (PS)

Benton (SE)

King (PS)

Walla Walla (SE)

Spokane (NE)

King (PS)

Skagit (NW)

Whatcom (NW)

King (PS)
Swedish Hospital Medical Center $\quad 697$

Sacred Heart Medical Center $\quad 615$

University Hospital $\quad 450$

Tacoma General Hospital $\quad 415$

Harborview Medical Center $\quad 413$

Deaconess Medical Center $\quad 388$

Providence Medical Center $\quad 376$

St.John's Medical Center $\quad 346$

Group Health Cooperative Central Hospital $\quad 346$

St.Peter Hospital $\quad 340$

St.Joseph Hospital \& Health Care Center $\quad 340$

Virginia Mason Hospital $\quad 336$

Valley Medical Center 303

Harrison Memorial Hospital 297

Northwest Hospital $\quad 281$

Holy Family Hospital 272

Grays Harbor Community Hospital 259

General Hospital Medical Center 257

St. Joseph Community Hospital 243

Good Samaritan Hospital and Rehabilitation Center 225

Yakima Valley Memorial Hospital 225

Overlake Hospital Medical Center 218

Providence Hospital 218

Stevens Memorial Hospital 217

St. Elizabeth Medical Center $\quad 214$

Children's Hospital and Medical Center 208

West Seattle Community Hospital 198

Vancouver Mernorial Hospital 192

Providence Hospital-Centralia 191

St. Frances Xavier Cabrini Hospital 189

Group Health Eastside Hospital $\quad 179$

Central Washington Hospital $\quad 176$

Ballard Community Hospital 163

Puget Sound Hospital 160

Humana Hospital Tacoma $\quad 155$

Kadlec Hospital 153

Auburn General Hospital $\quad 149$

St. Mary Meciical Center $\quad 146$

St. Luke's Memorial Hospital 146

Rlverton General Hospital $\quad 142$

Skagit Valley Hospital \& Health Center 137

St. Joseph Hospital 136

Highline Community Hospital $\quad 135$ 
Table I!I-4

\section{Washington State Hospitals (Continued)}

Sorted by Total Number of Beds

County (Region)

Hospltal Name

Total Beds

King (PS)

King (PS)

Clallam (W)

Spokane (NE)

Franklin (SE)

Whatcom (NW)

Thurston (PS)

King (PS)

Pierce (PS)

Okanogan (NC)

Skagit (NW)

Lincoln (NE)

King (PS)

King (PS)

Benton (SE)

Walla Walla (SE)

Snohomish (PS)

Benton (SE)

Mason (W)

Yakima (SC)

King (PS)

Asotin (SE)

Grant (NC)

Pierce (PS)

Stevens (NE)

Gartield (SE)

Island (NW)

Grant (NC)

Kittitas (SC)

Adams (SE)

Snohomish (PS)

Whitman (SE)

Grant (NC)

Okanogan (NC)

Lincoln (NE)

Okanogan (NC)

Skagit (NW)

Jefferson (W)

Whitman (SE)

Thurston (PS)

Yakima (SC)

King (PS)

Clallam (W)

Pacific (W)

Spokane (NE)
Evergreen Hospital Medical Center

134

Fairfax Hospital 133

Olympic Memorial Hospltal 126

Valley Hospital and Medical Center 123

Our Lady of Lourdes Hospital 119

St. Lukes's General Hospital $\quad 117$

Black Hills Community Hospital $\quad 110$

St. Frances Community Hospital $\quad 110$

Lakewood Hospital

North Valley Hospital

105

Lincoln Hospital

Careunit Hospital of Kirkland 83

Fifth Avenue Medical Center $\quad 80$

Prosser Memorial Hospital $\quad 75$

Walla Walla General Hospital 72

Valley General Hospital 72

Kennewick General Hospital $\quad 71$

Mason General Hospital $\quad 68$

Providence Central Memorial Hospital 63

Schick-Shadel Hospital 63

Tri-State Memorial Hospital, Inc. 62

Columbia Basin Hospital $\quad 58$

Mary Bridge Children's Health Center 56

Mount Carmel Hospital 55

Gartield County Memorial Hospital $\quad 54$

Whidbey General Hospital $\quad 51$

Samaritan Hospital $\quad \mathbf{5 0}$

Kittitas Valley Community Hospital $\quad 50$

Othello Community Hospital $\quad 49$

Cascade Valley Hospital 48

Whitman Community Hospital 48

Coulee Community Hospital 48

Mid-Valley Hospltal 44

Odessa Memorial Hospital $\quad 44$

Okanogan-Douglas County Hospital 43

Island Hospital 43

Jefferson General Hospital 42

Pullman Memorial Hospital 42

Cascade Oaks $\quad 40$

Sunnyside Community Hospital 38

Community Memorial Hospital 38

Forks Community Hospital $\quad 37$

Willapa Harbor Hospital 36

Mountainview Hospital 34 
Table III-4

Washington State Hospitals (Continued)

Sorted by Total Number of Beds

County (Region) Hospital Name

Total Beds

Chelan (NC)

Chelan (NC)

Benton (SE)

Klickitat (SC)

Klickitat (SC)

Yakima (SC)

Spokane (NE)

King (PS)

Columbia (SE)

King (PS)

Pierce (PS)

Spokane (NE)

Stevens (NE)

Ferry (NE)

Pacific (W)

Pend Oreille (NE)

Grays Harbor (W)

Lewis (SW)

Adams (SE)

Pend Oreille (NE)

Grant (NC)

Cowlitz (SW)

Lake Chelan Community Hospital

Cascade General Hospital

33

Carondelet Psychiatric Care Center

32

Skyline Hospltal

Klickitat Valley Hospital

31

New Valley Osteopathic Hospital 30

Shriners Hospitals for Crippled Children $\quad 30$

Snoqualmie Valley Hospital 28

Dayton General Hospital 28

Medical Dental Building Hospital 26

Lakewood Hospital-New Beginnings 2S

Deer Park Health Center and Hospital 26

St. Joseph's Hospltal of Chewelah 25

Ferry County Memorial Hospital 25

Ocean Beach Hospital 25

Newport Community Hospital 24

Mark Reed Hospital 24

Morton General Hospital $\quad 20$

Ritzville Memorial Hospital 20

Mount Linton Hospital $\quad 19$

Quincy Valley Hospital 16

Hospice Care Center Hospital 4

Note: Their are many variables associated with the amount of natural gas, oil and propane consumed per hospital bed. Not all hospitals use these energy resources in addition to electricity. Where electricity is unusually inexpensive it is the only source of energy. Two examples of the therms per hospital bed are given to demonstrate how energy consumption can vary per hospltal bed. Evergreen Hospltal Medical Center in King Co. used 399,859.5 therms from 1987-1988 they have 134 beds, 399,859 therms / 134 beds $=2,984$ therms per hospital bed; St. Mary's Hospital in Walla Walla Co. used 611,235 therms from 1987.1988 they have 146 beds, 611,235 therms / 146 beds $=4,186.54$ therms per hospital bed. 
Table III-5

Average Number of Hospital Beds By Region

Sorted by Number of Hospitals

\begin{tabular}{|c|c|c|c|c|}
\hline \multirow[b]{2}{*}{ Region } & \multirow[b]{2}{*}{ Number of Hospitals } & \multirow[b]{2}{*}{ Average Number of Beds } & \multicolumn{2}{|c|}{-Range } \\
\hline & & & Minimum & Maximum \\
\hline Puget Sound & 43 & 199 & 26 & 697 \\
\hline Southeast & 20 & 98 & 4 & 346 \\
\hline Northeast & 15 & 128 & 19 & 615 \\
\hline North Central & 10 & 60 & 16 & 176 \\
\hline South Central & 8 & 85 & 30 & 225 \\
\hline West & 8 & 77 & 24 & 259 \\
\hline Northwest & 6 & 97 & 43 & 137 \\
\hline
\end{tabular}


Table III-6

Average Number of Hospital Beds per County

Ranked by Number of Hospitals

\begin{tabular}{|c|c|c|c|c|c|}
\hline Rank & County & $\begin{array}{r}\text { \# of } \\
\text { Hospitals }\end{array}$ & $\begin{array}{l}\text { Average } \\
\text { \# of Beds }\end{array}$ & $\begin{array}{l}\text { Minimum } \\
\# \text { of Beds }\end{array}$ & $\begin{array}{l}\text { Maximum } \\
\text { \# of Beds }\end{array}$ \\
\hline 1 & King (PS) & 27 & 211 & 26 & 697 \\
\hline 2 & Spokane (NE) & 10 & 204 & 26 & 615 \\
\hline 3 & Pierce (PS) & 8 & 185 & 26 & 415 \\
\hline 4 & Snohomish (PS) & 5 & 162 & 48 & 257 \\
\hline 5 & Yakima (SC) & 5 & 114 & 30 & 225 \\
\hline 6 & Benton (SE) & 4 & 83 & 32 & 153 \\
\hline 7 & Grant (NC) & 4 & 43 & 16 & 58 \\
\hline 8 & Okanogan (NC) & 3 & 61 & 43 & 97 \\
\hline 9 & Skagit (NW) & 3 & 92 & 43 & 137 \\
\hline 10 & Chelan (NC) & 3 & 81 & 33 & 176 \\
\hline 11 & Thurston (PS) & 3 & 163 & 40 & 340 \\
\hline 12 & Pacific (W) & 2 & 31 & 25 & 36 \\
\hline 13 & Lincoln (NE) & 2 & 69 & 44 & 93 \\
\hline 14 & Clark (SW) & 2 & 218 & 192 & 243 \\
\hline 15 & Whitman (SE) & 2 & 45 & 42 & 48 \\
\hline 16 & Stevens (NE) & 2 & 40 & 25 & 55 \\
\hline 17 & Klickitat (SC) & 2 & 32 & 31 & 32 \\
\hline 18 & Adams (SE) & 2 & 35 & 20 & 49 \\
\hline 19 & Walla Walla (SE) & 2 & 109 & 72 & 146 \\
\hline 20 & Lewis (SW) & 2 & 106 & 20 & 191 \\
\hline 21 & Pend Oreille (NE) & 2 & 22 & 19 & 24 \\
\hline 22 & Whatcom (NW) & 2 & 127 & 117 & 136 \\
\hline 23 & Grays Harbor (W) & 2 & 142 & 24 & 259 \\
\hline 24 & Cowlitz (SW) & 2 & 175 & 4 & 346 \\
\hline 25 & Clallam (W) & 2 & 82 & 37 & 126 \\
\hline 26 & Ferry (NE) & 1 & 25 & 25 & 25 \\
\hline 27 & Columbia (SE) & 1 & 28 & 28 & 28 \\
\hline 28 & Jefferson (W) & 1 & 42 & 42 & 42 \\
\hline 29 & Asotin (SE) & 1 & 62 & 62 & 62 \\
\hline 30 & Mason (W) & 1 & 68 & 68 & 68 \\
\hline 31 & Franklin (SE) & 1 & 119 & 119 & 119 \\
\hline 32 & Gartield (SE) & 1 & 54 & 54 & 54 \\
\hline 33 & Kitsap (PS) & 1 & 297 & 297 & 297 \\
\hline 34 & Kittitas (SC) & 1 & 50 & 50 & 50 \\
\hline 35 & Island (NW) & 1 & 51 & 51 & 51 \\
\hline
\end{tabular}


Table III-7

Fuel Consumed by Potential Industrial Biomass Users

by County

Sorted by Number of Business per County

\begin{tabular}{|c|c|c|c|c|}
\hline \multirow[b]{2}{*}{ County (Region) } & \multirow[b]{2}{*}{ \# of Businesses } & \multirow{2}{*}{$\begin{array}{l}\text { Average } \\
\text { Billion Btu }\end{array}$} & \multicolumn{2}{|c|}{ 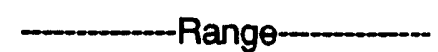 } \\
\hline & & & Minimum & Maximum \\
\hline King (PS) & 65 & 157 & 4 & 3,334 \\
\hline Pierce (PS) & 40 & 209 & 11 & 1,802 \\
\hline Yakima (SC) & 19 & 119 & 17 & 519 \\
\hline Snohomish (PS) & 17 & 639 & 4 & 6,876 \\
\hline Clark (SW) & 14 & 880 & 11 & 7,782 \\
\hline Whatcom (NW) & 13 & 894 & 20 & 3,816 \\
\hline Spokane (NE) & 13 & 162 & 19 & 1,478 \\
\hline Lewis (SW) & 10 & 45 & 15 & 148 \\
\hline Grays Harbor (W) & 9 & 247 & 23 & 996 \\
\hline Cowlitz (SW) & 9 & 1,354 & 23 & 8,190 \\
\hline Thurston (PS) & 7 & 175 & 44 & 849 \\
\hline Chelan (NC) & 6 & 298 & 56 & 946 \\
\hline Walla Walla (SE) & 6 & 836 & 26 & 4,467 \\
\hline Skagit (NW) & 4 & 1,054 & 19 & 2,143 \\
\hline Clallam (W) & 4 & 700 & 30 & 1,493 \\
\hline Grant (NC) & 4 & 274 & 39 & 532 \\
\hline Kickitat (SC) & 3 & 367 & 46 & 1,001 \\
\hline Pactic (W) & 2 & 69 & 45 & 93 \\
\hline Kitsap (PS) & 2 & 35 & 20 & 49 \\
\hline Franklin (SE) & 2 & 340 & 325 & 355 \\
\hline Jefferson (W) & 1 & 1,480 & 1,480 & 1,480 \\
\hline Pend Oreille (NE) & 1 & 1,166 & 1,166 & 1,166 \\
\hline Okanogan (NC) & 1 & 122 & 122 & 122 \\
\hline Kittitas (SC) & 1 & 23 & 23 & 23 \\
\hline Columbia (SE) & 1 & 1,037 & 1.037 & 1,037 \\
\hline Adams (SE) & 1 & 158 & 158 & 158 \\
\hline
\end{tabular}




\section{Part IV}

\section{Prices of Competing Energy Resources}




\section{Part IV}

\section{Prices Of Competing Energy Resources}

\section{Electric Companies}

\section{Natural Gas Companies}

Coal
Information contained in this section will be useful in setting biomass prices.

Until recently, electricity generated from our rivers and streams afforded Washington's industrial, commercial, residential, and governmental sectors the benefits of abundant electricity and the lowest electrical energy rates in the country. Continued growth in our state, however, has moved Washington from an era of electrical surplus to one of projected shortfall. Changes in environmental laws may also have an enormous impact on future electric utility rates. Facilities with cogeneration potential may find greater opportunities in the 1990s and beyond to profitably produce electricity for resale to electric utilities.

Tables IV-1 through IV-2 (pages IV-3 through IV-13) compare Washington state average electric utility rates per kilowatt hour for the state as a whole and by region. Table IV-3 (pages IV-14 through IV-16) shows net generation, fuel consumption, and fuel stock by company and plant.

There are two major natural gas pipelines in Washington state (see Table IV-4, page IV-17). Pipeline companies primary purchase their supplies from another state or import it from Canada. Although the natural gas utilities purchase their supplies from Northwest Pipeline Corporation, FERC Order 436, "allows suppliers and end-users to purchase gas directly from producers instead of from pipelines" (WSEO, 1988).

Table IV-5 (page IV-8) compares Washington state natural gas utility rates and to natural and other gases produced and purchased by natural gas pipeline companies.

A large percentage of Washington industrial and commercial coal is transported by rail from Utah and Wyoming. The consumption and prices of coal by sector are reported in this section. Table IV-6 (page IV-19) compares historical data from 1960 through 1988 for the residential, commercial, industrial, and electric utilities, giving consumption and prices for both Btu and short tons. 
An historical perspective of consumption and pricing of residential heating oil and residual fuels from 1960 through 1988, the last year of available data, is reported on Table IV-7, page IV-20. The information presented compares consumption and prices on a Btu basis while also comparing quantity of barrels consumed and prices per gallon.

Washington State Energy Office. Washington State Energy Use Profile 1960-1986. January 1988. 
Table IV-1

Washington State Average Electric Utilities Rates per Kilowatthour

Ranked by Unit Cost (Cents per kWh)

\begin{tabular}{|c|c|c|c|c|c|c|}
\hline \multirow{2}{*}{\multicolumn{2}{|c|}{$\begin{array}{r}\text { Agencies/Cooperatives } \\
\text { Rank Service Area (Region) }\end{array}$}} & \multirow[b]{2}{*}{ Type of Ownership } & \multirow[b]{2}{*}{ Year } & \multirow[t]{2}{*}{$\begin{array}{l}\text { Unit Cost* } \\
\text { (Cts/kwh) }\end{array}$} & \multirow{2}{*}{$\begin{array}{r}\text { Commercial } \\
(\text { Cis/kwh) } \\
9 / 90\end{array}$} & \multirow{2}{*}{$\begin{array}{r}\text { Industrial } \\
\text { (Cts/kwhi) } \\
8 / 90\end{array}$} \\
\hline & & & & & & \\
\hline & $\begin{array}{l}\text { Clearwater Power Co................................. } \\
\text { Asotin (SE) } \\
\text { Whitman (SE) }\end{array}$ & Cooperative & 1990 & 0.0631 & - & - \\
\hline & $\begin{array}{l}\text { Orcas Power and Light Co............................. } \\
\text { San Juan (NW) }\end{array}$ & Cooperative & 1988 & 0.0610 & - & - \\
\hline & $\begin{array}{l}\text { Tanner Electric Cooperative........................... } \\
\text { King (PS) }\end{array}$ & Cooperative & 1988 & 0.0546 & - & -- \\
\hline & $\begin{array}{l}\text { Mason County PUD No. 1........................... } \\
\text { Jefferson (W) } \\
\text { Mason (W) }\end{array}$ & Public Utility & 1988 & 0.0540 & -- & - \\
\hline & $\begin{array}{l}\text { Kootenai Electric Cooperative......................... } \\
\text { Spokane (NE) }\end{array}$ & Cooperative & 1990 & 0.0512 & - & -- \\
\hline & $\begin{array}{l}\text { Northem Lights, Inc.................................................. } \\
\text { Pend Oreille (NE) }\end{array}$ & Cooperative & 1990 & 0.0486 & -- & -- \\
\hline 7 & $\begin{array}{l}\text { Inland Power \& Light Co............................ } \\
\text { Garfield (SE) } \\
\text { Pend Oreille (NE) } \\
\text { Spokane (NE) } \\
\text { Whitman (SE) }\end{array}$ & Cooperative & 1989 & 0.0482 & -- & - \\
\hline & $\begin{array}{l}\text { Ferry County PUD } \\
\text { Ferry (NE) }\end{array}$ & Public Utility & 1989 & 0.0479 & -- & - \\
\hline & 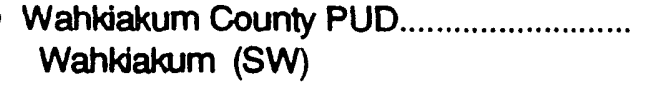 & Public Utility & 1988 & 0.0477 & - & - \\
\hline 10 & $\begin{array}{l}\text { Peninsula Light Co....................................... } \\
\text { Pierce (PS) }\end{array}$ & Cooperative & 1989 & 0.0471 & - & - \\
\hline 11 & $\begin{array}{l}\text { Okanogan County Eloctric Co-op, inc........ } \\
\text { Okanogan (NC) }\end{array}$ & Cooperative & 1988 & 0.0470 & - & - \\
\hline 12 & $\begin{array}{l}\text { Puget Sound Power and Light Co............. } \\
\text { Island (NW) } \\
\text { Jefterson (W) } \\
\text { King (PS) } \\
\text { Kitsap (PS) } \\
\text { Kittitas (SC) } \\
\text { Pierce (PS) } \\
\text { Skagit (NW) } \\
\text { Thurston (PS) } \\
\text { Whatcom (NW) }\end{array}$ & Investor Owned & 1989 & 0.0467 & 0.0529 & 0.0344 \\
\hline & 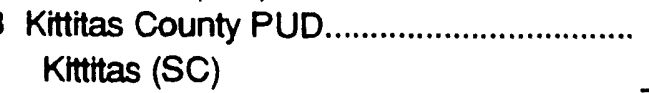 & Public Utility & 1988 & 0.0466 & - & -- \\
\hline
\end{tabular}

$-=$ Not Available 
Table IV-1

Washington State Average Electric Utilities Rates per Kilowatthour (Continued)

Agencies/Cooperatives

Rank Service Area (Region)
Unit Cost* Commercial

(Cts/kwh) (Cts/kwh)

Type of Ownership Year $9 / 90$
Industrial

(Cts/kwh)

$8 / 90$

14 OHOP Mutual Light Co

Cooperative

Pierœ (PS)

15 Mason Ccunty PUD No. 3

Public Utility

Mason (W)

16 Nespelem Valley Electric Co-op, Inc......... Cooperative Forry (NE)

Okanogan (NC)

17 Alder Mutual Light Co

Cooperative

Pierce (PS)

18 Clallam County PUD

Public Utility Jefferson (W)

19 Sumas, City of. Municipality Whatcom (NW)

20 Clallam County PUD Public Utility Clallam (W)

21 Benton Rural Electic Assoc., Inc Cooperative

1988

0.0449

1989

0.0447

1988

0.0443

1988

0.0440

1989

0.0439

1989

0.0436

1989

0.0431

1988

0.0428

Benton (SE)

Yakima (SC)

22 Pacific County PUD No. 2

Public Utility

1988

0.0424

Pacific (W)

Municipality

1988

0.0423

Pierce (PS)

24 Klickitat County PUD Public Utility Klickitat (SC)

25 Steilacoom, Town of

Other Pierce (PS)

26 Modem Ele tic Co Cooperative Spokane (NE)

27 Milton, City of.. Municipality Pierce (PS) Municipality Municipality

Public Utility

1989

1988

0.0417

0.0417

1988

0.0411

1988

0.0411

1988

0.0404

1988

0.0402

1988

0.0402

0.0342

0.0272 istand (NW) Snohomish (PS)

31 Washington Water Power Co., The Investor Owned

1988

0.0399

0.0520

0.0347

Cooperative

1389

32 Ruston Electric Utilities.

0.0398 King (PS) 


\section{Washington State Average Electric Utilities Rates per Kil owatthour (Continued)}

\begin{tabular}{|c|c|c|c|c|c|c|}
\hline Rank & $\begin{array}{l}\text { Agencies/Cooperatives } \\
\text { Senvice Area (Region) }\end{array}$ & Type of Ownership & Year & $\begin{array}{l}\text { Unit Cost* } \\
\text { (Cts/kwh) }\end{array}$ & $\begin{array}{r}\text { Commercial } \\
(\text { Cts/kwh) } \\
9 / 90\end{array}$ & $\begin{array}{r}\text { Industrial } \\
\text { (Cts/kwh) } \\
8 / 90\end{array}$ \\
\hline 33 & $\begin{array}{l}\text { Cheney Light Department, City of.............. } \\
\text { Spokane (NE) }\end{array}$ & Municipality & 1987 & 0.0393 & -- & -- \\
\hline 34 & $\begin{array}{l}\text { Chewelah Light Dept., City of........................ } \\
\text { Stevens (NE) }\end{array}$ & Municipality & 1988 & 0.0390 & -- & - \\
\hline 35 & $\begin{array}{l}\text { Fircrest, Town of........................................ } \\
\text { Pierce (PS) }\end{array}$ & Other & 1988 & 0.0385 & -- & -- \\
\hline 36 & $\begin{array}{l}\text { Lewis Coun; PUD............................................. } \\
\text { Lewis (SW) }\end{array}$ & Public Utility & 1989 & 0.0377 & -- & -- \\
\hline 37 & $\begin{array}{l}\text { Skamania County PUD................................. } \\
\text { Skamania (SW) }\end{array}$ & Public Utility & 1988 & 0.0376 & -- & - \\
\hline 38 & $\begin{array}{l}\text { Clark Public Utilities............................................. } \\
\text { Clark (SW) }\end{array}$ & Public Utility & 1989 & 0.0364 & 0.0350 & 0.0235 \\
\hline 39 & $\begin{array}{l}\text { McCleary Light and Power.............................. } \\
\text { Grays Harbor }(W)\end{array}$ & Municipality & 1988 & 0.0361 & - & -- \\
\hline 40 & $\begin{array}{l}\text { Benton County PUD } \\
\text { Benton (SE) }\end{array}$ & Public Utility & 1989 & 0.0356 & -- & - \\
\hline 41 & $\begin{array}{l}\text { Grays Harbor County PUD.......................... } \\
\text { Grays Harbor }(W) \\
\text { Jefferson }(W)\end{array}$ & Public Utility & 1989 & 0.0353 & - & - \\
\hline 42 & $\begin{array}{l}\text { Richland Energy Svc. Dept., Ctty of........... } \\
\text { Benton (SE) }\end{array}$ & Other & 1988 & 0.0353 & - & - \\
\hline 43 & $\begin{array}{l}\text { Elmhurst Mutual Power \& Light Co........... } \\
\text { Pierce (PS) }\end{array}$ & Cooperative & 1989 & 0.0350 & - & - \\
\hline 44 & 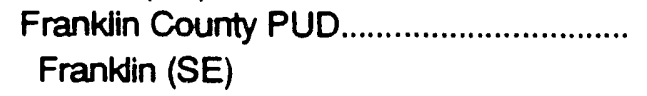 & Public Utilty & 1989 & 0.0344 & - & - \\
\hline 45 & $\begin{array}{l}\text { Vera Water and Power................................... } \\
\text { Spokane (NE) }\end{array}$ & Cooperative & 1989 & 0.0343 & - & - \\
\hline 46 & $\begin{array}{l}\text { Columbia Rural Electric Assoc., Inc........... } \\
\text { Columbia (SE) } \\
\text { Walla Walla (SE) }\end{array}$ & Cooperative & 1989 & 0.0340 & - & - \\
\hline 47 & $\begin{array}{l}\text { Parkand Light and Water Co..................... } \\
\text { Pierco (PS) }\end{array}$ & Cooperative & 1988 & 0.0328 & - & - \\
\hline 48 & $\begin{array}{l}\text { Lakeview Light and Power Co.................... } \\
\text { Pierce (PS) }\end{array}$ & Other & 1989 & 0.0326 & - & - \\
\hline 49 & $\begin{array}{l}\text { Lincoln Electric Cooperative, Inc................ } \\
\text { Uncoln (NE) }\end{array}$ & Cooperative & 1989 & 0.0326 & -- & -- \\
\hline 50 & $\begin{array}{l}\text { Seattle City Light Dept., City of...................... } \\
\text { King (PS) }\end{array}$ & Other & 1988 & 0.0312 & 0.0346 & 0.0254 \\
\hline
\end{tabular}

$-=$ Not Availebto 


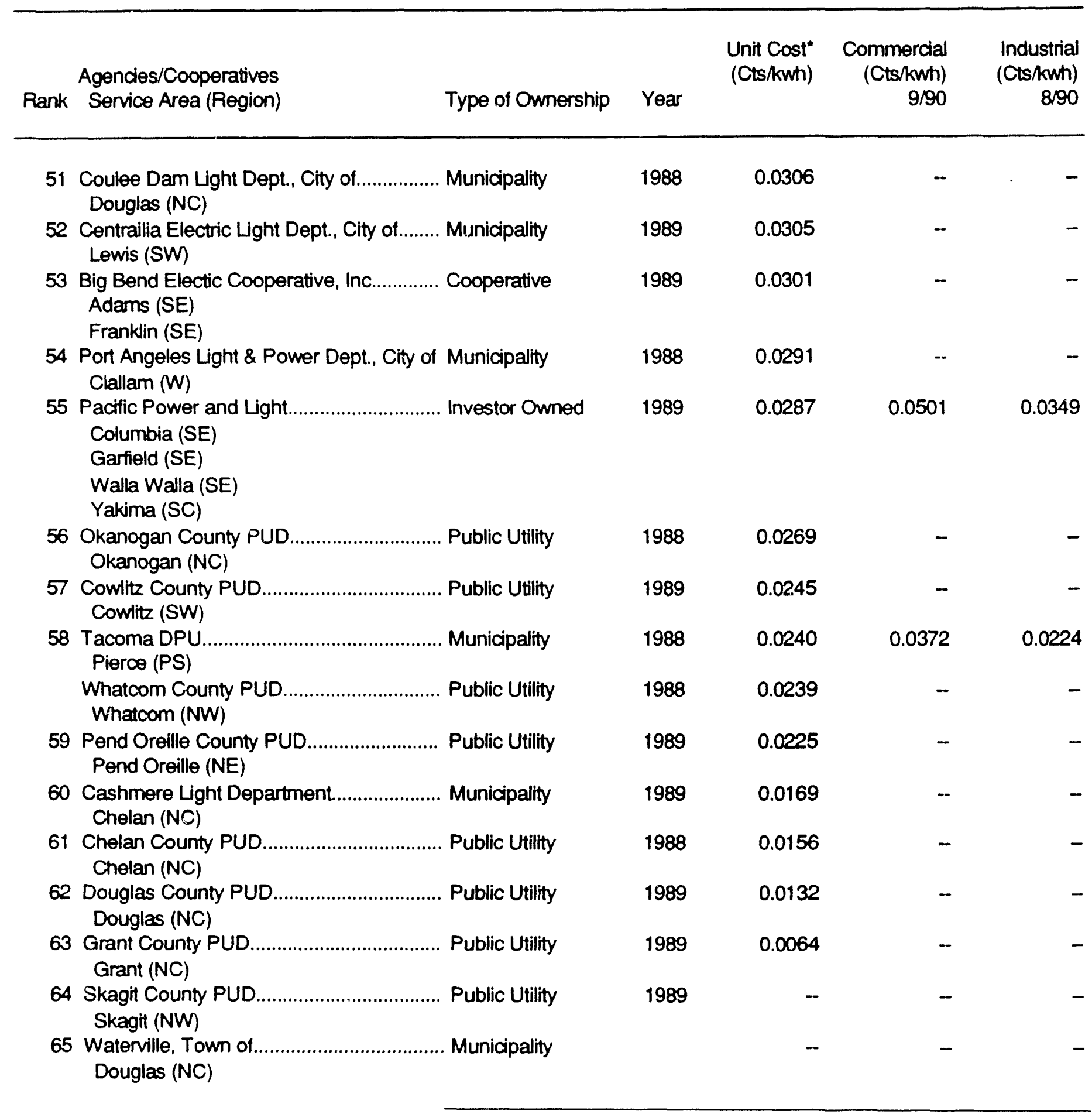

\section{- = Not Avallable}

" Unt costs were calculated by dividing dollar sales by kwti sales of the particular utility company.

Source: 1990 Northwest Electric Utility Directory, Northwest Public Power Association and Energy User News, The 
Table IV-2

Washington State Average Electric Utility Rates per Kilowatthour by Region

Sorted by Unit Costs (Cents per kWh)

\begin{tabular}{|c|c|c|c|c|c|c|}
\hline & & & & $\begin{array}{l}\text { Unit Cost* } \\
\text { (Cts/kwh) }\end{array}$ & $\begin{array}{l}\text { Commercial } \\
\text { (Cts/kwh) }\end{array}$ & $\begin{array}{l}\text { Industrial } \\
\text { (Cts/kwh) }\end{array}$ \\
\hline Region & $\begin{array}{l}\text { Agencies/Cooperatives } \\
\text { Service Area }\end{array}$ & $\begin{array}{l}\text { Type of } \\
\text { Ownership }\end{array}$ & Year & & 9/90 & $8 / 90$ \\
\hline \multirow[t]{8}{*}{ West } & $\begin{array}{l}\text { Mason County PUD No. 1......................... } \\
\text { Jefferson County } \\
\text { Mason County }\end{array}$ & Public Utility & 1988 & 0.0540 & -- & -- \\
\hline & $\begin{array}{l}\text { Puget Sound Power and Light Co.............. } \\
\text { Jefferson County }\end{array}$ & Investor Owned & 1989 & 0.0467 & 0.0529 & 0.0344 \\
\hline & $\begin{array}{l}\text { Mason County PUD No. 3................................ } \\
\text { Mason County }\end{array}$ & Public Utility & 1989 & 0.0447 & -- & -- \\
\hline & $\begin{array}{l}\text { "Clallam County PUD.................................... } \\
\text { Clallam County } \\
\text { Jefferson County }\end{array}$ & Public Utility & 1989 & 0.0439 & -- & -- \\
\hline & $\begin{array}{l}\text { Pacific County PUD No. 2.............................. } \\
\text { Pacific County }\end{array}$ & Public Utility & 1988 & 0.0424 & -- & -- \\
\hline & $\begin{array}{l}\text { McCleary Light and Power............................. } \\
\text { Grays Harbor County }\end{array}$ & Municipality & 1988 & 0.0361 & -- & -- \\
\hline & $\begin{array}{l}\text { Grays Harbor County PUD......................... } \\
\text { Grays Harbor County } \\
\text { Jefferson County }\end{array}$ & Public Utility & 1989 & 0.0353 & -- & -- \\
\hline & $\begin{array}{l}\text { Port Angeles Light \& Power Dept., City of } \\
\text { Clallam County }\end{array}$ & Municipality & 1988 & 0.0291 & - & - \\
\hline \multirow[t]{2}{*}{ Northwest } & $\begin{array}{l}\text { Orcas Power and Light Co............................ } \\
\text { San Jaun County }\end{array}$ & Cooperative & 1988 & 0.0610 & - & - \\
\hline & $\begin{array}{l}\text { Puget Sound Power and Light Co............. } \\
\text { Island County } \\
\text { Skagit County } \\
\text { Whatcom County }\end{array}$ & Investor Owned & 1989 & 0.0467 & 0.0529 & 0.0344 \\
\hline
\end{tabular}

$-=$ Not Available 
Table IV-2

Washington State Average Electric Utility Rates per Kilowatthour by Region (Continued)

\begin{tabular}{|c|c|c|c|c|c|c|}
\hline \multirow[b]{2}{*}{ Region } & \multirow[b]{2}{*}{$\begin{array}{l}\text { Agencies/Cooperatives } \\
\text { Service Area }\end{array}$} & \multirow[b]{2}{*}{$\begin{array}{l}\text { Type of } \\
\text { Ownership }\end{array}$} & \multirow[b]{2}{*}{ Year } & \multirow[t]{2}{*}{$\begin{array}{l}\text { Unit Cost* } \\
\text { (Cts/kwh) }\end{array}$} & \multirow{2}{*}{$\begin{array}{r}\text { Commercial } \\
\text { (Cts/kwh) } \\
9 / 90\end{array}$} & \multirow{2}{*}{$\begin{array}{r}\text { Industrial } \\
(\mathrm{Cts} / \mathrm{kWh}) \\
8 / 90\end{array}$} \\
\hline & & & & & & \\
\hline \multirow[t]{5}{*}{$\begin{array}{l}\text { Northwest } \\
\text { (continued) }\end{array}$} & $\begin{array}{l}\text { Sumas, City of } \\
\text { Whatcom County }\end{array}$ & Municipality & 1989 & 0.0436 & -- & -- \\
\hline & $\begin{array}{l}\text { Blaine City Light............................................... } \\
\text { Whatcom County }\end{array}$ & Municipality & 1988 & 0.0404 & -- & -- \\
\hline & $\begin{array}{l}\text { Snohomish County PUD................................ } \\
\text { Island County }\end{array}$ & Public Utility & 1988 & 0.0402 & -- & - \\
\hline & $\begin{array}{l}\text { Whatcom County PUD.................................. } \\
\text { Whatcom County }\end{array}$ & Public Utility & 1988 & 0.0239 & -- & - \\
\hline & $\begin{array}{l}\text { Skagit County PUD } \\
\text { Skagit County }\end{array}$ & Public Utility & 1989 & - & - & -- \\
\hline \multirow[t]{7}{*}{ Puget Sound } & $\begin{array}{l}\text { Tanner Electric Cooperative............................ } \\
\text { King County }\end{array}$ & Cooperative & 1988 & 0.0546 & -- & -- \\
\hline & $\begin{array}{l}\text { Peninsula Light Co......................................... } \\
\text { Pierce County }\end{array}$ & Cooperative & 1989 & 0.0471 & - & -- \\
\hline & $\begin{array}{l}\text { Puget Sound Power \& Light Co................. } \\
\text { Kitsap County } \\
\text { Pierce County } \\
\text { Thurston County } \\
\text { King County }\end{array}$ & Investor Owned & 1989 & 0.0467 & 0.0529 & 0.0344 \\
\hline & $\begin{array}{l}\text { OHOP Mutual Light Co........................................ } \\
\text { Pierce County }\end{array}$ & Cooperative & 1988 & 0.0449 & - & - \\
\hline & $\begin{array}{l}\text { Alder Mutual Light Co....................................... } \\
\text { Pierce County }\end{array}$ & Cooperative & 1988 & 0.0440 & -- & -- \\
\hline & $\begin{array}{l}\text { Eatonville Power \& Light Co............................. } \\
\text { Pierce County }\end{array}$ & Municipality & 1988 & 0.0423 & - & - \\
\hline & $\begin{array}{l}\text { Steilacoom, Town of.......................................... } \\
\text { Pierce County }\end{array}$ & Other & 1988 & 0.0417 & -- & -- \\
\hline
\end{tabular}

$-=$ Not Available 
Table IV-2

\section{Washington State Average Electric Utility Rates per Kilowatthour by Region (Continued)}

\begin{tabular}{|c|c|c|c|c|c|c|}
\hline \multirow[b]{2}{*}{ Region } & \multirow[b]{2}{*}{$\begin{array}{l}\text { Agencies/Cooperatives } \\
\text { Service Area }\end{array}$} & \multirow[b]{2}{*}{$\begin{array}{l}\text { Type of } \\
\text { Ownership }\end{array}$} & \multirow[b]{2}{*}{ Year } & \multirow[t]{2}{*}{$\begin{array}{l}\text { Unit Cost" } \\
\text { (Cts/kwh) }\end{array}$} & \multirow{2}{*}{$\begin{array}{r}\text { Commercial } \\
\text { (Cts/kwh) } \\
9 / 90\end{array}$} & \multirow{2}{*}{$\begin{array}{r}\text { Industrial } \\
\text { (Cts/kwh) } \\
8 / 90\end{array}$} \\
\hline & & & & & & \\
\hline \multirow[t]{9}{*}{$\begin{array}{l}\text { Puget Sound } \\
\text { (continued) }\end{array}$} & $\begin{array}{l}\text { Milton, City of...................................................... } \\
\text { Pierve County }\end{array}$ & Municipality & 1988 & 0.0411 & - & -- \\
\hline & $\begin{array}{l}\text { Snohomish County PUD......................................... } \\
\text { Snohomish County }\end{array}$ & Public Utility & 1988 & 0.0402 & 0.0342 & 0.0272 \\
\hline & 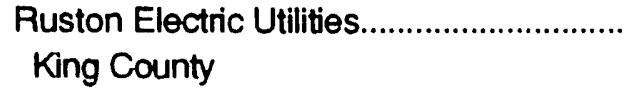 & Cooperative & 1989 & 0.0398 & -- & -- \\
\hline & $\begin{array}{l}\text { Fircrest, Town of............................................ } \\
\text { Pierce County }\end{array}$ & Other & 1988 & 0.0385 & - & -- \\
\hline & $\begin{array}{l}\text { Elmhurst Mutual Power \& Light Co............. } \\
\text { Pierce County }\end{array}$ & Cooperative & 1989 & 0.0350 & -- & -- \\
\hline & $\begin{array}{l}\text { Parkland Light and Water Co......................... } \\
\text { Pierce County }\end{array}$ & Cooperative & 1988 & 0.0328 & -- & - \\
\hline & $\begin{array}{l}\text { Lakeview Light and Power Co........................ } \\
\text { Pierce County }\end{array}$ & Other & 1989 & 0.0326 & - & - \\
\hline & $\begin{array}{l}\text { Seattle City Light Dept., City of.................... } \\
\text { King County }\end{array}$ & Other & 1988 & 0.0312 & 0.0346 & 0.0254 \\
\hline & $\begin{array}{l}\text { Tacoma DPU................................................. } \\
\text { Pierce County }\end{array}$ & Municipality & 1988 & 0.0240 & 0.0372 & 0.0224 \\
\hline \multirow[t]{3}{*}{ Southwest } & $\begin{array}{l}\text { Wahkiakum County PUD............................... } \\
\text { Wahkiakum County }\end{array}$ & Public Utility & 1988 & 0.0477 & - & - \\
\hline & $\begin{array}{l}\text { Lewis Couny PUD } \\
\text { Lewis County }\end{array}$ & Public Utility & 1989 & 0.0377 & - & - \\
\hline & $\begin{array}{l}\text { Skamania County PUD................................... } \\
\text { Skamania County }\end{array}$ & Public Utility & 1988 & 0.0376 & - & - \\
\hline
\end{tabular}

- = Not Avaitable 
Table IV-2

Washington State Average Electric Utility Rates per Kilowatthour by Region (Continued)

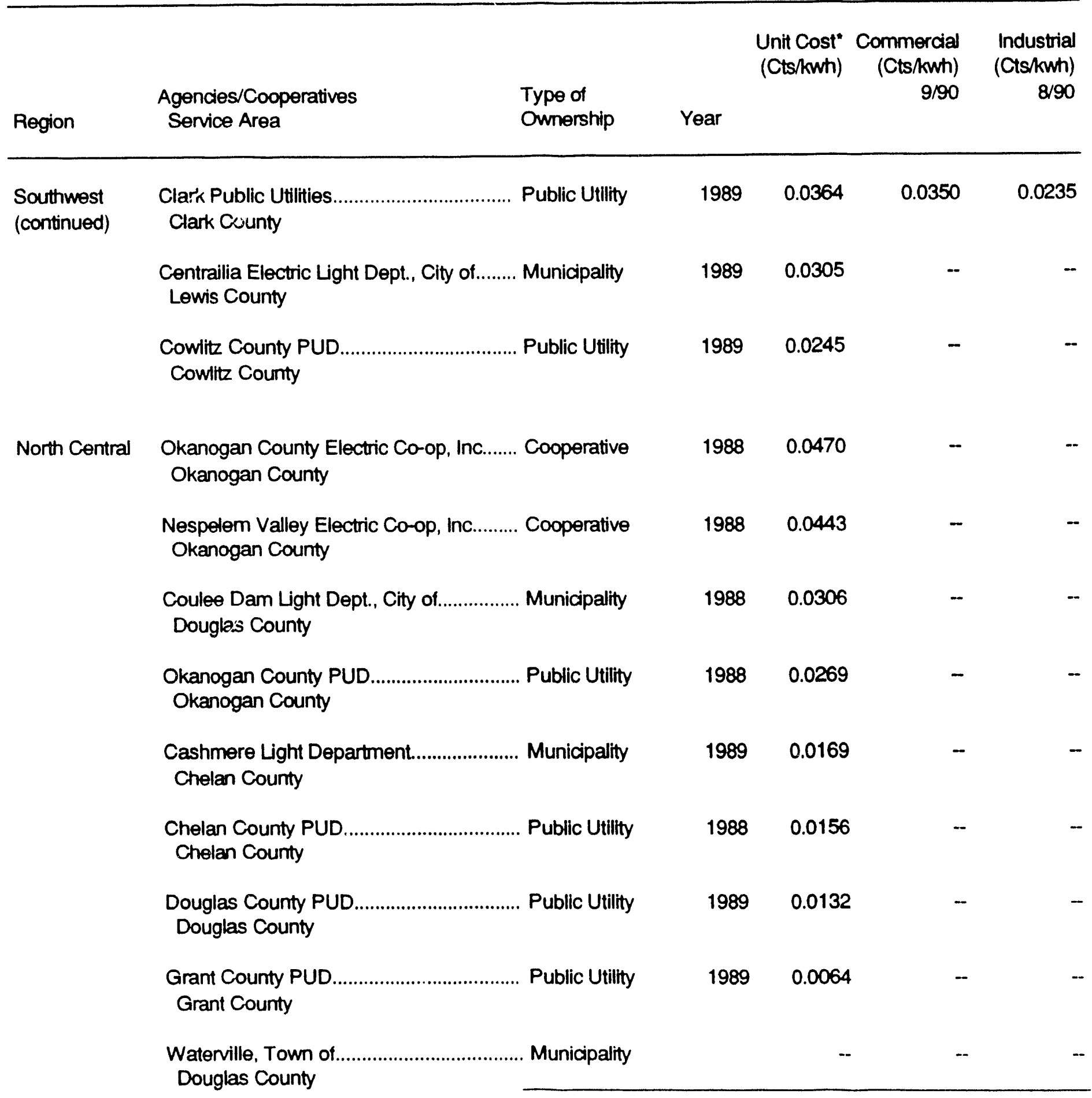

- $=$ Not Available 
Table IV-2

\section{Washington State Average Electric Utility Rates per Kilowatthour by Region (Continued)}

\begin{tabular}{|c|c|c|c|c|c|}
\hline \multirow[b]{2}{*}{ Region } & \multirow[b]{2}{*}{$\begin{array}{l}\text { Agencies/Cooperatives } \\
\text { Service Area }\end{array}$} & \multirow[b]{2}{*}{ Year } & \multirow{2}{*}{$\begin{array}{l}\text { Unit Cost* } \\
\text { (Cts/kwh) }\end{array}$} & \multirow{2}{*}{$\begin{array}{r}\text { Commercial } \\
(\text { Cts/kwh) } \\
9 / 90\end{array}$} & \multirow{2}{*}{$\begin{array}{r}\text { Industrial } \\
\text { (Cts/kwh) } \\
8 / 90\end{array}$} \\
\hline & & & & & \\
\hline \multirow[t]{6}{*}{ South Central } & $\begin{array}{l}\text { Puget Sound Power and Light Co............. Investor Owned } \\
\text { Kittitas County }\end{array}$ & 1989 & 0.0467 & 0.0529 & 0.0344 \\
\hline & $\begin{array}{l}\text { Kilttitas County PUD..................................... Public Utility } \\
\text { Kittitas County }\end{array}$ & 1988 & 0.0466 & - & -- \\
\hline & $\begin{array}{l}\text { Benton Rural Electric Assoc., Inc............... Cooperative } \\
\text { Yakima County }\end{array}$ & 1988 & 0.0428 & - & - \\
\hline & $\begin{array}{l}\text { Klickitat County PUD.................................... Public Utility } \\
\text { Klickitat County }\end{array}$ & 1989 & 0.0417 & -- & -- \\
\hline & $\begin{array}{l}\text { Ellensberg, City of............................................. Municipality } \\
\text { Kitttitas County }\end{array}$ & 1988 & 0.0402 & -- & -- \\
\hline & $\begin{array}{l}\text { Pacific Power and Light................................ Investor Owned } \\
\text { Yakima County }\end{array}$ & 1988 & 0.0287 & 0.0501 & 0.0349 \\
\hline \multirow[t]{5}{*}{ Northeast } & $\begin{array}{l}\text { Kootenal Electric Cooperative...................... Cooperative } \\
\text { Spokane County }\end{array}$ & & 0.0512 & - & - \\
\hline & $\begin{array}{l}\text { Northem Lights, Inc....................................... Cooperative } \\
\text { Pend Oreille County }\end{array}$ & & 0.0486 & -- & -- \\
\hline & $\begin{array}{l}\text { Inland Power \& Light Company.................. Cooperative } \\
\text { Pend Oreille County } \\
\text { Spokane County }\end{array}$ & 1989 & 0.0482 & -- & -- \\
\hline & $\begin{array}{l}\text { Ferry County PUD....................................... Public Utility } \\
\text { Ferry County }\end{array}$ & 1989 & 0.0479 & - & - \\
\hline & $\begin{array}{l}\text { Nespelem Valley Electric Co-op, Inc.......... Cooperative } \\
\text { Femy County }\end{array}$ & 1988 & 0.0443 & - & - \\
\hline
\end{tabular}

- = Not Avaliable 
Table IV-2

\section{Washington State Average Electric Utility Rates per Kilowatthour by Region (Continued)}

\begin{tabular}{|c|c|c|c|c|c|c|}
\hline \multirow[b]{2}{*}{ Region } & \multirow[b]{2}{*}{$\begin{array}{l}\text { Agencies/Cooperatives } \\
\text { Service Area }\end{array}$} & \multirow[b]{2}{*}{$\begin{array}{l}\text { Type of } \\
\text { Ownership }\end{array}$} & \multirow[b]{2}{*}{ Year } & \multirow[t]{2}{*}{$\begin{array}{l}\text { Unit Cost* } \\
\text { (Cts/kwh) }\end{array}$} & \multirow{2}{*}{$\begin{array}{r}\text { Commercial } \\
\text { (Cts/kwh) } \\
9 / 90\end{array}$} & \multirow{2}{*}{$\begin{array}{r}\text { Industrial } \\
\text { (Cts/kwh) } \\
8 / 90\end{array}$} \\
\hline & & & & & & \\
\hline \multirow[t]{7}{*}{$\begin{array}{l}\text { Northeast } \\
\text { (continued) }\end{array}$} & $\begin{array}{l}\text { Modem Electic Co............................................ } \\
\text { Spokane County }\end{array}$ & Cooperative & 1988 & 0.0411 & -- & -- \\
\hline & $\begin{array}{l}\text { Washington Water Power Co., The............. } \\
\text { Spokane County }\end{array}$ & Investor Owned & 1988 & 0.0399 & 0.0520 & 0.0347 \\
\hline & $\begin{array}{l}\text { Cheney Light Department, Clty of............... } \\
\text { Spokane County }\end{array}$ & Municipality & 1987 & 0.0393 & -- & -- \\
\hline & $\begin{array}{l}\text { Chewelah Light Dept., City of....................... } \\
\text { Stevens County }\end{array}$ & Municipality & 1988 & 0.0390 & -- & -- \\
\hline & $\begin{array}{l}\text { Vera Water and Power.................................... } \\
\text { Spokane County }\end{array}$ & Cooperative & 1989 & 0.0343 & -- & - \\
\hline & $\begin{array}{l}\text { Lincoln Electric Cooperative, Inc................. } \\
\text { Lincoln County }\end{array}$ & Cooperative & 1989 & 0.0326 & -- & -- \\
\hline & $\begin{array}{l}\text { Pend Oreille County PUD............................ } \\
\text { Pend Oreille County }\end{array}$ & Public Utility & 1989 & 0.0225 & -- & - \\
\hline \multirow[t]{4}{*}{ Southeast } & $\begin{array}{l}\text { Clearwater Power Co................................... } \\
\text { Asotin County } \\
\text { Whitman County }\end{array}$ & Cooperative & & 0.0630 & - & - \\
\hline & $\begin{array}{l}\text { Inland Power \& Light Co.............................. } \\
\text { Garfield County } \\
\text { Whitman County }\end{array}$ & Cooperative & 1989 & 0.0482 & - & -- \\
\hline & $\begin{array}{l}\text { Benton Rural Electric Assoc., Inc................ } \\
\text { Benton County }\end{array}$ & Cooperative & 1988 & 0.0428 & - & - \\
\hline & 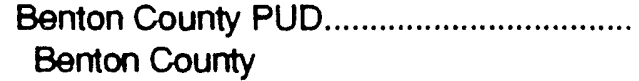 & Public Utility & 1989 & 0.0356 & - & -- \\
\hline
\end{tabular}

- = Not Avallable 
Table IV-2

Washington State Average Electric Utility Rates per Kilowatthour by Region (Continued)

\begin{tabular}{|c|c|c|c|c|c|c|}
\hline \multirow[b]{2}{*}{ Region } & \multirow[b]{2}{*}{$\begin{array}{l}\text { Agencies/Cooperatives } \\
\text { Service Area }\end{array}$} & \multirow[b]{2}{*}{$\begin{array}{l}\text { Type of } \\
\text { Ownership }\end{array}$} & \multirow[b]{2}{*}{ Year } & \multirow[t]{2}{*}{$\begin{array}{l}\text { Untt Cost" } \\
\text { (Cts/kwh) }\end{array}$} & \multirow{2}{*}{$\begin{array}{r}\text { Commercial } \\
\text { (Cts/kwh) } \\
9 / 90\end{array}$} & \multirow{2}{*}{$\begin{array}{r}\text { Industrial } \\
\text { (Cts/kwh) } \\
8 / 90\end{array}$} \\
\hline & & & & & & \\
\hline \multirow[t]{5}{*}{$\begin{array}{l}\text { Southeast } \\
\text { (continued) }\end{array}$} & $\begin{array}{l}\text { Richland Energy Service Dept., City of..... } \\
\text { Benton County }\end{array}$ & Other & 1988 & 0.0353 & - & - \\
\hline & $\begin{array}{l}\text { Franklin County PUD } \\
\text { Franklin County }\end{array}$ & Public Utility & 1989 & 0.0344 & -- & -. \\
\hline & $\begin{array}{l}\text { Columbia Rural Electric Assoc., Inc........... } \\
\text { Columbia County } \\
\text { Walla Walla County }\end{array}$ & Cooperative & 1989 & 0.0340 & -- & -- \\
\hline & $\begin{array}{l}\text { Big Bend Electic Cooperative, Inc............... } \\
\text { Adams County } \\
\text { Franklin County }\end{array}$ & Cooperative & 1989 & 0.0301 & - & - \\
\hline & $\begin{array}{l}\text { Pacific Power and Light............................... } \\
\text { Columbia County } \\
\text { Garfield County } \\
\text { Walla Walla County }\end{array}$ & Investor Owned & 1989 & 0.0287 & 0.0501 & 0.0349 \\
\hline
\end{tabular}

$-=$ Not Available

- Unit costs were calculated by dividing dollar sales by kwh sales of the particular utility company.

Source: 1990 Northwest Electric Utility Directory, Northwest Public Power Association, and Energy User News, The Chilton Company 
Table IV-3

Net Generation, Fuel Consumption, and Fuel Stock by Company and Plant

\begin{tabular}{|c|c|c|c|c|c|c|c|c|c|c|c|}
\hline \multirow[b]{2}{*}{$\begin{array}{l}\text { Company } \\
\text { Plant }\end{array}$} & \multirow[b]{2}{*}{ Coal } & \multicolumn{5}{|c|}{ (thousand kilowatthours) } & \multicolumn{2}{|c|}{$\begin{array}{l}\text { Consumption } \\
\text { (thousands) }\end{array}$} & \multicolumn{3}{|c|}{ (thousands) } \\
\hline & & $\begin{array}{l}\text { Petro- } \\
\text { leum }\end{array}$ & Gas & Hydro & Nuclear & Other"* & $\begin{array}{l}\text { Coal } \\
\text { (short } \\
\text { tons) }\end{array}$ & $\begin{array}{l}\text { Petro- } \\
\text { leum } \\
\text { (bbls) }\end{array}$ & Gas & $\begin{array}{l}\text { Coal } \\
\text { (short } \\
\text { tons) }\end{array}$ & $\begin{array}{l}\text { Petro- } \\
\text { leum } \\
\text { (bbls) }\end{array}$ \\
\hline Chelan Co. PUD.... & -- & -- & -- & 779,415 & - & -. & -- & -- & -- & - & -- \\
\hline Chelan & -- & - & - & 38,147 & -- & -- & -. & -- & -- & - & -- \\
\hline Rock Island & -. & -- & - & 233,989 & - & -- & -- & - & - & - & - \\
\hline Rocky Reach & -- & -- & -- & 517,279 & -- & -- & -- & -- & -- & - & -- \\
\hline Grant Co. PUD........ & - & -- & -- & 840,782 & -- & -- & -. & -- & -- & - & -- \\
\hline Pec Hdwks & -- & -- & -- & -- & - & -- & -- & -- & -- & - & -- \\
\hline Priest Rapids & - & - & -- & 435,871 & - & -- & -. & -- & - & - & -- \\
\hline Quincy Chut & - & - & - & - & - & -- & - & -- & -- & - & -- \\
\hline Wanapum & - & -- & -- & 404,911 & -- & -- & -- & -- & -- & - & -- \\
\hline Orcas P\&L.... & - & -- & - & - & - & - & - & - & - & - & -- \\
\hline Eastsound & -- & - & - & -- & - & - & - & -- & - & - & -- \\
\hline Pacificorp.................. & 533,779 & 429 & - & 104,116 & - & - & 373 & 1 & 1,470 & 3 & -- \\
\hline Centralia & 533,779 & 429 & -- & -- & - & -- & 373 & 1 & 1,470 & 3 & - \\
\hline Naches Drop & - & - & - & 557 & - & - & - & -- & -- & - & - \\
\hline Switt No. 2 & - & - & - & 28,259 & - & - & - & - & - & - & - \\
\hline Yale & - & - & - & 75,300 & - & -- & - & - & - & - & - \\
\hline Pend Oreille PUD & - & - & - & 50,451 & - & - & - & - & - & - & - \\
\hline Box Canyon & - & - & - & 50,451 & - & - & - & - & - & - & -- \\
\hline Calispel Creok & -- & - & -- & -- & - & -- & - & - & -- & - & -- \\
\hline Placer Co. Wtr Ag.. & - & - & - & 212 & - & - & - & - & - & - & -- \\
\hline Hell Hole & - & - & - & 212 & -- & - & - & - & -- & - & - \\
\hline Puget Sound P\&L & - & 74 & 175 & 172,839 & - & - & - & - & 2 & - & 205 \\
\hline Crystal Mountain & - & 27 & - & - & - & - & - & - & - & - & 1 \\
\hline Electron & - & - & -- & 11,786 & - & -- & - & - & -- & - & \\
\hline Frederickson & - & 61 & 76 & - & - & - & - & * & 1 & - & 95 \\
\hline Fredonia & -- & - & - & - & - & -- & -- & - & -- & - & 50 \\
\hline
\end{tabular}

- Number less than 0.5 rounded to zero.

* Oher sources inctude geothermal,solar, wood, wind, and waste. 
Table IV-3

Net Generation, Fuel Consumption, and Fuel Stock of by Company and Plant (Continued)

\begin{tabular}{|c|c|c|c|c|c|c|c|c|c|c|c|}
\hline \multirow[b]{2}{*}{$\begin{array}{l}\text { Company } \\
\text { Plant }\end{array}$} & \multirow[b]{2}{*}{ Coal } & \multicolumn{5}{|c|}{ (thousand kilowatthours) } & \multicolumn{2}{|c|}{$\begin{array}{l}\text {-Consumption } \\
\text { (thousand) }\end{array}$} & \multicolumn{3}{|c|}{ (thousands) } \\
\hline & & leum & Gas & Hydro & Nuclear & Other"* & $\begin{array}{l}\text { Coal } \\
\text { (short } \\
\text { tons) }\end{array}$ & $\begin{array}{l}\text { leum } \\
\text { (bbls) }\end{array}$ & Gas & $\begin{array}{l}\text { Coal } \\
\text { (short } \\
\text { tons) }\end{array}$ & lour \\
\hline Puget Sound P\&L (C & & & & & & & & & & & \\
\hline Lower Baker & - & -- & - & 47,788 & -- & -- & - & -- & - & - & \\
\hline Nooksack & -- & -- & -- & 514 & -- & -- & -- & -- & -- & - & \\
\hline Shuffleton & -- & (58) & -- & -- & -- & -- & -- & • & -- & - & \\
\hline Snoqualmie & -- & -- & -- & 26,874 & - & -- & -- & -- & - & - & \\
\hline South Widbey & - & 15 & -- & - & - & - & - & $\cdot$ & -- & - & \\
\hline Upper Baker & - & -- & -- & 56,707 & -- & -- & -- & -- & -- & - & \\
\hline White River & - & - & -- & 29,170 & - & -- & -- & -- & -- & - & 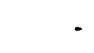 \\
\hline Whitehom & - & 29 & 99 & - & -- & -- & -- & • & 1 & - & 5 \\
\hline Seattle Light Dept. & -- & - & - & 837,351 & - & - & - & -- & -- & - & \\
\hline Boundary & - & -- & -- & 374,493 & - & -- & - & -- & -- & - & \\
\hline Codar Falls & - & -- & - & 17,816 & - & - & -. & -- & - & - & \\
\hline Diablo & -- & -- & -- & 110,893 & - & -- & -- & -- & -- & - & \\
\hline Gorge & -- & - & - & 121,447 & - & - & - & -- & - & - & \\
\hline New Halem & - & - & - & 1,261 & - & - & - & -- & - & - & \\
\hline Ross Dam & - & - & - & 211,441 & - & -- & - & -- & - & - & \\
\hline Spokane, City of.... & - & - & - & - & -- & -- & - & - & -- & - & 1 \\
\hline Upriver & -- & - & -- & - & - & -- & -- & -- & -- & - & 1 \\
\hline Tacoma PUD.......... & - & - & - & 369,114 & -- & - & - & - & - & - & \\
\hline Alder & -- & -- & -- & 26,486 & - & - & - & -- & - & - & \\
\hline Cushman 1 & - & - & - & 33,874 & - & - & - & - & - & - & \\
\hline Cushman 2 & - & - & - & 11,847 & - & - & - & - & - & - & \\
\hline La Grande & - & - & - & 30,385 & - & -- & - & - & - & - & \\
\hline Mayfield & - & - & -- & 85,595 & -. & -- & -- & - & - & - & \\
\hline Mossyrock & - & - & - & 180,927 & -- & - & -- & - & - & - & \\
\hline Usbia Wapato Irr. .. & -- & - & - & -- & -- & -- & - & -- & -- & - & \\
\hline Drop 2 & - & - & - & - & -- & - & -- & - & - & - & \\
\hline Drop 3 & - & - & - & - & - & - & -. & - & -- & - & \\
\hline
\end{tabular}

- Number less than 0.5 rounded to zero

- Other sources inchude geothermal,solar, wood, wind, and waste. 
Table IV-3

Net Generation, Fuel Consumption, and Fuel Stock of by Company and Plant (Continued)

$\begin{array}{lll}\text { (thousand kilowatthours) } & \begin{array}{c}\text { Consumption } \\ \text { (thousand) }\end{array} & \text { (thousands) }\end{array}$

\begin{tabular}{|c|c|c|c|c|c|c|c|c|c|c|}
\hline $\begin{array}{l}\text { Company } \\
\text { Plant }\end{array}$ & Coal & $\begin{array}{l}\text { Petro- } \\
\text { leum }\end{array}$ & Gas & Hydro & Nuclear & Other"* & $\begin{array}{l}\text { Coal } \\
\text { (short } \\
\text { tons) }\end{array}$ & $\begin{array}{l}\text { Petro- } \\
\text { leum } \\
\text { (bbls) }\end{array}$ & Gas & $\begin{array}{l}\text { Coal } \\
\text { (short } \\
\text { tons) }\end{array}$ \\
\hline
\end{tabular}

\begin{tabular}{|c|c|c|c|c|c|c|c|c|c|c|c|}
\hline USBR PacNW Reg & - & - & - & -- & -- & - & - & - & -- & - & - \\
\hline Chandler & -- & - & -- & - & - & -- & -- & -- & -- & - & -- \\
\hline Grand Coulee & -- & - & -- & (35) & -- & -- & -- & - & -- & - & -- \\
\hline Roza & -- & - & - & 1,244 & - & -- & - & - & - & - & -- \\
\hline USCE - N.Pac. Div. & - & -- & -- & $2,077,452$ & - & - & - & -- & -- & - & - \\
\hline Chief Joesph & -- & -- & -- & 934,777 & -- & - & -- & -- & -- & - & -- \\
\hline Dalles & -- & - & - & 648,995 & - & -- & -- & -- & -- & - & -- \\
\hline Ico Harbor & -- & -- & -- & 125,699 & -- & -- & -- & - & -- & - & -- \\
\hline Little Goose & - & - & -- & 120,764 & -- & - & -- & - & -- & - & -- \\
\hline Lower Granite & - & -- & - & 121,591 & - & - & - & -- & -- & - & - \\
\hline Lower Monument & - & - & - & 125,626 & - & - & - & -- & -- & - & -- \\
\hline 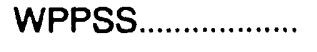 & -- & - & - & - & 489,999 & - & - & -- & - & - & -- \\
\hline Hantord & -- & -- & - & - & - & - & - & - & -- & - & -- \\
\hline Packwood & - & - & - & - & - & - & - & - & -- & - & -- \\
\hline WNP-2 & - & - & - & - & 489,999 & - & - & - & - & - & - \\
\hline WA Wt PWr Co. .... & -- & -- & -- & 66,989 & - & 28,324 & - & -- & * & - & - \\
\hline Kettle Falls & - & -- & -- & - & - & 28,324 & - & - & " & - & $\cdots$ \\
\hline Little Falls & - & - & - & 15,110 & - & - & - & - & - & - & - \\
\hline Long Lake & - & - & - & 35,609 & - & - & - & - & - & - & $\cdot$ \\
\hline Meyers Falls & - & - & - & 591 & - & - & - & - & - & - & - \\
\hline Monroe Street & - & - & -- & - & - & - & - & - & - & - & - \\
\hline Nine Mile & - & - & - & 8,925 & - & - & - & - & - & - & - \\
\hline Northeast & - & - & - & -- & - & - & - & -- & - & - & $\cdots$ \\
\hline Upper Falls & - & - & -- & 6,754 & - & - & - & - & - & - & - \\
\hline
\end{tabular}

- = Number less than 0.5 rounded to zero.

- Other sources inchude geothermal,solar, wood, wind, and waste.

Source: Energy Information Administration, "Electric Power Monthly," March 1991 
Table IV-4

Natural and Other Gases Produced and Purchased by Natural Gas Pipeline Companies, October 1990

(Million Cubic Feet)

\begin{tabular}{|c|c|c|c|c|c|c|c|c|}
\hline Pipeline Company & $\begin{array}{l}\text { Trans. } \\
\text { ported } \\
\text { Gas }\end{array}$ & $\begin{array}{r}\text { Natural } \\
\text { Gas } \\
\text { Production }\end{array}$ & $\begin{array}{l}\text { Manufactured, } \\
\text { Liquefied Nat., } \\
\text { Gassified Coal } \\
\text { and Synthetic } \\
\text { Gas Production }\end{array}$ & $\begin{array}{r}\text { From } \\
\text { Producer }\end{array}$ & $\begin{array}{r}\text { Intra- } \\
\text { company } \\
\text { Transfers }\end{array}$ & Imports & $\begin{array}{l}\text { From } \\
\text { Others }\end{array}$ & Total \\
\hline Northwest & 62,480 & (3) * & 0 & 1,465 & (3) * & 3,467 & 0 & 4,929 \\
\hline Pacific Gas Transm & 13,185 & 0 & 0 & 174 & 0 & 32,259 & 0 & 32,433 \\
\hline
\end{tabular}

- Indudes out-of-period adjustments to correct data in prior month.

Sourœ: Energy Information Administration, Natural Gas Monthly, January 1991; Form FERC-11 


\section{Monthly Natural Gas Commercial and Industrial Utility Rates}

\begin{tabular}{|c|c|c|c|c|}
\hline $\begin{array}{l}\text { Gas Company } \\
\text { County (Region) }\end{array}$ & Type of Service & $\begin{array}{l}\text { Charge } \\
\text { Per } \\
\text { Therm* } \\
\text { (Cents) }\end{array}$ & $\begin{array}{l}\text { Number } \\
\text { of } \\
\text { Therms } \\
\text { Per Month }\end{array}$ & $\begin{array}{r}\text { Effective } \\
\text { Date }\end{array}$ \\
\hline \multicolumn{5}{|c|}{ Cascade Natural Gas } \\
\hline & Gerivial Commercial & 0.5001 & First 50 & $(03-12-90)$ \\
\hline & General Industrial & 0.4424 & First 1,000 & $(03-12-90)$ \\
\hline \multicolumn{5}{|c|}{ Northwest Natural Gas } \\
\hline & Commercial \& Industrial & 0.5753 & First 10 & $(10-01-90)$ \\
\hline & Firm - High Load Factor & 0.4213 & First 500 & $(10-01-90)$ \\
\hline & Large Firm & 0.4574 & First 4,000 & $(10-01-90)$ \\
\hline \multicolumn{5}{|c|}{ Washington Natural Gas } \\
\hline & Commercial \& Industrial General (Optional) & 0.5820 & First 100 & $(06-01-88)$ \\
\hline & $\begin{array}{l}\text { Large General-Firm } \\
\text { High Annual Load Factor Large General-Firm }\end{array}$ & $\begin{array}{l}0.3666 \\
0.3567\end{array}$ & $\begin{array}{l}\text { First } 200 \\
\text { First } 500\end{array}$ & $\begin{array}{l}(08-01-90) \\
(08-01-90)\end{array}$ \\
\hline \multicolumn{5}{|c|}{ Washington Water Power } \\
\hline & General - Firm & 0.3762 & First 1 & $(08-01-90)$ \\
\hline & High Annual Load Factor Large General-Firm & 0.3567 & First 500 & $(08-01-90)$ \\
\hline
\end{tabular}

- Charge per therm decrease as the number of therms consumed incre 3 ses.

Note:

Cascade Natural Gas sorvos: Adams (SE). Benton (SE), Chelan (NC), Ctark (SW), Cowiz (SW), Douglas (NC), Frankin (SE), Grant (NK), Grays Harbor (W). Istand (NW), Kisap (PS), Mason (W). Skagk (NW) Snohomish (PS), Walla Walla (SW), Whatoom (NW), and Yakima (SC) Countios.

Northwest Natural Gas serves: Kickitat (SC), Skamania (SW), and Clark (SW) Counties.

Wastington Natural Gas serves: King (PS). Lewis (SW), Snchomish (PS), Thurston (PS), and Pierce (PS) Counties.

Washington Water Power serves: Adams (SE), Asotin (SE), Fermy (NE), Frankin (SE), Garfield (SE), Grant (NC), Kickitat (SC), Linooh (NE), Spokane (NE), Stevens (NE), and Whitman (SE) Counties.

Source: Washington State Utilities and Transportation Commission. 
Table IV-6

Washington State Coal Consumption and Prices By' Sector Consumption and Price

\begin{tabular}{|c|c|c|c|c|c|c|c|c|c|c|c|c|c|c|c|c|}
\hline \multirow[b]{2}{*}{ Year } & \multicolumn{4}{|c|}{ Residential___ } & \multicolumn{4}{|c|}{ _Commercial_____- } & \multicolumn{4}{|c|}{ - Industrial-_ } & \multicolumn{4}{|c|}{-Electric Utilities--... } \\
\hline & $\begin{array}{l}\text { Billion } \\
\text { Btu }\end{array}$ & MBtu & $\begin{array}{l}\text { Thous } \\
\text { Short } \\
\text { Tons }\end{array}$ & $\begin{array}{l}\$ \\
\text { Short } \\
\text { Tons }\end{array}$ & $\begin{array}{l}\text { Billion } \\
\text { Btu }\end{array}$ & MBtu & $\begin{array}{l}\text { Thous } \\
\text { Short } \\
\text { Tons }\end{array}$ & $\begin{array}{l}\$ \\
\text { Short } \\
\text { Tons }\end{array}$ & $\begin{array}{l}\text { Billion } \\
\text { Btu }\end{array}$ & M Btu & $\begin{array}{l}\text { Thous } \\
\text { Short } \\
\text { Tons }\end{array}$ & $\begin{array}{l}\$ 1 \\
\text { Short } \\
\text { Tons }\end{array}$ & $\begin{array}{c}\text { Billion } \\
\text { Bttu }\end{array}$ & MBtu & $\begin{array}{l}\text { Thous } \\
\text { Short } \\
\text { Tons }\end{array}$ & $\begin{array}{l}\$ \\
\text { Short } \\
\text { Tons }\end{array}$ \\
\hline $\begin{array}{l}1960 \\
1961\end{array}$ & $\begin{array}{l}1,424 \\
1,605\end{array}$ & $\begin{array}{l}\text { NA } \\
\text { NA }\end{array}$ & $\begin{array}{l}63 \\
71\end{array}$ & $\begin{array}{l}\text { NA } \\
\text { NA }\end{array}$ & $\begin{array}{l}2,644 \\
2,983\end{array}$ & $\begin{array}{l}\text { NA } \\
\text { NA }\end{array}$ & $\begin{array}{l}117 \\
132\end{array}$ & $\begin{array}{l}\text { NA } \\
\text { NA }\end{array}$ & $\begin{array}{l}9,492 \\
9,582\end{array}$ & $\begin{array}{l}\text { NA } \\
\text { NA }\end{array}$ & $\begin{array}{l}420 \\
424\end{array}$ & $\begin{array}{l}\text { NA } \\
\text { NA }\end{array}$ & $\begin{array}{l}0 \\
0\end{array}$ & $\begin{array}{l}\text { NA } \\
\text { NA }\end{array}$ & $\begin{array}{l}0 \\
0\end{array}$ & $\begin{array}{l}\text { NA } \\
\text { NA }\end{array}$ \\
\hline 1962 & 1,220 & NA & 54 & NA & 2,260 & NA & 100 & NA & 10,554 & NA & 467 & NA & 0 & NA & 0 & NA \\
\hline 1963 & 1,062 & NA & 47 & NA & 966 & NA & 87 & NA & 8,814 & NA & 390 & N & 0 & NA & 0 & NA \\
\hline 1964 & 1,017 & NA & 45 & NA & 1,898 & NA & 84 & NA & 7,729 & NA & 342 & $N$ & 0 & NA & 0 & NA \\
\hline 1965 & 1,153 & NA & 51 & NA & 2,147 & NA & 95 & NA & 7,707 & NA & 341 & & 0 & NA & 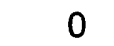 & NA \\
\hline 1966 & 1.062 & NA & 47 & NA & 1,966 & NA & 87 & NA & 7,119 & NA & 315 & & 0 & NA & 0 & NA \\
\hline 1967 & 746 & NA & 33 & NA & 1,379 & NA & 61 & $N$ & 5,447 & NA & 241 & & 0 & NA & 0 & NA \\
\hline 1968 & 542 & NA & 24 & NA & 994 & NA & 44 & NA & 4,836 & NA & 214 & $N$ & 0 & NA & 0 & NA \\
\hline 1969 & 520 & NA & 23 & NA & 994 & NA & 44 & NA & 5,017 & NA & 222 & $\mathrm{~N}$ & 0 & NA & 0 & NA \\
\hline 1970 & 271 & 0.95 & 12 & 21.47 & 520 & 0.52 & 23 & 11.75 & 4,746 & 0.52 & 210 & 11.75 & 0 & NA & 0 & NA \\
\hline 1971 & 384 & 0.99 & 17 & 22.33 & 701 & 0.60 & 31 & 13.47 & 5,040 & 0.60 & 223 & 13.4 & 0 & NA & 0 & NA \\
\hline 1972 & 249 & 1.03 & 11 & 23.19 & 475 & 0.67 & 21 & 15.1 & 3,277 & 0.67 & 145 & 15.19 & 1,231 & NA & 2,002 & NA \\
\hline 1973 & 136 & 1.06 & $\hat{3}$ & 24.05 & 271 & 0.75 & 12 & 16.90 & 3,729 & 0.75 & 165 & 16.90 & 58,360 & 0.39 & 3,741 & 6.08 \\
\hline 1974 & 113 & 1.10 & 5 & 24.91 & 203 & 0.82 & 9 & 18.6 & 6,192 & 0.82 & 274 & 18.62 & 5,630 & 0.37 & 2,925 & 5.77 \\
\hline 1975 & 158 & 1.14 & 7 & 25.76 & 294 & 0.90 & 13 & 20.3 & 10,464 & 0.90 & 463 & 20.34 & 62,540 & 0.56 & 4,009 & 8.74 \\
\hline 1976 & 181 & 1.76 & 8 & 39.87 & 339 & 1.18 & 15 & 26.58 & 15,029 & 1.18 & 665 & 26.58 & 64,069 & 0.75 & 4,107 & 11.70 \\
\hline 1977 & 1,062 & 2.39 & 47 & 53.97 & 1,966 & 1.45 & 87 & 32.82 & 12,724 & 1.45 & 563 & 32.82 & 83,772 & 0.72 & 5,370 & 11.23 \\
\hline 1978 & 1,446 & 3.01 & 64 & 68.07 & 2,667 & 1.73 & 118 & 39.05 & 12,611 & 1.73 & 558 & 39.05 & 66,035 & 0.77 & 4,233 & 12.01 \\
\hline 1979 & 1.040 & 3.64 & 46 & 82.17 & 1.921 & 2.00 & 85 & 45.29 & 12,746 & 2.00 & 564 & 45.29 & 80,590 & 0.84 & 5,166 & 13.10 \\
\hline 1980 & 1,311 & 4.26 & 58 & 96.28 & 2,441 & 2.28 & 108 & & 7,933 & 2.28 & 351 & 51.53 & 77,220 & 0.96 & 4,950 & 14.98 \\
\hline 1981 & 1,085 & 4.56 & 48 & 103.06 & 2,011 & 2.57 & 89 & 58.08 & 8,226 & 2.57 & 364 & 58.08 & 79,424 & 1.11 & 4,964 & 17.76 \\
\hline 198 & 1,469 & 4.81 & 65 & & 2,735 & 2.67 & 121 & & 8,678 & 2.67 & 384 & & 61,504 & 1.39 & 3,844 & 22.24 \\
\hline 1983 & 1,921 & 3.75 & 85 & 84.75 & 3,571 & 2.73 & 158 & 62.58 & 4,904 & 2.34 & 217 & 52.88 & 68,768 & 1.62 & 4,298 & 25.92 \\
\hline 198 & 1,808 & 3.76 & 80 & & & 2.87 & 149 & & 4,497 & 2.36 & 199 & & 72,096 & 1.72 & 4,506 & 27.52 \\
\hline 1985 & 1,709 & 3.46 & 76 & 78.20 & 3,173 & 2.86 & 140 & 67.89 & 4,701 & 2.17 & 208 & 49.13 & 83,072 & 1.69 & 5,192 & 27.07 \\
\hline 1986 & 704 & 3.11 & 31 & 70.29 & 1,307 & 2.81 & 58 & 67.40 & 4,475 & 1.96 & 198 & 44.30 & 53,280 & 1.74 & 3,330 & 27.84 \\
\hline
\end{tabular}

Source: Washington State Energy Office, "Washington State Energy Use Profile, 1960 -1986," 1988. 

Consumption and Price

\begin{tabular}{|c|c|c|c|c|c|c|c|c|}
\hline \multirow[b]{2}{*}{ Year } & \multicolumn{4}{|c|}{ Residential Heating Oil-_____ } & \multicolumn{4}{|c|}{-Residual Fuels- } \\
\hline & $\begin{array}{r}\text { Billion } \\
\text { Btu }\end{array}$ & $\begin{array}{l}\text { Dollars per } \\
\text { Million Btu }\end{array}$ & $\begin{array}{c}\text { Thousand } \\
\text { Barrels }\end{array}$ & $\begin{array}{l}\text { Dollars per } \\
\text { Gallon }\end{array}$ & $\begin{array}{r}\text { Billion } \\
\text { Btu }\end{array}$ & $\begin{array}{l}\text { Dollars per } \\
\text { Million Btu }\end{array}$ & $\begin{array}{r}\text { Thousand } \\
\text { Barrels }\end{array}$ & $\begin{array}{l}\text { Dollars per } \\
\text { Gallon }\end{array}$ \\
\hline 1960 & 42,540 & 1.08 & 7,303 & 0.150 & 58,475 & NA & 9,301 & NA \\
\hline 1961 & 41,427 & 1.12 & 7,112 & 0.156 & 47,574 & NA & 7,567 & NA \\
\hline 1962 & 38,567 & 1.12 & 6,621 & 0.156 & 47,869 & NA & 7,614 & NA \\
\hline 1963 & 38,317 & 1.15 & 6,578 & 0.160 & 48,209 & NA & 7,668 & NA \\
\hline 1964 & 41,282 & 1.16 & 7,087 & 0.161 & 55,162 & NA & 8,774 & NA \\
\hline 1965 & 37,833 & 1.15 & 6,495 & 0.160 & 57,457 & NA & 9,139 & NA \\
\hline 1966 & 39,767 & 1.18 & 6,827 & 0.164 & 62,166 & NA & 9,888 & NA \\
\hline 1967 & 34,880 & 1.22 & 5,988 & 0.169 & 60,971 & NA & 9,698 & NA \\
\hline 1968 & 38,952 & 1.25 & 6,687 & 0.174 & 75,142 & NA & 11,952 & NA \\
\hline 1969 & 39,645 & 1.28 & 6,806 & 0.178 & 70,823 & NA & 11,265 & NA \\
\hline 1970 & 40,979 & 1.33 & 7,035 & 0.185 & 65,284 & 0.39 & 10,384 & 0.058 \\
\hline 1971 & 40,565 & 1.41 & 6,964 & 0.196 & 59,607 & 0.53 & 9,481 & 0.079 \\
\hline 1972 & 38,783 & 1.42 & 6,658 & 0.197 & 74,337 & 0.57 & 11,824 & 0.085 \\
\hline 1973 & 36,453 & 1.64 & 6,258 & 0.228 & 71,269 & 0.70 & 11,336 & 0.105 \\
\hline 1974 & 28,484 & 2.60 & 4,890 & 0.360 & 64,027 & 1.49 & 10,184 & 0.223 \\
\hline 1975 & 27,995 & 2.72 & 4,806 & 0.377 & 53,207 & 1.70 & 8,463 & 0.254 \\
\hline 1976 & 28,909 & 2.93 & 4,963 & c 106 & 46,612 & 1.60 & 7,414 & 0.240 \\
\hline 1977 & 31,898 & 3.32 & 5,476 & 0.460 & 60,582 & 1.72 & 9,636 & 0.257 \\
\hline 1978 & 31,193 & 3.50 & 5,355 & 0.486 & 72,099 & 1.67 & 11,468 & 0.250 \\
\hline 1979 & 28,210 & 5.03 & 4,843 & 0.697 & 81,071 & 2.19 & 12,895 & 0.328 \\
\hline 1980 & 19.933 & 7.27 & 3,422 & 1.008 & 108,815 & 3.24 & 17,308 & 0.485 \\
\hline 1981 & 17,475 & 8.40 & 3,000 & 1.165 & 102,975 & 5.03 & 16,379 & 0.753 \\
\hline 1982 & 18,914 & 8.48 & 3,247 & 1.176 & 85,151 & 5.19 & 13,544 & 0.777 \\
\hline 1983 & 15,687 & 7.86 & 2,693 & 1.090 & 31,184 & 5.12 & 4,960 & 0.766 \\
\hline 1984 & 17,731 & 7.40 & 3,044 & 1.026 & 32,202 & 5.26 & 5,122 & 0.787 \\
\hline 1985 & 18,028 & 7.29 & 3,095 & 1.011 & 52,251 & 3.80 & 8,311 & 0.569 \\
\hline 1986 & 18,930 & 5.26 & 3,250 & 0.730 & 54,877 & 1.93 & 8,729 & 0.289 \\
\hline
\end{tabular}

Source: Washington State Energy Office, Washington State Energy Use Profile, 1960 -1986, 1988 


\section{Part V}

\section{Bibliography}




\section{Part V}

\section{Bibliography}

Energy Information Administration. Electric Power Monthly. March 1991.

Energy Information Administration. Natural Gas Monthly. January 1991.

Energy Information Administration. Quarterly Coal Report. December 1989.

Howard, J.O. Ratios for Estimating Logging Residues in the Pacific Northwest. 1981.

Howard, J.O. and F.R. Ward. Oregon's Forest Products Industry1985. 1988.

Kerstetter, James D. Industrial Wood Fuel Market Assessment in Washington State. 1986.

Larson, D.N. and D.A. Bearden. 1984 Mill Survey. 1986.

Miller Freeman Publications. 1988 Directory of the Forest Products Industry. 1988.

Northwest Public Power Association. 1990 Northwest Electric Utility Directory. 1991.

Office of Financial Management. State of Washington Data Book. 1987.

Office of Financial Management. State of Washington Data Book. 1989.

Resource Information System, Inc. Supply, Demand, and Prices of Pacific Northwest Timber, Volume I: An Assessment of Market Opportunities in an Environment of Increasing Competition. 1988.

Taylor, Roy. Agricultural Residues. July 1987.

The Chilton Company. Energy User News. January 1991.

The Matrix Management Group, R.W. Beck and Associates, and the Gilmore Research Group. Best Management Practices Analysis for Solid Waste, 1987 Recycling and Waste Stream Survey.

Volume 1. Prepared for the Office of Waste Reduction and Recycling, Washington State Department of Ecology. 1988. 
U.S. Department of Energy and the Bonneville Power Administration. Biomass Energy Project Development Guidebook. Prepared for the Pacific Northwest and Alaska Bioenergy Program. 1989.

U.S. Department of Energy and the Bonneville Power Administration. Regional Logging Residue Curve Project. Volume 1, Final Report. August 1986.

U.S. Department of Commerce. 1982 Census of Agriculture, Washington State and County Data. U.S. Government Printing Office, Washington, D.C. May 1984.

U.S. Department of Commerce. County Business Patterns 1986, Washington. U.S. Government Printing Office, Washington, D.C. 1988.

Washington State Department of Agriculture. Washington Agricultural Statistics 1982-83. December 1983.

Washington State Department of Agriculture. Washington Agricultural Statistics 1983-84. December 1984.

Washington State Department of Agriculture. Washington Agricultural Statistics 1985-86. September 1986.

Washington State Department of Agriculture. Washington Agricultural Statistics 1986-1987. November 1987.

Washington State Department of Agriculture. Washington Agricultural Statistics 1987-1988. November 1988.

Washington State Department of Agriculture. Washington Agricultural Statistics 1988-89. October 1989.

Washington State Department of Health. Directory of Licensed Hospitals. 1989.

Washington State Department of Natural Resources. 1978 Washington Mill Survey. 1979.

Washington State Department of Natural Resources. 1980 Washington Mill Survey. 1982.

Washington State Department of Natural Resources. 1982 Washington Mill Survey. 1984.

Washington State Department of Natural Resources. 1984 Washington Mill Survey. 1986.

Washirgton State Department of Natural Resources. Washington Mil! Survey -1986. 1990.

Washington State Department of Natural Resources. 1988

Washington Mill Survey. Preliminary data. 1991. 
Washington State Department of Natural Resources. 1980 Timber Harvest Report. 1982.

Washington State Department of Natural Resources. 1981 Timber Harvest Report. 1983.

Washington State Department of Natural Resources. 1982 Timber Harvest Report. 1983.

Washington State Department of Natural Resources. 1983 Timber Harvest Report. 1983.

Washington State Department of Natural Resources. 1984 Timber Harvest Report. 1985.

Washington State Department of Natural Resources. 1985 Timber Harvest Report. 1986.

Washington State Department of Natural Resources. 1986 Timber Harvest Report. 1988.

Washington State Department of Natural Resources. 1987 Timber Harvest Report. 1989.

Washington State Department of Natural Resources. 1988 Timber Harvest Report. 1989.

Washington State Energy Office. Data collected by the Energy Partnership program. 1991.

Washington State Energy Office. 1990 Washington State Directory of Biomass Energy Facilities. 1990.

Washington State Energy Office. Washington State Energy Use Profile 1960 - 1986. January 1988.

Washington State Energy Office. Washington State Petroleum Markets Data Book. December 1989.

Washington State Energy Office. 1990 Wood Residue Survey and Directory of Secondary Wood Processing Facilities in Washington State. 1990.

Yates, Richard and Charity. Washington State Atlas, A Political \& Economic View of the Evergreen State. The Information Press. 1985.

Yates, Richard and Charity. 1986 Washington State Yearbook. The Information Press. 1986.

Yates, Richard and Charity. 1989 Washington State Yearbook. The Information Press. 1989. 
Appendix A

Glossary 


\section{Appendix A}

\section{Glossary}

Agricultural residues: Above-ground organic matter left in the field after the harvest of a crop (in this publication it is stubble and straw left from harvesting barley and wheat).

Gross agricultural residues: All residues generated from agricultural activities.

Net agricultural residues: Agricultural residues available for energy consumption.

Bioenergy: The conversion of the complex carbohydrates in organic matter into energy, either by using it directly as a fuel or by processing it into liquids and gases that are more efficient.

Biogas: A combustible gas derived from decomposing biological waste.

Biomass: Any organic matter that is available to be used as a fuel on a renewable basis including forest residues, agricultural crops and wastes, wood and wood wastes, animal wastes, livestock operation residue, aquatic plants, and municipal wastes.

Biomass fuel: Any liquid, solid, or gaseous fuel produced by conversion from biomass.

Bone dry (oven dry): Having zero percent moisture content.

Wood heated in an oven at a constant temperature of 212 degrees $F$. or above until its weight stabilizes.

British Thermal Unit (Btu): The amount of heat required to raise the temperature of 1 pound of water 1 degree Fahrenheit.

Capacity: The maximum instantaneous output of a resource, often expressed in kilowatts or megawatts.

Cogeneration: Technology to produce electric energy and forms of useful energy (thermal or mechanical) for industrial, commercial, heating, or cooling purposes, through the sequential use of an energy source.

Combustion: A chemical process, especially oxidation, accompanied by the production of heat and light.

Cubic foot (CF): $12^{\prime \prime} \times 12^{\prime \prime} \times 12^{\prime \prime}$ or $1^{\prime} \times 1^{\prime} \times 1^{\prime}$

Dr̃y tôñ: 2,000 loss. of materiai diried to a consiant weighit. 
Feedstock: Any material that is converted to another form or product.

Forest residues: Wasted and unused wood in the forest, including logging residue; rough, rotten, and dead trees; and annual mortality.

Fuel handling system: The delivered wood fuel is unloaded from vans or trucks and transported to a storage pile or bin. From storage, the wood fuel is conveyed mechanically or pneumatically to the energy conversion system.

Hogged (hog) fuel: Wood waste such as sawdust, planer shavings, and wood chunks normally used for fuel purposes.

Kilowatt: 1,000 Watts, a measure of electrical power.

Logging residues: The unused portions of saw timber and pole timber trees cut or killed during the harvesting process.

Megawatt (MW): An electrical generation unit of one million Watts or $1,000 \mathrm{~kW}$.

Mill residue: Wood and bark residues produced in processing lumber.

Municipal solid waste (MSW): Waste generated from the commercial, industrial, residential, and government sectors of our economy.

Private ownerships: Include forest industry and large and small nonindustrial companies and individuals.

Forest industry: Companies and individuals operating wood-using plants with no acreage limitations.

Large nonindustrial: Companies and individuals not operating wood-using plants and having holdings of 1,000 or more acres.

Small nonindustrial: Companies and individuals not operating wood-using plants and having holdings of less than 1,000 acres.

Public ownership lands: Owned by U.S. Forest Service, State, Bureau of Indian Affairs, and other Federal and Nonfederal lands.

State: Harvest from Department of Natural Resourcesmanaged trust lands.

Other Nonfederal: Public land owned by cities, counties, public utility districts, and state agencies other than the Department of Natural Resources.

Forest Service: National forest harvest. 
Bureau of Indian Affairs (BIA) - Timber harvest from several Native American tribes (Colville, Quinault, Yakima, etc.) located in Washington state.

Other Federal - Other federal agencies (military, National Park Service, Wildlife Service) selling timber for industrial wood harvest in the state.

RDF: Refuse-derived fuel: the combustible portion of solid waste that has been processed to remove heavier, noncombustible materials.

Renewable energy resource: Any energy resource that has recently originated in the sun, including direct and indirect solar radiation and intermediate solar energy forms such as wind, ocean thermal gradients, ocean currents and waves, hydropower, photovoltaic energy products of photosynthetic processes, organic wastes, and others.

Secondary wood processing facility: Companies that use lumber to produce their products (manufacturers of flooring, millwork, kitchen cabinets, boxes, pallets, skids, mobile homes, and prefabricated wood buildings).

Watt: The common base unit of power in the metric system. 1 kilowatt $=1,000$ watts; $1 \mathrm{kWh}$ (kilowatt hour) $=$ the amount of power consumed for one hour. 1 megawatt $=1,000,000$ watts.

Note: A number of these definitions were excerpted from U.S. Department of Energy, Pacific Northwest and Alaska Bioenergy Program Glossary, (1985), the U.S. Department of Energy Biomass Energy Project Development Guidebook, 1989, and Washington State Department of Natural Resources, Washington Timber Harvest-1988. 
Appendix B

Spokane County Profile 


\section{SPOKANE}

County Seat

West 1116 Broadway

Spokane, Washington 99260

Phone: (509) 456-2265

\section{GENERAL COUNTY INFORMATION1,2,3,4}

Area (Square Miles):

Assessed Value:

Per Capita Personal Income:

Publlc Lands Acreages:

Total Land Acreage

Public Lands

Federal Lands

State Lands

Indian Lands

County Lands

\author{
1,764 \\ $\$ 9,417,000,000$ \\ 13,656
}

$1,125,120$

100,458

21,396

51,371

27,691
Rank: $\quad 19$

Rank: $\quad 4$

Rank: $\quad 19$

\section{Temperature:}

Average Annual Maximum Temperature:

Average Annual Minimum Temperature:

Average Annual Precipitation:

17.41 inches

Population:

\begin{tabular}{rrrrrrrrr}
\hline Yr. $\quad 0-9$ & $10-19$ & $20-29$ & $30-39$ & $40-49$ & $50-59$ & $60-69$ & $70 \&$ Over & Total \\
\hline 8755,495 & 49,813 & 58,657 & 58,071 & 41,995 & 29,821 & 30,136 & 31,912 & 355,900 \\
8956,003 & 48,811 & 56,250 & 58,736 & 46,216 & 29,791 & 29,393 & 32,801 & 358,000
\end{tabular}

Major Cltles:

\begin{tabular}{lr}
\hline City & Population \\
\hline Spokane & 170,700 \\
Cheney & 7,630 \\
Medical Lake & 3,790 \\
Deer Park & 2,380 \\
Airway Heights & 1,905
\end{tabular}


Princlpal Economlc Actlvities:

Food Processing, Apparel \& Textile Manufacturing, Publishing, Metal Fabrication, Agriculture, Electronics, Machinery Manufacturing, and Wood Products

Major Industrles:

Services

Average No. of Employees:

34,858

502,589

Percent of Total:

25.6

Wages Paid ( $\$$ in 000's):

Percent of Total:

20.5

\begin{tabular}{lcccccccccc}
\hline Employees: & $1-4$ & $5-9$ & $10-19$ & $20-49$ & $50-99$ & $100-249$ & $250-499$ & $500-999$ & $>999$ \\
\hline 1986 Firms: & 1,800 & 622 & 308 & 180 & 64 & 27 & 7 & 5 & 3 \\
1988 Firms: & 1,713 & 666 & 376 & 192 & 64 & 32 & 8 & 4 & 3
\end{tabular}

Retall Trade

Average No. of Employees:

28,465

Percent of Total: $\quad 20.9$

Wages Paid ( $\$$ in 000 's):

302,967

Percent of Total: $\quad 12.4$

\begin{tabular}{lccccccccc}
\hline Employees: & $1-4$ & $5-9$ & $10-19$ & $20-49$ & $50-99$ & $100-249$ & $250-499$ & $500-999$ & $>999$ \\
\hline 1986 Firms: & 930 & 530 & 305 & 264 & 78 & 20 & 2 & 1 & 0 \\
1988 Firms: & 843 & 534 & 305 & 265 & 88 & 24 & 1 & 0 & 0
\end{tabular}

Government (Local, State, Federal)

Average No. of Employees:

Wages Paid ( $\$$ in 000 's):

22,812

517,545
Percent of Total: $\quad 16.8$

Percent of Total: 21.1

Manufacturing

Average No. of Employees:

18,770

442,098

Percent of Total: $\quad 13.8$

Percent of Total: 18

\begin{tabular}{lcccccccccc}
\hline Employe日s: & $1-4$ & $5-9$ & $10-19$ & $20-49$ & $50-99$ & $100-249$ & $250-499$ & $500-999$ & $>999$ \\
\hline 1986 Firms: & 173 & 87 & 67 & 70 & 36 & 20 & 3 & 2 & 4 \\
1988 Firms: & 160 & 103 & 79 & 70 & 37 & 24 & 2 & 8 & 2 & \\
& & & & & & & & & &
\end{tabular}

Wholesale Trade

Average No. of Employees:10,091 Percent of Total: 7.4

Wages Paid (\$ in 000's):223,271 Percent of Total: $\quad 19.1$

\begin{tabular}{lccccccccc}
\hline Employees: & $1-4$ & $5-9$ & $10-19$ & $20-49$ & $50-99$ & $100-249$ & $250-499$ & $500-999$ & $>999$ \\
\hline 1986 Firms: & 307 & 199 & 181 & 88 & 25 & 5 & 3 & 0 & 0 \\
1988 Firms: & 315 & 220 & 172 & 79 & 19 & 7 & 2 & 0 & 0
\end{tabular}


Economic Development Assistance:

Spokane Area EDC

Phone: (509) 624-9285

P.O. Box 203 (N. 221 Wall. Suite 310)

Spokane, Washington 99201

Infrastructure:

Highways: Interstate 90; State 27, 206, 290, 291, 902, 904; U.S 2, 195, 395

Railroads: Burlington Northem, Union Pacific

Airports: Spokane, Deer Park, Felts Field, Spokane International

Mineral Resources, Energy Potentlal:

Type: Uranium

Power Generating Facllities:

Long Lake

Type: Hydroelectric

MW: 70

\section{Electric Utilities:}

City of Cheney Light Department Municipality Operating with BPA

609 Second Street

Cheney, WA 99004

(509) 235-7211

Year: 1987

KWh Sales: $93,745,374$

Dollar Sales: $\$ 3,685,796$

Average Cost per kwh: $\$ 0.04$

iritind Power \& Light Company

Cooperative Operating with BPA

P.O. Box 4429

East 320 Second Avenue

Spokane, WA 99202

(509) $747-7151$

Year: 1989

KWh Sales: $433,866,000$

Dollar Sales: $\$ 20,912,000$

Average Cost per kwt: $\$ 0.05$
Kootenai Electric Cooperative Cooperative Operating with BPA

P.O. Box 278

Hayden Lake, ID

(208) 765-1200

Year:

KWh Sales:

Dollars Sales:

Average Cost per kwh:

Vera Water and Power

Cooperative Gperatiing without BPA

P.O. Box 630

601 North Evergreen

Veradale. WA 99037-0630

(509) 924-3800

Year: 1989

KWh Sales: $162,781,390$

Dollar Sales: $\$ 5,587,270$

Average Cost per kwh: $\$ 0.03$ 
Modern Electic Company

Cooperative Operating without BPA

Box 14008, Opportunity Branch North

904 Pines Road (Opportunity)

Spokane, WA 99214

(509) $928-4540$

Year: 1988

KWh Sales: $165,000,000$

Dollar Sales: $\$ 6,779,000$

Average Cost per kwh: $\$ 0.03$
The Washington Water Power Company Investor Owned Utility

Ms. Dorothy Mercer, Rate \& Research

P.O. Box 3727

Spokane, WA 99220

(509) 489-0500

Year: 1988

KWh Sales: $\$, 306,000,000$

Dollar Sales: $\$ 371,000,000$

Average Cost per kwh: $\$ 0.04$

\section{AGRICULTURE 5}

Agricultural Extension Contacts:

Spokane County

N. 222 Havana

Spokane, WA 99202

(509) 456-3651

Faculty:

Jonathan R. Newkirk, Chair, ANR

E. Adams, ANR, CRD Area: Adams, Lincoln, Ped Oreille, Steven

Tonie J. Fitzgerald, ANR Area: Stevens, Pend Oreille

Dobra S. Marple, 4-H

Program Staft:

Janet Edwards, 4-H Ext. Program Assistant

Clarice R. Reilly, Research Ext. Prog. Assistant

Sydney McCrea, Master Gardener Program Coordinator

\section{Farms and Cropland:}

I tarvested Cropland-Farms:

Harvested Cropland-Acres:

Cropland for Grazing-Farm:

Cropland for Grazing-Acre:

Other Cropland-Farms:

Other Cropland-Acres: 
Spokane County Annual Spring Wheat Residues (Dryland)

\begin{tabular}{|c|c|c|c|c|c|c|c|c|}
\hline Year & $\begin{array}{c}\text { Acreage } \\
\text { Harvested }\end{array}$ & $\begin{array}{l}\text { Gross } \\
\text { Tons }\end{array}$ & $\begin{array}{l}\text { Straw Net } \\
\text { Tons }\end{array}$ & $\begin{array}{l}\text { Straw Net } \\
\text { Tons/Acre }\end{array}$ & $\begin{array}{l}\text { Straw } \\
\text { MBTU }\end{array}$ & $\begin{array}{l}\text { Net Bushels } \\
\text { per Acre }\end{array}$ & $\begin{array}{l}\text { Net MBTU } \\
\text { per Acre }\end{array}$ & $\begin{array}{c}\text { Harvested } \\
\text { Bushels }\end{array}$ \\
\hline 1982 & 10,500 & 17,199 & 0 & 0 & 0 & 42 & 0 & 441,000 \\
\hline 1983 & 7,800 & 11,232 & 0 & 0 & 0 & 37 & 0 & 288,000 \\
\hline 1984 & 6,500 & 11,115 & 0 & 0 & 0 & 44 & 0 & 285,000 \\
\hline 1985 & 9,400 & 10,452 & 0 & 0 & 0 & 29 & 0 & 268,000 \\
\hline 1986 & 8,400 & 10,608 & 0 & 0 & 0 & 32 & 0 & 272,000 \\
\hline 1987 & 6,200 & 10,257 & 0 & 0 & 0 & 42 & 0 & 263,000 \\
\hline 1988 & 6,400 & 11,895 & 0 & 0 & 0 & 48 & 0 & 305,000 \\
\hline 1989 & 42,800 & 76,752 & 0 & 0 & 0 & 46 & 0 & $1,968,000$ \\
\hline Min.: & 6,200 & 10,257 & 0 & 0 & 0 & 29 & 0 & 263,000 \\
\hline Max.: & 42,800 & 76,752 & 0 & 0 & 0 & 48 & 0 & $1,968,000$ \\
\hline Ave.: & 12,250 & 19,939 & 0 & 0 & 0 & 40 & 0 & 511,250 \\
\hline
\end{tabular}

Spokane County Annual Spring Wheat Residues (Irrigated Land)

\begin{tabular}{|c|c|c|c|c|c|c|c|c|}
\hline Year & $\begin{array}{c}\text { Acreage } \\
\text { Harvested }\end{array}$ & $\begin{array}{l}\text { Gross } \\
\text { Tons }\end{array}$ & $\begin{array}{c}\text { Straw Net } \\
\text { Tons }\end{array}$ & $\begin{array}{l}\text { Straw Net } \\
\text { Tons/Acre }\end{array}$ & $\begin{array}{l}\text { Straw } \\
\text { MBTU }\end{array}$ & $\begin{array}{l}\text { Net Bushels } \\
\text { per Acre }\end{array}$ & $\begin{array}{l}\text { Net MBTU } \\
\text { per Acre }\end{array}$ & $\begin{array}{c}\text { Harvested } \\
\text { Bushels }\end{array}$ \\
\hline 1982 & 3,000 & 8,190 & 2,190 & 1 & 32,850 & 70 & 11 & 210,000 \\
\hline 1983 & 2,800 & 8,190 & 2,590 & 1 & 38,850 & 75 & 14 & 210,000 \\
\hline 1984 & 1,700 & 5,460 & 2,060 & 1 & 30,900 & 82 & 18 & 140,000 \\
\hline 1985 & 600 & 1,521 & 321 & 1 & 4,815 & 65 & 8 & 39,000 \\
\hline 1986 & 600 & 1,248 & 48 & 0 & 720 & 53 & 1 & 32,000 \\
\hline 1987 & 400 & 1,404 & 604 & 2 & 9,060 & 90 & 23 & 36,000 \\
\hline 1988 & 700 & 1,404 & 4 & 0 & 60 & 51 & 0 & 36,000 \\
\hline 1989 & 2,200 & 6,552 & 2,152 & 1 & 32,280 & 76 & 15 & 168,000 \\
\hline Min.: & 400 & 1,248 & 4 & 0 & 60 & 51 & 0 & 32,000 \\
\hline Max.: & 3,000 & 8,190 & 2,590 & 2 & 38,850 & 90 & 23 & 210,000 \\
\hline Ave.: & 1,500 & 4,246 & 1,246 & 1 & 18,692 & 70 & 11 & 108,875 \\
\hline
\end{tabular}

Spokane County Annual Winter Wheat Residues (Dryland)

\begin{tabular}{|c|c|c|c|c|c|c|c|c|}
\hline Year & $\begin{array}{c}\text { Acreage } \\
\text { Harvested }\end{array}$ & $\begin{array}{l}\text { Gross } \\
\text { Tons }\end{array}$ & $\begin{array}{l}\text { Straw Net } \\
\text { Tons }\end{array}$ & $\begin{array}{l}\text { Straw Net } \\
\text { Tons/Acre }\end{array}$ & $\begin{array}{l}\text { Straw } \\
\text { MBTU }\end{array}$ & $\begin{array}{c}\text { Net Bushels } \\
\text { per Acre }\end{array}$ & $\begin{array}{l}\text { Net MBTU } \\
\text { per Acre }\end{array}$ & $\begin{array}{c}\text { Harvested } \\
\text { Bushels }\end{array}$ \\
\hline 1982 & 121,700 & 345,219 & 40,969 & 0 & 614,535 & 56 & 5 & $6,769,000$ \\
\hline 1983 & 118,700 & 442,476 & 145,726 & 1 & $2,185,890$ & 73 & 18 & $8,676,000$ \\
\hline 1984 & 123,300 & 344,964 & 36,714 & 0 & 550.710 & 55 & 5 & 6.764 .000 \\
\hline 1985 & 102,300 & 231,540 & 0 & 0 & 0 & 44 & 0 & $4,540,000$ \\
\hline 1986 & 98,300 & 275,247 & 29,497 & 0 & 442.455 & 55 & 5 & $5,397,000$ \\
\hline 1987 & 89,900 & 313,854 & 89,104 & 1 & $1,336.560$ & 69 & 15 & $6,154,000$ \\
\hline 1988 & 81.000 & 321,504 & 119,004 & 2 & 1.785 .060 & 78 & 22 & $6.304,000$ \\
\hline 1989 & 79,900 & 230.622 & 30,872 & 0 & 463.080 & $5 i$ & 6 & $4,522,000$ \\
\hline Muiñ.: & 79,900 & 230,622 & 0 & 0 & 0 & 44 & o & $4,522,000$ \\
\hline Max.: & 123,300 & 442,476 & 145,726 & 1 & $2,185,890$ & 78 & 22 & $8,676,000$ \\
\hline Ave.: & 101,888 & 313,178 & 61.486 & 1 & 922,286 & 61 & 9 & $6,140,750$ \\
\hline
\end{tabular}


Spokane County Annual Winter Wheat Residues (Irrigated Land)

\begin{tabular}{|c|c|c|c|c|c|c|c|c|}
\hline Year & $\begin{array}{c}\text { Acreage } \\
\text { Harvested }\end{array}$ & $\begin{array}{l}\text { Gross } \\
\text { Tons }\end{array}$ & $\begin{array}{l}\text { Straw Net } \\
\text { Tons }\end{array}$ & $\begin{array}{l}\text { Straw Net } \\
\text { Tons/Acre }\end{array}$ & $\begin{array}{l}\text { Straw } \\
\text { MBTU }\end{array}$ & $\begin{array}{l}\text { Net Bushels } \\
\text { per Acre }\end{array}$ & $\begin{array}{l}\text { Net MBTU } \\
\text { per Acre }\end{array}$ & $\begin{array}{c}\text { Harvested } \\
\text { Bushels }\end{array}$ \\
\hline 1982 & 6,000 & 27,693 & 15,693 & 3 & 235,395 & 91 & 39 & 543,000 \\
\hline 1983 & 7,200 & 32,079 & 17,679 & 3 & 265,185 & 87 & 37 & 629,000 \\
\hline 1984 & 7,300 & 33,048 & 18,448 & 3 & 276,720 & 89 & 38 & 648,000 \\
\hline 1985 & 800 & 3,009 & 1,409 & 2 & 21,135 & 74 & 26 & 59,000 \\
\hline 1986 & 1,000 & 4,080 & 2,080 & 2 & 31,200 & 80 & 31 & 80,000 \\
\hline 1987 & 1,000 & 5,100 & 3,100 & 3 & 46,500 & 100 & 47 & 100,000 \\
\hline 1988 & 1,500 & 6,885 & 3,885 & 3 & 58,275 & 90 & 39 & 135,000 \\
\hline 1989 & 1,100 & 5,049 & 2,849 & 3 & 42,735 & 90 & 39 & 99,000 \\
\hline Min. & 800 & 3,009 & 1,409 & 2 & 21,135 & 74 & 26 & 59,000 \\
\hline Max. & 7,300 & 33,048 & 18,448 & 3 & 276,720 & 100 & 47 & 648,000 \\
\hline Ave. & 3,238 & 14,618 & 8,143 & 2 & 122,143 & 88 & 37 & 286,625 \\
\hline
\end{tabular}

Spokane County Annual Barley Residues (Dryland)

\begin{tabular}{|c|c|c|c|c|c|c|c|c|}
\hline Year & $\begin{array}{c}\text { Acreage } \\
\text { Harvested }\end{array}$ & $\begin{array}{l}\text { Gross } \\
\text { Tons }\end{array}$ & $\begin{array}{c}\text { Straw Net } \\
\text { Tons }\end{array}$ & $\begin{array}{l}\text { Straw Net } \\
\text { Tons/Acre }\end{array}$ & $\begin{array}{l}\text { Straw } \\
\text { MBTU }\end{array}$ & $\begin{array}{l}\text { Net Bushels } \\
\text { per Acre }\end{array}$ & $\begin{array}{l}\text { Net MBTU } \\
\text { per Acre }\end{array}$ & $\begin{array}{c}\text { Harvested } \\
\text { Bushels }\end{array}$ \\
\hline 1982 & 89,600 & 137,088 & 0 & 0 & 0 & 51 & 0 & $4,569,600$ \\
\hline 1983 & 75,400 & 158,340 & 0 & 0 & 0 & 70 & 0 & $5,278,000$ \\
\hline 1984 & 86,200 & 145,320 & 0 & 0 & 0 & 56 & 0 & $4,844,000$ \\
\hline 1985 & 66,500 & 87,570 & 0 & 0 & 0 & 44 & 0 & $2,919,000$ \\
\hline 1986 & 68,700 & 105,150 & 0 & 0 & 0 & 51 & 0 & $3,505,000$ \\
\hline 1987 & 60,200 & 105,780 & 0 & 0 & 0 & 59 & 0 & $3,526,000$ \\
\hline 1988 & 48,500 & 97,470 & 0 & 0 & 0 & 67 & 0 & $3,249,000$ \\
\hline 1989 & 51,000 & 94,884 & 0 & 0 & 0 & 62 & 0 & $3,162,800$ \\
\hline Min. & 48,500 & 87,570 & 0 & 0 & 0 & 44 & 0 & $2,919,000$ \\
\hline Max. & 89,600 & 158,340 & 0 & 0 & 0 & 70 & 0 & $5,278,000$ \\
\hline Ave. & 68,263 & 116,450 & 0 & 0 & 0 & 57 & 0 & $3,881,675$ \\
\hline
\end{tabular}

Spokane County Annual Barley Residues (Irrigated Land)

\begin{tabular}{|c|c|c|c|c|c|c|c|c|}
\hline Year & $\begin{array}{c}\text { Acreage } \\
\text { Harvested }\end{array}$ & $\begin{array}{l}\text { Gross } \\
\text { Tons }\end{array}$ & $\begin{array}{c}\text { Straw Net } \\
\text { Tons }\end{array}$ & $\begin{array}{l}\text { Straw Net } \\
\text { Tons/Acre }\end{array}$ & $\begin{array}{l}\text { Straw } \\
\text { MBTU }\end{array}$ & $\begin{array}{c}\text { Net Bushels } \\
\text { per Acre }\end{array}$ & $\begin{array}{l}\text { Net MBTU } \\
\text { per Acre }\end{array}$ & $\begin{array}{c}\text { Harvested } \\
\text { Bushels }\end{array}$ \\
\hline 1982 & 3,400 & 8,976 & 2,176 & 1 & 32,640 & 88 & 10 & 299,200 \\
\hline 1983 & 3,200 & 9,984 & 3,584 & 1 & 53,760 & 104 & 17 & 332,800 \\
\hline 1984 & 3,800 & 10,800 & 3,200 & 1 & 48.000 & 95 & 13 & 360,000 \\
\hline 1985 & 2,500 & 5,430 & 430 & 0 & 6.450 & 72 & 3 & 181,000 \\
\hline 1986 & 800 & 1,740 & 140 & 0 & 2,100 & 73 & 3 & 58,000 \\
\hline 1987 & 500 & 1,650 & 650 & 1 & 9.750 & 110 & 20 & 55,000 \\
\hline 1988 & 1,200 & 3,960 & 1,560 & 1 & 23,400 & 110 & 20 & 132,000 \\
\hline 1989 & 1,100 & 3,597 & 1,397 & 1 & 20,955 & 109 & 19 & 119,900 \\
\hline Min.: & 500 & 1,650 & 140 & 0 & 2,100 & 72 & 3 & 55,000 \\
\hline Max.: & 3,800 & 10,800 & 3,584 & 1 & 53,760 & 110 & 20 & 360,000 \\
\hline Ave.: & 2,063 & 5,767 & 1,642 & 1 & 24,632 & 95 & 13 & $192, \tilde{2} 38$ \\
\hline & & & & B-6 & & & & \\
\hline
\end{tabular}




\section{BIOGAS}

Spokane Wastewater Treatment Plant

Spokane, WA

Capacity mgd: 44

Output mcubtt/yr: 20

Gross energy mbtu/yr: 120,000

\section{MUNICIPAL SOLID WASTE}

1989 Waste Generation:

1989 Population:

358,000

Estimated Lbs./Person:

6.26

Est. Annual Lbs./Person:

2,285

Estimated Total Waste Generated in 1989 (tons):

408,997

Spokane County Landfills: 6

\begin{tabular}{lll}
\hline Type & Facility Name & Owner \\
\hline Inert/Demolition & 44th \& Sands Landfill & \\
InertDemolition & 8th \& Camahan Pit & Joe Bascetta \\
Iner/Demolition & Bascetta Demo Site & Acme Concrete \\
InertDemolition & Broadway Pid & \\
Inert/Demolition & Central Pre-Mix Site & Acme Concrete \\
InertDemolition & KACC-Mead Landfill & Central Pre-Mix Company \\
Inert/Demolition & Old Materne Pit Site & Kaiser Aluminum \\
Inert/Demolition & Park Drive Landfill & Charles Materne \\
InertDemolition & Swan Disposal Site & Spokane County \\
Inert/Demolition & WS-C-12 Landfill & Robert Swan \\
Inert/Demolition & Whitman College Site & WA Dept. of Trans. \\
Inert/Demolition & Wilson Landfill & Whitman College \\
Inert/Demolition & Yardley Landfill & Ralph Wilson \\
Municipal & Marshall Landfill & Central Pre-Mix Company \\
Municipal & Mica Landfill & Glen Gillson \\
Municipal & Myers Landfill & Spokane County \\
Municipal & North Landfill & Thomas Myers \\
Municipal & South Landfill & Spokane County \\
Tire Pile & A.A. Auto Wrecking & City of Spokane \\
Tire Pile & Four Lake Tire Pile & A.A. Auto Wrecking \\
Tire Pile & Lindsay Tire Pile & AA Distributing \\
Tire Pile & Lindsey Tire Pile & John Lindsey \\
Tire Pile & Maak Tire Recyclers & John Lindsey \\
Transfer Station & Appleway T.S. & Carl Maak \\
Transfer Station & Deep Creek T.S. & \\
Transter Station & Fairtield T.S. & Spokane County \\
Transfer Station & Sunshine Recyclers T.S. & Spokane County \\
Woodwaste & Quartz Quarry Landiiii & Sunshine Disposal \\
& & Dan Loshbaugh \\
& &
\end{tabular}


Garbage Haulers:

Ada-Lin Waste Systems, Inc. North 2405 University Road Spokane, WA 99206

Western Refuse Company 1805 4th St.

Cheney, WA 99004

Hi-Valley Disposal, Inc.

E. 4004 Peone

P.O. Box 369

Mead, WA 99021
Metropolitan Services Corp. East 4004 Peone Road P.O. Box 369 Mead, WA 99021

Valley Garbage Service, Inc. East 11720 First Avenue P.O. Box 14000

Opportunity, WA 99206

Waste Paper Services, Inc. East 3407 Main Avenue Spokane, WA 99202

\section{WOOD RESIDUES}

Spokane County Annual Forest Residue -- All Land Ownerships 7

\begin{tabular}{lc}
\hline Year & Dry Tons \\
\hline 1981 & \\
1982 & 18,757 \\
1983 & 13,368 \\
1984 & 29,296 \\
1985 & 23,050 \\
1986 & 30,262 \\
1987 & 29,413 \\
1988 & 30,732 \\
& 28,306 \\
Minimum: & 13,367 \\
Maximum: & 30,732 \\
Average: & 25,398
\end{tabular}

Spokane County Annual Forest Residues on Private Lands ${ }^{7}$ (in Dry Tons)

\begin{tabular}{lrrrrl}
\hline Year & Industry & Large & Small & Total \\
\hline & & & & & \\
1981 & 991 & 765 & 15,050 & 16,806 & 12,302 \\
1982 & 441 & 955 & 10,906 & 26.426 \\
1983 & 0 & 2,486 & 23,941 & 22,918 & 28,821 \\
1984 & 645 & 5,539 & 16,735 & 27,888 & \\
1985 & 3,892 & 5,479 & 19,450 & 26,080 & \\
1986 & 3,631 & 5,039 & 19,218 & 24,158 & \\
1987 & 2,969 & 6,335 & 16,776 & 12,302 & \\
1988 & 54 & 5,935 & 18,170 & 28,821 & \\
& & 765 & & 10,906 &
\end{tabular}


Spokane County Annual Forest Residues on Public Lands 7 (in Dry Tons)

\begin{tabular}{lrrrrr}
\hline & National & State & Other & Bia & Total \\
\hline Year & 0 & 1,615 & 336 & 0 & 1,951 \\
1981 & 342 & 286 & 438 & 0 & 1066 \\
1982 & 0 & 2,870 & 0 & 0 & 2,870 \\
1983 & 0 & 131 & 0 & 0 & 131 \\
1984 & 0 & 1,220 & 221 & 0 & 1,441 \\
1985 & 0 & 632 & 893 & 0 & 1,525 \\
1986 & 0 & 2,016 & 2,636 & 0 & 4,652 \\
1987 & 0 & 2,700 & 1,447 & 0 & 4,147 \\
1988 & 0 & 131 & & & \\
& 0 & 2,870 & 2,636 & 0 & 131 \\
Minimum: & 342 & 1,434 & 746 & 0 & 4,652 \\
Maximum: & 43 & & & & 2,223 \\
Average: & & & &
\end{tabular}

\section{Spokane County Sawmill Residues 8}

\begin{tabular}{lrr}
\hline Year & $\begin{array}{c}\text { Total Residue } \\
\text { (Tons, dry weight) }\end{array}$ & $\begin{array}{r}\text { Total Used } \\
\text { (Tons, dry weigh }\end{array}$ \\
\hline $1988^{1}$ & & \\
$1986^{2}$ & 234,707 & 234,707 \\
$1984^{3}$ & 419,066 & 418,412 \\
$1982^{3}$ & 96,012 & 96,012 \\
$1980^{3}$ & 42,846 & 35,624 \\
$1978^{4}$ & 60,308 & 50,577 \\
& 195,820 & 123,178
\end{tabular}

$\begin{array}{ll}1 & \text { Includes Ferry and Pend Oreille counties } \\ 2 & \text { Includes Stevens county } \\ 3 & \text { Includes Pend Oreille county } \\ 4 & \text { Includes Pend Orelle and Stevens counties }\end{array}$

\section{Spokane County Secondary Wood Processing Facilities:}

SNE Enterprises, Inc.

East End, Bldg 14 Spokane Ind. Park

Spokane, WA 99216

Contact: Ken Kaiyala, Ray Willman

(509) $927-8300$

Residue (tons): 89

Jeld-Wen of Washington

P.O. Box 4364

Spokane, WA 99202

Contact: Frank DeVaul

(509) 534-0471

Residue (tons): 9,672
Design Millwork, Inc.

North 3808 Sullivan Road

Spokane, WA 99216

Contact: Dave Benghammer

(509) 924-0300

Residue (tons): 7,680

Eagle Woodworking

Route 4, Box 184

Spokane, WA 99204

Contact: Barnett \& Kent

(50̄9) 244-2058

Residue (tons): 3 
Interior Specialties

N 9527 Bruce Road

Spokane, WA 99207

Contact: Don Kassa

(509) 467-7418

Residue (tons): 4.32

Palanio Cabinets

P.O. Box 972

Deer Park, WA 99006

(509) 276-5436

Residue (tons): 0

T.R.A. Industries inc./Huntwood Industries Spokane Industrial Park, Bldg 26

Spokane, WA 99216

Contact: Tim Hunt

(509) 924-5858

Residue (tons): 51.975

Creative Wood Design

14511 East 18th Street

Veradale, WA 99037

Contact: Warren Smith

(509) 924-2787

Residue (Ibs): 200

D-Mac Carpentry

North 4828 Stevens

Spokane, WA 99205

Residue (Ibs): 260

R \& D Commercial Woodworking

W 1224 Grace

Spokane, WA 99205

(509) 448-4531

Residue (Ibs): 500

Wood Magic

E 1104 41st Avenue

Spokane, WA 99203

Contact: Victor \& Judy Schaum

(509) 624-9037

Residue (tons): 3.541
Columbia Cabinets, Inc.

West 1927 Maxwell

Spokane, WA 99201

(509) $328-2450$

Residue (tons): 0

Specialty Woods

16911 W Four Mound Road

Nine Mile Falls, WA 99026

Contact: Neal D. Bums

(509) 466-4684

Residue (lbs): 100

Kelley's Custom Cabinets

Route 2, Box 320-B

Deer Park, WA 99006

(509) 276-2015

Residue (Ibs): 1,200

Rick Ward Custom Cabinets

E 1606 Holyoke, \#4

Spokane, WA 99207

(509) 487-0048

Residue (tons): 1.20

Keller Cabinetry

N 22324 Orchard Bluff

Chattaroy, WA 99003

Contact: Leland A. Keller

Residue (Ibs): 438

Cooper's Cabinets, Inc.

East 3310 Sprague

Spokane, WA 99202

Contact: Steve Cooper

(509) 535-5539

Residue (Ibs): 600

Journeyman Cabinets, Inc.

E 2929 Providence

Spokane, WA 99207

Contact: Paul Ruckhaber

(509) 483-6864

Residue (tons): 4 
The Freight Crate

P.O. Box 15124

Spokane, WA 99215

(509) 928-3544

Residue (tons): 0

Pre-Built Structures, Inc.

$5315 \mathrm{~N}$ Corrigan

Otis Orchards, WA 99027

(509) 928-1442

Residue (tons): 3.41

\section{CURRENT USERS}

\section{Solid Fuel Processing Facilities:}

Spokane Pres-To-Logs

Spokane, WA

Plant Capacity (tons/yr):

Ave. Plant Output (tons/yr):

Gross Energy Equivalent (MBtu/yr):
Porta-Panel Buildings

13900 East Mission

Spokane, WA 99216

Residue (Ibs): 200
69,000

52,000

884,000 


\section{POTENTIAL USERS}

\section{Wood Combustion Facilties:}

W.I. Forest Products

Spokane, WA

Source: In-house

Biomass wood use bdt/yr: 19,764

Biomass energy output $\mathrm{mb}: 36$

Energy equiv mbtu/yr: 338,436

\section{Businesses:}

Camation Company

Spokane, WA 99220

Billion Btu Fuels: NA

Central Premix Concrete

Spokane, WA 99220

Billion Btu Fuels: 72.70

Consolidated Dairy Products

Spokane, WA 99208

Billion Btu Fuels: 30.24

Dellen Wood Products

Spokane, WA 99216

Billion Btu Fuels: 25.29

Ideal Cement

Spokane, WA 99212

Billion Btu Fuels: NA

Inland Empire Paper Co.

Spokane, WA 99212

No. of Boilers: 2

Boiler Fuels: NG

Billion Btu Fuels: 242.25

ITT Continental Baking Co.

Spokane, WA 99210

Billion Btu Fuels: 55.37
Layrite Products Co. Spokane, WA 99202

Billion Btu Fuels: 23.51

Olympic Foods, Inc Spokane, WA 99220

Billion Btu Fuels: 20.78

Quarry Tile Co.

Spokane, WA 99216

Billion Btu Fuels: 25.72

Ralston Purina Co.

Spokane, WA 99211

No. of Boilers: 2

Boiler Fuels: NG

Billion Btu Fuels: 42.84

Spokane Pres-To-Log Co.

Spokane, WA 99220

No. of Boilers: 2

Boiler Fuels: Wood/NG

Boiler Info Source: WSU

Billion Btu Fuels: 31.08

Spokane Seed Co.

Spokane, WA 99211

Billion Btu Fuels: 41.06

Standard Traffic Controls Spokane, WA 99211

Billion Btu Fuels: NA 
Kaiser Aluminum \& Chemical

Spokane, WA 99215

Boiler Fuels: NG

Billion Btu Fuels: 1477.80

\section{Colleges:}

Community Colleges of Spokane

N. 2000 Green Street

Spokane, WA 99207

(509) 536-7247

Spokane Community College

Sq.Ft. 684,858

Elect MMBtu: 33,009

Elect Cost: $\$ 413,712.50$

Fuel MMBtu: 56,130

Fuel Cost: $\$ 201,568.90$

Spokane Falls Community College

Sq.Ft. 407,012

Eloct MMBtu: 20,536

Elect Cost: $\$ 294,491.80$

Fuel MMBtu: 27,740

Fuel Cost: $\$ 98,311.95$

\section{Correctional Institutions:}

Pine Lodge Correction Center

P.O. Box C MS:

Medical Lake, WA 99022

(509) 299-5135, (Scan) 545-2902

Developmentally Disabled Centers:

Interlake School

Sq.Ft. 182,408

Elect MMBtu: 10,002

Elect Cost: $\$ 138,689.30$

Fuel MMBtu: 31,510

Fuel Cost: $\$ 95,357.88$
Westem Bottling Co., Inc.

Spokane, WA 99202

Billion Btu Fuels: 19.19

\author{
Eastern Washington University \\ Cheney, WA 99004 \\ (509) 359-6200 \\ Sq.Ft. 2,017,730 \\ Elect MMBtu: 72,546 \\ Elect Cost: $\$ 739,335.60$ \\ Fuel MMBtu: 188,103 \\ Fuel Cost: $\$ 569,421.80$
}

Eastern Washington University - Spokane

Center

Sq.Ft. 60,099

Elect MMBtu: 4,050

Elect Cost: $\$ 60,949.61$

Fuel MMBtu: 2,509

Fuel Cost: $\$ 9,578.79$
Lakeland Village

Sq.Ft. 490,624

Elect MMBtu: 21,019

Elect Cost: $\$ 292,312.80$

Fuel MMBtu: 100,035

Fuel Cost: $\$ 323,010.30$ 
Hospltals:9

Deaconess Medical Center

Type: Acute Care

Corporation Type: Non-Profit Corporation

Owner Name: Deaconess Medical Center, Inc.

Administrator: Thomas J. Zellers

Total Beds: 388

800 West Fitth Ave.

Spokane, WA 99210

(509) 458-5800

Deer Park Health Center and Hospital

Type: Acute Care

Corporation Type: Porprietary Corporation

Owner Name: Rural Health Services, Inc.

Administrator: Robert D. Brown

Total Beds: 26

Forest and " $D$ " Street

Dear Park, WA 99006

(509) 276-5061

Eastem State Hospital

Sq.Ft. 559, 176

Elect MMBtu: 22,609

Elect Cost: $\$ 312,287.80$

Fuel MMBtu: 117,541

Fuel Cost: $\$ 339,354.20$

Holy Family Hospital

Type: Acute Care

Corporation Type: Dominican Health Services

Owner Name: Holy Family Hospital

Administrator: Ronald Schurra

Total Bods: 272

North 5633 Lidgerwood

Spokane, WA 99207

(509) 482-0111

Mountainview Hospital

Type: Alcoholism

Corporation Type: Porprietary Corporation

Owner Name: Samissa Spokane, Inc.

Administrator: Ben Camp

Total Beds: 34

628 South Cowley

Spokane, WA 99202

(509) 624-3226
Sacred Heart Medical Center

Type: Acute Care

Corporation Type: Church

Owner Name: Sisters of Providence

Administrator: Gerald Leahy

Total Beds: 615

West 101 Eighth Avenue

Spokane, WA 99204

(509) 455-3131

Shriners Hospitals for Crippled Children

Type: Acute Care

Corporation Type: Non-Profit Corporation

Owner Name: Shriners Hospital for Crippled

Children, A Colorado Corp.

Administrator: Howard L. Parrett

Total Beds: 30

North 820 Summit Boulevard

Spokane, WA 99201

(509) 327-9521

St. Luke's Memorial Hospital

Type: Acute Care

Corporation Type: Non-Profit Corporation

Owner Name: St. Luke's Memorial Hospital

Administrator: Thomas M. White

Total Beds: 146

P.O. Box 288

South 711 COWLEY

Spokane, WA 99210

(509) 838-4771

Valley Hospital and Medical Center

Type: Acute Care

Corporation Type: Non-Profit

Owner Name: St. Luke's Memorial Hospital

Administrator: Michael Liepman

Total Beds: 123

Street 12606 East Mission Avenue

Spokane, WA 99216

(509) 924-6650 
Schools: ${ }^{10}$

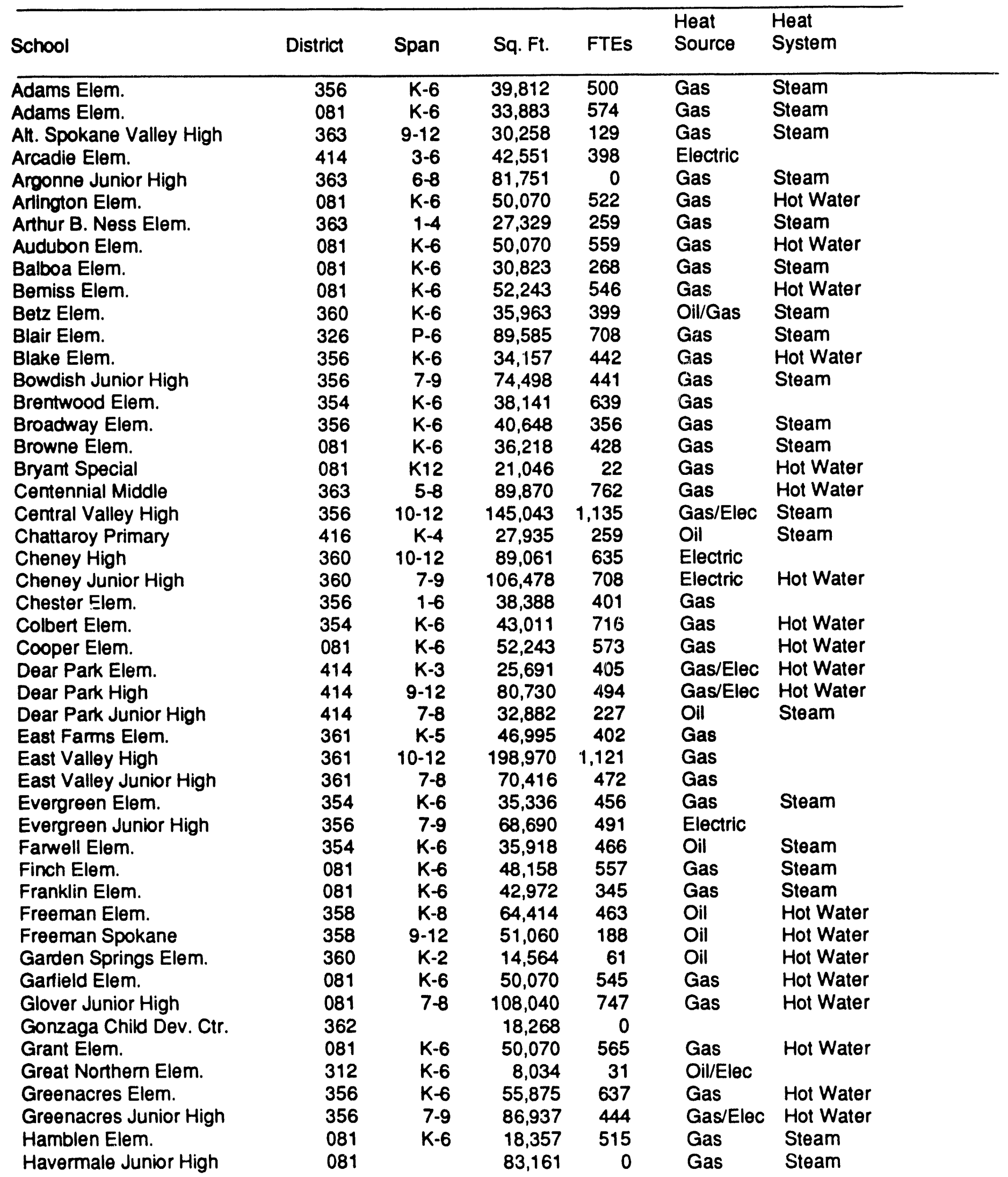


Schools: (continued)

\begin{tabular}{|c|c|c|c|c|c|c|}
\hline School & District & Span & Sq. Ft. & FTEs & $\begin{array}{l}\text { Heat } \\
\text { Source }\end{array}$ & $\begin{array}{l}\text { Heat } \\
\text { System }\end{array}$ \\
\hline Holmes Elem. & 081 & $\mathrm{~K}-6$ & 52,243 & 593 & Gas & Hot Water \\
\hline $\begin{array}{l}\text { Horizon Junior High } \\
\text { Hutton Elem. }\end{array}$ & 356 & $7-9$ & 84,795 & 490 & Gas & Hot Water \\
\hline Hutton Elem. & 081 & $\mathrm{~K}-6$ & 40,796 & 525 & Oil & Steam \\
\hline Indian Trall Elem. & 081 & $K-6$ & 26,416 & 357 & Gas & Hot Water \\
\hline Jefferson Elem. & 081 & $\mathrm{k}-6$ & 41,285 & 508 & Gas/Oil & Steam \\
\hline Joel E. Ferris High & 081 & $9-12$ & 213,250 & 1,525 & Gas & Hot Water \\
\hline John R. Rogers High & 081 & $9-12$ & 167,097 & 1,450 & Gas & Sieam \\
\hline Joseph Jantsch High & 081 & $9-12$ & 78,524 & 200 & Gas & Steam \\
\hline Keystone Elem. & 356 & P-6 & 33,669 & 305 & Gas & Hot Water \\
\hline Lake Spokane Elem. & 325 & $K-5$ & 17,670 & 493 & Electric & \\
\hline Lewis and Clark High & 081 & $9-12$ & 241,827 & 1,459 & Gas & Steam \\
\hline Libby Junior High & 081 & $7-8$ & 65,875 & 486 & Gas & Steam \\
\hline Liberty High & 362 & $9-12$ & 41,580 & 175 & Gas & Hot Water \\
\hline Liberty Junior High \& Elem. & 362 & $\mathrm{~K}-8$ & 47,500 & 388 & & \\
\hline Lincoln Heights Elem. & 081 & $\mathrm{~K}-6$ & 18,231 & 316 & OivGas & Steam \\
\hline Linwood Elem. & 081 & $k-6$ & 40,017 & 430 & Gas & Steam \\
\hline Logan Elem. & 081 & $\mathrm{~K}-6$ & 52,118 & 416 & Gas & Steam \\
\hline Longfellow Elem. & 081 & $\mathrm{~K}-6$ & 50,070 & 603 & Gas & Hot Water \\
\hline Madison Elem. & 081 & K-6 & 36,738 & 332 & Gas & Steam \\
\hline McDonald Elem. & 356 & $K-6$ & 30,949 & 308 & Gas & Steam \\
\hline Mead Junior High & 354 & $7-9$ & 109,841 & 855 & Gas & Steam \\
\hline Mead Senior High & 354 & $10-12$ & 212,985 & 1,726 & Gas & \\
\hline Medical Lake Elem. & 326 & K-6 & 44,261 & 557 & Gas/Elec & Steam \\
\hline Medical Lake High & 326 & $9-12$ & 81,524 & 433 & Gas & Steam \\
\hline Medical Lake Middle & 326 & $7-8$ & 52,519 & 253 & Gas & \\
\hline Midway Elem. & 354 & $k-6$ & 51,066 & 673 & Gas & Hot Water \\
\hline Milwood Early Childhood Ed. & 363 & P-1 & 27,132 & 215 & Gas & Steam \\
\hline Mountain View Jr. High & 361 & $6-8$ & 66,986 & 425 & Gas & \\
\hline Mullan Road Elem. & 081 & $\mathrm{~K}-6$ & 49,528 & 572 & Gas & Hot Water Steam \\
\hline New Jr./Sr. High Lakeside & 325 & & 84,555 & 0 & & \\
\hline Nine Mile Falls Elem. & 325 & $6-9$ & 30,377 & 395 & OillElec & \\
\hline North Central High & 081 & $9-12$ & 179,095 & 1,394 & Gas & Hot Water \\
\hline North Pines Junior High & 356 & $7-9$ & 102,170 & 394 & Gas & Steam \\
\hline Northwood Junior High & 354 & $7-9$ & 100,829 & 660 & Gas & Steam \\
\hline Opportunity Elem. & 356 & $\mathrm{k}-6$ & 42,848 & 459 & Gas & Hot Water \\
\hline Orchard Center Elem. & 363 & $1-4$ & 28,984 & 272 & Gas & Steam \\
\hline Orchard Prairie Elem. & 123 & $\mathrm{~K}-7$ & 7,309 & 52 & Gas & \\
\hline Otis Orchards Elem. & 361 & $K-5$ & 35,104 & 503 & Oil & \\
\hline Pasadena Park Elem. & 363 & $1-4$ & 27,615 & 279 & Gas & Steam \\
\hline Ponderosa Elem. & 356 & $K-6$ & 51,377 & 426 & Gas & Hot Water \\
\hline Pratt Elem. & 081 & $\mathrm{~K}-6$ & 21,424 & 329 & Gas & Steam \\
\hline Progress Elem. & 356 & $1-3$ & 37,573 & 201 & Gas & Steam \\
\hline Regal Elem. & 081 & $k-6$ & 52,243 & 543 & Gas & Hot Water \\
\hline Ridgeview Elem. & 081 & $\mathrm{~K}-6$ & 23,291 & 438 & Oilvas & Steam \\
\hline Riverside High & 416 & $9-12$ & 48,272 & 507 & & \\
\hline Riverside Middle & 416 & $5-8$ & 60,056 & 570 & Electric & \\
\hline Riverside Primary & 416 & $P-4$ & 52,984 & 456 & Electric & \\
\hline Roosevelt Elem. & 081 & $\mathrm{~K}-6$ & 50,070 & 599 & Gas & Hot Water \\
\hline
\end{tabular}


Schools: (continued)

\begin{tabular}{|c|c|c|c|c|c|c|}
\hline School & District & Span & Sq. Ft. & FTEs & $\begin{array}{l}\text { Heat } \\
\text { Source }\end{array}$ & $\begin{array}{l}\text { Heat } \\
\text { System }\end{array}$ \\
\hline Sacajawea Junior High & 081 & $7-8$ & 112,613 & 938 & Gas & Hot Water \\
\hline Salk Junior High & 081 & $7-8$ & 110,386 & 655 & Gas & Hot Water \\
\hline Salnave Elem. & 360 & K-6 & 49,737 & 406 & Electric & Hot Water \\
\hline Seth Woodard Primary & 363 & & 28,659 & 0 & Oil & Steam \\
\hline Shadle Park High & 081 & $9-12$ & 260,025 & 1,309 & Gas & Steam \\
\hline Shaw Junior High & 081 & $7-8$ & 112,613 & 551 & Gas & Hot Water \\
\hline Sheridan Elem. & 081 & $k-6$ & 50,070 & 512 & Gas & Hot Water \\
\hline Shiloh Hills Elem. & 354 & $K-6$ & 51,066 & 522 & Gas & Hot Water \\
\hline Skills Center & 081 & $11-12$ & 69,994 & 0 & Gas & Hot Water \\
\hline Skyview Elem. & 361 & $K-5$ & 42,090 & 342 & Gas & \\
\hline South Pines Elem. & 356 & $k-6$ & 41,640 & 437 & Gas & Hot Water \\
\hline Spokane Gamy Junior High & 081 & $7-8$ & 98,878 & 545 & Gas & Hot Water \\
\hline Spokane Guilds Room & 081 & & 1,717 & 0 & & \\
\hline Stevens Elem. & 081 & $K-6$ & 53,808 & 466 & Gas & Steam \\
\hline Sunrise Elem. & 356 & $K-6$ & 53,673 & 577 & Gas & Hot Water \\
\hline Sunset Elem. & 360 & $K-6$ & 45,769 & 322 & Electric & Hot Water \\
\hline Trent Elem. & 361 & K-5 & 58,171 & 323 & Gas & \\
\hline Trentwood Elem. & 361 & $K-5$ & $46, i 15$ & 393 & Gas & \\
\hline University Elem. & 356 & $K-6$ & 37,947 & 514 & Gas & Steam \\
\hline University High & 356 & $10-12$ & 144,522 & 1,032 & Gas/Oil & Steam \\
\hline West Valley High & 363 & $9-12$ & 117,892 & 739 & Gas & Steam \\
\hline Westview Elem. & 081 & $K-6$ & 39,068 & 480 & Gas & Steam \\
\hline Whitman Elem. & 081 & K-6 & 52,243 & 627 & Gas & Hot Water \\
\hline Whitworth Elem. & 354 & & 30,826 & 0 & Gas & Steam \\
\hline Willard Elem. & 081 & $\mathrm{~K}-6$ & 50,070 & 590 & Gas & Hot Water \\
\hline Wilson Elem. & 081 & $K-6$ & 28,099 & 369 & Gas/Elec & Steam \\
\hline Windsor Elem. & 360 & $K-6$ & 37,919 & 443 & Oil & Steam \\
\hline Woodridge Elem. & 081 & $K-6$ & 50,070 & 503 & Gas & Hot Water \\
\hline
\end{tabular}

State B:- Idings:

Cheney Cowles Museum

Sq.Ft. 48,950

Elect MMBtu: 1,759

Eloct Cost: $\$ 26,424.87$

Fuel MMBts 4,429

Fuel Cost: $\$ 16,179.63$

Department of Transportation - Dist. 6

Headquarters, Spokane

Sq.Ft. 63,690

Elect MMBtu: 2,928

Elect Cost: $\$ 49,579.93$

Fuel MMBtu: 7,913

Fuel Cost: $\$ 29,544.38$

Court of Appeals -- Spokane Ottice

Sq.Ft. 14,654

Elect MMBtu: 848

Elect Cost: $\$ 14,302.63$

Fuel MMBtu: 617

Fuet Cóst: \$2,5400.79

Spokane District 4 Headquarters

Sq.Ft. 17,467

Elect MMBtu: 792

Elect Cost: $\$ 14,574.59$

Fual AMABtu: 0

Fuel Cost: $\$ 0.00$ 


\section{Appendix B Sources}

1 Office of Financial Management. Data Book 1989. 1989

2 The Intormation Press. 1986 Washington State Yearbook. 1986

3 U.S. Department of Commerce. County Business Patterns 1986. 1986

4 Northwest Public Power Association. 1989 NW Electric utility Directory. 1989.

5 Washington State Department of Agriculture. Agricultural Statistics. 1982-1989.

6 Washington State Department of Ecology.

7 Washington State Department of Natural Resources. Timber Harvest Report. 1981-1988.

8 Washington State Department of Natural Resources. Mill Surveys. 1978-1988.

9 Washington State Department of Health. Directory of Licensed Hospitals. 1989.

10 Superintendent of Public Instruction 

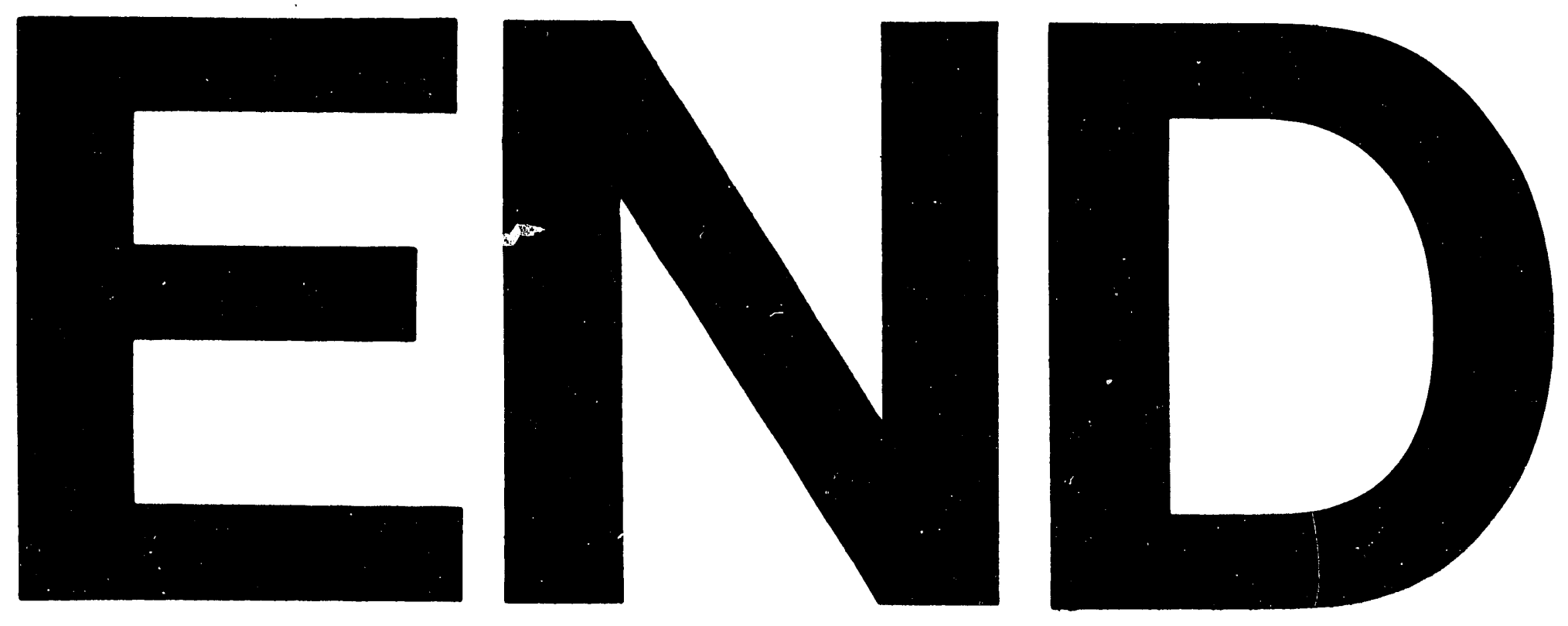

I

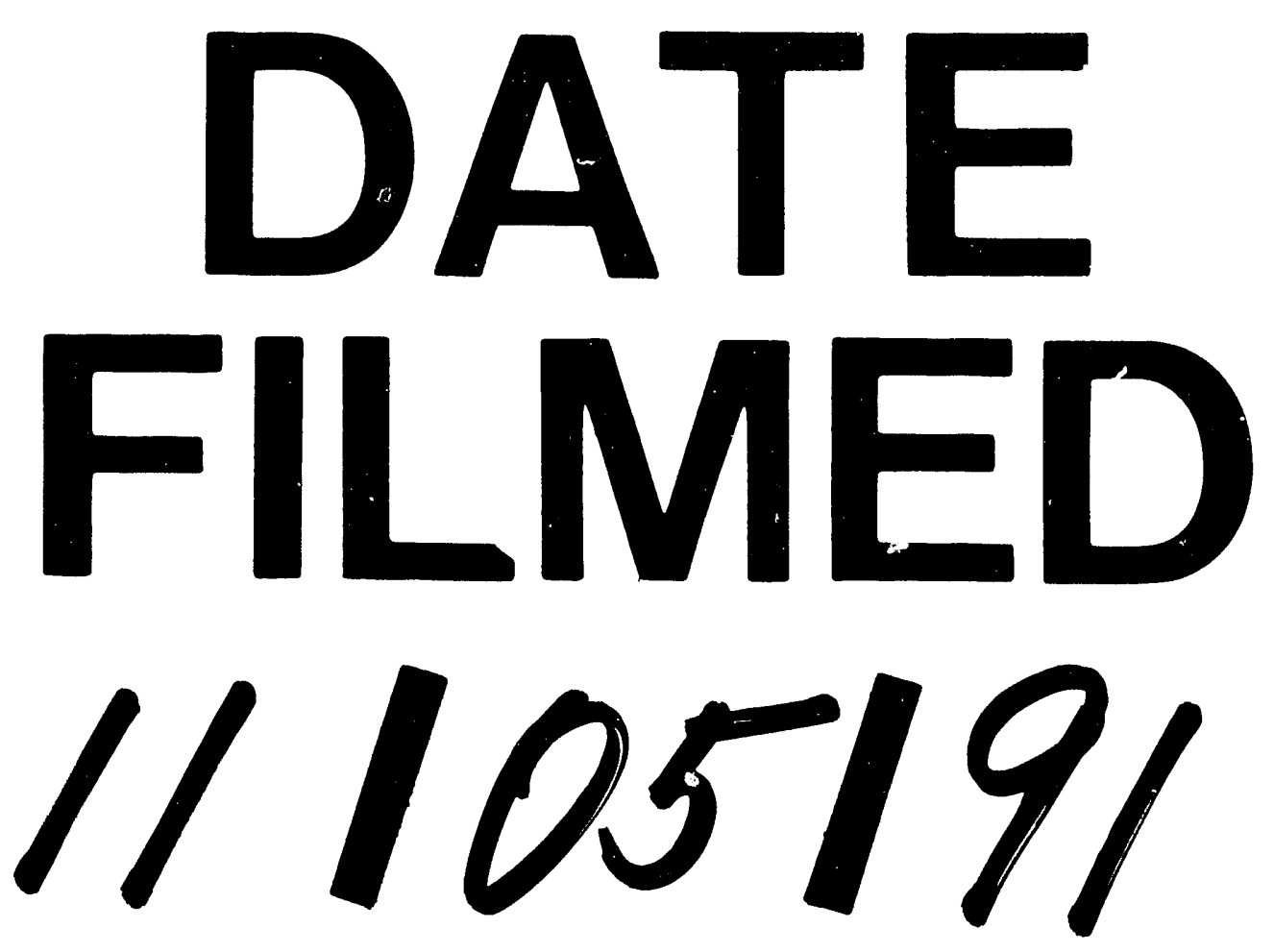


